\title{
Genetic and behavioral correlates of pair living in coppery titi monkeys (Plecturocebus cupreus)
}

\author{
Dissertation \\ for the award of the degree \\ "Doctor of Philosophy" Ph.D. Division of Mathematics and Natural Sciences \\ of the Georg-August-Universität Göttingen \\ within the doctoral program Biodiversity and Ecology \\ of the Georg-August University School of Science (GAUSS)
}

Submitted by

Sofya Dolotovskaya

From Ufa, Russia

Göttingen, 2020 


\section{Thesis Committee}

Prof. Dr. Eckhard W. Heymann, Department of Behavioral Ecology and Sociobiology, German Primate Center, Germany

PD Dr. Christian Roos, Primate Genetics Laboratory, German Primate Center, Germany PD Dr. Oliver Schülke, Department of Behavioral Ecology, Johann-Friedrich-BlumenbachInstitute for Zoology \& Anthropology, University of Göttingen, Germany

\section{Members of the Examination Board}

Reviewer: Prof. Dr. Eckhard W. Heymann, Department of Behavioral Ecology and Sociobiology, German Primate Center, Germany

Second reviewer: Prof. Dr. Julia Fischer, Cognitive Ethology Laboratory, German Primate Center, Germany

\section{Further members of the Examination Board}

PD Dr. Christian Roos, Primate Genetics Laboratory, German Primate Center, Germany PD Dr. Oliver Schülke, Department of Behavioral Ecology, Johann-Friedrich-BlumenbachInstitute for Zoology \& Anthropology, University of Göttingen, Germany Prof. Dr. Mark Maraun, Johann-Friedrich-Blumenbach-Institute for Zoology \& Anthropology, University of Göttingen, Germany Dr. Sven Bradler, Department of Animal Evolution and Biodiversity, Johann-FriedrichBlumenbach-Institute for Zoology \& Anthropology, University of Göttingen, Germany 


\section{CONTENTS}

SUMMARY

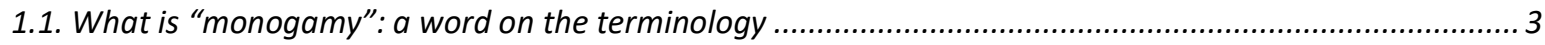

1.2. Why does "monogamy" occur: an overview of hypotheses and comparative studies................................ 4

1.3. Genetic monogamy: ultimate drivers and proximate mechanisms......................................................... 7

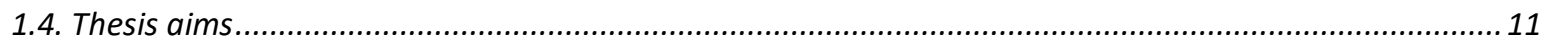

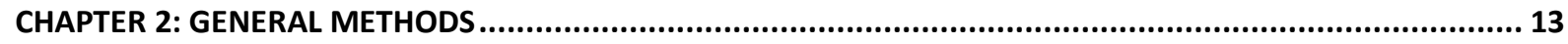

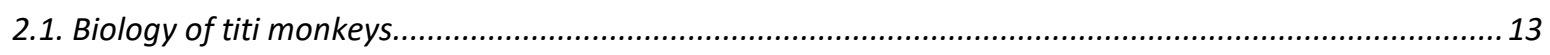

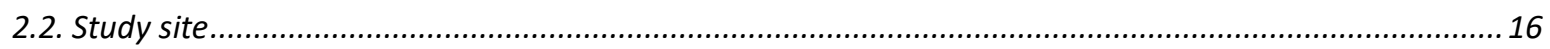

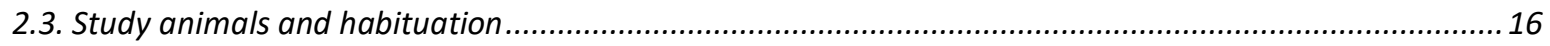

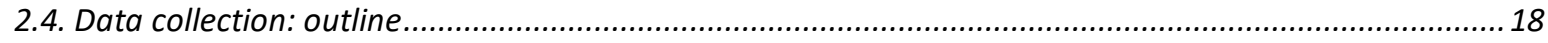

CHAPTER 3: GENETIC MONOGAMY AND MATE CHOICE IN A PAIR-LIVING PRIMATE ............................... 19

CHAPTER 4: WHAT MAKES A PAIR BOND IN A NEOTROPICAL PRIMATE: FEMALE AND MALE CONTRIBUTIONS

CHAPTER 5: DO LESS OR EAT MORE: STRATEGIES TO COPE WITH COSTS OF PARENTAL CARE IN A PAIR-LIVING

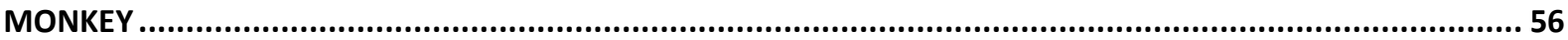

CHAPTER 6: ACTIVE ANTI-PREDATOR BEHAVIOUR IN RED TITI MONKEYS (Plecturocebus cupreus) ............... 72

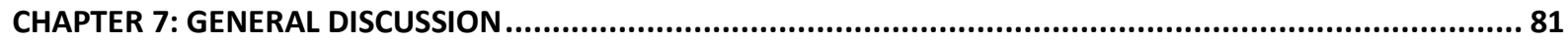

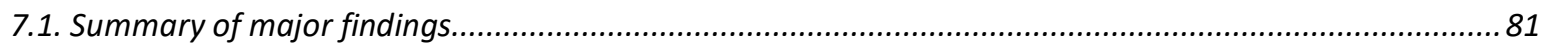

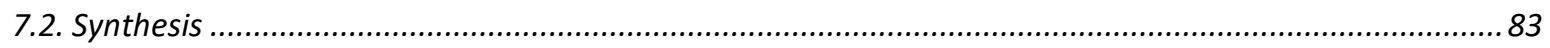

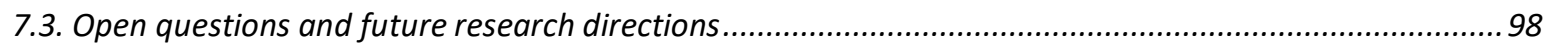

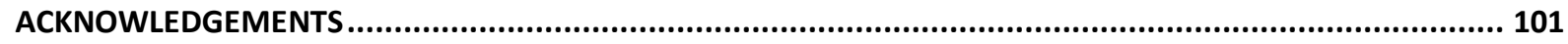

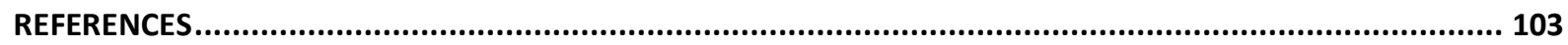

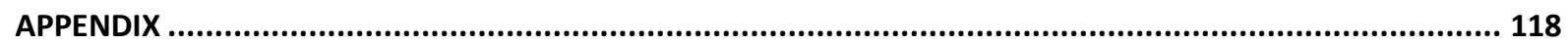

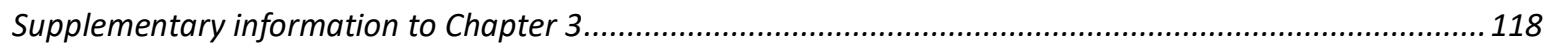

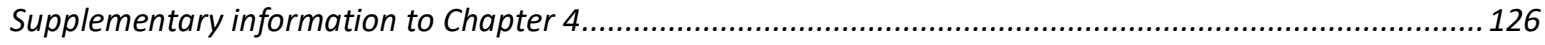

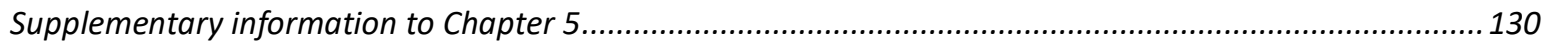

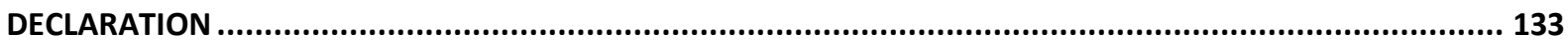




\section{SUMMARY}

The occurrence of monogamy in mammals represents an evolutionary puzzle. Because of reduced parental investment, males are expected to increase their reproductive success by mating with multiple females rather than being committed to one mate. Still, some mammals are socially monogamous, or pair living, and several species are even genetically monogamous. In some pair-living species, "monogamy package" further includes biparental care and pair bonding. To understand why monogamy occurs, it is necessary to examine the relationships between different elements of this package and the factors that influence them. Neotropical titi monkeys of genera Callicebus, Plecturocebus, and Cheracebus are not only socially monogamous, but also have a remarkably high level of male care, where infants are carried almost exclusively by males, and form strong pair bonds, a rare combination among mammals. The aim of this study was to investigate whether this combination is translated into genetic monogamy and which mechanisms help to maintain this social system.

To achieve this, I examined mating system and its genetic and behavioral correlates in a wild population of coppery titis, Plecturocebus cupreus, at the Estación Biológica Quebrada Blanco in Peruvian Amazon. I collected fecal samples for genetic analyses from 41 individuals of 14 family groups, including 18 offspring of nine family groups (up to five offspring generations per group). Seven of these groups were also subject to behavioral observations, during which I collected data on social interactions, activity budgets and territorial behaviors of adult males and females (total sampling time 2749 h, focal observation time 384 h, 14 months in total). I showed that coppery titis were mostly genetically monogamous, as paternity analyses based on 18 microsatellite loci found no cases of extra-pair paternity. As the costs of pairing with closely related or incompatible mate can be high in monogamous species, I further examined if mate choice was based on relatedness or heterozygosity. Mating was random with regard to relatedness and heterozygosity. Relatedness between mates in ten observed pairs did not differ from the average relatedness in randomly generated pairs, and heterozygosity of mates was not correlated. Despite the absence of evidence for active inbreeding avoidance via mate choice, pair mates were on average not related (mean $r=-0.033$ ). To see if this low relatedness could be explained by natal dispersal, I conducted spatial genetic analysis. No spatial genetic structure was found in either sex, indicating that dispersal was opportunistic, with both sexes migrating over varying distances. These findings suggest that even opportunistic dispersal, as long as it is unconstrained, can generate sufficient genetic diversity in the population to prevent inbreeding. As pair-living species are known to sometimes engage in extra-pair copulations to minimize inbreeding, the sufficient genetic diversity can help to maintain genetic monogamy by rendering these extra-pair copulations unnecessary. 
At our study site, unconstrained dispersal was likely facilitated by the relatively low population density in undisturbed habitat.

To investigate behavioral mechanisms of monogamy maintenance, I examined female and male contributions to the pair bond, territorial defense, and infant care. Females were found to contribute more than males to the maintenance of proximity and affiliation within pairs. They groomed males more than vice versa and made most of the approaches and leaves within pairs. Males, on the other hand, contributed more than females to the territorial defense, participating in the intergroup encounters more often and more actively. These findings are consistent with the concept of a pair bond as an exchange of services, where females contribute to proximity and affiliation maintenance in exchange for services provided by males. These services, in addition to the territorial defense, include intensive infant care and protection from predators. The analysis of activity budgets and diet composition in caring adults indicated that after infant birth, females foraged more and consumed more proteinrich food (arthropods) than before. This would probably not be possible if the females had to carry the infants themselves, because arthropod foraging is not well compatible with infant carrying. And indeed, males foraged less and consumed less arthropods when they were carrying infants. In line with these findings, grooming between pair mates was more heavily skewed toward female investment during the period of infant dependency, when male services are most needed. Finally, males provided protection from predators, as their antipredator behavior was more active than that of females. Altogether, these findings indicate that while females maintain the pair bond, males provide services important to females, namely infant care, protection from predators and territorial defense.

In conclusion, this study indicates that three factors are important for maintaining social and genetic monogamy in coppery titis: pair bond, male care and possibilities for unconstrained dispersal. Strong pair bond, supported by contributions of both pair mates, likely limits the opportunities for extra-pair copulations and thus maintains genetic monogamy. Hight level of male care helps to reinforce social and genetic monogamy by freeing the female from the costs of infant carrying. This allows the females to forage more to compensate for the energetic costs of lactation, presumably contributing to their increased fecundity, and also makes the males more attractive to the females. Finally, unconstrained dispersal likely helps to maintain both social and genetic monogamy by preventing deviations from pair-living and keeping the inbreeding at a low level. In sum, these findings demonstrate that not only the components of social system (pair bond and male care) but also habitat characteristics (possibility for unconstrained dispersal) can play important roles in maintaining pair living and promoting genetic monogamy in pair-living species. 


\section{CHAPTER 1: GENERAL INTRODUCTION}

\subsection{What is "monogamy": a word on the terminology}

"Monogamy" has long been of interest for biologists and anthropologists, and there is a considerable amount of research on it. However, what exactly is meant by "monogamy" in the literature is not always clear. Over the years, this term has been used by different authors to refer to pair living, pair bonding, sexual monogamy, genetic monogamy and even biparental care (Huck et al., 2020; Kleiman, 1977; Tecot et al., 2016). Historically, these were often considered as integral parts of one "monogamy package" (Fuentes 2000). Sexual monogamy, for example, was usually assumed to be an outcome or an obligate correlate of social monogamy. But as molecular tools became more accessible, it became clear that "monogamy" has several components that are not necessarily associated with each other. For example, over $90 \%$ of birds were originally considered as "monogamous" (Lack 1968). However, since the first application of DNA fingerprinting in 1987 that demonstrated extra-pair paternity (EPP) in the house sparrow, Passer domesticus (Burke \& Bruford 1987), EPP has been detected in 76\% of pair-living bird species with biparental care (Brouwer and Griffith, 2019). Even the white stork, Ciconia ciconia, traditionally considered a symbol of monogamy, was found not to be genetically monogamous (Turjeman et al. 2016).

Today, many combinations of "monogamy" components are known, and it has been shown that even sexual and genetic monogamy are not always associated with each other. Some animals, such as Azara's owl monkeys, Aotus azarae, exhibit the whole "monogamy package", including pair living, pair bonding, genetic monogamy and biparental care (Huck et al. 2014). Other species may lack one or more components. For example, Kirk's dik-diks, Madoqua kirkii, are pair living, pair bonded and genetically monogamous but do not have biparental care (Brotherton et al. 1997). Eurasian beavers, Castor fiber, live in pairs and have biparental care but the pairs are not cohesive and do not mate monogamously (Nimje et al. 2019; McClanahan et al. 2020). Fork-marked lemurs, Phaner furcifer, are pair living but do not exhibit any other "monogamy" traits (Schülke \& Kappeler 2003). Finally, Western jackdaws, Corvus monedula, are pair living and genetically monogamous, but the application of modern surveillance technology has demonstrated that they often engage in extra-pair copulations (Gill et al. 2019).

In a recent review on pair-living and sexual monogamy, Huck et al. (2020) argued that inconsistent use of monogamy-related terms has often led researchers to "compare apples with oranges". They proposed a new terminology clearly defining separate aspects of monogamy, based on the framework proposed by Kappeler and van Schaik (2002) for 
describing social systems and later corroborated by Kappeler (2019). In this thesis, I will follow this terminology, defined in Box 1.

\section{Box 1. Terminology used in this study}

Pair-living (who lives with whom): a type of social organization where one adult male and one adult female share a home range, possibly with their non-reproducing offspring. This is often referred to as "social monogamy".

Pair-bonded (who is affiliated with whom): a type of social structure where a female-male pair have an affiliative relationship to the exclusion of other adults, as evidenced by behavioral, emotional, and/ endocrinological characteristics.

Sexual monogamy (who mates with whom): a type of social mating system where one adult male and one adult female have an exclusive mating relationship during at least one reproductive season.

Genetic monogamy (who produces offspring with whom): a type of genetic mating system where one adult male and one adult female produce offspring exclusively with each other over a set of multiple births (at least one reproductive season for species that produce more than one infant per litter and over more than one consecutive reproductive seasons for species with singleton births)

Biparental care (who provides parental care): a type of care system where a mother and putative father regularly provide offspring care.

\subsection{Why does "monogamy" occur: an overview of hypotheses and comparative studies}

The occurrence of pair living, or social monogamy, in animals represents an evolutionary puzzle. As a result of reduced investment in gametes and parental care, males have higher potential reproductive success than females (Trivers 1972; Bateman 1948; Clutton-Brock \& Parker 1992). It is therefore unclear why a male should be committed to only one female if he could instead increase his reproductive success by mating with multiple females. This paradox is especially pronounced in mammals as they have internal fertilization, gestation, and lactation (Trivers 1972). And indeed, social monogamy is rare in mammals, in contrast to birds where it is more prevalent (Griffith et al. 2002; Lack 1968). ${ }^{1}$

\footnotetext{
1 Estimations of the number of pair-living mammals vary depending on the classification method used: $3 \%$ (Kleiman, 1977), 9\% (Lukas and Clutton-Brock, 2013).
} 
Early hypotheses aimed to explain the evolution of monogamy largely distinguished between two types of it, obligate and facultative (Kleiman 1977). Obligate monogamy was considered to evolve in response to a need for male care (Wittenberger \& Tilson 1980; Clutton-Brock 1989). And facultative monogamy was thought to evolve when female distribution in space does not allow male to monopolize more than one female at a time (Emlen \& Oring 1977). However, this obligate-facultative dichotomy was eventually abandoned, because comparative analyses showed that paternal care does not always correlate with the occurrence of obligate monogamy in primates (Wright 1990; Komers \& Brotherton 1997; Tardif 1994). Also, it became clear that in some species without biparental care, for example, in Kirk's dik-diks, monogamy is not exactly facultative because males do not attempt to monopolize more than one female, even when they are available (Brotherton et al. 1997; Komers \& Brotherton 1997).

The more recent hypotheses try to distinguish between the different components of "monogamy" (reviewed in, e.g., Fernandez-Duque and Fiore, 2020; Huck et al., 2020; Klug, 2018; and Tecot et al., 2016). According to the "infant care" hypothesis, pair living and pair bonding may evolve when the mother needs help from others to successfully raise the offspring; consequently, a male benefits from staying with one female and helping her with the offspring (Wittenberger \& Tilson 1980; Kleiman 1977). The "infanticide prevention" hypothesis, proposed originally for primates, states that pair living and its behavioral correlates evolve when a male helps a female to protect offspring from infanticidal males (van Schaik \& Dunbar 1990; van Schaik \& Kappeler 2003). The "female dispersion" hypothesis posits that that female over-dispersion in space, caused by resource distribution, does not allow males to monopolize access to more than one female, leading to social and/or sexual monogamy (Emlen \& Oring 1977). Similar to it is the "mate guarding" hypothesis that states that states that it is more beneficial for males to guard one female than to seek additional females; unlike the "female dispersion" hypothesis, it predicts that males will not attempt to mate with more females even if they can (Emlen \& Oring 1977; Brotherton \& Komers 2003). Finally, according to the "resource defense" hypothesis, pair living may be the most stable strategy for joint resource defense, while a more general case of this idea, the "optimal group size" hypothesis, states that the trade-offs between food competition and protection from predators may lead to an optimal group size of only two breeding adults (Terborgh \& Janson 1986).

Over the past decades, these hypothesis were tested in a number of comparative analyses, many of them conducted on primates because pair living is widespread in them in comparison to other mammals (about one-fifth of species: Kappeler and Pozzi, 2019). Female space use 
was indicated as the most likely ultimate driver of social and sexual monogamy in several studies. An early phylogenetic analysis on mammals suggested that sexual monogamy likely evolved when females were solitary and occupied small, exclusive ranges, enabling males to guard and monopolize them (Komers \& Brotherton 1997). These conclusion were later supported by a large-scale comparative phylogenetic study based on a dataset of ca. 2500 mammalian species; it indicated that sexual monogamy likely evolved where breeding females are intolerant of each other and their density was low, and males were therefore unable to defend access to multiple females (Lukas \& Clutton-Brock 2013) ${ }^{2}$. A recent comparative phylogenetic study on primates also supported the role of female space use in the evolution of pair living, indicating that it has most often evolved from solitary ancestors and served as a stepping stone toward the evolution of group living (Kappeler \& Pozzi 2019). Support for this "increasing complexity" model of social evolution has been found also in Artiodactyla, birds and insects (Jaeggi et al. 2020; Cornwallis et al. 2010; Hughes et al. 2008). Male care has been shown to commonly co-occur with social and sexual monogamy and to evolve more easily in pair-living lineages (Lukas \& Clutton-Brock 2013; Opie et al. 2013). However, comparative analyses in mammals indicate that it was most likely a consequence rather than a cause of social and/or sexual monogamy, facilitated by the proximity between a male and offspring (Lukas \& Clutton-Brock 2013; Komers \& Brotherton 1997; Opie et al. 2013). Nevertheless, it appears to be an important proximate mechanism of the maintenance of social and sexual monogamy. By relieving a female from some of the energetic costs of offspring care, male contribution to care may allow a female to regain body condition quickly after weaning and mate sooner, invest more time in foraging and/or produce more or better quality milk, all of this contributing to offspring well-being and increased reproductive success of both sexes (Woodroffe \& Vincent 1994). A recent comparative phylogenetic analysis on mammals has demonstrated that male care indeed benefits both sexes through increased female fecundity, mediated by a reduction in lactation time (West \& Capellini 2016). Higher female fecundity, in turn, makes up for the energetic and opportunity costs of caring for the males, reducing the costs of social and sexual monogamy. As a result, a positive evolutionary feedback between the increased female fecundity and male care may appear, reinforcing the maintenance of monogamy (West \& Capellini 2016).

The role of infanticide risk in the evolution of social monogamy has not received much support in comparative analyses. So far, only one study, conducted on primates, indicated that social

\footnotetext{
2 In their paper, Lukas and Clutton-Brock (2013) discuss "social monogamy". However, as Huck et al. (2020) have shown in their detailed review, Lukas and Clutton-Brock (2013) actually used the breeding status of female in their classification of mammalian species, and what they called "social monogamy" appeared to be sexual monogamy.
} 
monogamy is best explained by infanticide risk (Opie et al. 2013). However, recent studies suggest that reduction of infanticide risk was a consequence rather than a driver of evolutionary transitions to pair living (Lukas \& Huchard 2014; Kappeler 2014; Kappeler \& Pozzi 2019).

Finally, comparative studies on primates have demonstrated that phylogenetic history is an important factor explaining the occurrence of social and sexual monogamy (Shultz et al. 2011; Kappeler \& Pozzi 2019; Lukas \& Clutton-Brock 2013). The social organization, social structure and mating systems of Old World monkeys, as well as social organization of Eulemur species (a diverse radiation of lemurs), are highly uniform, despite the great ecological variability in these lineages (Di Fiore \& Rendall 1994; Ossi \& Kamilar 2006). Social structure was also better explained by phylogeny than by environment in macaques (Thierry et al. 2000). Overall, social system components were shown to be relatively conserved at lower taxonomic levels, with the closely related species generally having the same social or mating system (Shultz et al. 2011; Kappeler \& Pozzi 2019).

\subsection{Genetic monogamy: ultimate drivers and proximate mechanisms}

Since social organization of animals is often a poor indicator of their mating system, it is important to study the genetic consequences of mating and the relationships between social and mating systems to better understand their evolution (Huck et al. 2014). Because sexual selection in both males and females is influenced by the number of mating partners and the reproductive success, the important role in the evolution of mating systems is played by EPP (Petrie \& Kempenaers 1998; Clutton-Brock 2007). The levels of EPP vary greatly between different species, and even within species, mating systems have often been found to be dynamic, with individuals adopting different mating strategies under different ecological conditions (e.g., Brouwer and Griffith, 2019; Kitchen et al., 2006; Wright et al., 2010). Examining why some individuals, populations or species have higher EPP rates than others is therefore crucial for our understanding of the evolution of mating systems.

Many comparative studies on the EPP variation have been conducted on birds because pair living is common among them, and most of the species have at least some level of EPP (Brouwer \& Griffith 2019; Griffith et al. 2002). However, despite the extensive amount of data collected over the years, it still remains largely unclear why some pair-living bird species have higher EPP rates than others. A recent review of all paternity studies in birds conducted in the last 30 years concluded that there is no universal explanation for EPP variation. Rather, different behaviors and ecological variables were shown to affect EPP in different species or populations of the same species (Brouwer \& Griffith 2019). In mammals, the data are much 
scarcer than in birds, as the number of pair-living species is overall lower, and for many of them, genetic paternity data are still not available. In the only comparative phylogenetic study on pair-living mammals conducted to date, ${ }^{3}$ EPP rates across 15 species were associated with the intensity of male care and, weakly, with the strength of pair bond (Huck et al. 2014).

As in the case of social and sexual monogamy, the variation in EPP rates have a very strong phylogenetic signal. In birds, 39-50\% of the interspecific variation in EPP levels occurs between families and orders, rather than among closely related species (Griffith et al. 2002; Brouwer \& Griffith 2019). This suggests that many interspecific differences of the EPP rates are more likely to be explained by evolutionary history than by contemporary factors. In line with this, an hierarchical explanation for variation in EPP has been proposed, with EPP rates at different organizational levels determined by different ecological, genetic and social correlates (Griffith et al. 2002; Arnold \& Owens 2002). The convenience of the hierarchical explanation is illustrated, for example, by a study of species of the Maluridae family (fairywrens and relatives), where different factors explained variation in EPP rates at different levels of organization (Brouwer et al. 2017).

When trying to understand the reasons for the variation in EPP rates at the lower levels of populations and individuals, an interesting question is which behaviors or local ecological and demographic factors affect the probability of extra-pair copulations (EPC). ${ }^{4}$ One of the behaviors shown to affect the probability of EPC and, consequently, EPP is mate guarding. Mate guarding is often indirectly assessed by the strength of the pair bond, where mates with close bonds are guarded more closely and thus have less opportunities for EPC. The strength of pair bond, measured as the spatial cohesiveness of pair mates and used as a proxy for mate guarding, was associated with EPP levels at the population level in the meta-analysis in birds (Harts et al. 2016). Even at the interspecific level, mammal species with stronger bonds were shown to have lower EPP rates (Huck et al. 2014). In addition to pair bond strength, mate guarding can be affected by other factors, such as male age or habitat structure (Nimje et al. 2019; Ramos et al. 2014).

The probability of EPC is also affected by the availability of potential mates, which, in turn, can be influenced by population density and breeding synchrony (Westneat 1990). Higher

\footnotetext{
${ }^{3}$ Studies of Cohas and Allainé (2009) and Lambert et al. (2018) are not discussed, as they included group-living mammals with a dominant breeding pair and cooperative breeders in the category of socially monogamous mammals.

${ }^{4}$ It is important to note that EPC do not necessarily translate to EPP, as copulations might not end in fertilizations as a result of postcopulatory selection mechanisms such as sperm competition or cryptic female choice (Griffith 2007; Griffith et al. 2002). For example, in Western jackdaws, Corvus monedula, despite frequent EPC, no evidence for EPP was found, likely because of the frequent intrapair copulations and mate guarding (Gill et al. 2019). By contrast, in superb fairywren, Malurus cyaneus, $76 \%$ of young are sired by extrapair males, and yet EPC has never been directly observed in $>10$ years of research (Double \& Cockburn 2000).
} 
population density is expected to make encounters between individuals more likely, and positive relationship between EPP rates and population density was demonstrated in several comparative studies on birds, both within and across populations (e.g., Brouwer et al., 2017; Westneat and Sherman, 1997). Breeding synchrony was suggested to affect EPP rates in two different directions. On one hand, EPP rates can be lower in animals with high breeding synchrony as a result of a trade-off between searching for EPC and parental care (Birkhead \& Biggins 1987). On the other hand, synchronous breeding can allow female to simultaneously compare different males, thus increasing EPP rates (Stutchbury \& Morton 1995). The evidence for the link between EPP and breeding synchrony remains mixed (Brouwer \& Griffith 2019; Isvaran \& Clutton-Brock 2007).

Finally, a big question is why some individuals engage in EPC while others do not - in other words, how do individuals make their mating decisions, or mate choice? Whether an individual chooses to restrict matings to its social partner or to seek EPC, fitness benefits of their mate choice can be generally divided into direct and indirect (genetic) benefits (reviewed in Andersson and Simmons, 2006). Direct benefits are resources, such as food, territory, parental care, and they are usually more likely to be offered by intra-pair mates. Indirect, or genetic fitness benefits can be gained both in intra- and extra-pair matings and are expressed in the offspring heterozygosity and, consequently, their fitness (heteorozygosity and genetic compatibility hypotheses: Brown, 1997; Zeh and Zeh, 1996, 1997; reviewed in Kempenaers, 2007). Therefore, to gain indirect benefits, animals are expected to choose mates that are unrelated or dissimilar at some fitness-related genes (e.g., genes of the major histocompatibility complex, MHC). In addition, individuals can choose heterozygous mates to gain direct benefits, because heterozygous partners are expected to have higher fitness and should be more likely to provide increased parental care, fertility or good quality territory (Kempenaers 2007; Hoffman et al. 2007). Mate choice based in relatedness, variation at the MHC genes or heterozygosity has been demonstrated in various species of mammals and birds (e.g., García-Navas, Ortego, \& Sanz, 2009; Hoffman et al., 2007). However, for many pair-living species, the evidence for mate choice based on relatedness or genetic similarity remains absent or mixed (e.g., Hansson et al., 2007; Huchard, Knapp, Wang, Raymond, \& Cowlishaw, 2010; Sommer, 2005).

The limitations of mate choice in the pair-living animals have been suggested as one of the reasons why individuals might seek EPC. In pair-living animals, especially in those with biparental care, mate choice can be severely constrained. Not only mates become unavailable once paired, but also individuals might face a trade-off between choice for direct and indirect benefits. As a result, individuals may end up paired to genetically incompatible or closely 
related partner. To escape these constraints, animals might engage in EPC that would allow them to gain indirect benefits while still taking advantage of direct benefits provided by the social partner (Jennions \& Petrie 2000; Brooker et al. 1990). And indeed, numerous studies in birds found a positive relationship between relatedness or genetic similarity of social partners and the EPP rates, as well as differences in the heterozygosity levels between withinpair and extra-pair offspring (Griffith 2010; Arct et al. 2015). In mammals, however, the evidence for relationship between EPP and relatedness or genetic similarity of social partners is very limited. To our knowledge, the only pair-living mammal for which this effect has been demonstrated to date is fat-tailed dwarf lemurs, Cheirogaleus medius, where females sharing more MHC-supertypes with their social partner engaged in more EPC (Schwensow et al. 2008).

One of the biggest problems arising from the constraints of mate choice in pair-living animals is the risk of inbreeding. In the absence of other options, or as a result of a conflict between settling in a territory and avoiding inbreeding, individuals might pair with too closely related mates. This problem can be solved "actively" by either avoiding matings with closely related individuals (through kin recognition) or engaging in EPC with less related individuals, as discussed above (Leedale et al. 2020; Jennions \& Petrie 2000). Alternatively, "passive" inbreeding avoidance can be ensured by natal dispersal that disrupts opposite-sex kin associations and thus allows to avoid matings between them (Greenwood 1980). However, it is not clear if dispersal has to be sex-biased to generate enough local genetic dissimilarity between breeding females and males to avoid inbreeding. In mammals, dispersal is most often male-biased as a consequence of stronger male intra-sexual competition in polygynous species, such as most mammals (Greenwood 1980; Dobson 1982). But it is still not well understood whether dispersal in pair-living mammals is sex-biased, too, and the evidence remains mixed, with some species showing similar rates of dispersal for females and males and others demonstrating female-biased dispersal (Mayer et al. 2017b; Fernandez-Duque 2009; Ribble 1992; Favre et al. 1997). Moreover, many open questions remain about the relationships between dispersal, inbreeding avoidance and mating patterns.

To sum up, current evidence suggests that there is no single ultimate driver of pair-living or genetic monogamy across animals. However, many factors have been identified that affect the evolution and maintenance of social and genetic monogamy, among them female use of space, male care, pair bond, mate guarding, mate choice and population density and dynamics, influenced, in turn, by resource use and distribution. All these factors can interact with each other, and the relative importance of each of them likely differs across different levels of organization. To better understand how social and genetic monogamy is maintained and 
might have evolved, it is important to examine not only the influence of each of these factors on monogamy, but also the relationships between them at different organizational levels, such as individuals, groups, populations and species. In birds, there is extensive research on these topics. In mammals, however, only a handful of studies have addressed both genetic and behavioral aspects of monogamy, partly because monogamy is much rarer in mammals than it is in birds.

\subsection{Thesis aims}

Neotropical titi monkeys of genera Callicebus, Plecturocebus, and Cheracebus are unusual among mammals in exhibiting almost all the elements of the "monogamy package". They are pair living, with groups comprising one reproductive pair and one to three offspring, and form long-term pair bonds with high level of spatial cohesiveness between mate mates (Van Belle, Fernandez-Duque, et al. 2016; Anzenberger 1988; Fernandez-Duque et al. 2013; BiccaMarques \& Heymann 2013; see Chapter 2 for more details on titi biology). Titis have an exceptionally high level of male care, where the infant is carried almost exclusively by the male from the first week of life and is returned to the mother only to suckle (Wright 1984; Spence-Aizenberg et al. 2016; Bicca-Marques \& Heymann 2013). The only missing monogamy component which has yet to be characterized is the genetic mating system. Although titis represent a textbook example of a "monogamous" primate, their mating system has not been examined in any of the species yet. Likewise, almost nothing is known about the proximate influences on titis' mating system, such as mate choice, dispersal patterns or kinship structure of their populations. Finally, little data is available on behavioral mechanisms that maintain pair living in titis, such as pair bond maintenance and territorial defense.

The overall aim of this study was to examine genetic and behavioral correlates of pair living in coppery titi monkeys, Plecturocebus cupreus. First, I examined if pair living translated into genetic monogamy and analyzed the mechanisms of genetic monogamy maintenance in Chapter 3. Second, I addressed two behavioral correlates of pair living, pair bonding and biparental care, in Chapters 4 and 5. To further address the mechanisms of pair-living and pair-bonding maintenance, I examined the sex differences in anti-predator behaviors in Chapter 6. To achieve these aims, I studied a wild population of coppery titis in Peruvian Amazon using a combination of observational and genetic methods. This is the first comprehensive study addressing both behavioral and genetic correlates of pair living in a wild population of titis. The structure of this study is depicted in Fig. 1. 
Specifically, I addressed the following questions:

1. Does pair living in coppery titis translate into genetic monogamy (Chapter 3):

(a) what is the genetic mating system of titis?

(b) is mate choice in titis based on relatedness or heterozygosity?

(c) how do titis avoid inbreeding?

(d) is dispersal sex-biased in titis?

2. What are the mechanisms of pair-bond maintenance in coppery titis (Chapter 4):

(a) what are the contributions of each sex to the pair-bond maintenance?

(b) which factors affect the strength of the pair bond?

3. How do coppery titis deal with the costs of biparental care (Chapter 5):

(a) how does infant care affect the pair bond?

(b) how do activity patterns change in lactating females and infant-carrying males?

(c) how does diet composition change in lactating females and infant-carrying males?

4. What are the anti-predator behaviors in coppery titis (Chapter 6):

(a) does one sex participate in anti-predator defense more actively than another?

(b) do titis use active anti-predator behaviors?

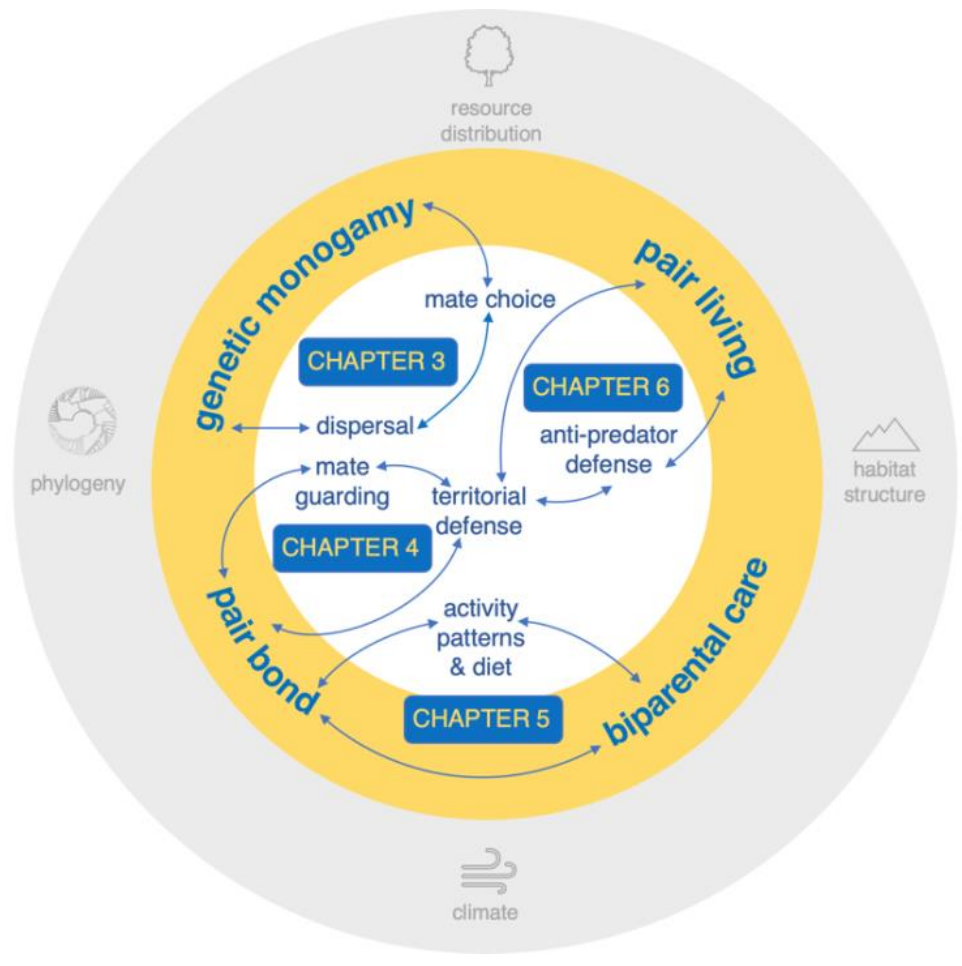

Fig. 1. Relationships between the four elements of "monogamy" and their proximate mechanisms examined in this study. Not all possible interactions and proximate influences are included. 


\section{CHAPTER 2: GENERAL METHODS}

\subsection{Biology of titi monkeys}

The coppery titi monkey, Plecturocebus cupreus, belongs to a diverse radiation of Neotropical primates of the family Pitheciidae (titis, sakis, and uacaris). Titis, comprising up to 35 species in the most recent classifications (Boubli et al. 2019; Byrne et al. 2016; Câmara Gusmão et al. 2019), inhabit an extensive range from the foothills of the northern Andes throughout the rainforests of Amazon and upper Orinoco basins, the gallery forests of the Llanos region of Colombia, dry forests and Chaco of Paraguay and Bolivia, to the Atlantic forests and wooded savanna of Cerrado region of Brazil. Traditionally, all titi species were assigned to one genus, Callicebus Thomas 1993, but a recent taxonomic revision supported the division of titi monkeys into three genera: Plecturocebus, Cheracebus and Callicebus (Byrne et al. 2016). Cheracebus species are sympatric with Plecturocebus species in western Amazon basin, while the Atlantic Callicebus species are separated from the other two genera by the Cerrado and Caatinga of central Brazil. Phylogenetic analyses and fossil record indicate that Cheracebus was the oldest radiation within titis, diverged ca. 11 million years ago, while Callicebus and Plecturocebus diverged 9-8 million years ago (Byrne et al. 2016; Perelman et al. 2011).

Titis are small- to medium-sized arboreal monkeys, with adult body mass of $0.8-1.7 \mathrm{~kg}$ (Smith \& Jungers 1997; Heymann et al. 2012; Defler 2004; Norconk 2020). They have non-prehensile tails and little or no physical dimorphism between the sexes (sexual dimorphism ratio in body mass 0.85-1.16: Heymann et al., 2012; Norconk, 2011; Smith and Jungers, 1997). Titis feed mainly on fruits supplemented by invertebrates, leaves and flowers, with the proportions of these components in the diet varying between species (Defler 2004; van Roosmalen et al. 2002; Byrne et al. 2016; Bicca-Marques \& Heymann 2013). They can be preyed upon by capuchin monkeys, carnivores, raptors and snakes (see Chapter 6 for the full list of predators).

Titis live in family groups typically comprising one reproductive pair and up to three young (Van Belle, Fernandez-Duque, et al. 2016; Defler 2004; Norconk 2011; Bicca-Marques \& Heymann 2013). Although there are a few reports of titi groups with extra adult-sized individuals, likely resulting from delayed offspring dispersal, most studies indicate that titis almost exclusively live in groups with only one adult individual of each sex (Felton et al. 2006; Cäsar et al. 2012; Van Belle, Fernandez-Duque, et al. 2016; Bicca-Marques et al. 2002; Price \& Piedade 2001; Bicca-Marques \& Heymann 2013). Family groups range over an area of 5-10 ha on average and up to 50 ha in rare cases, although in highly fragmented forests home range size may be less than 1 ha (Norconk 2020; Bicca-Marques \& Heymann 2013).

The relationships between group members are generally very peaceful, and intra-group aggression in wild titis is extremely rare (some examples: Lawrence, 2007; Spence-Aizenberg 
et al., 2016). All group members frequently groom each other and rest in body contact, especially pair mates, who spend most of the time within few meters of each other (almost $50 \%$ of time within $1 \mathrm{~m}$ in this study) and engage in long grooming sessions that can for last several hours and account for 5-10\% of daily activity time (Fernandez-Duque et al., 2013; Kinzey and Wright, 1982; Lawrence, 2007; Spence-Aizenberg et al., 2016; Chapter 4). Perhaps the most characteristic affiliative behavior in titis is tail-twining when two or more animals interlace their tails while sitting side by side; this behavior gave name to the genus Plecturocebus, meaning "tail-twining long-tailed monkey" in Greek). Another prominent behavior is duetting, a coordinated loud calling that is performed by pair mates and is often joined by the offspring to form a chorus. Duets and choruses are most often performed in the morning and during intergroup encounters and are thought to play a role in resource and mate defense (Caselli et al. 2014; Robinson 1981; Kinzey \& Robinson 1983; Robinson 1979). Titis have a remarkably high level of male care. Both in the wild and in captivity, the infant is carried almost exclusively by the adult male from the first week of life and is returned to the mother only to suckle; males also play with offspring and share food with them more often than females (Wright 1984; Spence-Aizenberg et al. 2016; Tirado Herrera \& Heymann 2004; Lawrence 2007; Jantschke et al. 1995; Fragaszy et al. 1982; Bicca-Marques \& Heymann 2013). Titis typically give birth to a single infant once a year, with most births occurring between September and January in wild groups and year-round in captivity (Valeggia et al. 1999; Van Belle, Fernandez-Duque, et al. 2016; Souza-Alves et al. 2019; Bicca-Marques \& Heymann 2013). The interbirth intervals are close to one year both in the wild and in captivity and are shorter after an early death of the infant (during the first year) than after the infant's survival (11.9 vs. 14.2 months in wild P. discolor), indicating the absence of seasonal ovarian inactivity (Van Belle, Fernandez-Duque, et al. 2016; Jantschke et al. 1995; Valeggia et al. 1999). Twinning in wild titis has been reported only three times, in P. cupreus, $P$. toppini and $C$. coimbrai (Knogge \& Heymann 1995; Lawrence 2007; de Santana et al. 2014). Females have no external signs of ovulation, and cycle length in captivity average 17 days (Valeggia et al., 1999). Gestation lasts ca. 4.5 months, followed by ca. 6.5 months of lactational anovulation (data from captivity: Jantschke et al., 1995; Valeggia et al., 1999).

Offspring disperse from their natal groups at the age of 2-4 years, presumably after reaching sexual maturity, although no data on age at first reproduction are available for wild titis (Van Belle, Fernandez-Duque, et al. 2016; Palacios et al. 1997; Bicca-Marques \& Heymann 2013). In captivity, females gave birth to their first infant when they are on average 3.7 years old (Valeggia et al. 1999), and in the wild, a female was observed to copulate at the age of 2.6. years, after dispersing from her natal group at the age of 2.2 years (Van Belle, Fernandez- 
Duque, et al. 2016). Both sexes seem to disperse, although it is unknown how far each sex migrates (Palacios et al. 1997; Van Belle, Fernandez-Duque, et al. 2016; Bossuyt 2002).

Generally, very little is known about demography or life history of wild titis. The scarcity of data can be partly attributed to the difficulty of studying wild pitheciids in general and titis in particular. As noted in the recent book on pitheciids, "titis are almost pathologically shy, and seem to spend most of their time moving quietly in dense vegetation" (Veiga et al. 2013). To date, the longest dataset available for titis is a 12-year study on red titis, Plecturocebus discolor (previously Callicebus discolor) (Van Belle et al., 2016), and there are no published studies on genetic mating system or kinship structure on any wild titi population.

Coppery titis, P. cupreus (previously Callicebus cupreus), are found in the tropical rainforests of the western Amazonia: south of Rio Napo and Rio Solimões and to the headwaters of the Rios Juruá and Purús in Brazil, from the east bank of Rio Ucayali to the west bank of Rio Purús (van Roosmalen et al. 2002; Hershkovitz 1990) (Fig. 2). Coppery titis have a body mass of ca. 750-1200 g, with males being slightly larger than females(Heymann et al. 2012). The only field site for which data on wild P. cupreus is available is Estación Biológica Quebrada Blanco $(\mathrm{EBQB})^{1}$, where this study has been conducted. Two groups at EBQB have been followed intermittently since 1997, and accounts of their activity budgets, diet composition and ranging patterns have been published (Kulp \& Heymann 2015; Nadjafzadeh \& Heymann 2008). There are, however, almost no data on demography and life history of $P$. cupreus (only occasional birth records at EBQB are available: Knogge and Heymann, 1995; Terrones Ruiz et al., 2004; Tirado Herrera and Heymann, 2004).

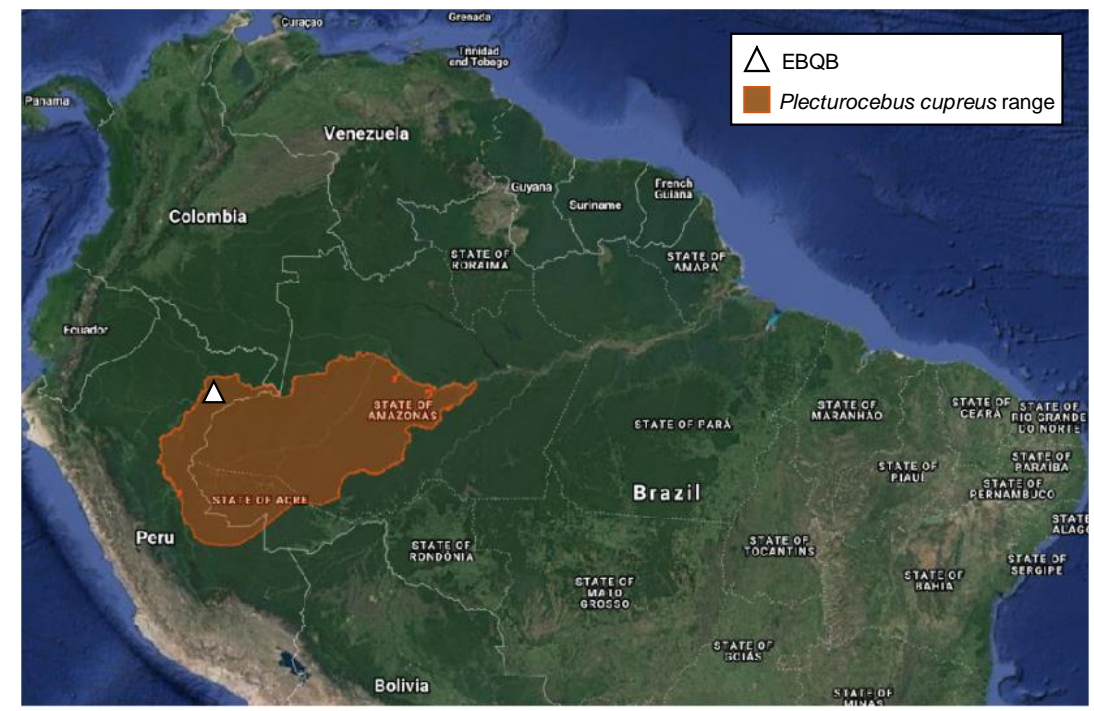

Fig. 2. The distribution of Plecturocebus cupreus and the location of the Estación Biológica Quebrada Blanco (EBQB). The range map is obtained from www.alltheworldsprimates.org/Home.aspx.

\footnotetext{
${ }^{1}$ A population in Northwestern Brazil, studied by Bicca-Marques et al. (2002), although reported as $P$. cupreus (Callicebus cupreus under the old classification), is located to the east of Rio Purús and should be classified as $P$. toppini (Byrne et al. 2016).
} 


\subsection{Study site}

This study was conducted in June 2017-December 2018 at the Estación Biológica Quebrada Blanco (EBQB) in the north-eastern Peruvian Amazonia (4²1'S $73^{\circ} 09^{\prime} \mathrm{W}$, ca. $120 \mathrm{~m}$ above sea level) (Fig. 2). The study area consists mainly of primary tropical rainforest of the "terra firme" type (not inundated during the rainy season) interspersed with small swampy areas. The home ranges of three study groups (Groups 2, 3, and 13, see Fig. 4 below) also included land strips along the river (Quebrada Blanco) that can be inundated for a few days during the height of the rainy season. Rainfall shows a strongly seasonal pattern, with a dry season $(<250$ $\mathrm{mm} / \mathrm{month}$ ) between June and November and a wet season ( $\geq 250 \mathrm{~mm} / \mathrm{month}$ ) between December and May (data for 1997-2016: Heymann et al., 2019). In 2017 and 2018, the rainfall generally followed the same pattern, with June-September being relatively dry $(\leq 200$ mm/month; Fig. 3). However, in February 2017 and 2018 (the middle of the wet season) and in November 2018 (transition from dry to wet season), rainfall dropped below 200 $\mathrm{mm} /$ month. This likely reflects the trend towards more erratic rainfall fluctuations in the western Amazon basin during the recent years, reported by both local people and long-term studies (e.g., Haghtalab et al., 2020, showed a higher number of extreme droughts and floods since 1982). Total rainfall in 2017 and 2018 was $2947 \mathrm{~mm}$ and $2719 \mathrm{~mm}$, respectively.

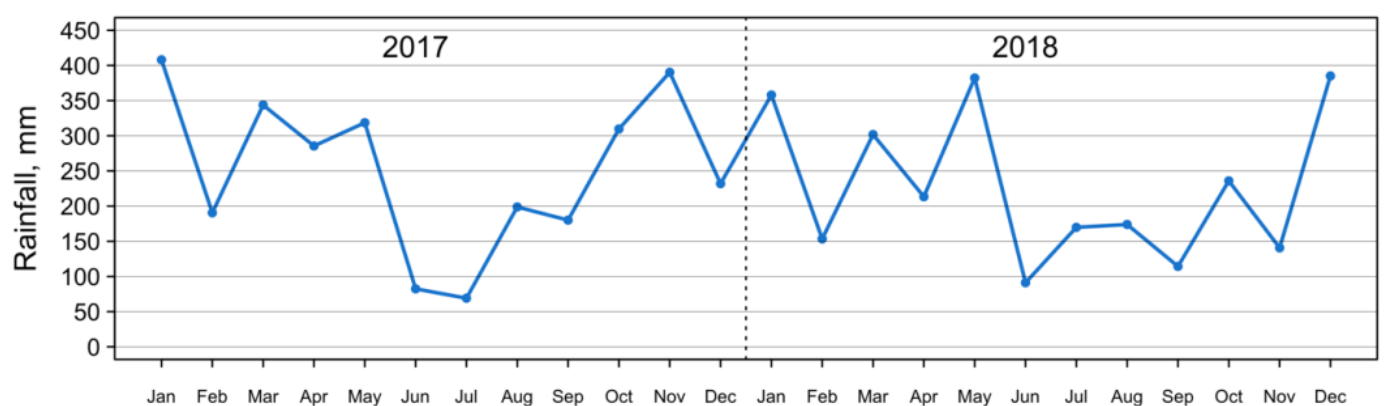

Fig. 3. Monthly variation of rainfall at Tamshiyacu $\left(4^{\circ} 00^{\prime} 10.7^{\prime \prime} \mathrm{S} 73^{\circ} 09^{\prime} 38.2^{\prime \prime} \mathrm{W}\right)$, ca. $40 \mathrm{~km}$ north of EBQB, in 2017 and 2018. Raw data downloaded from Servicio Nacional de Meteorología e Hidrología del Perú (www.senamhi.gob.pe).

\subsection{Study animals and habituation}

Study individuals belonged to 14 family groups (Fig. 4). Group 1 had been habituated to the presence of human observers and studied intermittently since 1997; the other groups were habituated during this study by teams of 2-3 local field assistants. Details on study groups are provided in Chapter 3. We considered a group fully habituated when the animals allowed us to approach to within $5 \mathrm{~m}$ without fleeing or hiding. On average, it took 6 (3-10) weeks to habituate a group. Some groups, however, could never be habituated. For example, the animals from Group 12, after being followed intermittently for almost 8 months, still ran from the observers when they tried to approach them, and eventually we had to abandon the group. 
Interestingly, the group is known for more than 15 years, but could never be fully habituated in the past either. Another group that had to be abandoned after more than 10 weeks of unsuccessful attempts was Group 14.

Home range sizes varied from 3.6 to 12.98 ha (mean = 7.2 ha; Chapter 3 ) and overlapped only slightly between neighbouring groups (mean $=1.4 \%$, varying from 0 to $4.7 \%$; Chapter 3, Fig. 4). The exception is the home range of Group 11 that was newly established following the dispersal of a subadult male from his natal Group 1 and was most likely not permanent and bound to shift later (more details on the dispersal event in Chapter 3).

Seven of the groups (1-7) were subject to both behavioral (Chapters 4-6) and genetic (Chapter 3) studies; the remaining groups were used only to collect samples for genetic analyses (Chapter 3). We individually identified all the study animals based on the combination of body size, tail shape and colouration, and genital size and shape.

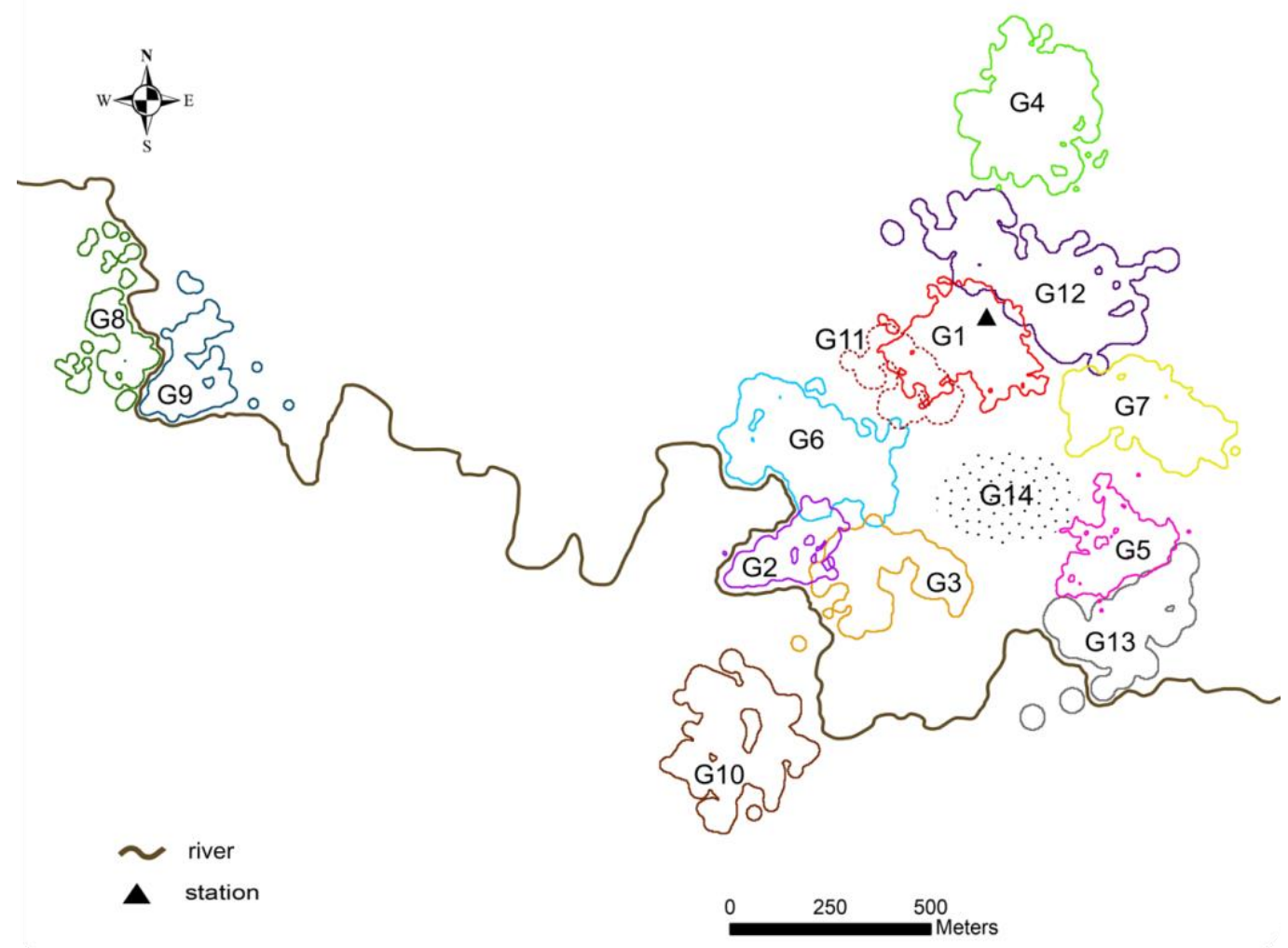

Fig. 4. Home ranges of study groups, estimated using the $95 \%$ fixed kernel density method with ArcGIS Desktop 10.6 (ESRI). The home range of Group 14 is depicted as dotted ellipse because we did not have enough GPS data to reliably estimate its home range. The home range of Group 11 is depicted as dotted line because this newly established territory was most likely not permanent and bound to shift later (see Chapter 3 for details). ${ }^{2}$

\footnotetext{
${ }^{2}$ The spatial gap between Groups 8-9 and the rest of the groups is a result of study logistics, not the lack of titis in that area. Most of the groups were located around the camp because they were easier to access and the animals were already partly habituated to human presence; Groups 8-9, located near the house of one of the local field assistants, were chosen for the same reason. The gap between Groups 2, 3 and 6 on one hand and of Groups 1, 5 and 7 on the other hand is partly occupied by a secondary forest (abandoned buffalo pasture regenerating since 2000) that seems to be avoided by titis (Kulp \& Heymann 2015). During the study period, we never saw titis inside this area; Group 14 occupies the primary forest.
} 


\subsection{Data collection: outline}

The study was conducted in June 2017-December 2018. Behavioral data were collected during two field seasons, June-December 2017 and June-December 2018. Each group was followed in blocks of 5-6 days by a team of two people from the early morning when the animals left a sleeping site (or from when the groups was located) until the late afternoon when the animals retired to a sleeping site (or until the group was lost). The main focus of behavioral data collection was the social interactions of breeding adults, infant care behavior, and territorial behavior, described in Chapters 4 and 5 . These data were collected by me throughout both field seasons and additionally by two volunteer field assistants in 2018 . We used continuous focal animal sampling of the adult male and female of each group; the details of sampling method are provided in Chapters 4 and 5 . The second person following the group (local field assistant) recorded the GPS position of the groups every 10 min using Garmin GPS Map 62/64s and used scan sampling to record identity and activity of each visible individual. Additionally, any significant behaviors that did not occur frequently enough to be recorded during focal animal or instantaneous scan sampling (copulations, predator encounters and attacks, duetting, intergroup encounters, predator, etc.) were opportunistically recorded. Outside of the two main field seasons, study groups were monitored for 1-2 days per month by the local field assistants for the changes in group composition. Genetic samples were collected continuously by me and all the field assistants from the beginning of the study until September 2019. The details of genetic data collection are provided in Chapter 3. 


\section{CHAPTER 3: GENETIC MONOGAMY AND MATE CHOICE IN A PAIR-LIVING PRIMATE}

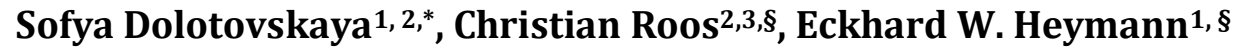

\footnotetext{
${ }^{1}$ Behavioral Ecology and Sociobiology Unit, German Primate Center, Göttingen, Germany

2 Primate Genetics Laboratory, German Primate Center, Göttingen, Germany

${ }^{3}$ Gene Bank of Primates, German Primate Center, Göttingen, Germany

$\S \mathrm{CR}$ and EWH share senior authorship
}

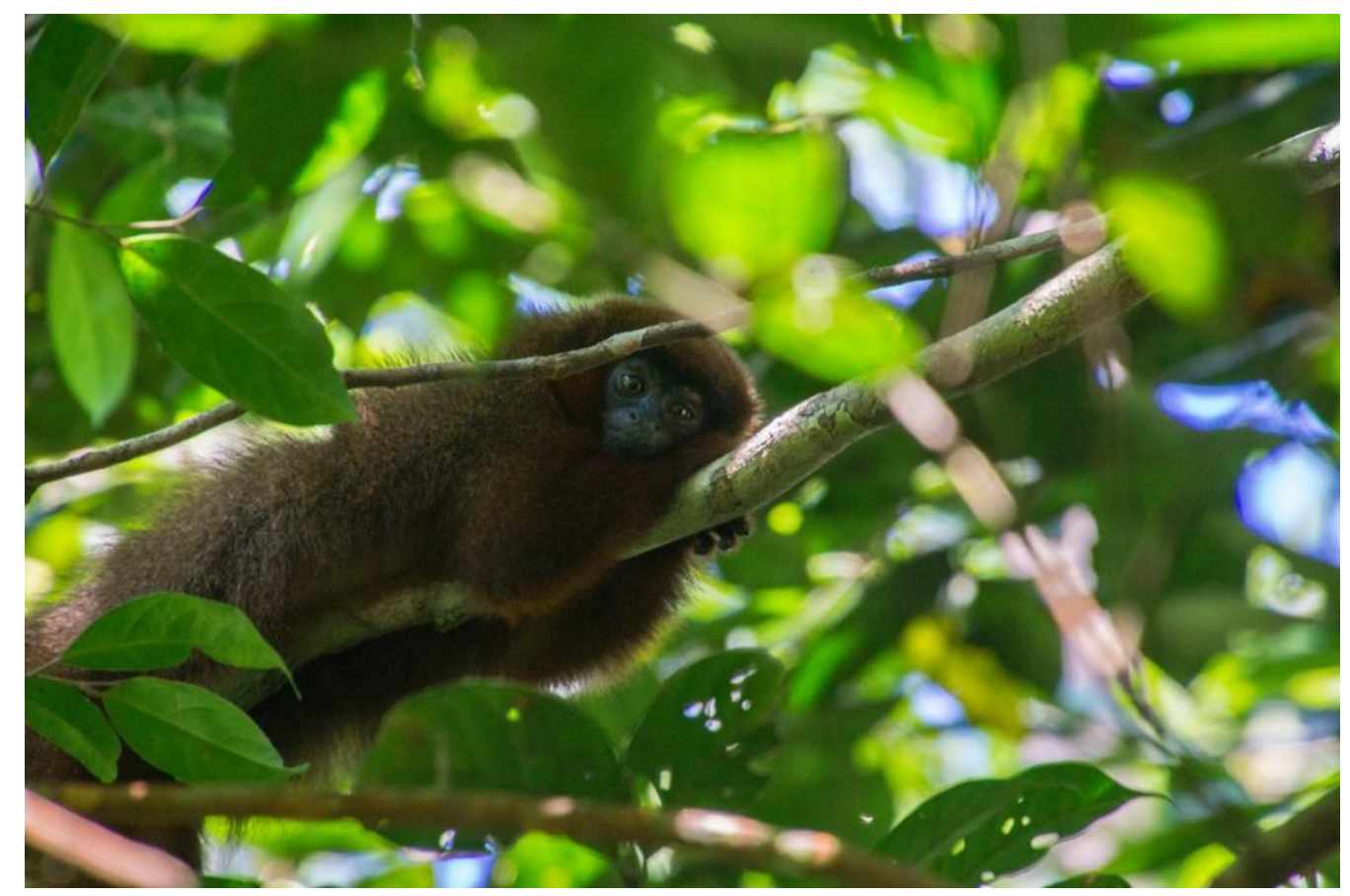

2020

Scientific Reports

https://doi.org/10.1038/s41598-020-77132-9

(Accepted: 2 November 2020) 


\section{Abstract}

In pair-living mammals, genetic monogamy is extremely rare. One possible reason is that in socially monogamous animals, mate choice can be severely constrained, increasing the risk of inbreeding or pairing with an incompatible or low-quality partner. To escape these constraints, individuals might engage in extra-pair copulations. Alternatively, inbreeding can be avoided by dispersal. However, little is known about the interactions between mating system, mate choice and dispersal in pair-living mammals. Here we genotyped 41 wild individuals from 14 groups of coppery titi monkeys (Plecturocebus cupreus) in Peruvian Amazon using 18 microsatellite loci. Parentage analyses of 18 young revealed no cases of extra-pair paternity, indicating that the study population is mostly genetically monogamous. We did not find evidence for relatedness- or heterozygosity-based mate choice. Despite the lack of evidence for active inbreeding avoidance via mate choice, mating partners were on average not related. We further found that dispersal was not sex-biased, with both sexes dispersing opportunistically over varying distances. Our findings suggest that even opportunistic dispersal, as long as it is not constrained, can generate sufficient genetic diversity to prevent inbreeding. This, in turn, can render active inbreeding avoidance via mate choice and extra-pair copulations less necessary, helping to maintain genetic monogamy.

\section{Introduction}

Since sexual selection in both males and females is influenced by the number of mating partners, extra-pair paternities (EPP) play an important role in the evolution of mating systems (Petrie \& Kempenaers 1998; Clutton-Brock 2007). EPP are common in pair-living, or socially monogamous birds and mammals (see Table 1 for definitions used in this study), including humans, while genetic monogamy is a very rare phenomenon (Huck et al. 2014; Petrie \& Kempenaers 1998; Isvaran \& Clutton-Brock 2007). Among pair-living mammals which constitute up to $9 \%$ of mammal species, depending on the classification method (Lukas \& Clutton-Brock 2013; Opie et al. 2013) - strict genetic monogamy (no cases of EPP) has been reported for only seven species so far (Table 2). Four other species can be considered as "mostly" genetically monogamous, with the rate of EPP $<10 \%$. However, for most pair-living mammal species, genetic paternity data simply does not exist yet, and therefore our understanding of the frequency of genetic monogamy is very incomplete.

Rates of EPP vary substantially between species and populations and have been shown to be affected by various factors, such as, for example, intensity of male care, pair-bond strength and population density (Huck et al. 2014; Arct et al. 2015; Isvaran \& Clutton-Brock 2007; Brouwer et al. 2017). The intriguing question is why some individuals engage in mating with multiple partners while others do not. The advantages to males of engaging in extra-pair copulations (EPC) are well recognized, as males are expected to increase their fitness by 
increasing the number of mating partners as the result of their higher potential reproductive rate (Clutton-Brock 2007; Trivers 1972). However, in pair-living species with biparental care, potential reproductive rates and, consequently, levels of intra-sexual competition will be more similar for males and females (Clutton-Brock 2007). As a result, both sexes might be expected to gain benefits from engaging in EPC (Jennions \& Petrie 2000). These benefits might include insurance against social partner's infertility, maximizing genetic diversity among offspring or increasing offspring genetic quality by mating with individuals that are more genetically compatible or of higher genetic quality (reviewed in e.g. Brouwer \& Griffith 2019). One potential advantage of EPC to both sexes could be related to limitations in mate choice. In pair-living species with biparental care, especially in those with low mobility and low breeding density, mate choice can be highly constrained. First, mates become unavailable once paired. And second, individuals may face a conflict between choice for direct benefits (territory quality, intensity of paternal care) and indirect genetic benefits (partner genetic quality or compatibility). As a result of this constrained mate choice, individuals may end up paired to a genetically incompatible, closely related or low-quality partner. To escape these constraints, animals might seek EPC that would allow them to gain indirect benefits while still taking advantage of direct benefits provided by the social partner (Jennions \& Petrie 2000). This strategy has been demonstrated in various bird species (Foerster et al. 2003; Arct et al. 2015). In mammals, the evidence is much more limited. In Alpine marmots, Marmota marmota, and meerkats, Suricata suricatta, EPP rates were found to be higher in pairs where partners were more closely related (Cohas et al. 2008; Leclaire et al. 2013). But, to our knowledge, the positive relationship between partners' genetic similarity and EPP rates has been only demonstrated in one pair-living mammal species: in fat-tailed dwarf lemur, Cheirogaleus medius, females sharing more major histocompatibility complex (MHC)supertypes with their social partner were shown to engage in more EPC (Schwensow et al. 2008).

Table 1. Terminology used in this study, proposed in Huck et al. (2020) and based on the framework of Kappeler (2019).

Pair-living (who lives with whom): a type of social organization where one adult male and one adult female share a home range, possibly with their non-reproducing offspring. This is often referred to as "social monogamy".

Pair-bonded (who is affiliated with whom): a type of social structure where one adult male and one adult female have an affiliative relationship to the exclusion of other adults, as evidenced by behavioral, emotional, and endocrinological characteristics.

Sexual monogamy (who mates with whom): a type of social mating system where one adult male and one adult female have an exclusive mating relationship during at least one reproductive season. 
Genetic monogamy (who produces offspring with whom): a type of genetic mating system where one adult male and one adult female produce offspring exclusively with each other over a set of multiple births (at least one reproductive season for species that produce more than one infant per litter and over more than one consecutive reproductive seasons for species with singleton births)

Biparental care (who provides parental care): a type of care system where a mother and putative father regularly provide offspring care.

Table 2. List of genetically monogamous mammals with no extra-pair paternities (EPP) detected. Also included two predominantly genetically monogamous species with the proportion of EPP rate $<10 \%$.

\begin{tabular}{|c|c|c|c|c|}
\hline Species & Sample size & $\begin{array}{l}\text { Genotyping } \\
\text { method used }\end{array}$ & $\begin{array}{l}N \text { of EPP cases } \\
\text { found }\end{array}$ & References \\
\hline $\begin{array}{l}\text { Azara's owl } \\
\text { monkey (Aotus } \\
\text { azarae) }\end{array}$ & $\begin{array}{l}35 \text { offspring of } 29 \\
\text { family groups ( } 128 \\
\text { animals in total) }\end{array}$ & $\begin{array}{l}14 \text { microsatellite } \\
\text { loci }\end{array}$ & 0 & Huck et al. 2014 \\
\hline $\begin{array}{l}\text { Bornean gibbon } \\
\text { (Hylobates } \\
\text { muelleri) }\end{array}$ & $\begin{array}{l}4 \text { offspring of } 4 \text { family } \\
\text { groups (13 animals in } \\
\text { total) }\end{array}$ & $\begin{array}{l}16 \text { microsatellite } \\
\text { loci }\end{array}$ & 0 & $\begin{array}{l}\text { Oka \& Takenaka } \\
2001\end{array}$ \\
\hline $\begin{array}{l}\text { Kirk's dik-dik } \\
\text { (Madoqua kirkii) }\end{array}$ & $\begin{array}{l}12 \text { offspring of } 11 \\
\text { family groups (68 } \\
\text { animals in total) }\end{array}$ & $\begin{array}{l}7 \text { microsatellite } \\
\text { loci }\end{array}$ & 0 & $\begin{array}{l}\text { Brotherton et al. } \\
1997\end{array}$ \\
\hline $\begin{array}{l}\text { Coyotes (Canis } \\
\text { latrans) }\end{array}$ & $\begin{array}{l}96 \text { offspring of } 18 \\
\text { family groups ( } 236 \\
\text { animals in total) }\end{array}$ & $\begin{array}{l}12 \text { microsatellite } \\
\text { loci }\end{array}$ & 0 & $\begin{array}{l}\text { Hennessy et al. } \\
2012\end{array}$ \\
\hline $\begin{array}{l}\text { California mouse } \\
\text { (Peromyscus } \\
\text { californicus) }\end{array}$ & $\begin{array}{l}82 \text { offspring of } 22 \\
\text { complete groups, plus } \\
17 \text { offspring from } \\
\text { incomplete groups } \\
\text { (samples from father } \\
\text { or mother not } \\
\text { available) }\end{array}$ & $\begin{array}{l}\text { DNA } \\
\text { fingerprinting } \\
\text { using restriction } \\
\text { enzyme }\end{array}$ & 0 & Ribble 1991 \\
\hline $\begin{array}{l}\text { Malagasy giant } \\
\text { jumping rat } \\
\text { (Hypogeomys } \\
\text { antimena) }\end{array}$ & $\begin{array}{l}60 \text { offspring of } 28 \\
\text { family groups ( } 139 \\
\text { animals in total) }\end{array}$ & $\begin{array}{l}\text { Polymorphisms } \\
\text { of a major } \\
\text { histocompatibilit } \\
\text { y complex class } \\
\text { II gene DQA } \\
\text { using sequencing } \\
\text { and single- } \\
\text { strand } \\
\text { conformation } \\
\text { polymorphism } \\
\text { analysis }\end{array}$ & $\begin{array}{l}0 \text {, with } 3 \text { cases of } \\
\text { male and } 3 \text { cases } \\
\text { of female } \\
\text { replacement but } \\
\text { no litters sired by } \\
\text { multiple fathers }\end{array}$ & $\begin{array}{l}\text { Sommer \& Tichy } \\
1999\end{array}$ \\
\hline $\begin{array}{l}\text { Taiwan vole } \\
\text { (Microtus } \\
\text { kikuchii) }\end{array}$ & $\begin{array}{l}31 \text { offspring of } 20 \\
\text { family groups }\end{array}$ & $\begin{array}{l}10 \text { microsatellite } \\
\text { loci }\end{array}$ & $\begin{array}{l}0 \text {, with } 2 \text { cases of } \\
\text { female } \\
\text { replacement but } \\
\text { no litters sired by } \\
\text { multiple fathers }\end{array}$ & Wu et al. 2012 \\
\hline
\end{tabular}




\begin{tabular}{|c|c|c|c|c|}
\hline $\begin{array}{l}\text { Eurasian beaver } \\
\text { (Castor fiber) }\end{array}$ & $\begin{array}{l}\text { (a) } 18 \text { offspring of } 9 \\
\text { colonies plus } 6 \text { family } \\
\text { groups with only } \\
\text { adults ( } 38 \text { animals in } \\
\text { total) (Syrůčková et } \\
\text { al. } 2015 \text { ); } \\
\text { (b) } 166 \text { offspring of } \\
48 \text { family groups ( } 356 \\
\text { animals in total) }\end{array}$ & $\begin{array}{l}\text { (a) } 15 \\
\text { microsatellite } \\
\text { loci; } \\
\text { (b) } 27 \text { single } \\
\text { nucleotide } \\
\text { polymorphisms }\end{array}$ & $\begin{array}{l}\text { (a) } 0 \text {, with one } \\
\text { possible female } \\
\text { replacement but } \\
\text { no litters sired by } \\
\text { multiple fathers; } \\
\text { (b) } 9 \text {, } \\
\text { corresponding to } \\
\text { the EPP rate of } \\
5.4 \% \text {; } 7 \text { offspring } \\
\text { were sired by } \\
\text { neighboring males, } \\
\text { in } 2 \text { cases the } \\
\text { paternity could } \\
\text { not be assigned }\end{array}$ & $\begin{array}{l}\text { (a) Syrůčková et } \\
\text { al. 2015; } \\
\text { (b) Nimje et al. } \\
2019\end{array}$ \\
\hline Indri (Indri indri) & $\begin{array}{l}12 \text { offspring of } 7 \\
\text { family groups ( } 26 \\
\text { animals in total) }\end{array}$ & $\begin{array}{l}6 \text { microsatellite } \\
\text { loci }\end{array}$ & $\begin{array}{l}\text { 1, corresponding } \\
\text { to the EPP rate of } \\
8.3 \% \text {; social father } \\
\text { excluded as } \\
\text { genetic father but } \\
\text { no other male } \\
\text { indicated as likely } \\
\text { father }\end{array}$ & $\begin{array}{l}\text { Bonadonna et al. } \\
2019\end{array}$ \\
\hline $\begin{array}{l}\text { Golden-cheeked } \\
\text { gibbons } \\
\text { (Nomascus } \\
\text { gabriellae) }\end{array}$ & $\begin{array}{l}10 \text { offspring of } 6 \\
\text { family groups ( } 29 \\
\text { animals in total) }\end{array}$ & $\begin{array}{l}8 \text { microsatellite } \\
\text { loci }\end{array}$ & $\begin{array}{l}\text { 1, corresponding } \\
\text { to the EPP rate of } \\
10 \% \text {; a lone non- } \\
\text { territorial male } \\
\text { confirmed as } \\
\text { genetic father }\end{array}$ & $\begin{array}{l}\text { Kenyon et al. } \\
2011\end{array}$ \\
\hline $\begin{array}{l}\text { White-handed } \\
\text { gibbons } \\
\text { (Hylobates lar) }\end{array}$ & $\begin{array}{l}41 \text { offspring, } 27 \text { born } \\
\text { in pair-living groups } \\
\text { and } 15 \text { born in multi- } \\
\text { male groups ( } 89 \\
\text { animals in total) }\end{array}$ & $\begin{array}{l}12 \text { microsatellite } \\
\text { loci }\end{array}$ & $\begin{array}{l}3 \text {, corresponding } \\
\text { to the EPP rate of } \\
7.3 \% ; 2 \text { were sired } \\
\text { by neighboring } \\
\text { males, in } 1 \text { case } \\
\text { the paternity } \\
\text { could not be } \\
\text { assigned }\end{array}$ & $\begin{array}{l}\text { Barelli et al. } \\
2013\end{array}$ \\
\hline
\end{tabular}

Any mate choice, whether it is a choice for social or extra-pair partner, is expected to maximize not only direct fitness benefits, but also indirect (genetic) benefits, expressed as increased genetic quality of offspring. The closely related hypotheses of genetic compatibility and heterozygosity posit that individuals benefit from choosing a mate that will maximize offspring heterozygosity (Kempenaers 2007; Brown 1997; Zeh \& Zeh 1997). Thus, animals are expected to choose mates that are genetically unrelated or dissimilar at some fitnessrelated genes (e.g., MHC genes). An increase in offspring heterozygosity resulting from this disassortative mating is expected to increase offspring fitness, as indicated by links between individual heterozygosity and various fitness proxies, such as survival, reproductive success and parasite resistance (e.g., (Coltman et al. 1999; Foerster et al. 2003; Ortego et al. 2007); reviewed in (Kempenaers 2007)). In addition, irrespective of genetic compatibility, individuals might also benefit from choosing genetically higher-quality mates, i.e., those who 
carry "good genes" or are more heterozygous (Kempenaers 2007). With regard to heterozygosity, two opposing hypotheses have been suggested (Kempenaers 2007). First, heterozygosity may be expected to be positively correlated between mates, because less heterozygous individuals might be less successful in finding the best, i.e., more heterozygous partners. Alternatively, the correlation of heterozygosity between partners might be negative, because less heterozygous individuals need to compensate for their lower quality by choosing more heterozygous mates. Additionally, as heterozygous partners are expected to have higher fitness, they should additionally be more likely to provide direct benefits such as increased parental care, fertility or superior territory (Kempenaers 2007; Hoffman et al. 2007).

Mate choice based on heterozygosity was demonstrated in various species of birds and mammals. For example, in blue tits, Cyanistes caeruleus, heterozygosity was positively correlated between social mates, indicating that mating preferences were based on partner's heterozygosity (García-Navas et al. 2009). In Antarctic fur seals, Arctocephalus gazella, where females exert choice by moving across a breeding colony to visit largely stationary males, females were shown to move further to maximize the balance between male high heterozygosity and low relatedness (Hoffman et al. 2007). In many species, mate choice was shown to be based on MHC loci dissimilarity (e.g., fat-tailed dwarf lemur (Schwensow et al. 2008)) or, conversely, similarity (probably an adaptation to local pathogens, shown in, e.g., Malagasy giant jumping rat, Hypogeomys antimena, and European badgers, Meles meles (Sommer 2005; Sin et al. 2015)). Finally, relatedness-based mate choice, while demonstrated in some species, such as Antarctic fur seals, was not found in many other studied species, such as fat-tailed dwarf lemurs, blue tits and great reed warblers, Acrocephalus arundinaceus (García-Navas et al. 2009; Hansson et al. 2007; Sommer 2005; Schwensow et al. 2008).

The absence of relatedness-based mate choice in pair-living species, such as fat-tailed dwarf lemurs or blue tits, might seem particularly surprising, because the risk of inbreeding in such species is expected to be high due to constrained mate choice. In the absence of other options, or as a result of trade-offs between a choice for a good territory vs. a choice for unrelated/compatible partner, individuals might end up paired with close relatives. Therefore, pair-living animals may be expected to use relatedness-based mate choice to "actively" avoid pairings with closely related individuals (Leedale et al. 2020). However, pairings with close relatives might be also avoided "passively" by natal dispersal that can disrupt the associations of opposite-sex kin and thus prevent matings between them (Greenwood 1980). Dispersal was shown to be sufficient to avoid inbreeding or reach a certain level of genetic dissimilarity in many situations (García-Navas et al. 2009; Hansson et al. 2007; Huchard et al. 2010). It remains unclear, however, if dispersal has to be sex-biased 
to generate enough local genetic dissimilarity between breeding females and males to avoid inbreeding. In most mammals, males are the dispersing sex, because in polygynous mating systems, which are prevailing in mammals, males experience stronger intra-sexual competition for mates than females (Greenwood 1980; Dobson 1982). Following the same logic, mammals that mate monogamously or cooperatively with high levels of reproductive monopolization by a dominant pair are expected to have little or no sex bias in dispersal. This was found to be true in some mammals, such as the genetically monogamous Azara's owl monkey, Aotus azarae, where both sexes disperse, or cooperatively breeding meerkats, where dispersal is only slightly male-biased (Doolan \& Macdonald 1996; Fernandez-Duque 2009). However, in other mammals, e.g., genetically monogamous California mice, Peromyscus californicus, or socially monogamous greater white-toothed shrew, Crocidura russula, dispersal was found to be female-biased (Ribble 1992; Favre et al. 1997).

Here, we present a comprehensive study of the genetic mating system, mate choice and dispersal in a wild population of coppery titi monkeys, Plecturocebus cupreus. Titi monkeys (genera Callicebus, Plecturocebus, and Cheracebus) exhibit almost all the elements of the "monogamy package", such as pair living, strong long-term pair bonds, an exceptionally high level of male care (the infant is carried almost exclusively by the social father), territoriality, and sexual monomorphism (Van Belle, Fernandez-Duque, et al. 2016; Bicca-Marques \& Heymann 2013; Anzenberger 1988; Fernandez-Duque et al. 2013). The only missing component which has yet to be characterized is the genetic mating system. Titis are one of the very few mammalian taxa that exhibit both high level of male care and strong pair bonds, two characteristics shown to affect the rates of EPP in mammals (Huck et al. 2014). The examination of their mating system and the proximate mechanisms of its maintenance may, therefore, shed light on the evolution of social and genetic monogamy in mammals. In this study, we first examined the mating system of coppery titis using a set of 18 newly developed microsatellite loci that can be universally applied to Neotropical monkeys. Second, we tested for evidence of relatedness- and/or heterozygosity-based mate choice. Finally, to see if dispersal is sex-biased, we compared genetic relatedness and diversity patterns in adult females and males and performed spatial genetic analysis. Given consistent pair living, strong pair bonds and high levels of male care in coppery titis, we predicted them to be genetically monogamous or have a very low rate of EPP. Since the risk of inbreeding is expected to be especially high for long-lived pair-living species such as titis, we expected to find evidence for active inbreeding avoidance via mate choice and/or for heterozygosity-based mate choice. We predicted both sexes to disperse, as expected from a pair-living territorial mammal with biparental care. 


\section{Methods}

\section{Study site and study population}

The study was conducted at the Estación Biológica Quebrada Blanco in the north-eastern Peruvian Amazon ( $4^{\circ} 21^{\prime} \mathrm{S}, 73^{\circ} 09^{\prime} \mathrm{W}$ ) in June 2017 - September 2019. The study area consists mainly of undisturbed primary tropical rainforest of the "terra firme" type (not inundated during the rainy season) interspersed with small swampy areas. The home ranges of Groups 2,3 , and 13 also included land strips of secondary growth along the river that can be inundated for a few days during the height of the rainy season. The spatial gap between Groups 1, 6, 14, and 7 (Fig. 1, 2) is partly occupied by a secondary forest (abandoned buffalo pasture regenerating since 2000) that is avoided by titi monkeys (Kulp \& Heymann 2015). The spatial gap between Groups 8-9 and the remaining groups (Fig. 1,2) was a result of study logistics (proximity to camp buildings), not a lack of titi groups in this area.

Study individuals belonged to 14 family groups (Supplementary Table S1, Fig. 1), seven of which (Groups 1-7) were also subject to behavioral studies conducted in June-December 2017 and June-December 2018 (Dolotovskaya, Walker, et al. 2019; Dolotovskaya \& Heymann 2020; Dolotovskaya, Flores Amasifuen, et al. 2019). Between the periods of behavioral data collection, the groups were monitored for 2-3 days per month, and genetic samples were collected continuously from the beginning of the study until September 2019. Group 1 had been habituated to the presence of human observers and studied intermittently since 1997; the other groups were habituated during this study. We individually identified all study animals based on the combination of body size, tail shape and colouration, genitalia shape, and natural marks. To control for possible misidentification of animals in the field, we genotyped study individuals from 2-3 independent samples (only one individual, a juvenile from Group 10, was genotyped from just one sample; see Discussion). We also used a PCRbased sexing assay (Di Fiore 2005) to confirm reported sex (and to sex young individuals for whom sex could not be identified in the field).

Home ranges of study groups (Fig. 1, 2) were estimated using the 95\% fixed kernel density method with ArcGIS Desktop 10.6 (ESRI) on the basis of GPS points collected during group follows at 10 min intervals with a GPS unit (Garmin GPSMAP 62s or 64s; N = 19,456 GPS points, mean per group = 1497 GPS points). Home range sizes varied from 3.6 to 12.98 ha (Supplementary Table S1).

Titis typically give birth to a single infant once a year (Van Belle, Fernandez-Duque, et al. 2016; Bicca-Marques \& Heymann 2013; Souza-Alves et al. 2019). In our study population, most of the births occurred between October and February and only one occurred in June (Supplementary Table S1). As the offspring disperse at the age of 2-4 years (Van Belle, 
Fernandez-Duque, et al. 2016; Bicca-Marques \& Heymann 2013; Souza-Alves et al. 2019), the pedigree in our study comprised up to 5 generations of offspring per group (Supplementary Table S1).

\section{Fecal sample collection and DNA extraction}

We collected fecal samples from 41 individuals (3-15 samples per individual) living in 14 family groups, including 18 putative offspring of 9 family groups (1 to 5 offspring per group). Five other groups either did not have offspring during the study period (or they had disappeared before we could collect samples) or the samples could not be collected because the offspring were still very young and thus their defecations too diminutive to be detected. Also due to differential habituation to the presence of humans, for some groups we could not obtain samples from all group members. For those groups that were habituated in the beginning of the study period, we collected samples from offspring from several consecutive years.

Fecal samples were collected immediately after an identified individual was seen defecating. We dried the samples in $15 \mathrm{~mL}$ falcon tubes containing silica gel beads (Carl Roth, Karlsruhe, Germany) and stored them at ambient temperature, replacing the silica beads when necessary, until shipping to Germany.

We extracted DNA (at least two samples per individual for all animals except one offspring of Group 10; see Results for more details) from ca. $200 \mathrm{mg}$ of feces using the Macherey-Nagel NucleSpin $\odot$ DNA stool kit with a final elution of the DNA in $50 \mu \mathrm{L}$ elution buffer. DNA concentration of the extracts was measured using a NanoDrop Spectrophotometer (ND-1000, PEQLAB Biotechnologie GmbH) and a Qubit Fluorometer (Thermo Fisher).

\section{Microsatellite genotyping}

As published microsatellite loci for titi monkeys (Mendoza et al. 2015; Martins 2015; Menescal et al. 2009) revealed unreliable results for our study species, we established a new set of 27 di-repeat microsatellite loci that can be universally applied to Neotropical primates (details are described in Supplementary Materials and Supplementary Tables S3-4). To simplify library preparation for genotyping by sequencing, we added adapter nucleotide sequences to the $5^{\prime}$ end of the locus-specific primers.

We amplified all 27 loci in a single multiplex PCR using the Qiagen Multiplex PCR Kit (Qiagen) with a total volume of $25 \mu \mathrm{L}$ and containing $12.5 \mu \mathrm{L} 2 \mathrm{x}$ Multiplex Master Mix, $1 \mu \mathrm{L}$ of primer pool ( $0.2 \mu \mathrm{M}$ of each primer), $1 \mu \mathrm{L}$ of DNA extract (ca. $200 \mathrm{ng}$ total DNA) and $10.5 \mu \mathrm{L}$ of RNasefree water. Amplifications were performed with initial denaturation at $95^{\circ} \mathrm{C}$ for $15 \mathrm{~min}, 40$ cycles of denaturation at $94^{\circ} \mathrm{C}$ for $30 \mathrm{sec}$, annealing at $57^{\circ} \mathrm{C}$ for $1.5 \mathrm{~min}$, extension at $72{ }^{\circ} \mathrm{C}$ for 
$1 \mathrm{~min}$ and a final extension at $60^{\circ} \mathrm{C}$ for $30 \mathrm{~min}$. PCR products were checked on $1.5 \%$ agarose gels together with non-template controls. To prevent false homozygosity due to allelic dropout, we repeated each multiplex reaction three times (Barbian et al. 2018). In some samples, the total multiplex reaction with all 27 loci yielded low number of sequencing reads; in these cases, we additionally amplified the loci in three separate multiplex reactions with the following primer pools: chr01b-chr07a, chr08a-chr12a, chr12b-chrXa, as this method usually yielded more reads (see Supplementary Materials for details). The reactions and PCR conditions for three separate multiplex reactions were the same as for the total multiplex reaction.

Following amplification, we pooled $5 \mu \mathrm{L}$ of each multiplex PCR product (or of each PCR product of three separate multiplex reactions), purified the pooled products with the Monarch PCR \& DNA Cleanup Kit (New England BioLabs) and quantified them using Qubit Fluorometer (Thermo Fisher). To prepare sequencing libraries, we performed indexing PCRs using Kapa HiFi Hotstart ReadyMix PCR Kit (Roche) with a total volume of $25 \mu \mathrm{L}$ containing $12.5 \mu \mathrm{L} 2 \mathrm{x}$ Kapa HiFi Hotstart ReadyMix, $1 \mu \mathrm{L}(0.5 \mu \mathrm{M})$ of each indexing primer (containing individual barcodes) and 100 ng purified PCR product. Indexing PCRs were done with an initial denaturation step at $98^{\circ} \mathrm{C}$ for $45 \mathrm{sec}$, followed by 4 cycles of denaturation at $98^{\circ} \mathrm{C}$ for $15 \mathrm{sec}$, annealing at $62^{\circ} \mathrm{C}$ for $30 \mathrm{sec}$ and extension at $72{ }^{\circ} \mathrm{C}$ for $30 \mathrm{sec}$, and a final extension step at $72^{\circ} \mathrm{C}$ for $1 \mathrm{~min}$. Full-length libraries were purified with the Monarch PCR \& DNA Cleanup Kit (New England BioLabs) and quantified using Qubit Fluorometer (Thermo Fisher). Fragment sizes and molarities were quantified using a Bioanalyzer 2100 (Agilent Technologies). Libraries were diluted to $10 \mathrm{nM}$ and then pooled and sequenced using Miseq Reagent Kit v2 with PhiX DNA (Illumina) added on the MiSeq system (Illumina). Sequencing was performed with 51 forward and 251 reverse read cycles. Only the reverse reads were used for further analysis, while forward reads were only used for MiSeq quality control. To control for laboratory mistakes, we genotyped each sample twice, leading to at least four genotypes per individual.

After sequencing, the samples were demultiplexed using MiSeq Reporter software and then processed using the CHIIMP analysis pipeline (Barbian et al. 2018). The CHIIMP pipeline calls alleles by first producing unique sequences with relevant attributes (read counts, sequence length, etc.) for each MiSeq sequence file, querying the sequences for potential PCR artifacts, such as stutter sequences, and then removing all sequences that do not match the locus attributes. All alleles called by CHIIMP were manually checked to validate the results and to correct automated allele calling for those loci that contain "wobble" positions in the primer sequences and are incorrectly processed by CHIIMP. We used a cutoff of 250 reads. 
Additionally, we accepted alleles if they yielded $>100$ reads in at least three genotypes obtained per individual. Alleles with $<100$ reads were not called.

Of 27 loci, nine either consistently failed to amplify in our study animals (chr06b, chr11f, chr16b) or proved to be monomorphic (chr02a, chr02b, chr04a, chr10b, chr12a, chr13b) and were excluded from further analysis. The final set consisted of 18 loci, including 17 autosomal and one X-linked locus (chrXa) (Supplementary Table S5). All animals were genotyped at a minimum of 14 loci (16.8 loci on average), and the mean number of alleles per locus was 8.9. We checked all loci for the presence of null alleles, allelic dropout and stuttering using MicroChecker 2.2.5 (Van Oosterhout et al. 2004). We assessed Hardy-Weinberg equilibrium (HWE) and calculated observed and expected heterozygosity with PopGenReport 2.2.2 (Adamack \& Gruber 2014). Since the presence of family structure can cause deviations from HWE and bias population genetic analyses, especially in monogamous species, we only included adults in this analysis. The analysis indicated that the population was in HWE. Two loci, chr01b and chr21a, departed from HWE, likely due to the presence of relatives in a study group and/or small sample size.

One of these two loci, chr01b, also showed evidence of null alleles. As the locus did not show any mismatches for the known mother/offspring dyads (see below), we ran all further analyses using two sets of data, one with the full set of loci and another one with locus chr01b excluded. Since the results from these two sets did not differ substantially, we present all further results only for the reduced data set.

\section{Mitochondrial DNA (mtDNA) genotyping}

We genotyped all individuals at the hypervariable region I of the mitochondrial control region using primers 5'-TACCTCGGTCTTGTAAACCG-3' and 5'-AGGTAGGAACCAGATGCCG-3', newly designed on the basis of mitochondrial genomes of Neotropical primates available in GenBank. PCR reactions with a total volume of $30 \mu \mathrm{l}$ contained $1 \mathrm{U}$ BiothermTaq 5000 (Genecraft), $1 \mathrm{x}$ reaction buffer, $0.16 \mathrm{mM}$ of each $\mathrm{dNTP}, 0.33 \mu \mathrm{M}$ of each primer and ca. $100 \mathrm{ng}$ total DNA. PCRs were performed with initial denaturation at $95^{\circ} \mathrm{C}$ for $2 \mathrm{~min}, 40$ cycles of denaturation at $95^{\circ} \mathrm{C}$ for $1 \mathrm{~min}$, annealing at $58^{\circ} \mathrm{C}$ for $1 \mathrm{~min}$, extension at $72{ }^{\circ} \mathrm{C}$ for $1 \mathrm{~min}$ and a final extension at $72{ }^{\circ} \mathrm{C}$ for $5 \mathrm{~min}$. PCR products were run on $1 \%$ agarose gels, excised from the gel and then purified with the Monarch DNA Gel Extraction Kit (New England BioLabs) and sequenced on an ABI 3130xL sequencer using both amplification primers and the BigDye Cycle Sequencing Kit (Applied Biosystems). Sequence electropherograms were checked with 4Peaks 1.8 (https://nucleobytes.com/4peaks/index.html) and manually edited and assembled in SeaView 4.5.4 (Gouy et al. 2010); all haplotypes were 567 bp long. 
As X-linked loci are haploid in males and cannot be treated in the analyses in the same way as autosomal loci, all the following statistical tests were performed using the set of 16 autosomal loci for both sexes. The data for the X-linked locus chrXa was used separately to manually check for allelic mismatches between candidate parents and offspring in the parentage analyses.

\section{(1) Parentage analyses}

Parentage was assigned using Cervus 3.0 (Kalinowski et al. 2007). Cervus compares likelihood ratios of the two most likely candidate parents and assigns parentage based on statistical thresholds generated during the simulation analysis. For Cervus analysis, we used a simulation of 100,000 offspring, an error rate of $0.01,90 \%$ relaxed and $95 \%$ strict confidence level, and accounted for relatedness of candidate mothers and fathers. Relatedness was calculated with the R package related 1.0 (Pew et al. 2015) using Wang's estimator $r$ (Wang 2002). This estimator was chosen because it performed best in simulations, showing the highest correlation between observed and expected values for our set of loci. We also specified a proportion of candidate fathers sampled as 0.85 to allow for unsampled males in adjacent territories and potential floaters. Additionally, we used Colony 2.0.6.5 (Jones \& Wang 2010) to verify parentage assignments from Cervus. Unlike Cervus, Colony reconstructs a full pedigree, inferring sibship and parentage among individuals by comparing the likelihood of different clusters of individuals and maximizing group rather than pairwise likelihoods. For this analysis, we used an error rate of 0.01 , male and female polygyny, and a sibship size prior of 1.6 , calculated as the average number of offspring per family group in our study population.

For both Cervus and Colony analyses, the set of candidate fathers included all sampled adult males plus the oldest subadult male from Group 6 that had dispersed from his natal group in the beginning of the study and could have sired offspring by the end of the sampling period. The set of candidate mothers included all adult females that shared their mtDNA haplotype with candidate offspring. For seven offspring (Supplementary Table S1), the mothers were known because they were seen nursing them. To test the reliability of our parentage estimates, we ran the analyses twice, with and without the respective set of known motheroffspring pairs. Combined non-exclusion probability for the set of 16 autosomal loci (with chr01b excluded) was $9.9 \times 10^{-5}$ for the first parent, $3.4 \times 10^{-7}$ for the second parent, and $9.0 \times 10^{-}$ 12 for the parent pair, calculated using Cervus.

\section{(2) Relatedness-based mate choice}

To test if titis avoid mating with related individuals, we compared relatedness between real and randomly generated mating partners using the pairwise relatedness estimator 
implemented in STORM (Frasier 2008). First, STORM calculates the relatedness of real mating pairs using the estimator of $\mathrm{Li}$ ( $\mathrm{Li}$ et al. 1993), with each locus weighted using the method described in (Lynch \& Ritland 1999; Van de Casteele et al. 2001). Then, the program calculates the expected relatedness of mating pairs if the mating is random with respect to relatedness; this is done by generating mating pairs from female and male breeding pools over 1000 iterations and averaging the relatedness values for each iteration. The obtained distribution is then compared to the averaged relatedness of real mating pairs. Our sample included ten real mating pairs, and the breeding pool consisted of 12 females and 12 males. This included all sampled adults and the oldest subadult male from Group 6.

\section{(3) Heterozygosity-based mate choice}

To test if titis show any heterozygosity-based mating pattern, we compared individual heterozygosity levels between pair mates. To estimate individual heterozygosity, we calculated homozygosity by loci (HL), a microsatellite-derived measure that weights the contribution of each locus to the homozygosity value depending on their allelic variability, implemented in R function GENHET 3.1 (Coulon 2010). To test if HL is correlated between pair mates, we used a two-tailed Pearson correlation analysis.

\section{(4) Dispersal patterns}

To examine if dispersal distances differ between sexes, we compared the diversity of mtDNA haplotypes, relatedness, and heterozygosity among adult females and males. MtDNA haplotype and nucleotide diversity was calculated and compared using a permutation test implemented in R function genetic_diversity_diff 1.0.6 ((Alexander et al. 2016); available from https://github.com/laninsky/genetic diversity diffs). We included 12 sampled adult females and 12 adult males in this analysis, plus two females that could not be sampled but whose haplotypes were inferred from the haplotypes of their offspring (the adult female of Group 4, who supposedly had been replaced before the study period, and the adult female of Group 9, who was present during the study period but could not be sampled). Relatedness among females and among males was calculated using Wang's estimator $r$ and then compared using 1000 bootstrapping samples in Coancestry 1.0.1.9 (Wang 2011). In this analysis, as well as in the tests described below, we included 12 sampled adult females and 12 males. Individual heterozygosity was calculated using HL estimator (homozygosity by locus, see above) and compared between sexes using a paired t-test.

To evaluate spatial genetic structure, we conducted a spatial autocorrelation analysis following Smouse and Peakall (Smouse \& Peakall 1999) in PopGenReport 2.2.2 (Adamack \& Gruber 2014), separately for adult females and males. The analysis calculated the correlation coefficient $r$ between pairwise genetic distances, calculated using microsatellite genotypes 
with the method of Smouse and Peakall (Smouse \& Peakall 1999), and pairwise spatial distances, for each distance class. The coefficient $r$ is bound between -1 and 1 and has a mean of zero when there is no correlation. As a measure of spatial distances, we used distances between centroids of home ranges calculated with ArcGIS Desktop 10.6 (ESRI). These distances varied from 215 to $3200 \mathrm{~m}$.

To further evaluate spatial genetic structure in females, we conducted a test similar to spatial autocorrelation analysis using mtDNA haplotype distances, correlating the number of nucleotide differences between haplotypes with spatial distances. For this test, if a spatial genetic structure is present, a positive correlation between haplotype and spatial distances is expected. We used Mantel tests with 10,000 permutations in R package ecodist (Goslee \& Urban 2007).

\section{Results}

Are titis genetically monogamous?

Our analyses did not indicate any cases of EPP. In all cases of assigned paternity (17 offspring in 9 social groups, 1 to 5 offspring per group; Fig. 1, Supplementary Table S1), social fathers were identified as genetic fathers of all offspring in their respective family groups. In one case, paternity remained unassigned. The juvenile male from Group 10 had three mismatches with the adult male of the group, and Delta score calculated by Cervus (the difference between the likelihood ratios for two most likely candidate parents) was zero, indicating both this male and the adult male of Group 8 as most likely fathers. At one of the loci with mismatches (chr09a), the juvenile was homozygous, likely resulting from allelic dropout or genotyping error, at two other loci (chr07a, chr08a), the juvenile was heterozygous, so we can only suggest that it was a result of a genotyping error. Unfortunately, this juvenile had the minimum number of typed loci among all the sampled animals and also was the only individual in our dataset for whom we only had one fecal sample collected. Therefore, we could not control for the errors using another sample like we did for all other individuals. 


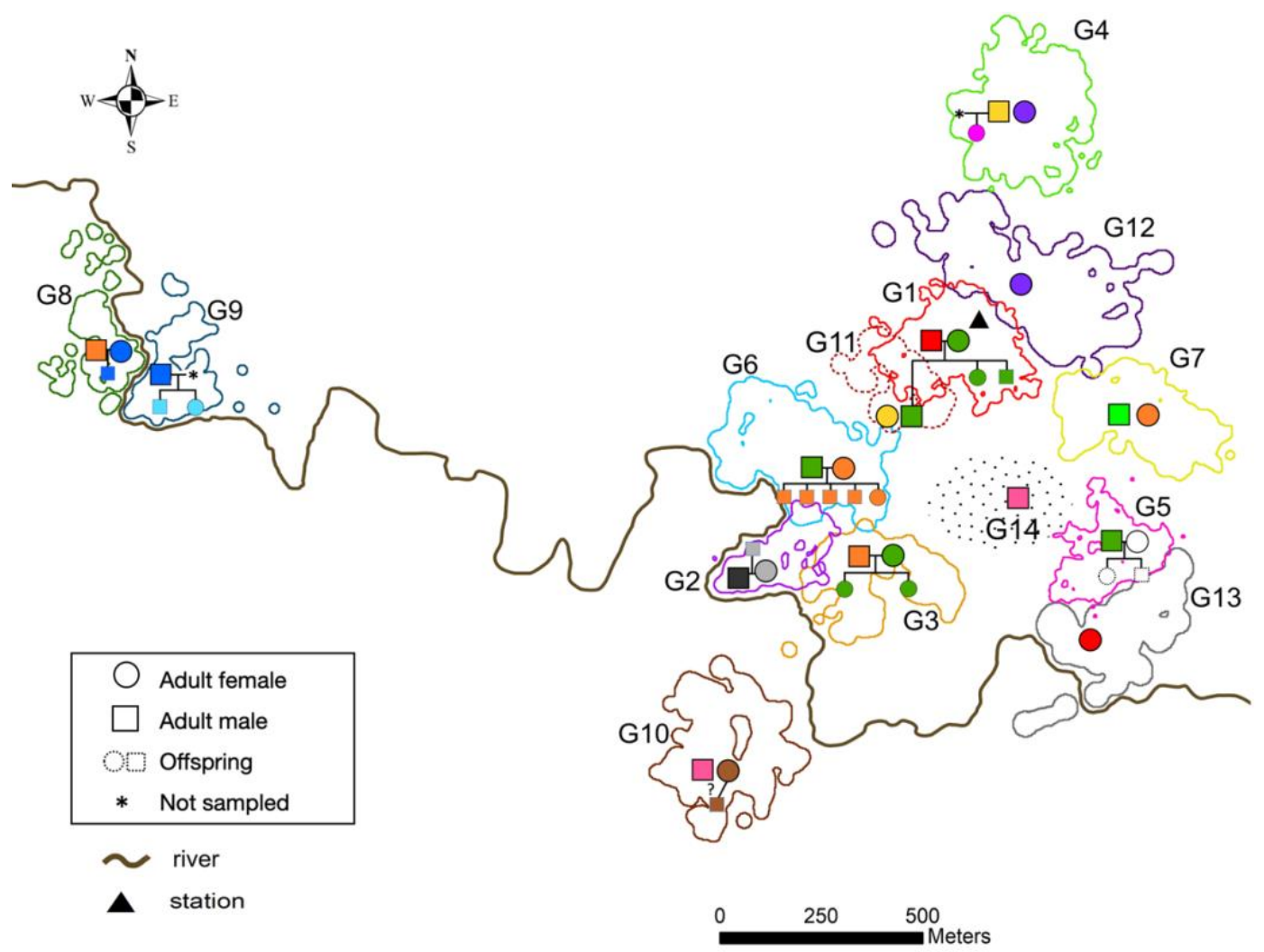

Fig. 1. Home ranges, mtDNA haplotypes, and parentage for sampled individuals within study groups. Circles and squares with continuous outline represent adult females and males, respectively; smaller circles and squares with dotted outline represent female and male offspring, respectively. The colors of circles and squares represent different mtDNA haplotypes. Home ranges of study groups were estimated using the $95 \%$ fixed kernel density method with ArcGIS Desktop 10.6 (ESRI; https://desktop.arcgis.com) (see more details in Methods). The home range of Group 14 is depicted as dotted ellipse because we did not have enough GPS data to reliably estimate its home range. The home range of Group 11 is depicted as dotted line because this newly established territory was most likely not permanent and bound to shift later (see Supplementary Materials for details of the dispersal event and Methods for more details on the habitat). The map was created in ArcGIS Desktop 10.6 and modified with Inkscape 1.0.1 (https://inkscape.org/).

Apart from the three mismatches in the case of the unassigned paternity, we found only two cases of allelic mismatches (Supplementary Table S1). Father-daughter dyad from Group 1 had a mismatch at locus chr10a. Since the daughter was homozygous at this locus, this was most likely a result of allelic dropout or genotyping error. Father-daughter dyad from Group 9 had a mismatch at locus chrXa; the daughter was not homozygous at this locus but considering the high likelihood of parentage given by the other loci, we assumed that this mismatch was due to a genotyping error.

Of eight sampled social mothers, seven were identified as genetic mothers of all offspring in their family groups (17 offspring in 7 groups, 1 to 5 offspring per group). One inferred case of female replacement was detected, as the adult female of Group 4 was not identified as the genetic mother of the group's juvenile offspring; they did not share the mtDNA haplotype and had 11 allelic mismatches. No other female in our sample was identified as the most likely 
mother for this offspring or shared a mtDNA haplotype with it. The social father of this juvenile was indicated as the genetic father.

All assignments were made with a 95\% confidence level in Cervus software and confirmed with Colony software (Supplementary Table S1). The assignments did not change when the set of known mother-offspring pairs was excluded from the priors. Colony also yielded strong support for full-sib relationships between all offspring from the same groups, confirming correct parentage assignments.

Is mate choice based on relatedness or heterozygosity?

We found no evidence for relatedness-based mate choice. There was no difference between relatedness of real mating pairs and randomly generated mating pairs $(-0.048$ vs. $-0.021, p=$ $0.565 ; \mathrm{n}=10$ pairs, breeding pool of 12 females and 12 males). Likewise, we found no evidence for heterozygosity-based mate choice, as homozygosity by loci (HL) was not significantly correlated between pair mates $(r=-0.527, n=10$ pairs, $p=0.118)$.

Despite the lack of evidence for active inbreeding avoidance via mate choice, relatedness (Wang's $r$ ) between mating partners was generally low, averaging -0.033 , and none of the pair mates shared the same mtDNA haplotype (Supplementary Table 1, Fig. 1). Only in one pair were the partners found to be second-degree kin (Group 6, $r=0.285$ ). The mtDNA haplotype network (Fig. 2) showed no clear pattern of haplotype similarity between pair mates: some had closely related haplotypes (e.g., Groups 4, 5, 9), while others had only distantly related haplotypes (e.g., Groups 1,11).

Do both sexes disperse and does one sex disperse further than the other?

Our results indicate that both sexes dispersed similar distances. There were no significant differences between adult females and males in mean mtDNA haplotype diversity (0.945 in females, 0.924 in males, permutation test $p=0.766)$, mtDNA nucleotide diversity $(0.027$ in females, 0.029 in males, permutation test $p=0.699)$, mean relatedness $r(-0.013$ in females, 0.056 in males, mean difference -0.040 , lying within the $95 \%$ confidence interval $(-0.048-$ 0.054 ) obtained by bootstrapping) or mean heterozygosity HL ( 0.184 in females, 0.216 in males, paired t-test $\mathrm{p}=0.438$ ).

We did not find evidence for spatial genetic structure in either sex, suggesting that both sexes likely dispersed over varying distances. The correlation between genetic and spatial distances was not significant for either sex, as the $95 \% \mathrm{CI}$ of autocorrelation $\mathrm{r}$ values overlapped zero for all distance classes (Supplementary Materials Table 2, Fig. S1). The correlation between mtDNA haplotype distances and spatial distances in females was not significant either (Mantel correlation $=0.048, \mathrm{n}=91$ dyads, right-tailed $\mathrm{p}=0.342$ ). 


\section{Discussion}

The link between mating system, mate choice and dispersal has rarely been studied in pairliving mammals. Here, we demonstrated that coppery titi monkeys in our study population are mostly genetic monogamous, as we did not find evidence for EPP. We also did not find evidence for relatedness- or heterozygosity-based mate choice. Despite the absence of evidence for active inbreeding avoidance via mate choice, pair mates in our study population had low average relatedness. This finding suggests that natal dispersal can generate sufficient level of genetic dissimilarity between females and males to render both active inbreeding avoidance and EPC less necessary.

Coppery titis are only the second primate species and the seventh pair-living mammal with no evidence of EPP found in a study with an adequate sample size (the study on Bornean gibbon was based on just four infants from four family groups (Oka \& Takenaka 2001), Table 1). The absence of EPP in titis is not unexpected, as they are consistently pair-living, pair mates spend most of the day within a few meters from each other, sleep together at night and engage in frequent joint visual displays and duetting at the territorial borders (Dolotovskaya, Walker, et al. 2019; Fernandez-Duque et al. 2013; Kinzey \& Wright 1982; Spence-Aizenberg et al. 2016; Kinzey \& Robinson 1983). This high level of proximity and coordination should make mate guarding easy and effective enough to prevent EPC.

The opportunities to find extra-pair mates are likely limited, too. The home ranges of our study groups have very little overlap (mean 1.4\% of pairwise overlap between neighboring groups (0-4.7), unpublished data; Fig. 1), and to find extra-pair mates, individuals would need to intrude into the neighboring home ranges, risking aggression from the same-sex residents. Another way to obtain EPC could be mating with floaters, solitary non-territorial individuals ranging over a wide area after having dispersed from their natal groups. There is accumulating evidence for the importance of floaters in population dynamics of both birds and mammals (Penteriani et al. 2011; Fernandez-Duque \& Huck 2013). For example, in Azara's owl monkeys who are very similar to titis in all aspects of their social system, mated individuals experience intense intra-sexual competition from floaters of both sexes (Fernandez-Duque \& Huck 2013; Huck \& Fernandez-Duque 2012a). However, the evidence from Azara's owl monkeys and many other bird and mammal species indicate that floaters do not copulate with the mated animals as often as might be intuitively expected, and EPP are attributed to the neighboring individuals in most cases (e.g., (Barelli et al. 2013; Nimje et al. 2019; Petrie \& Kempenaers 1998); but see (Cohas et al. 2006; Kenyon et al. 2011)). In titis, only anecdotal reports of replacements by intruders exist (Lawrence 2007; Rodman \& Bossuyt 2007), but given the difficulty of detecting floaters, it is possible that they are present 
in titi populations, too. However, given the high levels of proximity and coordination between pair mates, EPC with the floaters are probably not easier to obtain than EPC with the neighboring individuals. Furthermore, EPC, whether with floaters or neighboring animals, might be costly, with the risks including the higher probability of acquiring sexually transmitted diseases and, for females, the retaliatory withholding of parental care by males (Westneat \& Stewart 2003; Poiani \& Wilks 2000).

Opportunities for EPC are also affected by population density, with the lower densities making the encounters between individuals and, consequently, EPC less likely (Westneat 1990). The positive relationship between population density and EPP rates was demonstrated, e.g., in Eurasian beavers, Castor fiber, and in many bird species (Brouwer et al. 2017; Westneat \& Sherman 1997; Nimje et al. 2019; Syrůčková et al. 2015). At our study site, population density was estimated at 34 individuals $/ \mathrm{km}^{2}$ (unpublished data). This lies within the average range of values reported from behavioral studies for undisturbed populations of titis (26-57 individuals $/ \mathrm{km}^{2}$ ); for comparison, reported population density in forest fragments can be as high as 369 individuals $/ \mathrm{km}^{2}$ (Bicca-Marques \& Heymann 2013; Fernandez-Duque et al. 2020; Dacier et al. 2011). The relatively low density at our study site likely limited the opportunities for EPC. It should be mentioned, however, that for a population of white-tailed titi, Plecturocebus discolor, from undisturbed habitat, a preliminary analysis reported three cases of EPP in a sample size of 16 offspring, although these data has not been published yet (Van Belle, Martins, et al. 2016). The density of this population (57 individuals $/ \mathrm{km}^{2}$ ) was slightly higher than that of our study population, the home ranges were on average smaller (7.2 vs. $5.0 \mathrm{ha}$ ) and the percentage of home range overlap was larger (1.4\% vs. 4.8\%) (Fernandez-Duque et al. 2020), possibly accounting for the occurrence of EPP.

Although in all cases of assigned paternities the social fathers were identified as genetic fathers for the group offspring (17 offspring born in 9 groups, up to 5 offspring generations per group), we cannot fully exclude the possibility of a low EPP rate in our study population. First, for one juvenile (Group 10), paternity remained unassigned, as neither social father nor any other male from our sample was identified as the most likely father. While this case could be classified as neither extra- nor intra-pair paternity with confidence, it remains possible that this juvenile was sired by an unsampled extra-pair male. In this case, the EPP rate in our study population would be $6 \%$. Alternatively (if we assume that the social father is indeed not the genetic father of the juvenile), this case could be the result of a male replacement in a group. Adult replacements are known to happen in titis, with the breeding positions vacated after the disappearance (presumable deaths) of adults being occupied by same-sex immigrants (Van Belle, Fernandez-Duque, et al. 2016). Replacements can create groups that 
do not consist of biological parents and their offspring, leading to the apparent deviations from genetic monogamy even in the absence of EPC. As Group 10 was only habituated shortly before the genetic sample collection and no older offspring were present in it, we could not reconstruct its demographic history. Our data indicates that adult replacements do happen in our study population. The adult female of Group 4 was not identified as the genetic mother of the group's juvenile offspring, while the adult male was indicated as the genetic father. When we started following this group, the juvenile was estimated to be 7-8 months old based on its body size and the fact that it walked independently (juvenile titis start to walk on their own most of the time at the age of ca. 4.5 months (Jantschke et al. 1995)). Lactation in titis lasts ca. 6.5 months (Valeggia et al. 1999), and we did not see the female nursing. Therefore, we assume that the female replacement must have happened within ca. 2 months before we started following the groups, after the juvenile had been weaned.

Second, for the sample size of 17 offspring, an upper limit of 95\% confidence interval of EPP level (maximum possible EPP level for a given sample size, assuming no EPP has been found) will be $16.2 \%$. This value is calculated following Brotherton et al. (Brotherton et al. 1997) as $1-(1-x)^{n}=y$, where $x$ is the maximum possible EPP level, $n$ is the sample size (17), and $y$ is the probability of producing at least one extra-pair offspring ( 0.95 for $95 \%$ confidence). The value of $16.2 \%$ is a product of the sample size and does imply that there is $16.2 \%$ EPP rate in our study population. To narrow down the confidence interval to at least $5 \%$ of EPP, we would need a sample size of 58 offspring, which is difficult to achieve in a reasonable period in a secretive arboreal primate with slow life history, living in pairs and giving singleton births only once a year.

Contrary to our predictions, we did not find evidence for relatedness- or heterozygositybased mate choice in our study population. Interestingly, despite the absence of evidence for active inbreeding avoidance via mate choice, the pair mates in our study population were on average not related (mean Wang's $r=-0.033$ ) and never shared the same mtDNA haplotype (Supplementary Table S1, Fig. 2). Only in one case the pair mates were second-degree kin with $r=0.285$. Low relatedness between mating partners in the absence of active inbreeding avoidance was demonstrated in many other populations of mammals and birds, e.g., grey wolves, Canis lupus, arctic foxes, Vulpes lagopus, great reed warblers, and blue tits (Hansson et al. 2007; Foerster et al. 2006; Geffen et al. 2011). In fact, active inbreeding avoidance via mate choice, although demonstrated in some birds and mammals (e.g., (Hoffman et al. 2007; Leedale et al. 2020)), has not been found in most pair-living species (García-Navas et al. 2009; Schwensow et al. 2008; Hansson et al. 2007; Sommer 2005); reviewed in (Jamieson et al. 2009). It has been suggested that in most situations, dispersal may be sufficient to avoid 
inbreeding (Hansson et al. 2007). By disrupting close-kin associations, dispersal can make the probability of encountering close kin relatively low, rendering active inbreeding avoidance via mate choice unnecessary (Jamieson et al. 2009). In such cases, kin discrimination mechanisms might fail to evolve, and low inbreeding levels that will occasionally occur in such systems will be tolerated (Jamieson et al. 2009).

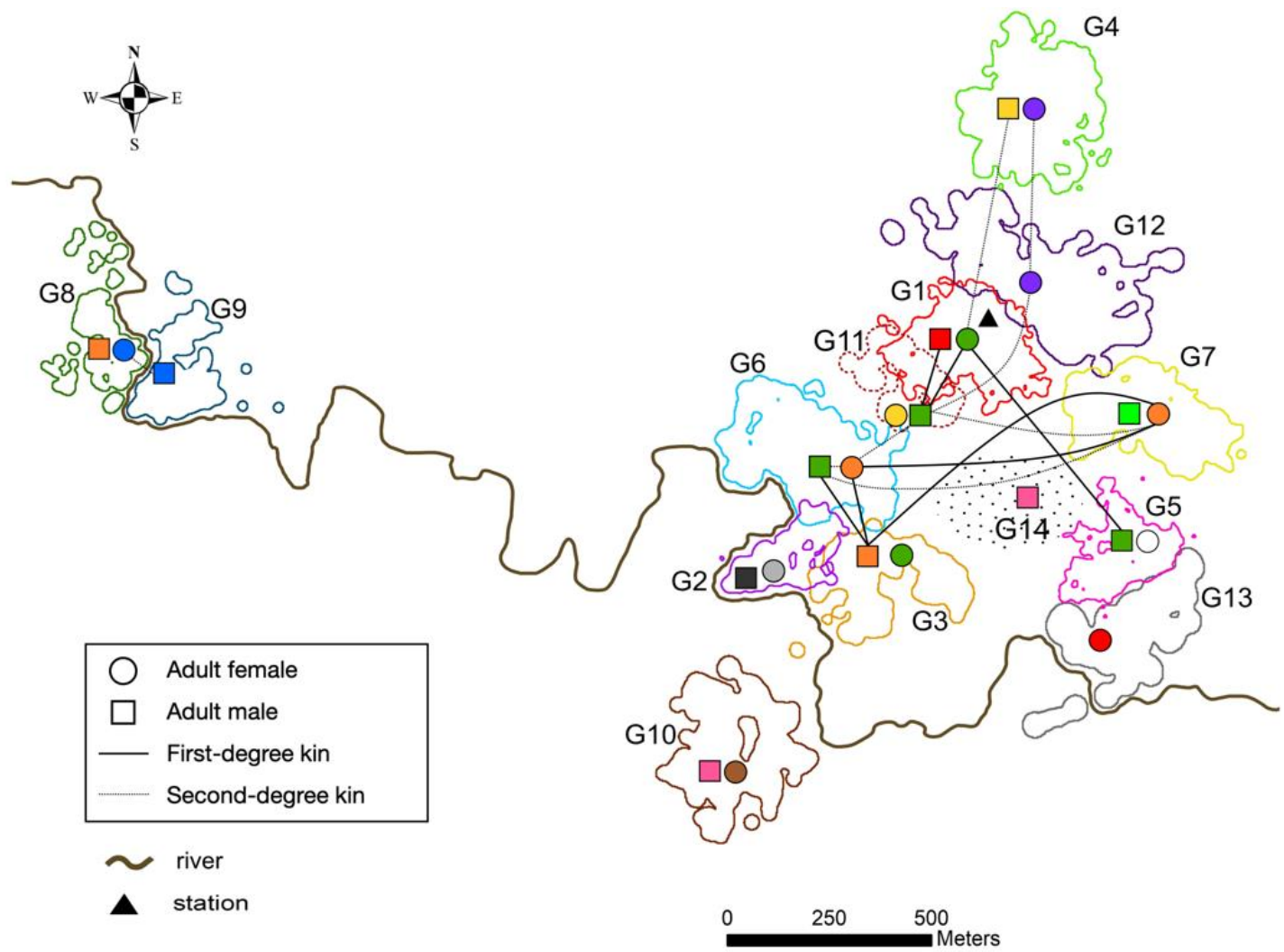

Fig. 2. Home ranges, relatedness and mtDNA haplotypes of adult females (circles) and males (squares) sampled in this study. Home ranges of study groups were estimated using the $95 \%$ fixed kernel density method with ArcGIS Desktop 10.6 (ESRI; https://desktop.arcgis.com) (see more details in Methods). Relatedness between pair mates (Wang's relatedness coefficient $r$ ) is specified for each sampled pair next to the group number. Solid lines connect individuals with Wang's $r>0.487$ (mean $r$ for simulated parent-offspring dyads), dashed lines connect individuals with Wang's $r>0.247$ (mean $r$ for simulated half-offspring dyads), individuals with lower $r$ are not connected. The map was created in ArcGIS Desktop 10.6 (ESRI) and modified with Inkscape 1.0.1 (https://inkscape.org/).

In our study population, dispersal was most likely not sex-biased. This was indicated by the absence of spatial genetic structure in either sex and the lack of obvious geographic clustering in the mtDNA haplotype network (Fig. 2, 3). We found no evidence that dispersal distances differed between sexes, as both mtDNA haplotype diversity and mean relatedness were similar in females and males, suggesting that both sexes migrated opportunistically over varying distances. 
As geographic scale of our study was confined, with the maximum distance between homerange centers of only $3200 \mathrm{~m}$, these results should be treated as preliminary. However, they are in line with the direct observation of dispersal and the kin structure of the study population. In one directly observed case of dispersal, a subadult male (the oldest offspring of Group 1), moved to an unoccupied area adjacent to his natal group and later formed a pair (Group 11) with an unknown female (see Supplementary Materials for more details). The female did not have any close relatives among the sampled animals, indicating that she, unlike her mate, had not dispersed from any of the neighboring groups. The closest relative of this female was the adult male of Group 4 with $r=0.156$ (corresponding to a relatedness level between unrelated and half-sibling), with whom she also shared the same mtDNA haplotype (Supplementary Table S1). Patterns of relatedness between sampled adults (Fig. 1) further suggested that while some dispersers stay in the area (indicated by closely related individuals occupying home ranges that are either adjacent or separated by 1-2 home ranges), others migrate further (as many individuals in the study area have low relatedness). Overall, the absence of evidence for sex bias in dispersal is consistent with the theoretical expectations for the pair-living territorial species, where sexes should experience similar competition for mates and similarly low chances of breeding in acquiring breeding positions in their natal groups (Dobson 1982).

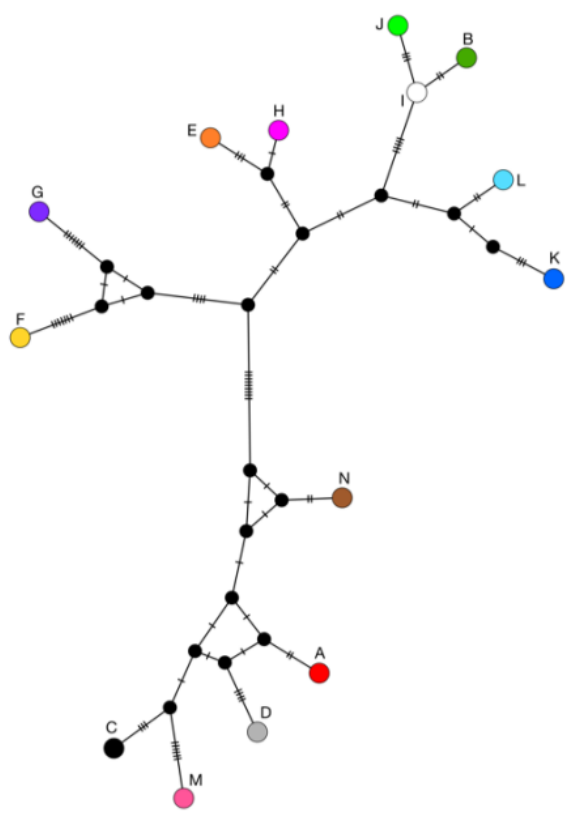

Fig. 3. A median joining network of all mtDNA haplotypes found in our study groups, constructed in PopART (Leigh \& Bryant 2015). The number of hatch marks indicates the number of mutations. Black nodes indicate inferred median vectors. The colors representing mtDNA haplotypes match those used in Fig. 1, 2. 
Our findings on dispersal and mating patterns, although preliminary, are in line with the evidence from other studies indicating that even opportunistic, not sex-biased dispersal can be sufficient to prevent inbreeding, as long as it is unconstrained by habitat fragmentation or other factors. The importance of unconstrained dispersal for inbreeding avoidance was supported in a simulation study based on empirical dataset from golden-crowned sifakas, Propithecus tattersalli, showing that high levels of outbreeding can be maintained in a population by a combination of social structure and unconstrained dispersal but without the need for active inbreeding avoidance mechanisms (Parreira et al. 2020). The link between dispersal and inbreeding risk was further indicated by studies demonstrating a correlation between dispersal distances and inbreeding level (e.g., in great tits, Parus major (Szulkin \& Sheldon 2008)). At our study site, the habitat was undisturbed, and dispersal was most likely unconstrained, ensuring passive inbreeding avoidance. As indicated by one case where pair mates were related on the level of second-degree kin, occasional inbreeding can still occur in such populations and is presumably tolerated.

In addition to dispersal and preferential mating with unrelated individuals, another way to avoid inbreeding is through engaging in EPC. Positive relationship between EPP rates and pair mate relatedness was demonstrated in many bird species (Jennions \& Petrie 2000; Foerster et al. 2003; Arct et al. 2015). However, pair-living mammals do not seem to use this strategy often, possibly because mammals are less mobile than birds and it might be harder for them to quickly evade their social mate and sneak EPC. In pair-living mammals, a similar strategy was, to our knowledge, only demonstrated in one species, fat-tailed dwarf lemur, where females sharing more MHC-supertypes with their social partner were shown to engaged in more EPC (Schwensow et al. 2008). In our study population, the absence of evidence for EPP further confirms our suggestion that dispersal in this habitat is unconstrained and the potential for inbreeding is low, rendering EPC unnecessary.

Summing up, the current study is the first to examine the link between mating system, mate choice and dispersal in a wild population of a pair-living primate. We showed that coppery titis in our study population are mostly genetically monogamous. This is likely due to a strong pair bond enabling effective mate guarding and relatively low population density limiting the opportunities for extra-pair copulations. We further showed that coppery titis, despite exhibiting no active inbreeding avoidance via mate choice, still had low relatedness between pair mates. Our results suggest that even opportunistic dispersal, as long as it is not constrained, can create sufficient genetic dissimilarity between opposite sexes to render active mate choice and extra-pair copulations less necessary. Alternatively, the absence of 
relatedness-based mate choice could be a result of constraints on mate choice, where individuals have so few available mates that they cannot afford to be too selective.

Future studies with larger sample size will be needed to examine the extent of genetic monogamy in this study population, as well as other populations of coppery titis and to further investigate dispersal patterns. In particular, to examine if titis indeed lack the mechanisms for active inbreeding avoidance via kin discrimination, it will be necessary to compare mating patterns and levels of inbreeding in undisturbed vs. fragmented habitats. Additionally, the absence of relatedness- and heterozygosity-based mate choice in our study population, of course, does not mean that mate choice does not occur in titis at any level. To better understand mating patterns in titis, future studies will have to examine if mate choice is based on other factors, such as, e.g., variation in MHC loci, body condition or the size or quality of the territory. Finally, current data do not allow to conclude whether extra-pair paternity is absent (or rare) in this study population because extra-pair copulations are not advantageous to individuals or because socio-ecological constrains prevent them from engaging in extra-pair copulations. To address this question, future studies on larger samples would need to compare genetic quality and fitness of group vs. extra-pair offspring (should there be any) in this or other population of titi monkeys. 
CHAPTER 4: WHAT MAKES A PAIR BOND IN A NEOTROPICAL PRIMATE: FEMALE AND MALE CONTRIBUTIONS

Sofya Dolotovskaya ${ }^{1,2}$, Sarah Walker ${ }^{3}$, Eckhard W. Heymann' ${ }^{1}$

${ }^{1}$ Behavioral Ecology and Sociobiology Unit, German Primate Center, Göttingen, Germany

2 Primate Genetics Laboratory, German Primate Center, Göttingen, Germany

${ }^{3}$ Estación Biológica Quebrada Blanco, Quebrada Blanco, Río Tahuayo, Peru

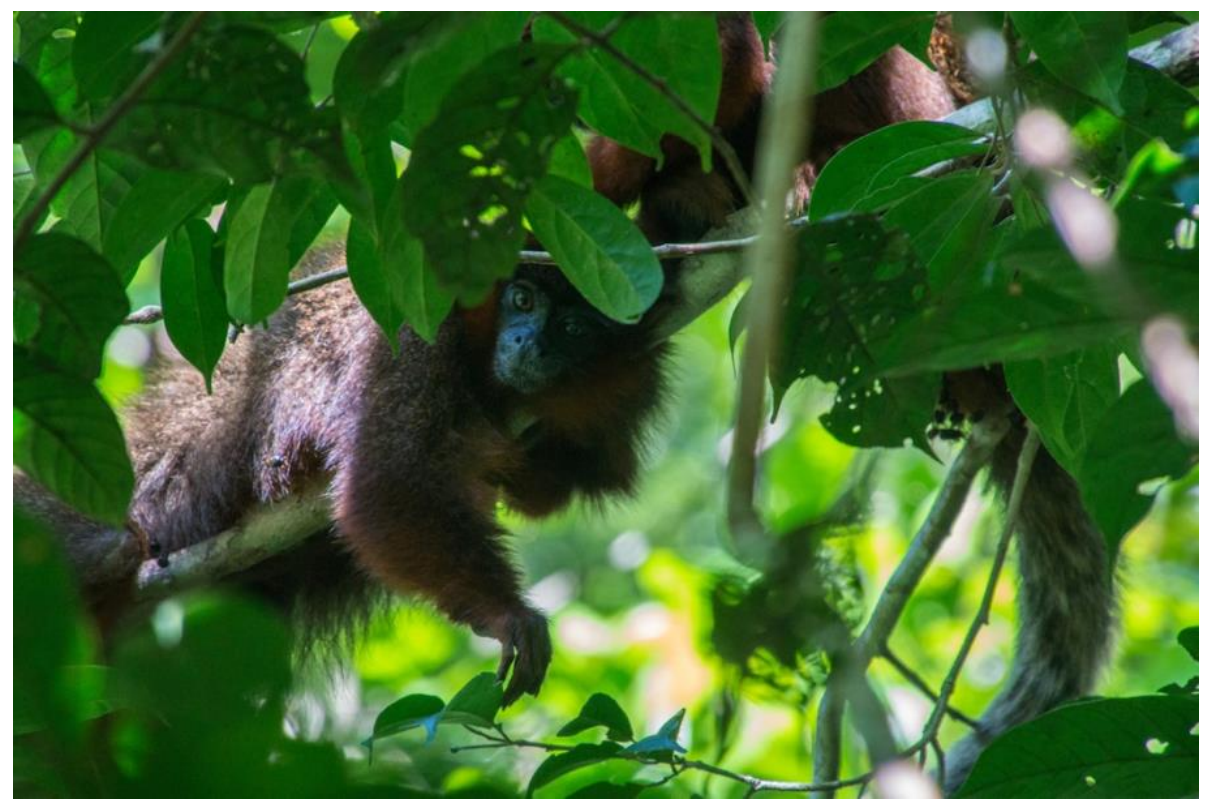

2019

Royal Society Open Science

http://dx.doi.org/10.1098/rsos.191489

(Accepted: 18 December 2019) 


\begin{abstract}
Pair living and pair bonding are rare in mammals, and the mechanisms of their maintenance remain a puzzle. Titi monkeys, a "textbook example" for "monogamous" primates, have strong pair bonds and extensive male care. To investigate mechanisms of pair-bond maintenance, we studied seven wild groups of red titis (Plecturocebus cupreus) in Peruvian Amazonia over a period of 14 months. We analysed pair bonds by measuring proximity, grooming, and approaches/leaves within pairs and collected data on intergroup encounters. Females contributed to grooming more than males, especially during infant dependency, when most of the grooming within pairs was done by females. Females were also more active in controlling proximity between pair mates, making most of the approaches and leaves. Males, on the other hand, invested more in territorial defenses. They participated in more intergroup encounters than females and were more active during these encounters. Our data is most consistent with the "male-services" hypothesis for pair-bond maintenance, where a female contributes more to the proximity and affiliation maintenance while a male provides beneficial services.
\end{abstract}

\title{
Introduction
}

Pair living, or social monogamy, is rare in mammals (3-9\%: Kleiman 1977; Lukas \& CluttonBrock 2013) and still remains an evolutionary puzzle. In contrast to birds, where pair living and biparental care are very common (90\%: Lack 1968), gestation and lactation in mammals restricts offspring nourishment to females, resulting in a highly skewed parental investment. Males are thus expected to increase their reproductive success through mating with multiple females rather than increasing their parental investment and remaining with a single female (Orians 1969; Trivers 1972).

Another mystery is why in some pair-living species adult males and females form pair bonds. Although the terms "pair bonding" and "pair living" are often used interchangeably, here we will consider them as separate components of a social system (Kappeler \& van Schaik 2002; Tecot et al. 2016). We define pair living as a type of social organization where two oppositesex adults share a home range or territory ("two-adult groups": Gowaty 1996; Kappeler \& van Schaik 2002; Fuentes 1999), and pair bonding as a type of social structure where adult male and female form a long-term (i.e., extending beyond one breeding season) affiliative relationship (Hinde 1983; Fuentes 2002). Pair living does not imply pair bonding but is often associated with it.

Pair bonds can be difficult to quantify for two reasons. First, pair-bond strength varies a lot between pair-living species (van Schaik \& Kappeler 2003). Some species form "dispersed" pairs: a male and a female share a common territory, but do not often interact and forage or 
sleep independently (e.g., maned wolves, Chrysocyon brachyurus: Dietz 2011; red-tailed sportive lemurs, Lepilemur ruficaudatus: Hilgartner et al. 2012; fork-marked lemurs, Phaner furcifer: Schülke \& Kappeler 2003). In other species, a male and a female are almost permanently associated (e.g., Kirk's dik-dik, Madoqua kirkii: Brotherton et al. 1997; Azara's night monkey, Aotus azarae: Huck et al. 2014). Second, in species forming two-adult groups, in contrast to multimale-multifemale groups with identifiable heterosexual dyads, pair bonding can be confounded with pair living (Fuentes 2002). To quantify pair bonds and tease it apart from pair living, a set of "diagnostic criteria" has been proposed: spatial relationship between pair mates, partner-specific behaviours and signs of distress during separation from the pair mate (Anzenberger 1992). These behaviours, in turn, can be assessed by rates of affiliative interaction, proximity scores, and measures of reciprocity between pair mates (Fuentes 2000).

Yet another difficulty with quantifying pair bonds is that the exact set of behaviours included in the concept of pair bond can depend on the definition used. In a narrow sense, often used in the zoological literature, the pair bond is assessed by rates and the degree of symmetry of proximity and affiliation between pair mates (Hinde 1983; Fuentes 2002, 2000). When used in a broader sense, pair bond can also include territorial behaviours such as mate guarding or assistance in resource defense or infant care (Fuentes 2002). It is not easy to disentangle different functions of territorial behaviours, some of which (e.g., mate guarding) might be related to the pair-bond maintenance while others (e.g., interest in extra-pair mates) might not. Moreover, neither territorial behaviours nor allomaternal care imply the existence of pair bonds, since both can be present in species without pair bonding or pair living (e.g., mate guarding in red-tailed sportive lemurs living in "dispersed" pairs: Hilgartner et al. 2012) or male care in group-living Barbary macaques, Macaca sylvanus (Small 1990). However, in the literature on pair bonds it is quite common to include all these behaviours in the set of pairbond maintenance behaviours, especially when they occur in already established pairs (e.g., Small 1990; Fernandez-Duque et al. 1997).

There are many hypotheses to explain the evolution and maintenance of pair living and/or pair bonding in mammals (see, e.g., Opie et al. 2013; Lukas \& Clutton-Brock 2013; Klug 2018; Tecot et al. 2016; Fuentes 2002). Here we discuss these hypotheses with the regard to the pair bonding and focus on the explanations they suggest for its maintenance, rather than the evolutionary origins. We differentiate these hypotheses according to whether pair bonding provides benefits to only one or to both sexes and outline predictions they make regarding the female and male contributions to the pair bond. 
(1) The "resource-defense hypothesis": both a male and a female benefit from pair bonding to defend resources together (van Schaik \& Dunbar 1990). Under this hypothesis, a male and a female are expected to be equally interested both in maintaining proximity and affiliation with a pair mate and defending their territory.

(2) The "mate-defense hypothesis": bonding with a female is beneficial for a male when either the spatial distribution of females or the temporal distribution of fertile periods make it difficult for the males to defend access to more than one female at a time (Emlen \& Oring 1977). This hypothesis suggests that a male should be more interested in maintaining proximity and affiliation with a pair mate. Both sexes can contribute to the territorial defense, but for different reasons: while a male is expected to defend exclusive access to a female, a female can defend resources.

(3) The "male-services hypothesis": a female benefits from bonding with a male when the male provides some important services such as territorial or antipredator defense, infant care or protection from infanticide by competing males (van Schaik \& Dunbar 1990; Palombit 2000; Opie et al. 2013). Under this scenario, a female is expected to be more interested in maintaining proximity and affiliation with a pair mate while a male is expected to provide some significant services. This hypothesis does not make any assumptions about the territorial defense: while a male can invest in territorial defense to protect resources or infants, a female can participate in territorial defense as a form of mate guarding or to protect resources.

Neotropical titi monkeys (previously Callicebus; split into Callicebus, Plecturocebus, and Cheracebus: Byrne et al. 2016) are an excellent model to study the mechanisms of pair-bond maintenance. A textbook example of a "monogamous" primate, titis form long-term pair bonds (at least up to 12 years, as shown in 12-year study of wild population of Plecturocebus discolor (previously Callicebus discolor), the longest dataset available so far; (Van Belle, Fernandez-Duque, et al. 2016; Fernandez-Duque et al. 2013; Mason 1966; Kinzey 1981). Titis live in groups comprising one reproductive pair and one to three offspring (Fernandez-Duque et al. 2013; Kinzey 1981; Kinzey \& Robinson 1983; Bicca-Marques \& Heymann 2013). Pair bonds between adult males and females exhibit all "diagnostic" characteristics mentioned above: spatial cohesiveness between pair mates, partner-specific behaviours (male-female duets), signs of distress during separation and strong preference for pair mates over strangers of either sex (Anzenberger 1988; Mason 1975; Mendoza \& Mason 1986). Adult male titis contribute heavily to infant care: the infant is carried almost exclusively by the social father and is returned to the mother only to suckle; males also play with offspring and share 
food with them more often than females (Wright 1984; Kinzey \& Wright 1982; SpenceAizenberg et al. 2016; Kinzey 1981).

The goal of our study was to examine the mechanisms of pair-bond maintenance in titis. Specifically, we wanted to assess (1) which factors affect rates of proximity and affiliation between pair mates; (2) which sex contributes more to the proximity and affiliation maintenance; (3) which sex contributes more to the territorial defense. We examined grooming and proximity patterns within pairs and collected data on male and female participation in territorial defense in seven wild groups of red titis (Plecturocebus cupreus) in the Peruvian Amazon. We compare our results to the data from other pair-bonded mammals and discuss our findings in the broader context of evolution and maintenance of pair-bonding in mammals.

\section{Methods}

\section{Study site and animals}

The study was conducted at the Estación Biológica Quebrada Blanco (EBQB) in the northeastern Peruvian Amazon ( $\left.4^{\circ} 21^{\prime} \mathrm{S}^{\circ} 3^{\circ} 09^{\prime} \mathrm{W}\right)$. We studied seven habituated titi groups in JuneDecember 2017 and 2018. Group 1 had been habituated since 1997; the other groups were habituated during this study. On average, it took 6 (3-10) weeks to habituate a group. We began data collection only after the animals were fully habituated. We individually identified all the study animals based on the combination of body size, tail shape and colouration, and genital size and shape. During the study period, infants were born in 5 groups. Birthdates and the composition of study groups is provided in the electronic supplementary material, table S1. We defined infant dependency as the period until an infant was not carried by a male during group travel (at the age of ca. 4.5 months: Fragaszy et al. 1982; Wright 1984). This also encompasses the period of most active lactation, as weaning begins when the infants are ca. 4.5 months old (Fragaszy et al. 1982).

\section{Data collection}

We followed each group in blocks of 5-6 days with the help of trained field assistants. In between periods of data collection, we monitored each group for 1-2 days a month for possible changes in group membership. We followed titis from the early morning when the animals left a sleeping site (or from when we located the group) until the late afternoon when the animals retired to a sleeping site (or until we lost them).

We used continuous focal animal sampling for the adult male and female of each group. We separated the focal samples on any given animal either by a focal sample of another animal or by, at least, a 10-min period. As focal animals were visible for variable periods of time, 
sampling periods varied from 3 min to $2 \mathrm{~h}$. If the focal animal was out of view for more than $2 \mathrm{~min}$, we terminated the observation. We discarded any samples where the focal animal was visible for less than $50 \%$ of time. We recorded social interactions (resting in body contact, active/passive grooming, and duetting; based on ethograms provided by Kinzey, 1981), the distance, and events of approaches and leaves (within $1 \mathrm{~m}$ ) between pair mates.

We also recorded intergroup encounters scored when individuals of the study groups had visual contact with another group and responded to its presence by calling and/or chasing (in the wild, titis very rarely engage in direct physical attacks or fighting during the encounters, even though this has been occasionally observed in captivity: Wright 1984, 2013; Robinson 1979; Mason 1966). We considered two encounters to be independent when all participants stopped calling and chasing for more than $30 \mathrm{~min}$. We recorded the time, location, identities of participating groups and individuals, and the activities of participants (calling, chasing). Participation was scored when an individual was either calling, chasing, or both. If in the beginning of an intergroup encounter an individual called alone and/or moved alone towards another group, we scored this individual as the initiator of the encounter.

\section{Statistical analyses}

To characterize rates of proximity and affiliation between pair mates, we calculated daily proportions of time pair mates spent in close proximity $(\leq 1 \mathrm{~m})$, including time spent in affiliative interactions (resting and in body contact and grooming) for each pair.

\section{Factors affecting rates of affiliation and proximity between pair mates}

To examine which factors affect rates of proximity and affiliation between pair mates, we used a Generalized Linear Mixed Model (GLMM) (Baayen 2008) with beta error structure and logit link function. We used the presence of dependent infant (hereafter "infant presence"), group size, and season as fixed effects and group ID as a random effect. As a measure of seasonality, we used rainfall data (monthly averages in mm at Tamshiyacu $\left(4^{\circ} 00^{\prime} 10.7^{\prime \prime} \mathrm{S} 73^{\circ} 09^{\prime} 38.2^{\prime \prime} \mathrm{W}\right)$, ca. $40 \mathrm{~km}$ from EBQB, available at https://www.worldweatheronline.com). We compressed the response to avoid zeros and ones using $y^{\prime}=\left(y^{*}(n-1)+0.5\right) / n$, where $n$ is the sample size (Smithson \& Verkuilen 2006). To achieve an approximately symmetrical distribution, we further square root-transformed the response. We z-transformed group size and rainfall (Schielzeth 2010). To reduce the probability of Type I Error (Barr et al. 2013) we included the random slope of rainfall within group and its correlation with the intercept. We tested the overall effect of infant presence, group size, and season using a full-null model comparison based on a likelihood ratio test (Dobson et al. 2008; Forstmeier \& Schielzeth 2011). The null model lacked the fixed effects but was otherwise identical to the full model. We tested the fixed effects using likelihood ratio tests comparing the full model with reduced models 
excluding each of the effects one at a time (Barr et al. 2013). To assess model stability, we compared the full model estimates with those obtained from models with the levels of the random effects excluded one at a time. The sample had 269 daily proportion values, taken from 7 pairs.

\section{Grooming reciprocity}

To assess grooming reciprocity between pair mates, we first calculated the grooming reciprocity index for each pair (Nishida 1988):

$$
\frac{G f m-G m f}{G f m+G m f}
$$

where $\mathrm{Gfm}$ is the amount of time that the female groomed the male and $\mathrm{Gmf}$ is the amount of time that the male groomed the female. The index ranges from -1 to 1 ; values closer to 1 indicate that a female grooms a male more than vice versa.

To further examine if grooming reciprocity is affected by infant presence, we used a GLMM with daily proportion of time a female groomed a male of the total grooming time between pair mates as a response. We compressed the response using the formula provided above (Smithson \& Verkuilen 2006). The model design, including predictor transformations, was identical to that of the model described above, except for the correlation between the random slope and the random intercept being unidentifiable (as indicated by absolute values of 1 ) and thus excluded from the model. The null model used for full-null model comparison lacked the effect of infant presence. The sample had 103 daily proportion values, taken from 7 pairs. Both models were fitted in $\mathrm{R}$ (version 3.5.3; R Core Team 2018) using the package glmmTMB (version 0.2.3; Brooks et al. 2017). To check for collinearity between predictors, we determined Variance Inflation Factors (Quinn \& Keough 2002) with the function vif of the package car (version 3.0.2; Fox \& Weisberg 2011). To assess model stability, we used a function kindly provided by Roger Mundry.

\section{Proximity maintenance}

To assess which individual was more responsible for maintaining proximity between pair mates, we first calculated the Hinde index (Hinde \& Atkinson 1970):

$$
100 *\left(\frac{A f}{A f+A m}-\frac{L f}{L f+L m}\right)
$$

where $A f$ is the number of female approaches, $A m$ is the number of male approaches, $L f$ is the number of female leaves, and $\mathrm{Lm}$ is the number of male leaves. The index ranges from -100 to +100 ; high values indicate that proximity is mainly maintained by a female. 
However, values of the Hinde index are difficult to interpret, since they do not indicate which individual makes most approaches and leaves, and different proximity patterns can thus result in the same values (a value of 0 can occur because female made equal number of approaches and leaves or because male made all approaches and leaves). To assess which individual is more active in maintaining proximity (makes more approaches and leaves), we calculated the Brown's index (Brown 2001) using the same arguments:

$$
100 * \frac{A f+L f}{A f+A m+L f+L m}
$$

The index ranges from 0 to 100; high values indicate that a female makes most approaches and leaves.

\section{Results}

Factors affecting rates of proximity and affiliation between pair mates

Infant presence and group size had a clear impact on rates of proximity and affiliation between pair mates (full-null model comparison $\chi 2=18.348, \mathrm{df}=3, \mathrm{P}<0.001$ ). Specifically, pair mates spent less time in close proximity after infant birth $(\chi 2=16.524, \mathrm{df}=1, \mathrm{P}<0.001)$, and in larger groups pair mates spent less time in proximity than in smaller groups, although this effect was borderline significant $(\chi 2=3.759, \mathrm{df}=1, \mathrm{P}=0.053)$. Rainfall had no significant effect $(\chi 2=0.266, \mathrm{df}=1, \mathrm{P}=0.610)$ (supplementary material table S2; fig. 1).

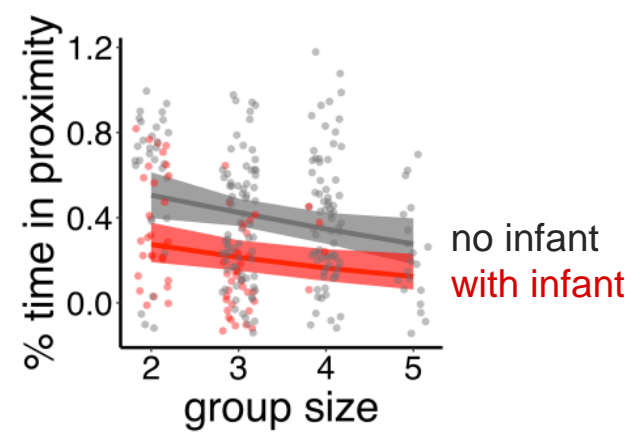

Fig 1. Daily proportion of time pair mates spent in close proximity as a function of group size, separately for the absence and presence of infant. The lines depict the fitted model (based on rainfall at its average), and grey and red areas show corresponding $95 \%$ confidence intervals.

\section{Grooming reciprocity}

Overall, females groomed males more than vice versa, as indicated by the values of the grooming index closer to 1 (table 1). Grooming reciprocity between pair mates was further affected by infant presence (fig. 2). While grooming was almost reciprocal before infant birth, females groomed males more than vice versa after infant birth (GLMM; likelihood ratio test comparing full and null model: $\chi 2=15.403, \mathrm{df}=1, \mathrm{P}<0.001)$. 
(a)

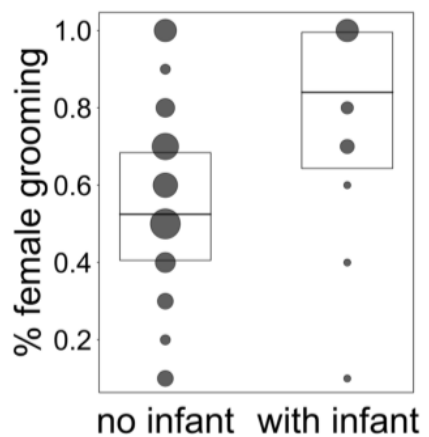

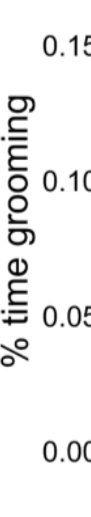

(b)

female grooms male

male grooms female

Fig. 2. (a) Proportions of female investment in grooming within pairs, before vs. after infant birth. For visual clarity proportion was binned into 10 sections. The area of the dots corresponds to the respective number of observations (0 to 18 per bin). Boxes depict median and lower and upper quartiles. (b) Mean daily proportion of time spent grooming within pairs, before vs. after infant birth.

\section{Proximity maintenance}

Females were more active in maintaining proximity, making the majority of both approaches and leaves within pairs as indicated by Brown's index and proportion of female approaches (table 1). The values of Hinde index provided mixed results, indicating females to be more responsible for maintaining proximity in some pairs and males to be more responsible in other pairs; overall, however, the values were not substantially different from 0 (on a scale from -100 to +100 ). To exclude the possibility that primarily female activity in maintaining proximity was caused by a lactating female addressing an infant carried by a male and not the male itself, we further calculated Brown's index separately for periods with and without dependent infants; values for both periods were still higher than 50.0, indicating that females were more active in the relationships regardless the infant presence.

Table 1. Grooming, Hinde and Brown's indexes and proportion of female approaches from the total number of approaches within pairs.

\begin{tabular}{|l|l|l|l|l|}
\hline Group & $\begin{array}{l}\text { Grooming } \\
\text { index }\end{array}$ & Hinde index & Brown's index ${ }^{\mathbf{1}}$ & $\begin{array}{l}\text { Proportion of female } \\
\text { approaches }\end{array}$ \\
\hline 1 & 0.92 & -16.78 & $62.59(76.90 / 59.29)$ & 0.56 \\
\hline 2 & 0.96 & -20.84 & $60.91(50.85 / 72.55)$ & 0.54 \\
\hline 3 & 0.71 & -30.00 & $63.64\left(63.64 /{ }^{2}\right)$ & 0.50 \\
\hline 4 & 0.74 & 14.10 & $68.46^{3}$ & 0.74 \\
\hline 5 & 0.99 & 8.97 & $65.91^{3}$ & 0.69 \\
\hline 6 & 0.99 & 6.67 & $64.29(100.00 / 58.33)$ & 0.67 \\
\hline 7 & 0.76 & -4.17 & $64.71(64.29 / 66.67)$ & 0.63 \\
\hline Mean & $\mathbf{0 . 8 7}$ & $-\mathbf{6 . 0 1}$ & $\mathbf{6 4 . 3 6}$ & $\mathbf{0 . 6 2}$ \\
\hline
\end{tabular}

${ }^{1}$ Overall index with separate values for the periods with and without dependent infants, respectively, in parenthesis

2 Insufficient data to calculate the index

${ }^{3}$ Groups only observed in the absence of dependent infants 


\section{Intergroup encounters}

Of 21 observed intergroup encounters, 9 were initiated by a male and 12 did not have a clear initiator (for the full account of the encounters see the supplementary material, table S3). We never observed a female initiating an encounter. Males participated in all encounters, while females participated in 19 encounters. Males were more active during the encounters: in all 16 encounters for which the chasing data could be collected, males were both calling and chasing. In contrast, females mainly just called (16 encounters) and only chased during 2 encounters. We never observed a female chasing unless her mate was chasing, too.

\section{Discussion}

Overall, rates of proximity and affiliation between pair mates in red titi monkeys were affected by the presence of dependent infants and group size. After infant birth, pair mates spent less time in close proximity. A similar effect of infant presence was also demonstrated in a field study on P. discolor (Spence-Aizenberg et al. 2016). The decrease in time pair mates spend in proximity after infant birth is likely related to energetic costs of infant care that are high both for lactating females and carrying males (Altmann \& Samuels 1992) or to the fact that males, while spending substantial amount of time socializing with infants (Wright 1984; Kinzey \& Wright 1982; Spence-Aizenberg et al. 2016; Kinzey 1981), have less time available for their pair mates. Similarly, in larger family groups, i.e. those including juvenile and subadult offspring, with more potential social partners pair mates spent slightly less time in proximity than in smaller groups.

Females and males contributed differently to the pair bond maintenance. Females contributed more than males to proximity and affiliation maintenance. First, they groomed males more than vice versa, especially during the period of infant dependency. Second, females were more active in controlling proximity, making the majority of approaches and leaves within pairs as indicated by Brown's index and proportion of female approaches, suggesting more female initiative and "interest" in proximity. Males, on the other hand, contributed more than females to the territorial defense: they participated in more intergroup encounters and were more active during these encounters.

Primarily female contribution to the proximity and affiliation maintenance was demonstrated in most of the field studies on other titi species: in Plecturocebus toppini (previously Callicebus brunneus, Lawrence 2007), Cheracebus torquatus (Kinzey \& Wright 1982), and P. discolor (Porter 2016), females groomed males more than the reverse (although in one study on $P$. discolor grooming was reciprocal: Spence-Aizenberg et al. 2016). In captive Plecturocebus cupreus (previously Callicebus moloch), females were more attached to males than vice versa: they spent more time than males close to experimental partitions physically separating pair 
mates (Anzenberger 1988), preferred a pair mate to an empty cage or a stranger male more often than males did (Cubicciotti \& Mason 1978), and were more reluctant than males to interact with opposite-sex strangers (Fernandez-Duque et al. 1997). Approach rates and the Hinde index provided mixed results in titis: while in our study females approached males more often in all pairs, it was true only for some pairs in P. toppini (Lawrence 2007), and males approached more often in P. discolor (Fernandez-Duque et al. 2013). The Hinde index indicated neither sex to be more responsible for maintaining proximity in our study and in $P$. toppini (Lawrence 2007), but showed males to be more responsible in P. discolor (although the bias was not very pronounced, as indicated by low index values: 18 and 25, respectively: Lawrence 2007; Spence-Aizenberg et al. 2016). Unfortunately, none of these studies calculated the Brown index, and the difficulty of interpreting the values of Hinde index (see Methods) does not allow to assess which sex was more active in the relationship.

Primarily male contribution to the territorial defense is consistent with other titi studies. In P. discolor and P. toppini, males participated in more intergroup encounters than females, initiated them more often and called and chased more during the encounters (Lawrence 2007; Robinson 1981; Wright 1984, 2013). In P. discolor, males initiated the duetting near group boundaries more often than females, and responded stronger (i.e., initiated response duetting more often) than females to the simulated duets in playback experiments, indicating more active male involvement in the boundary reinforcement (Robinson 1981). In captive Plecturocebus cupreus (previously Callicebus moloch), males show more agitation and distress than females in the presence of intruders of both sexes (Mendoza \& Mason 1986; FernandezDuque et al. 1997; Cubicciotti \& Mason 1978; Fernandez-Duque et al. 2000).

Our observations, together with data on other titi species, are most consistent with the "maleservices" hypothesis that predicts that a female would show more initiative and "interest" in maintaining proximity and affiliation with a pair mate in exchange for some important services provided by a male. This hypothesis is further supported by a fact that grooming between partners was more heavily skewed towards female investment during the period of infant dependency, when male services are most needed. While males reduced the amount of grooming directed at females after infant birth, females conserved the amount of time they groomed males, suggesting the importance of maintaining proximity and affiliation with pair mates for the females. So which services does a male provide?

First, male titis provide extensive infant care, releasing the lactating females of all the costs of infant carrying, sharing food and socializing with them. Second, they provide anti-predator defense: both in our study groups (Dolotovskaya, Flores Amasifuen, et al. 2019) and in $P$. discolor , males were more active during encounters with predators. Although sex differences 
in vigilance have not been quantified for titis yet, both in our study (unpublished data) and in P. toppini (Lawrence 2007) males appeared to be more vigilant than females. By providing anti-predator defense, a male allows a female to focus on foraging (van Schaik \& Dunbar 1990). Interestingly, in P. discolor males demonstrated active anti-predator behaviours only in the presence of infants (De Luna et al. 2010).

Finally, males provide territorial defense. The function of this behaviour is likely mixed and can represent resource defense, mate defense, or both. Playback studies trying to tease apart these two functions of territorial defense provided somewhat more support for resource defense. In P. toppini, males reacted stronger to playbacks in the high-used versus low-used parts of the home range (Lawrence 2007). In P. discolor (Robinson 1981) and Callicebus nigrifrons (De Luna et al. 2010), males did not react stronger to playbacks of male solos than to playbacks of duets. Finally, in C. nigrifrons, pairs were not duetting more often during the periods of likely female fertility (Caselli et al.2014). Mate defense received only weak support in P. toppini: males reacted stronger when duets were played closer to their mates (Lawrence 2007). In captive P. cupreus, however, males clearly demonstrated mate-guarding behaviour: they showed increased attraction to a pair mate and agonism towards a male intruder as a function of increasing proximity between the pair mate and the intruder (Cubicciotti \& Mason 1978; Fernandez-Duque et al. 2000).

It is likely that participation in the intergroup encounters serves both for resource and mate defense, as these functions are not mutually exclusive. Territorial defense ensures exclusive use of space, which in turn allows exclusive access to both resources and mates (Hall 2004). In this respect, it should be noted that females participated in most (19 of 21) intergroup encounters together with males, even if they were not as active as males and, unlike males, almost never chased the animals from the neighbouring group. Female participation in encounters provides some support for the "resource-defense" hypothesis where both sexes defend their territory together. However, more active male participation in territorial defense together with more pronounced female contribution to the proximity and affiliation maintenance provide arguments in favour of the "male-services" hypothesis.

Another likely reason for the males to participate in the intergroup encounters represents the other side of the mate defense: an interest in extra-pair mates. This possibility cannot be ruled out neither for males nor females. There is one report on extra-pair copulations in titis (Mason 1966) and several reports on mate displacements (Lawrence 2007; Bossuyt 2002). In the field (Lawrence 2007; Robinson 1981) and captive (Anzenberger 1988; Cubicciotti \& Mason 1978) studies, both sexes demonstrated mate-guarding behavior (e.g., responded stronger to the same-sex playback calls than to opposite-sex calls), although males to a greater extent. Pair 
mates were also more affiliative during the intergroup encounters in P. toppini and P. discolor (Lawrence 2007; Robinson 1981), a behavior likely enabling both sexes to guard their partners from potential extra-pair mates.

Comparison with other pair-bonded mammals suggests an association between the intensity of male care for infants and the pattern on pair-bond maintenance (table 2). Generally, the more intense male care is, the more a female contributes to the maintenance of proximity and affiliation with a male. While in species with no male care males are primarily responsible for proximity and affiliation maintenance, in species with moderate or intense male care females contribute to proximity and affiliation maintenance equally or more than males. The only exception is sakis, where females contribute to proximity and affiliation maintenance more than males despite the complete absence of male care. However, it has been shown that male sakis contribute more than females to territorial and anti-predator defense, especially during the infant dependency (Thompson \& Norconk 2011; De Luna et al. 2010), possibly providing indirect benefits to females. Interestingly, like in our study, the skew towards female contribution to proximity and affiliation maintenance was more pronounced during the period of infant dependency (Thompson \& Norconk 2011). This might indicate a female's increased value of male services during the period when these services are most needed. Male care has been suggested as a driver for the evolution of pair living and pair bonding (Kleiman 1977; Lack 1968). And although recent phylogenetic analyses across mammals suggest that male care is more likely a consequence of pair living than a cause (Lukas \& Clutton-Brock 2013; Opie et al. 2013), it seems to be an important factor affecting the mechanisms of pairbond maintenance.

In sum, our study demonstrates that in red titi monkeys, females contribute more to proximity and affiliation maintenance, while males contribute more to territorial defense and infant care. Our data is most consistent with the "male-services" hypothesis for pair-bond maintenance, where a male provides services beneficial for a female, who, in turn, shows more initiative and 'interest' in maintaining proximity and affiliation with a male. To a lesser extent, our findings also provide some support for the "resource-defense" hypothesis, where both pair mates jointly defend their territory. Comparisons with other pair-bonded mammals suggest that male care might represent an important factor for the maintenance of pairbonds. 
Table 2. Intensity of male care and sex investment in the proximity and affiliation maintenance for pairbonded mammals based on data from field studies. Male care: $\mathrm{N}=$ no care, $\mathrm{M}=$ moderate care, $\mathrm{I}=$ intense care (following classification criteria in Huck et al. 2014).

\begin{tabular}{|c|c|c|c|c|}
\hline Species & $\begin{array}{l}\text { Male } \\
\text { care }\end{array}$ & $\begin{array}{l}\text { Which sex contributes } \\
\text { more to proximity and } \\
\text { affiliation maintenance }\end{array}$ & $\begin{array}{l}\text { Measures of } \\
\text { contributions used }\end{array}$ & References \\
\hline $\begin{array}{l}\text { Madoqua kirkii } \\
\text { (Kirk's dik-dik) }\end{array}$ & $\mathrm{N}$ & Males & Approach/leave data & $\begin{array}{l}\text { Brotherton et } \\
\text { al. } 1997\end{array}$ \\
\hline $\begin{array}{l}\text { Hylobates lar } \\
\text { (white-handed } \\
\text { gibbon) }\end{array}$ & $\mathrm{N}$ & Males & $\begin{array}{l}\text { Grooming reciprocity, } \\
\text { approach/leave data }\end{array}$ & $\begin{array}{l}\text { Palombit et al. } \\
1996\end{array}$ \\
\hline Indri indri & $\mathrm{N}$ & Males & Grooming reciprocity & Pollock 1979 \\
\hline $\begin{array}{l}\text { Pithecia pithecia } \\
\text { (white-faced saki } \\
\text { monkey) }\end{array}$ & $\mathrm{N}$ & Females & $\begin{array}{l}\text { Grooming reciprocity, } \\
\text { approach/leave data }\end{array}$ & $\begin{array}{l}\text { Thompson \& } \\
\text { Norconk 2011; } \\
\text { Fernandez- } \\
\text { Duque et al. } \\
2013\end{array}$ \\
\hline $\begin{array}{l}\text { Symphalangus } \\
\text { syndactylus (siamang) }\end{array}$ & M & Both sexes & $\begin{array}{l}\text { Grooming reciprocity, } \\
\text { approach/leave data }\end{array}$ & $\begin{array}{l}\text { Palombit et al. } \\
1996\end{array}$ \\
\hline $\begin{array}{l}\text { Petropseudes dahli } \\
\text { (rock-haunting } \\
\text { possum) }\end{array}$ & I & Both sexes & Approach data & Runcie 2000 \\
\hline $\begin{array}{l}\text { Otocyon megalotis } \\
\text { (bat-eared fox) }\end{array}$ & I & Both sexes & Approach data & $\begin{array}{l}\text { Wright 2006; } \\
\text { Wright et al. } \\
2010\end{array}$ \\
\hline $\begin{array}{l}\text { Aotus nancymaae } \\
\text { (owl monkey) }\end{array}$ & I & Both sexes ${ }^{a}$ & $\begin{array}{l}\text { Grooming reciprocity, } \\
\text { approach/leave data }\end{array}$ & $\begin{array}{l}\text { Wolovich et al. } \\
2017 ; \\
\text { Wolovich \& } \\
\text { Evans } 2007\end{array}$ \\
\hline $\begin{array}{l}\text { Plecturocebus cupreus } \\
\text { (red titi monkey) }\end{array}$ & I & Females & $\begin{array}{l}\text { Grooming reciprocity, } \\
\text { approach/leave data }\end{array}$ & This study \\
\hline
\end{tabular}

(a) Data available only for captive animals 


\section{CHAPTER 5: DO LESS OR EAT MORE: STRATEGIES TO COPE WITH COSTS OF PARENTAL CARE IN A PAIR-LIVING MONKEY}

\section{Sofya Dolotovskaya ${ }^{1,2}$, Eckhard W. Heymann ${ }^{1}$}

\footnotetext{
${ }^{1}$ Behavioural Ecology and Sociobiology Unit, German Primate Center, Göttingen, Germany

2 Primate Genetics Laboratory, German Primate Center, Göttingen, Germany
}

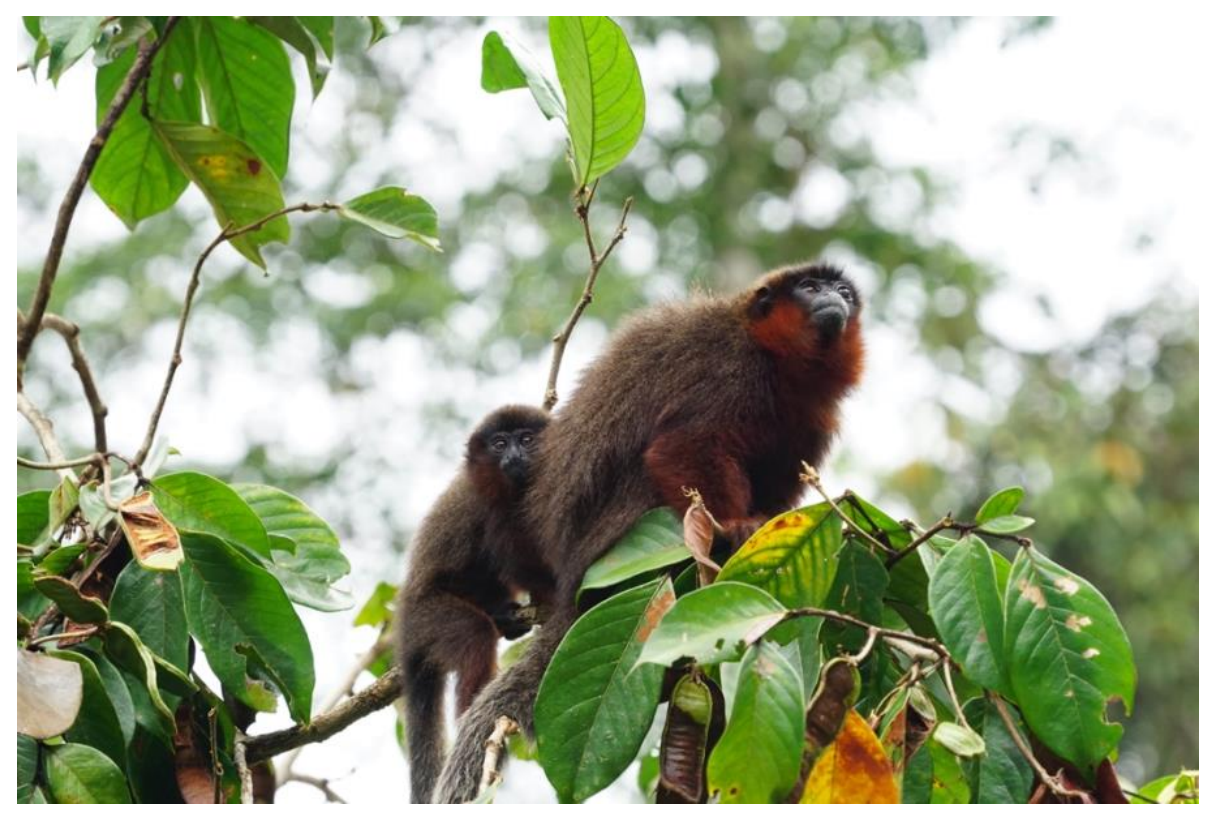

\section{Animal Behaviour}

https://doi.org/10.1016/j.anbehav.2020.03.012

(Accepted 25 February 2020) 


\section{Abstract}

Infant care is costly, and strategies to deal with its energetic demands may involve increasing feeding time or reducing activity levels or social time. In mammals, the most energetically expensive form of infant care is lactation, followed by infant carrying in species where young are transported over long distances. In titi monkeys, infants are carried primarily by males, which allows us to tease apart the effects of lactation and infant carrying. We analysed activity budgets and diet composition in adult males and females in seven free-ranging groups of red titi monkeys, Plecturocebus cupreus, in the Peruvian Amazon over 14 months. Females fed more, consumed more arthropods and rested less after infant birth than before, and males fed less, consumed fewer arthropods and rested more. Lactating females increased feeding time at the expense of mainly resting and, to a lesser degree, social time. Despite the general decrease in social time, females conserved grooming time within pairs. Our results suggest that females and males use opposing strategies to cope with the costs of parental care and indicate the importance of pair-bond maintenance for females.

\section{Introduction}

Infant care is costly and requires the caring adults to allocate time and energy at the expense of their own maintenance, survival or future mating opportunities (Maynard Smith 1977). In mammals, the most energetically costly investment in young is lactation (Altmann \& Samuels 1992; Clutton-Brock et al. 1989). While in most mammals infants are not transported over long distances during foraging or travelling, infant carrying is present in six eutherian mammalian orders (Primates, Chiroptera, Dermoptera, Xenarthra, Pholidota and Sirenia), and its energetic costs are second only to lactation, at least in primates (Altmann \& Samuels 1992).

Lactation has been thoroughly studied, and its direct energetic costs have been measured for many species (e.g. Butte \& King, 2005; Costa, Boeuf, Huntley, \& Ortiz, 1986; Sikes, 1995). In many mammals, females support the energy demands of lactation by increasing either feeding time (e.g. red deer, Cervus elaphus, Clutton-Brock, Iason, Albon, \& Guinness, 1982; Hanuman langur, Presbytis entellus, Koenig, Borries, Chalise, \& Winkler, 1997; hoary bat, Lasiurus cinereus, Barclay, 1989) or consumption of nutritionally valuable resources (squirrel monkey, Saimiri oerstedi, Boinski, 1988; red-ruffed lemur, Varecia rubra, Vasey, 2004). In some species, however, females do the reverse: instead of eating more, they reduce activity levels, thus minimizing their energetic needs, and presumably using their fat reserves. For example, in chacma baboons, Papio ursinus, and chimpanzees, Pan troglodytes, lactating females decrease feeding time and activity levels (Barrett et al. 2006; Murray et al. 2009). One possible reason for this is that some competing activity, for example vigilance, is incompatible 
with feeding. Indeed, in chacma baboons there is a negative correlation between feeding time and vigilance (Miller 2002; Barrett et al. 2006).

When using the energy-maximizing 'eat more' strategy, females must offset the increased feeding time by withdrawing time from other activities. The classical model by Altmann (1980) of maternal time budgets in primates suggests that extra feeding time could be drawn only from social time, since resting and moving time would be set by the demands of the habitat and activity of the group as a whole. While this assumption is consistent with Altmann's data on yellow baboons, Papio cynocephalus, in other Papio species, geladas, Theropithecus gelada, and black howlers, Alouatta pigra (Dias et al. 2011; Dunbar \& Sharman 1984; Dunbar \& Dunbar 1988), social time is conserved for as long as possible and extra feeding time is taken from resting time. Only when resting time is exhausted, do females give up social time. In a study of captive cotton-top tamarins, Saguinus oedipus, feeding time also increased mainly at the expense of resting time, and although active forms of socializing (allogrooming) decreased, time spent in contact with other group members did not change (Price 1992a). It has been suggested that surrendering social time has serious consequences for animals as social as primates (Dunbar \& Dunbar 1988).

Compared to lactation, relatively little is known about the energetic costs of infant carrying. In most species in which it occurs, infants are carried by lactating females (Kleiman \& Malcolm 1981; Woodroffe \& Vincent 1994). The females then have the double burden of lactation and infant carrying, which makes it difficult to disentangle their respective energetic costs. This topic is best addressed in primates, where both infant carrying and allomaternal care are relatively widespread (Huck \& Fernandez-Duque 2012b). The direct energetic costs of infant carrying have been analysed by measuring changes in body mass in several captive studies on cooperatively breeding cotton-top tamarins, where infants are transported by all group members. In these studies infant-carrying helpers were shown to lose considerable amounts of body mass, and this loss was correlated with the time spent carrying (Sánchez et al. 1999; Achenbach \& Snowdon 2002). Infant carrying was also shown to affect activity patterns in helping males both in cooperatively breeding callitrichids and in pair-living titi monkeys, and the direction of change suggested the use of the energy-minimizing 'do less' strategy. In wild saddleback, Saguinus fuscicollis (now Leontocebus weddelli), moustached, Saguinus mystax, and captive cotton-top tamarins, males fed less and rested more during carrying periods (Goldizen 1987; Price 1992b; Huck et al. 2004). In wild dusky titi monkeys, Callicebus brunneus (now Plecturocebus toppini), carrying males were less effective in catching arthropods (Wright 1984). A possible explanation might be again the conflict between foraging and vigilance. This effect has been suggested to be more pronounced in species using 
crypsis as a main antipredator strategy, as the need for infant-carriers to remain concealed will be incompatible with effective foraging (Tardif 1994, 1997), especially with manoeuvres required for prey capture.

Neotropical titi monkeys (previously Callicebus; now split into Callicebus, Plecturocebus and Cheracebus: Byrne et al., 2016) are a good model to study the effects of both lactation and infant carrying. Titi monkeys live in groups comprising one reproductive pair and one to three young (Fernandez-Duque et al. 2013; Kinzey 1981; Kinzey \& Robinson 1983; Bicca-Marques \& Heymann 2013) and have one of the highest levels of male care among primates. Both in the wild and in captivity, the infant is carried almost exclusively by the adult male from the first week of life; mothers contribute very little to carrying and mainly during the first days after birth and later the infant is returned to the mother only to suckle (Tirado Herrera \& Heymann 2004; Fernandez-Duque et al. 2013; Spence-Aizenberg et al. 2016; Lawrence 2007; Wright 1984; Fragaszy et al. 1982; Jantschke et al. 1995). This allows us to tease apart the effects of lactation and infant carrying. So far, only two studies have addressed this topic in Plecturocebus spp. (Tirado Herrera \& Heymann 2004; Wright 1984); they showed that lactating females increased their consumption of protein-rich food (arthropods), but were based on just one and two groups, respectively.

The goal of our study was to examine the effects of lactation and infant carrying in titi monkeys. Specifically, we aimed at investigating (1) how activity patterns change in lactating females and carrying males; (2) how diet composition changes in lactating females and carrying males; (3) if feeding time increases in lactating females, which activity category is compromised. To address these questions, we analysed activity budgets and diet composition of adult males and females from seven free-ranging groups of red titi monkeys, Plecturocebus cupreus, in the Peruvian Amazon.

\section{Methods}

\section{Study site and animals}

The study was conducted at the Estación Biológica Quebrada Blanco (EBQB) in the Peruvian Amazon $\left(4^{\circ} 21^{\prime} \mathrm{S}, 73^{\circ} 09^{\prime} \mathrm{W}\right)$. We studied seven adult female-male pairs from seven habituated titi groups in June-December 2017 and June-December 2018. Group 1 had been habituated to the presence of human observers and studied intermittently since 1997; the other groups were habituated during this study. On average, it took 6 (3-10) weeks to habituate a group. We began data collection only after the animals were fully habituated, allowing the observers to approach to within $5 \mathrm{~m}$ without fleeing or hiding. We individually identified all the study animals based on the combination of body size, tail shape and coloration and genital size and 
shape. We distinguished females and males based on genitalia shape and body size and shape (males are slightly bigger and thicker around the neck).

During the study period, infants were born in five groups. We defined infant dependency as the period until an infant was locomoting independently most of the time at the age of ca. 4.5 months, or 19 weeks (Fragaszy et al. 1982; Wright 1984; Jantschke et al. 1995). In captive titis, this age also marked the onset of weaning conflicts and the change in the form of infant contact with adults, with clinging being replaced by adult forms of contact such as sitting in contact, grooming and tail twining (Fragaszy et al. 1982). Lactation can be difficult to see in wild titis, and there is only one report on lactation duration in a wild group, where suckling was observed until an infant was 8 months old (Wright 1984). We did not quantify lactation in our study, but we observed sucking various times a day when infants were $<5$ months old and only once or twice a day when an infant was ca. 6.5 months old. Twice we also observed a 10-month-old infant suckling on a female's belly and shoulder, indicating the difficulty of assessing the duration of actual lactation. For this reason, we used a conservative estimate of high-intensity lactation, inferred from the duration of lactational anovulation in captive titis as ca. 6.5 months, or 28 weeks (Valeggia, Mendoza, Fernandez-Duque, Mason, \& Lasley, 1999). We estimated an infant's date of birth as the midpoint between the dates when a group was last seen without and first seen with an infant. The difference between these dates varied between 0 and 26 days. For Group 3, the date of birth could only be estimated within a month based on body size as the group already had an infant when we started to follow it. However, at the end of the study period the infant still did not begin to travel independently, suggesting that it was younger than 4 months. Birthdates, group composition and sampling effort for the study groups are provided in Table 1.

\section{Data collection}

We followed each group in blocks of 5-6 days with the help of trained field assistants. Focal data were collected by S.D. and two field assistants. New observers went through a period of training when they collected 'test' data in parallel with S.D. until an agreement was achieved between them. Focal samples collected during the first week of observation were excluded from the analysis. In between periods of data collection, we monitored each group for 1-2 days a month for possible changes in group membership. We followed titis from the early morning when the animals left a sleeping site (or from when we located the group) until the late afternoon when the animals retired to a sleeping site (or until we lost sight of them).

We used continuous focal animal sampling for the adult male and female of each group. We separated the focal samples on any given animal either by a focal sample of another animal or by, at least, a 10 min period. As focal animals were visible for variable periods of time, 
sampling periods varied from 3 min to $2 \mathrm{~h}$. If the focal animal was out of view for more than $2 \mathrm{~min}$, we terminated the observation. We discarded any samples where the focal animal was visible for less than $50 \%$ of the time. We used activity categories falling into four classes: resting, moving, feeding and socializing (resting in body contact, grooming, social playing) based on ethograms provided by Kinzey (1981) and Spence-Aizenberg et al. (2016). During feeding we specified the type of food as fruits, leaves, shoots, flowers, arthropods, soil (from termite nests) or unknown when the food item could not be specified with certainty.

Table 1. Group compositions, observation times, and dates of birth of infants for seven studied groups.

\begin{tabular}{|c|c|c|c|c|c|c|}
\hline Group & $\begin{array}{l}\text { Study } \\
\text { period }\end{array}$ & $\begin{array}{l}\text { Group } \\
\text { composition }\end{array}$ & $\begin{array}{l}\text { Infant date } \\
\text { of birth }\end{array}$ & $\begin{array}{l}\text { Total/focal } \\
\text { sampling } \\
\text { time (h) }\end{array}$ & $\begin{array}{l}\text { focal time (h) } \\
\text { with/without } \\
\text { infant } \\
\text { days observed } \\
\text { with/without } \\
\text { infant }\end{array}$ & $\begin{array}{l}\text { focal time (h) } \\
\text { with/without } \\
\text { infant } \\
\text { days observed } \\
\text { with/without } \\
\text { infant }\end{array}$ \\
\hline & & & & & Adult male & Adult female \\
\hline \multirow[t]{2}{*}{1} & $\begin{array}{l}\text { Jun-Dec } \\
2017\end{array}$ & $\begin{array}{l}\text { AM, AF, SM, } \\
\text { Juv }\end{array}$ & - & \multirow[t]{2}{*}{$482.4 / 139.7$} & \multirow[t]{2}{*}{$\begin{array}{l}77.6 / 12.2 \\
52 / 12\end{array}$} & \multirow[t]{2}{*}{$\begin{array}{l}46.6 / 3.3 \\
41 / 11\end{array}$} \\
\hline & $\begin{array}{l}\text { Sep-Dec } \\
2018\end{array}$ & $\begin{array}{l}\text { AM, AF, SM, } \\
\text { Juv, Inf }\end{array}$ & 5 Jun 2018 & & & \\
\hline \multirow[t]{2}{*}{2} & $\begin{array}{l}\text { Sep-Oct } \\
2017\end{array}$ & $\mathrm{AM}, \mathrm{AF}$ & - & \multirow[b]{2}{*}{$479.9 / 49.3$} & \multirow[t]{2}{*}{$\begin{array}{l}16.5 / 11.6 \\
24 / 18\end{array}$} & \multirow[t]{2}{*}{$\begin{array}{l}14.8 / 6.4 \\
26 / 16\end{array}$} \\
\hline & $\begin{array}{l}\text { Jul 2018, } \\
\text { Oct-Dec } \\
2018\end{array}$ & $\mathrm{AM}, \mathrm{AF}, \mathrm{Inf}$ & 26 Oct 2018 & & & \\
\hline \multirow[t]{2}{*}{3} & $\begin{array}{l}\text { Oct-Dec } \\
2017\end{array}$ & $\begin{array}{l}\text { AM, AF, Juv, } \\
\text { Inf }\end{array}$ & Sept 2017 & \multirow[b]{2}{*}{$393.6 / 20.8$} & \multirow[t]{2}{*}{$\begin{array}{l}3.4 / 5.5 \\
7 / 17\end{array}$} & \multirow[t]{2}{*}{$\begin{array}{l}3.1 / 8.8 \\
8 / 22\end{array}$} \\
\hline & Jun 2018 & $\begin{array}{l}\text { AM, AF, SM, } \\
\text { Juv }\end{array}$ & - & & & \\
\hline 4 & $\begin{array}{l}\text { Jun-Jul } \\
2018, \\
\text { Sep-Oct } \\
2018\end{array}$ & AM, AF, Juv & - & $387.2 / 86.0$ & $\begin{array}{l}46.8 /- \\
44 /-\end{array}$ & $\begin{array}{l}39.2 /- \\
41 /-\end{array}$ \\
\hline 5 & $\begin{array}{l}\text { Aug-Oct } \\
2018\end{array}$ & AM, AF, Juv & - & $294.8 / 38.3$ & $\begin{array}{l}23.5 /- \\
27 /-\end{array}$ & $\begin{array}{l}14.8 /- \\
28 /-\end{array}$ \\
\hline \multirow[t]{2}{*}{6} & $\begin{array}{l}\text { Aug-Oct } \\
2017\end{array}$ & $\begin{array}{l}\text { AM, AF, SM, } \\
\text { SF, Juv, Inf }\end{array}$ & 5 Oct 2017 & \multirow[t]{2}{*}{$520.3 / 38.4$} & \multirow[t]{2}{*}{$\begin{array}{l}22.5 / 3.6 \\
27 / 4\end{array}$} & \multirow[t]{2}{*}{$\begin{array}{l}10.8 / 1.5 \\
25 / 2\end{array}$} \\
\hline & Jul 2018 & $\begin{array}{l}\text { AM, AF, SM, } \\
\text { SF, Juv }\end{array}$ & - & & & \\
\hline \multirow[t]{2}{*}{7} & Aug 2018 & $\mathrm{AM}, \mathrm{AF}$ & - & \multirow[t]{2}{*}{$192.6 / 12.8$} & \multirow{2}{*}{$\begin{array}{l}2.0 / 6.5 \\
3 / 10\end{array}$} & \multirow{2}{*}{$\begin{array}{l}0.6 / 3.7 \\
4 / 9\end{array}$} \\
\hline & Nov 2018 & $\mathrm{AM}, \mathrm{AF}, \mathrm{Inf}$ & 1 Nov 2018 & & & \\
\hline
\end{tabular}

AM - adult male, AF - adult female, SM - subadult male, SF - subadult female (distinguishable by size from adults), Juv - juvenile ( $>4,5$ months), Inf - infant ( $<4.5$ months). Sex could not be determined for juveniles and infants due to small genital size. 


\section{Statistical analyses}

To characterize activity patterns, we calculated daily proportions of time spent in each activity category for the adult male and female of each group. To do this, we summed the durations of time spent in each activity category during all focal observations during the day and divided the sum by the total daily observation time. The use of proportions allowed us to account for the effect of different durations of daily observations. To characterize diet composition, we calculated daily proportions of feeding time allocated to fruits, arthropods or other types of food (combining leaves, shoots, flowers and soil) for the adult male and female of each group.

\section{Infant care, activity budgets and diet composition}

To examine the effects of infant care in females and males, we used generalized linear mixed models (GLMM; Baayen, 2008) with beta error structure and logit link function (since our response is a true continuous proportion). We ran five models with the following daily proportions as response variables: (1) feeding time; (2) resting time; (3) social time; (4) moving time; (5) feeding time allocated to arthropods. In each model, we included presence of a dependent infant (hereafter 'infant presence') and sex as fixed-effect predictors and group ID as a random-effect predictor to account for the repeated observations. To control for their possible effects, we included rainfall data as a measure of seasonality (monthly averages in mm at Tamshiyacu $\left(4^{\circ} 00^{\prime} 10.7^{\prime \prime} \mathrm{S}, 73^{\circ} 09^{\prime} 38.2^{\prime \prime} \mathrm{W}\right)$, ca. $40 \mathrm{~km}$ north of EBQB, data available at https://www.worldweatheronline.com) and group size (not including dependent infants) as control predictors. Since we expected the effect of infant presence on the feeding and resting time and diet composition to be the opposite in males and females, we also included interaction between infant presence and sex in the respective models (1, 2 and 5). We compressed all the responses by taking $\mathrm{y}^{\prime}=(\mathrm{y} \times(N-1)+0.5) / N$, where $N$ is the sample size (Smithson \& Verkuilen 2006). This transformation allowed us to avoid zeros and ones (since their logits are undefined) but did not change the interior distribution shape (see more details on this practical issue in Smithson \& Verkuilen (2006). To achieve an approximately symmetrical distribution, we further square root-transformed the response. We ztransformed group size and rainfall (Schielzeth 2010). To reduce the probability of type I error (Barr et al. 2013), we included random slopes of season and sex within group. Originally we also included the correlations of random slopes of season and sex with the intercepts, but they proved to be unidentifiable (Matuschek et al. 2017), so we removed them from the models.

We tested the overall effect of test predictors using full-null model comparison based on a likelihood ratio test (Dobson et al. 2008; Forstmeier \& Schielzeth 2011). The null models for 
models 1, 2 and 5 lacked the predictor of infant presence and its interaction with sex; null models for models 3 and 4 lacked the predictors of infant presence and sex; otherwise the null models were identical to the full models. We tested the individual fixed-effect predictors using likelihood ratio tests comparing the full model with reduced models lacking each of the predictors one at a time (Barr et al. 2013). To check for collinearity between predictors, we determined variance inflation factors (VIF; Quinn \& Keough, 2002) with the function vif of the package car (version 3.0.2; Fox \& Weisberg, 2011). It revealed no collinearity (maximum VIF 1.61 for models 1, 2 and 5; 1.60 for models 3 and 4; Quinn \& Keough, 2002).

\section{Effect of lactation on feeding}

To test whether feeding time changed in lactating females compared to nonlactating females, we ran two GLMMs with beta error structure and logit link function. We used daily proportions of feeding time as response variable and lactation presence as a fixed-effect predictor. For the first model, we considered lactation to be present only until the beginning of weaning (= period of infant dependency: until an infant is 19 weeks old). For the second model, we considered lactation to be present for the whole lactation period, until the infant is 28 weeks old. In each model, we included rainfall and group size as control predictors and group ID as a random-effect predictor. We also included the random slope of rainfall within group. Transformations of response and predictors were identical to that of the models described above.

\section{Trade-offs between activity categories in lactating females}

To test for trade-offs between activity categories in lactating females, we ran a GLMM with beta error structure and logit link function using data for the high-intensity lactation period (until an infant is 28 weeks old). We ran three models with the following daily proportions as response variables: (1) resting time; (2) social time; (3) moving time. In each model, we included daily proportions of time feeding (z-transformed) as a response and group ID as a random-effect predictor. We compressed the responses using the formula specified above and further square root-transformed time spent socializing and time spent moving. Null models used for full-null model comparison were intercept-only models.

All models were fitted in R (version 3.5.3; R Core Team, 2018) using the package glmmTMB (version 0.2.3; Brooks et al., 2017). To assess model stability, we compared the full model estimates with those obtained from models with the levels of the random-effect predictors excluded one at a time using a function provided by R. Mundry (Leipzig, Germany). The results of stability and overdispersion tests, as well as sample sizes, are specified in Table 2 and Appendix Tables A1 and A2. 


\section{Results}

Infant care, activity budgets and diet composition

Both activity budgets and diet composition were different before and after infant birth (Table 2, Fig. 1-3, Appendix Table A1). For feeding, resting time and the proportion of feeding time allocated to arthropods, the direction of changes was opposite for females and males. For social and moving time, the direction of changes was similar for females and males.

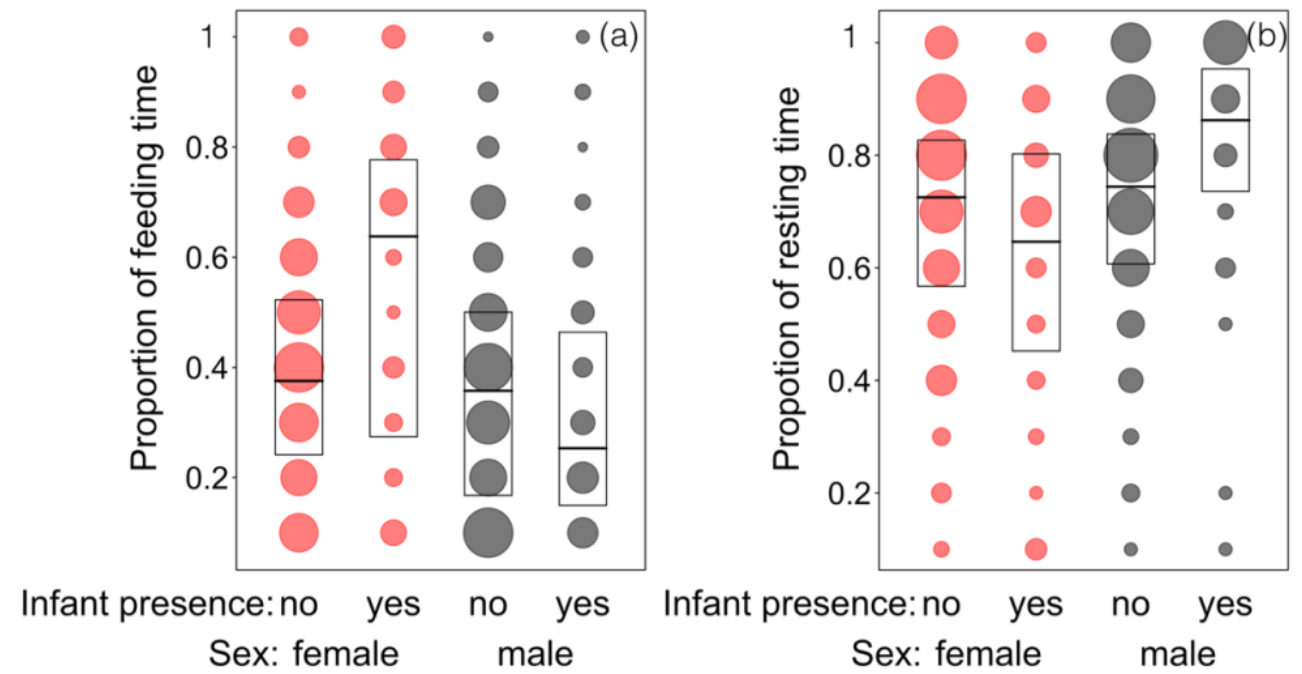

Fig. 1. Daily proportions of (a) feeding time and (b) resting time of all females and males in relation to infant presence/absence. For visual clarity proportions were binned into 10 sections. The size of symbols corresponds to the respective number of observations: (a) 1-38; (b) 2-43 per bin per combination of sex and dependent infant absence/presence). Boxes depict the median and lower and upper quartiles.

After infant birth, females fed more (all five females that gave birth during the study) and rested less (three of the five females) than before. Males, in contrast, fed less (four of five males) and rested more (four of five males; Fig. 1, Appendix Table A1). In the GLMM, infant presence, sex and their interaction had a clear association with feeding time (full-null model comparison: $\chi^{2}{ }_{2}=12.461, P=0.002$ ) and resting time (full-null model comparison: $\chi^{2}{ }_{2}=20.051$, $P<0.001)$. Rainfall and group size did not have an association with feeding or resting time (Table 2).

After infant birth, both sexes spent less time socializing (all females, four of five males) and less time moving (four of five females, all males) than before (Fig. 2, Appendix Table A1). In the GLMM, infant presence and sex had a clear association with social time (full-null model comparison: $\left.\chi^{2}{ }_{2}=24.572, P<0.001\right)$. Infant presence did not have an association with moving time, but sex did: females moved more than males, especially after infant birth (Table 2). 


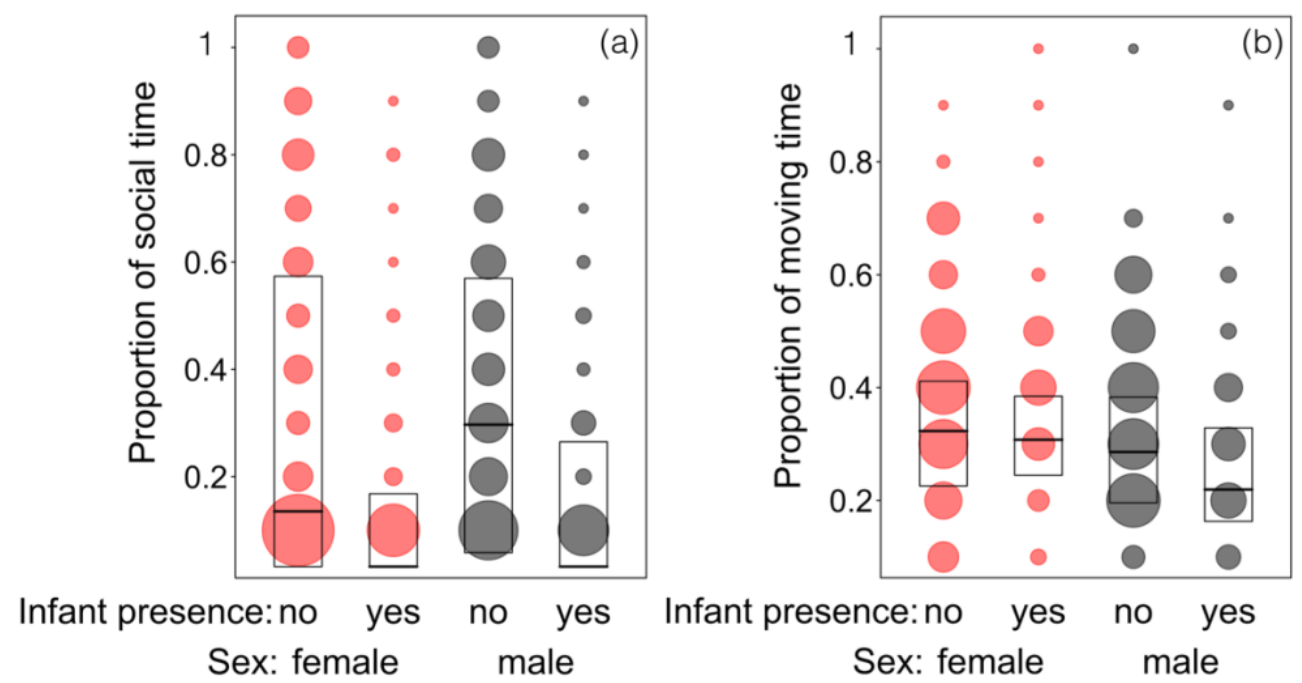

Fig. 2. Daily proportions of (a) social time and (b) moving time of all females and males in relation to infant presence/absence. For visual clarity proportions were binned into 10 sections. The size of symbols corresponds to the respective number of observations: (a) 1-86; (b) 1-45 per bin per combination of sex and infant absence/presence). Boxes depict the median and lower and upper quartiles.

After infant birth, females increased the proportion of feeding time allocated to arthropods (four of five females). Males on average decreased the proportion of feeding time allocated to arthropods (although a pronounced decrease was observed only in two of five males; Fig. 3, Appendix Table A1). In the GLMM, infant presence, sex and their interaction also had a clear association with diet composition (full-null model comparison: $\chi^{2}{ }_{2}=6.428, P=0.04$ ). The effect was opposite in females and males. Females consumed more arthropods after infant birth than before, while males consumed fewer (Fig. 3, Table 2).

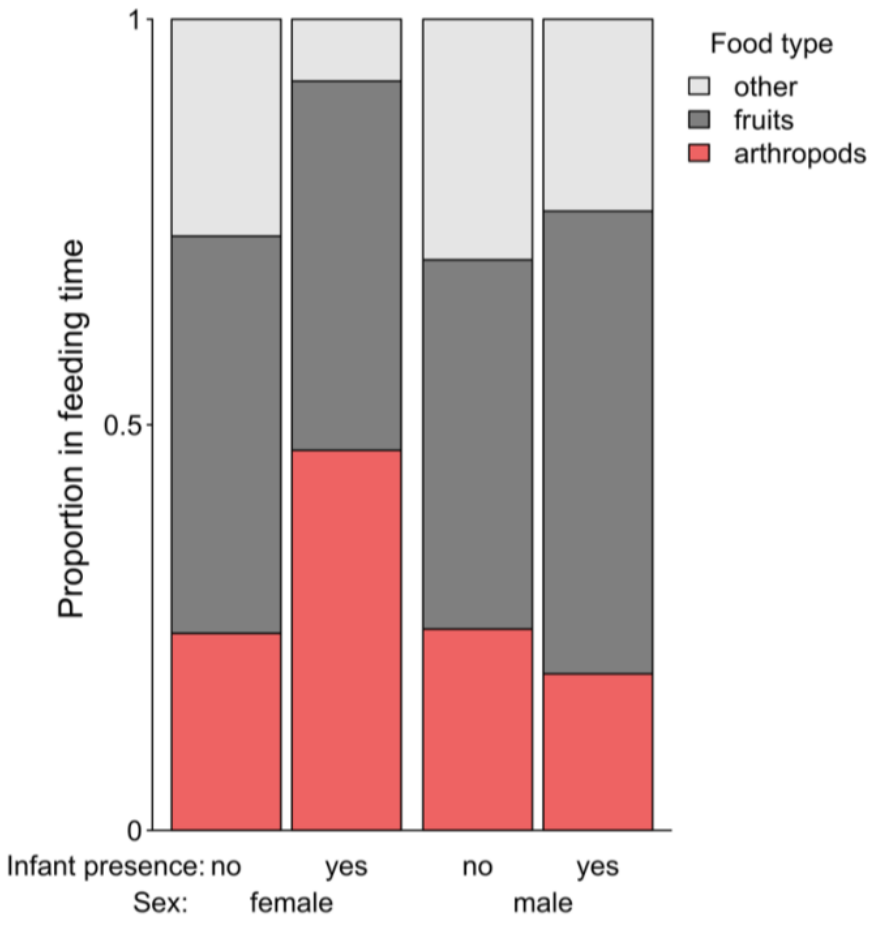

Fig. 3. Mean daily proportions of feeding time allocated for arthropods, fruits and other items (flowers, leaves, shoots, and soil) of all females and males in relation to infant presence/absence. 
Table 2. Results of the models of the daily proportion of (1) feeding time; (2) resting time; (3) social time; (4) moving time; (5) feeding time allocated to arthropods, in females and males. Indicated are estimates and standard errors, confidence intervals, results of likelihood ratio tests, and the range of estimates obtained when dropping levels of random effects one at a time.

\begin{tabular}{|l|l|l|l|l|l|l|l|l|l|l|l|}
\hline Term & Estimate & SE & $\begin{array}{l}\text { Lower } \\
\text { CI }\end{array}$ & $\begin{array}{l}\text { Upper } \\
\text { CI }\end{array}$ & $\chi 2$ & df & P-value & min & max \\
\hline \\
$\begin{array}{l}\text { Model 1: feeding time in females and males } \\
\text { dispersion parameter }=0.91 ;\end{array}$ 799 daily proportion values from 7 groups \\
\hline Intercept & -0.661 & 0.114 & -0.886 & -0.437 & - & - & - & -0.748 & -0.536 \\
\hline Infant presence (1) & -0.197 & 0.194 & -0.576 & 0.183 & - & - & $(2)$ & -0.396 & -0.001 \\
\hline Sex (3) & 0.154 & 0.139 & -0.118 & 0.426 & - & - & $(2)$ & 0.064 & 0.259 \\
\hline Group size (4) & 0.069 & 0.093 & -0.114 & 0.252 & 0.602 & 1 & 0.438 & -0.096 & 0.145 \\
\hline Rainfall (5) & -0.160 & 0.223 & -0.597 & 0.278 & 0.555 & 1 & 0.456 & -0.286 & 0.047 \\
\hline $\begin{array}{l}\text { Infant } \\
\text { presence:sex }\end{array}$ & 0.740 & 0.225 & 0.299 & 1.181 & 11.382 & 1 & 0.001 & 0.584 & 0.944 \\
\hline
\end{tabular}

\section{Model 2: resting time in females and males}

dispersion parameter $=0.96 ; 479$ daily proportion values from 7 groups

\begin{tabular}{|l|l|l|l|l|l|l|l|l|l|}
\hline Intercept & 0.774 & 0.142 & 0.496 & 1.052 & - & - & - & 0.685 & 1.025 \\
\hline Infant presence (1) & 0.656 & 0.198 & 0.268 & 1.044 & - & - & $(2)$ & 0.258 & 1.075 \\
\hline Sex (3) & -0.111 & 0.095 & -0.296 & 0.075 & - & - & $(2)$ & -0.153 & -0.055 \\
\hline Group size (4) & -0.064 & 0.115 & -0.289 & 0.160 & 0.326 & 1 & 0.568 & -0.208 & 1.165 \\
\hline Rainfall (5) & 0.104 & 0.125 & -0.141 & 0.348 & 0.803 & 1 & 0.370 & 0.015 & 0.470 \\
\hline $\begin{array}{l}\text { Infant } \\
\text { presence:sex }\end{array}$ & -0.815 & 0.191 & -1.190 & -0.441 & 17.988 & 1 & 0.000 & -1.132 & -0.516 \\
\hline
\end{tabular}

\section{Model 3: social time in females and males}

dispersion parameter $=1.12 ; 479$ daily proportion values from 7 groups

\begin{tabular}{|l|l|l|l|l|l|l|l|l|l|}
\hline Intercept & -0.616 & 0.121 & -0.852 & -0.379 & - & - & - & -0.774 & -0.468 \\
\hline Infant presence (1) & -0.814 & 0.178 & -1.164 & -0.465 & 20.506 & 1 & 0.000 & -0.957 & -0.530 \\
\hline Sex (3) & -0.200 & 0.099 & -0.394 & -0.005 & 4.053 & 1 & 0.004 & -0.243 & -0.154 \\
\hline Group size (4) & -0.155 & 0.095 & -0.342 & 0.031 & 2.562 & 1 & 0.110 & -0.246 & 0.020 \\
\hline Rainfall (5) & -0.038 & 0.069 & -0.172 & 0.097 & 0.301 & 1 & 0.583 & -0.145 & 0.011 \\
\hline
\end{tabular}

\section{Model 4: moving time in females and males}

dispersion parameter $=0.93 ; 479$ daily proportion values from 7 groups

\begin{tabular}{|l|l|l|l|l|l|l|l|l|l|}
\hline Intercept & -0.922 & 0.069 & -1.058 & -0.786 & - & - & - & -1.017 & -0.868 \\
\hline Infant presence (1) & -0.037 & 0.107 & -0.246 & 0.173 & 0.120 & 1 & 0.730 & -0.173 & 0.083 \\
\hline Sex (3) & 0.145 & 0.058 & 0.032 & 0.259 & 5.576 & 1 & 0.018 & 0.109 & 0.175 \\
\hline Group size (4) & 0.019 & 0.057 & -0.093 & 0.132 & 0.110 & 1 & 0.740 & -0.022 & 0.135 \\
\hline Rainfall (5) & -0.117 & 0.057 & -0.228 & -0.006 & 4.146 & 1 & 0.042 & -0.143 & -0.066 \\
\hline
\end{tabular}


Model 5: feeding time allocated to arthropods in females and males

dispersion parameter $=1.02 ; 356$ daily proportion values from 7 groups

\begin{tabular}{|l|l|l|l|l|l|l|l|l|l|}
\hline Intercept & 0.124 & 0.182 & -0.233 & 0.481 & - & - & - & -0.001 & 0.277 \\
\hline Infant presence (1) & -0.575 & 0.301 & -1.165 & 0.015 & - & - & $(2)$ & -0.791 & -0.403 \\
\hline Sex (3) & 0.116 & 0.200 & -0.275 & 0.508 & - & - & $(2)$ & -0.036 & 0.246 \\
\hline Group size (4) & -0.186 & 0.147 & -0.475 & 0.102 & 1.615 & 1 & 0.204 & -0.365 & -0.071 \\
\hline Rainfall (5) & 0.140 & 0.235 & -0.321 & 0.601 & 0.377 & 1 & 0.539 & -0.011 & 0.270 \\
\hline $\begin{array}{l}\text { Infant } \\
\text { presence:sex }\end{array}$ & 0.807 & 0.330 & 0.160 & 1.454 & 5.852 & 1 & 0.016 & 0.629 & 0.872 \\
\hline
\end{tabular}

(1) Dummy coded with absence of infant being the reference category

(2) Not shown because of having very limited interpretation for a GLMM with interaction

(3) Dummy coded with male being the reference category

(4) Z-transformed to a mean of 0 and a standard deviation of 1; mean and sd of the original value were 3.216 and 0.877 , respectively

(5) Z-transformed to a mean of 0 and a standard deviation of 1; mean and sd of the original value were 91.754 and 79.121, respectively

\section{Effect of lactation on feeding time}

Lactating females spent more time feeding than nonlactating females, especially in the first 19 weeks after birth (full-null model comparison: $\chi^{2}{ }_{1}=6.610, P=0.010$ ). When analysing the entire period of high-intensity lactation (until an infant is 28 weeks old), the association was less pronounced (Appendix Table A2). The effect of lactation on feeding time, therefore, was stronger in the first 4.5 months of lactation. The increase in feeding time was nonlinear: it increased right after infant birth, dropped around week 2 and started to rise again from week 7, reaching its peak around week 17 (Fig. 4).

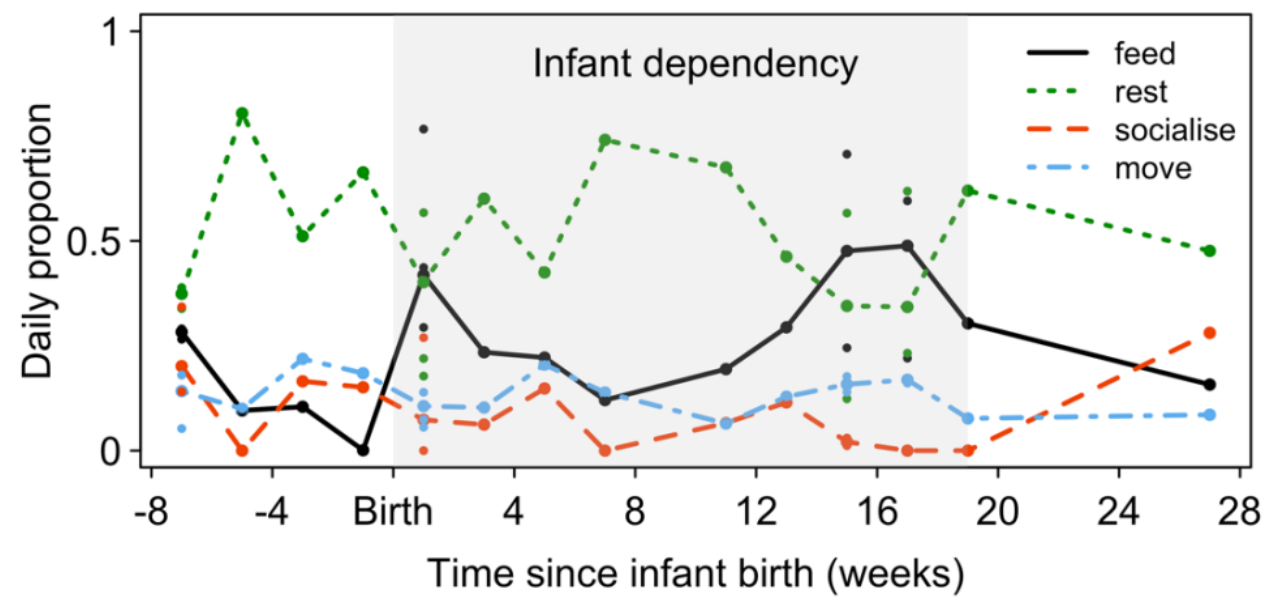

Fig. 4. Daily proportion of time females spent in each of the activities; dots connected by lines represent mean values for all females for a 2-week block; other dots show mean values for each individual female. The plot encompasses the period of high intensity lactation ( 28 weeks), including the period of infant dependency (19 weeks; grey area), and 8 weeks before infant birth for comparison. Because the first week of infant's life happens between the age of 0 and 1 weeks, the data points are located between the ticks indicating infant age. 


\section{Trade-offs between activities in lactating females}

There was a significant negative relationship between feeding time and both resting (full-null model comparison: $\chi^{2}{ }_{1}=47.797, P<0.001$ ) and social (full-null model comparison: $\chi^{2}{ }_{1}=8.192$, $P=0.004$ ) time. The relationship between feeding and moving time (full-null model comparison: $\chi^{2}{ }_{1}=3.578, P=0.06$ ) was not significant (Fig. 5). The relationship was much stronger for resting time (slope $=-0.905$ ) than for social time (slope $=-0.363$ ). Lactating females, therefore, drew extra time for feeding primarily from resting time and, to a lesser extent, from social time (Fig. 4, 5, Appendix Table A3).
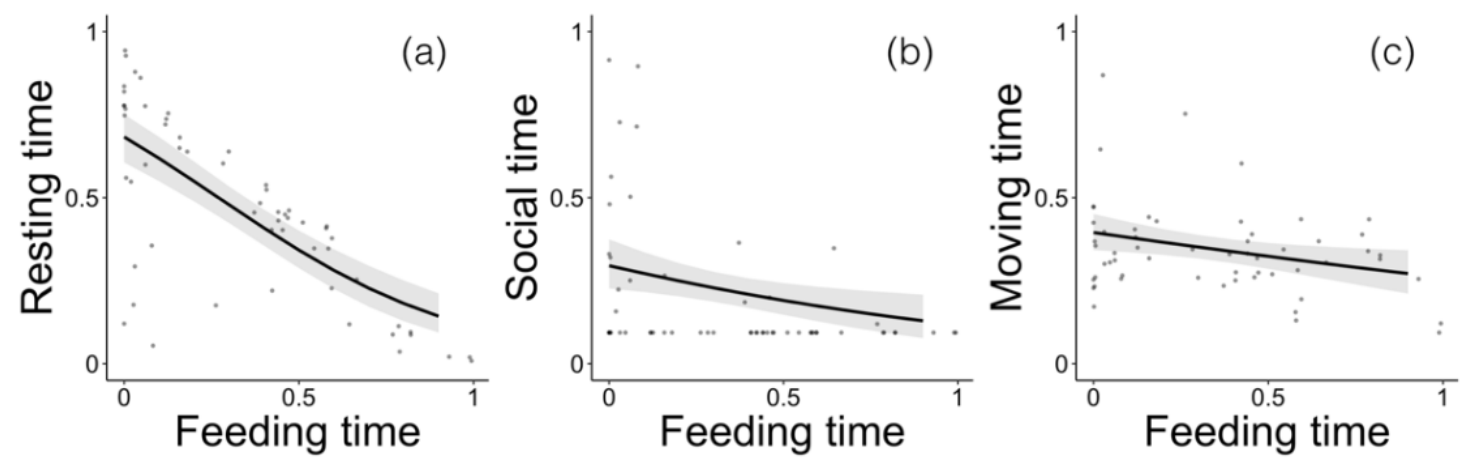

Fig. 5. Daily proportions of (a) resting, (b) social, and (c) moving time, as a function of daily proportion of feeding time, for the period of high intensity lactation ( 28 weeks).

\section{Discussion}

The presence of a dependent infant affected activity patterns and diet composition in both sexes, but the direction of the changes was mostly the opposite in females and males. While females fed more, consumed more arthropods and rested less after infant birth than before, males fed less, consumed fewer arthropods and rested more after infant birth. Only social time was affected in the same way in both sexes: both females and males socialized less after infant birth than before.

These changes in activity patterns represent two different strategies of dealing with the energetic costs, the energy-maximizing 'eat more' strategy for the females and the energyminimizing 'do less' strategy for males. Similar contrasting strategies have been observed in tamarins where males carry the infants: while lactating females fed more, infant-carrying males fed less and rested more (saddleback, Goldizen, 1987; cotton-top, Price, 1992b).

Why would males in these species not use the more straightforward 'eat more' strategy to offset the energetic costs of infant carrying and instead risk a reduction in body condition? First, infant carrying seems to be in conflict with efficient foraging, particularly arthropod foraging (Tardif 1994). Both in our study and in Wright's (1984) study of dusky titi monkeys, males consumed fewer arthropods during the infant dependency period. In contrast, lactating 
females, not handicapped by infants, could afford to forage for more arthropods during this period. Second, efficient foraging might be incompatible with vigilance. A trade-off between foraging and vigilance is well known (Miller 2002; Lima 1987), and increased vigilance in the presence of infants has been shown for many mammals. In chacma baboons, females increased their vigilance while lactating and carrying, and there was a negative relationship between feeding time and vigilance (Barrett et al. 2006). In cooperatively breeding meerkats, Suricata suricatta, helpers that guarded infants had increased vigilance levels, spent less time foraging and found less food (Clutton-Brock et al. 1998; Santema \& Clutton-Brock 2013). Moreover, in many primate species males have been shown to be generally more vigilant than females (Isbell 1994; van Schaik \& van Noordwijk 1989). Vigilance has not been quantified in titis, but both in our study and in Lawrence's (2007) study on dusky titi monkeys, males appeared to be more vigilant than females, often scanning the environment while the rest of the group was feeding, and male white-tailed titis, Plecturocebus discolor, showed antipredator behaviour only in the presence of infants (De Luna et al. 2010). Infant-carrying males must be more vulnerable to predation not only because infants are more likely to be targeted by predators, but also because a male, handicapped by an infant, will be slower in escaping the predator himself.

It has been suggested that foraging would be more affected in species using crypsis as a main antipredator strategy (Tardif 1994). As small (adult body mass around $1 \mathrm{~kg}$ ), cryptically coloured primates living in small groups, titis use hiding and fleeing as their main antipredator strategies (Wright 1984). Therefore, foraging must be especially strongly affected in infant-carrying male titis. Indeed, in squirrel monkeys, which live in large groups and thus can afford to have reduced individual vigilance levels due to the group size effect (van Schaik 1983), there was no effect of maternal carrying on foraging rates (Boinski 1988). Unlike males, lactating females are not handicapped by infants or by the need for increased vigilance and thus can afford to increase feeding time and consume more protein-rich arthropods. These changes in female behaviour have been found for red (Tirado Herrera \& Heymann 2004) and dusky (Wright 1984) titi monkeys, vervet monkeys, Chlorocebus aethiops (Lee 1987), and mantled howlers, Alouatta palliata (Serio-Silva et al. 1999), consistent with the fact that protein requirements are strongly increased during lactation (Buttery 1979).

While lactating females generally increased their feeding time compared to nonlactating females, this increase was not linear and was most pronounced in the first 4.5 months of lactation, until the beginning of weaning. Right after infant birth, feeding time increased, but then dropped again around week 2 and started to increase again from ca. week 12 (2.8 
months), reaching its peak almost at the end of the infant dependency period, around week 17 (4 months).

The feeding time is expected to vary throughout lactation as a function of infant's energy requirements, other costs of maternal care (e.g. infant carrying), female cycling (e.g. a new pregnancy) and food availability. The effect of a new pregnancy, however, can be excluded in titis since they have lactational amenorrhea and do not become receptive again until around 7.5 months after giving birth (about a month after weaning; Valeggia et al., 1999). The effect of food availability can be excluded as well, as infant births were not seasonal in our study, with birthdates ranging from June (dry season) to November (wet season), and seasonality did not have any effect on maternal feeding time in the models. The variation in feeding time should then be explained by a combination of infant growth and other costs of care.

During the first weeks of an infant's life, lactation is most intensive, with nursing occurring every $2 \mathrm{~h}$ on average (Wright 1990). In addition, in the first week mothers may contribute to infant carrying to some degree (about $20 \%$ of carrying time in a captive population: Jantschke et al., 1995; about 5\% on average during first 3 weeks in a wild population: Wright, 1984). The increase in female feeding time in the first 2 weeks following an infant's birth can probably be attributed to the combined energetic demands of lactation and infant transport.

The second increase in feeding time at weeks 13-21 is harder to explain. It roughly coincides with the period of pronounced changes in the infant's behaviour: between 13 and 21 weeks, an infant gradually becomes more independent, starting to play with siblings and travel on its own at ca. 15 weeks, travelling independently at the age of ca. 19 weeks and switching to adult forms of contact with parents (tail twining, grooming and sitting next to, rather than on, a parent) at the age of 17-21 weeks (Jantschke et al. 1995; Fragaszy et al. 1982; Wright 1984). During this period (13-21 weeks), lactating females most often resist infants' attempts to nurse (Fragaszy et al. 1982). Given that maternal investment can be measured in terms of conflict between mothers and infants (Lee 1987), the high frequency of suckling rejections indicates that during this period the energetic costs of lactation become too high for a female. However, the costs of lactation are the highest at peak lactation (Altmann 1980), which occurs before an infant starts to consume supplemental foods (Lee et al.1991). In titis, peak lactation happens about 1-1.5 months after birth (Wright 1984; Fragaszy et al. 1982), so it cannot account for the increase in feeding time at weeks 13-21. Additional data on suckling frequency and diet composition of mothers and infants might help address this issue.

Contrary to the predictions of Altmann's model, lactating females in our study drew extra time for feeding primarily from resting time and, to a lesser extent, from social time. The same effect has been shown in baboons, geladas and black howlers (Dunbar \& Sharman 1984; 
Dunbar \& Dunbar 1988; Dias et al. 2011), where extra feeding time was drawn mainly from resting time and, only when it was exhausted, from social time. In all these species, the risk of infanticide by males is high and females are subject to harassment from other group members, so surrendering social time might have serious consequences for females (Dunbar \& Dunbar 1988). However, conserving social time was also observed in red titis in our study and in cotton-top tamarins (Price 1992a), even though no infanticide has been recorded for either of these species and females are not at risk of harassment from other group members. In both species, however, females receive a lot of help from other group members: males in titis and all adult and subadult group members in tamarins. Presumably, the value of these services encourages the females to contribute to maintaining relationships with the helpers and to conserve social time even in the absence of risks of infanticide or harassment.

Note that social time is not homogeneous and consists of components with different weights (Dunbar \& Dunbar 1988). Even though social time dropped for both sexes in our study, females conserved the amount of grooming directed at their pair mates (Dolotovskaya, Walker, et al. 2019). As a result, grooming between pair mates, while being almost reciprocal before infant birth, was heavily skewed towards female investment during infant dependency, suggesting the importance of pair bond maintenance to females. Similarly, in chacma baboons lactating females conserved the diversity of social partners even when they had to decrease social time (Barrett et al. 2006), geladas conserved social time devoted to primary partners, but drew from time devoted to casual acquaintances when necessary (Dunbar \& Dunbar 1988), and cotton-top tamarins, while decreasing active investment in grooming, conserved the total social time (Price, 1992a). In yellow baboons, females decreased their investment in grooming during lactation, but strongly increased it immediately after weaning (Altmann 1980). All these examples suggest that, even when lactating females are forced to give up social time, they try to conserve 'quality social time', expressed as either active investment in grooming or time devoted to the most important social partners, or, when that is not possible, to repay the debt later.

In conclusion, our study suggests that in red titi monkeys, females and males use opposing strategies to deal with the costs of parental care. While lactating females use the 'eat more' strategy, feeding more, catching more arthropods and resting less after infant birth than before, males use the 'do less' strategy, feeding less, catching fewer arthropods and resting more after infant birth. Lactating females drew the extra feeding time mainly from resting time and, to a lesser degree, from social time. Despite the general decrease in social time, females conserved grooming time directed at their pair mates, indicating the importance of pair-bond maintenance to females. 


\section{CHAPTER 6: ACTIVE ANTI-PREDATOR BEHAVIOUR IN RED TITI MONKEYS}

(Plecturocebus cupreus)

Sofya Dolotovskaya ${ }^{1,2}$, Eckhard W. Heymann ${ }^{1}$

Sofya Dolotovskaya1, 2, Camilo Flores Amasifuen, ${ }^{3}$ Caroline Elisabeth Haas ${ }^{4}$, Fabian Nummert ${ }^{4}$, Eckhard W. Heymann ${ }^{1}$

\footnotetext{
${ }^{1}$ Behavioural Ecology and Sociobiology Unit, German Primate Center, Göttingen, Germany

2 Primate Genetics Laboratory, German Primate Center, Göttingen, Germany

${ }^{3}$ Estación Biológica Quebrada Blanco, Quebrada Blanco, Río Tahuayo, Peru

${ }^{4}$ Göttingen University, Göttingen, Germany
}

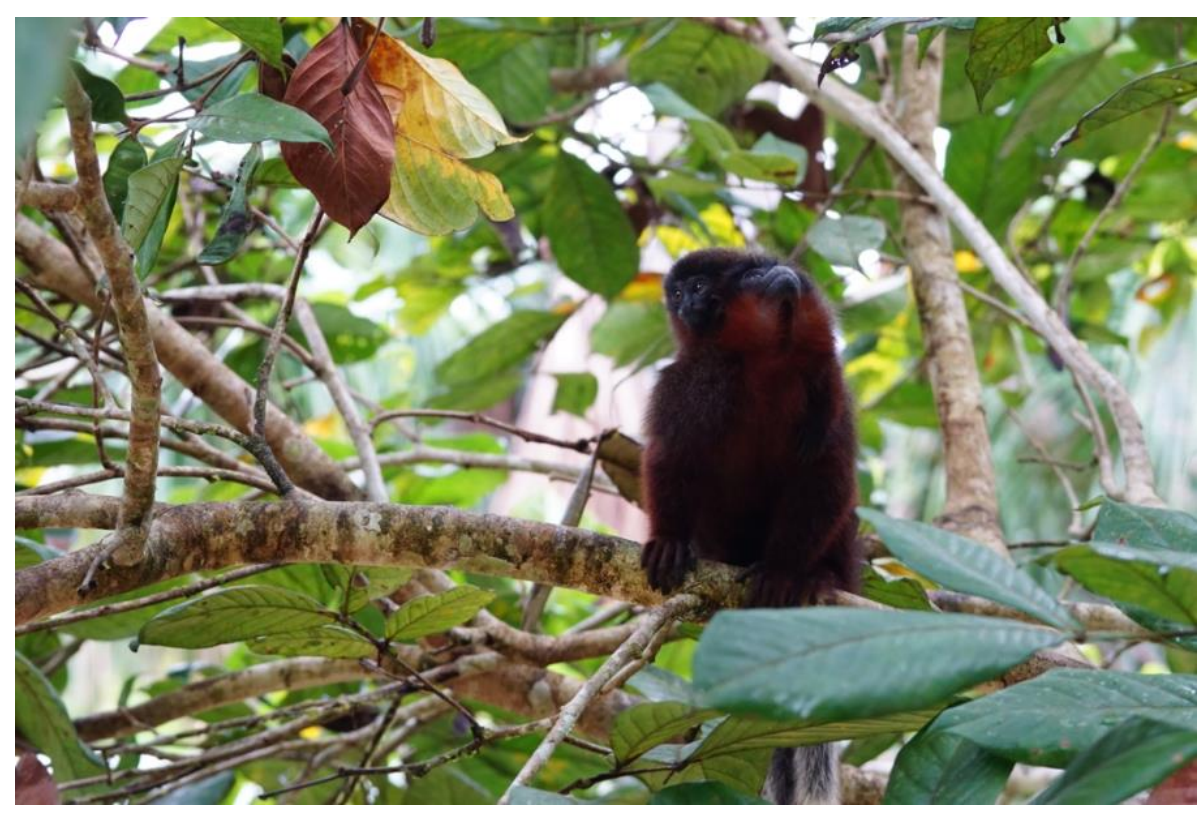

\section{Primate Biology}

https://doi.org/10.5194/pb-6-59-2019

(Accepted 25 February 2020) 


\begin{abstract}
Due to their inconspicuous behaviour and coloration, it has been assumed that titi monkeys' main anti-predator behaviour is passive crypsis and hiding. So far, active predator mobbing has been documented only for black-fronted titi monkeys, Callicebus nigrifrons. Here we report for the first time mobbing behaviour of red titi monkeys, Plecturocebus cupreus (previously Callicebus cupreus), as reaction to an ocelot (Leopardus pardalis) and a Boa constrictor. We also report other active antipredator behaviours, such as alarm calling and approaching, as reactions to tayras (Eira barbara) and raptors. Our observations provide additional evidence for sex differences in antipredator behaviour, possibly related to the evolution and maintenance of social monogamy.
\end{abstract}

\title{
1. Introduction
}

Although predation is thought to play a major role in the evolution of primate behaviour and ecology, predation on primates is rarely directly observed in the wild. Encounters with potential predators, however, are observed more often, and reactions of primates to the presence of potential predators might help to estimate the extent of predation pressure by different types of predators.

Behavioural responses to predators vary considerably in primates depending on both predator and prey species. Types of responses can be broadly classified into two groups: passive (e.g., avoidance, fleeing, or hiding) and active (e.g., alarm calling, attacking, or mobbing) and they vary systematically within species depending on the type of predator (Ferrari 2009). Active responses such as alarm calling and mobbing (the latter defined as repeated and aggressive advances on a predator accompanied by calling and displaying in a conspicuous manner: Dutour et al., 2016) often involve several or all group members and have been documented in various primate species (for example, gelada baboons: Iwamoto et al. 1996, moustached tamarins: Shahuano Tello et al. 2002, chimpanzees: Boesch \& BoeschAchermann 2000). In many species, both pair-living and living in multi-male-multi-female groups, males are more involved in these active anti-predator behaviours than females (Isbell 1994). This special male role against predators has been suggested to account both for the evolution of multi-male primate groups and for the evolution and maintenance of pair-living and pair-bonding in pair-living species (e.g., Crook and Gartlan, 1966; De Luna et al., 2010; van Schaik and Dunbar, 1990).

Titi monkeys are small (body mass around $1 \mathrm{~kg}$ ), cryptically coloured, diurnal Neotropical primates living in small family groups containing an adult pair and 1-3 offspring (BiccaMarques \& Heymann 2013). The infants are carried almost exclusively by adult males (Wright 
1984). Due to both their small body size and low group size, titis are faced with a wide range of predators (Table 1 ).

Table 1. Predation and predation attempts on titi monkeys.

\begin{tabular}{|c|c|c|c|}
\hline Prey species & Predator & Type of observation & Source \\
\hline \multirow[t]{3}{*}{$\begin{array}{l}\text { Plecturocebus } \\
\text { discolor } \\
\text { (Callicebus discolor) }\end{array}$} & Eira barbara (tayra) & Unsuccessful attacks & $\begin{array}{l}\text { De Luna, Sanmiguel, Di } \\
\text { Fiore, \& Fernandez- } \\
\text { Duque, } 2010\end{array}$ \\
\hline & Boa constrictor & Successful predation & $\begin{array}{l}\text { Cisneros-Heredia, León- } \\
\text { Reyes, \& Seger, } 2008\end{array}$ \\
\hline & Harpia harpyja (harpy eagle) & Successful predation & De Luna et al., 2010 \\
\hline $\begin{array}{l}\text { Plecturocebus } \\
\text { moloch } \\
\text { (Callicebus moloch) }\end{array}$ & $\begin{array}{lr}\text { Sapajus } & \text { apella } \\
\text { (Cebus apella) } & \text { (capuchin } \\
\text { monkey) } & \end{array}$ & Successful predation & Sampaio \& Ferrari, 2005 \\
\hline \multirow{6}{*}{$\begin{array}{l}\text { Plecturocebus } \\
\text { toppini } \\
\text { (Callicebus moloch) }\end{array}$} & Boa constrictor & Successful predation & Lawrence, 2007 \\
\hline & $\begin{array}{l}\text { Morphnus guianensis (crested } \\
\text { eagle) }\end{array}$ & Successful predation & $\begin{array}{l}\text { Terborgh 1983; Wright } \\
1985\end{array}$ \\
\hline & $\begin{array}{l}\text { Spizaëtus tyrannus and } \\
\text { Spizaëtus ornatus (ornate } \\
\text { hawk-eagles) }\end{array}$ & Successful predation & $\begin{array}{l}\text { Terborgh 1983; Wright } \\
1985\end{array}$ \\
\hline & $\begin{array}{l}\text { Sapajus macrocephalus } \\
\text { (Cebus apella) }\end{array}$ & Successful predation & Lawrence, 2007 \\
\hline & Leopardus pardalis (ocelot) & Unsuccessful attack & Wright 1985 \\
\hline & $\begin{array}{l}\text { Accipiter bicolor (bicolored } \\
\text { hawk) }\end{array}$ & Unsuccessful attack & Wright 1985 \\
\hline $\begin{array}{l}\text { Plecturocebus } \quad s p . \\
\text { (Callicebus moloch) }\end{array}$ & Harpia harpyja (?) & Successful predation & $\begin{array}{l}\text { Curtis Freese pers. comm. } \\
\text { to Kinzey et al. } 1977\end{array}$ \\
\hline
\end{tabular}

Because of their generally inconspicuous behaviour and coloration, it has been long assumed that the titi monkeys' main anti-predator behaviour was passive crypsis and hiding (Ferrari 2009). More recently, however, active anti-predator group behaviours such as alarm calling and approaching have been documented in titi monkeys: Plecturocebus discolor (CisnerosHeredia et al. 2005; De Luna et al. 2010); Plecturocebus moloch (Sampaio \& Ferrari 2005). Mobbing behaviour in titis has been so far documented only for the black-fronted titi monkey (Callicebus nigrifrons) as reaction to tayras and an unidentified spotted cat (Cäsar et al. 2012). During a field study on the ecology and mating system of red titi monkeys, Plecturocebus cupreus (previously Callicebus cupreus), we witnessed several encounters with different predators/potential predators and recorded the responses of the titis. Here we report for the first time mobbing behaviour of titis as reaction to an ocelot (Leopardus pardalis) and a Boa constrictor. We also report other active antipredator behaviours, such as alarm calling and approaching, and discuss the more active male involvement in these behaviours. 


\section{Materials and Methods}

The study took place at the Estación Biológica Quebrada Blanco (EBQB), in north-eastern Peruvian Amazonia ( $4^{\circ} 21^{\prime} \mathrm{S} 73^{\circ} 09^{\prime} \mathrm{W}$ ). It is embedded in primary tropical rainforest of the tierra firme-type, and includes a small area with anthropogenic secondary forest regenerating since 2001. As part of a study on the mating system and ecology, we habituated and studied seven groups of titi monkeys (Group 1 was habituated and studied intermittently since 1997) between June-December 2017 and June-December 2018. We followed the monkeys from the early morning when the animals left a sleeping site (or from when we could locate the group) until the late afternoon when the animals retired to a sleeping site. Each group was followed by a team of two observers. We documented encounters with predators opportunistically, recording the time, predator taxon when possible, and monkey behaviour during and after the encounters. The total observation time was approximately 2750 hours (from $387 \mathrm{~h}$ to 520 $\mathrm{h}$ for each of the groups mentioned in this study). During the encounters observed in 2017, Group 1 comprised adult male and female, 1 subadult male and 1 juvenile male; in 2018, it comprised adult male and female, 1 subadult male and an infant carried by the adult male. Group 2 comprised adult male and female during all the encounters mentioned here. Group 4 comprised adult male and female and a juvenile during all the encounters mentioned here. Group 6 comprised adult male and female, subadult male and a juvenile during the encounter mentioned here.

\subsection{Statement of Ethics}

All research protocols reported in this manuscript were reviewed and approved by the German Primate Center and the Servicio Nacional Forestal y de Fauna Silvestre (SERFOR) of the Peruvian Ministry of Agriculture (MINAGRI) (permit no.249-2017- SERFOR/DGGSPFFS). All research reported in this manuscript adhered to the legal requirements of the country in which the work took place.

\section{Results}

In total, we observed 12 encounters with potential predators. We also observed 9 encounters with squirrel monkeys (Saimiri cassiquiarensis); since titis showed antipredator response during these encounters, we include them in our report (see discussion below).

\section{Ocelot encounter}

On 17 June 2017 at 6:50 h, Group 1 was moving through the canopy at a height of approximately $10 \mathrm{~m}$. While passing through a big tree, the adult male started alarm calling and was joined after approximately 2-3 minutes by all other group members. We then noticed an ocelot (Leopardus pardalis) lying on a big branch of the same tree. The titis started mobbing 
the ocelot: all the group members surrounded it and began to move erratically around it, emitting alarm calls, lashing tails, swaying heads, and showing piloerection. The titis were moving rapidly towards and away from the ocelot, but never approached closer than 2-3 m. The ocelot did not make any moves towards the titis and kept lying on the branch, but had noticed the human observers and was staring at us from above. The titis kept mobbing the ocelot and vocalizing until 7:38 $\mathrm{h}$, when they left the tree. The ocelot stayed on the same branch without moving. The titis then travelled approximately 50 meters, still emitting alarm calls every 3-5 minutes. At 7:55 h, however, all the group members returned to the same tree and started mobbing the ocelot again, vocalizing, tail-lashing, and moving rapidly towards and away from it. At 8:03 h, the group finally left the tree and proceeded to a feeding tree. The ocelot was still lying on the same branch when the titis left.

\section{Boa encounter}

On 14 July 2017, at 10:00 h, Group 1 was moving through the canopy at a height of approximately $20 \mathrm{~m}$. At a height of approximately $25 \mathrm{~m}$, a boa (Boa constrictor) was lying curled-up on a branch. All group members started to produce alarm calls and to mob the boa, jumping through the canopy around it, making erratic movements towards and away from the snake, tail-lashing and showing piloerection, but never approaching the snake closer than 2-3 m. The mobbing lasted until 10:10 h, when the titis left the tree and moved on through the canopy at a height of approximately $20 \mathrm{~m}$.

\section{Tayra encounters}

On 11 October 2017 at 6:10 h Group 2 entered a fruiting tree and started feeding on fruits. At 6:13 h, the male started alarm calling; a tayra (Eira barbara) was lying in a nearby tree approximately $7 \mathrm{~m}$ from the titis. The male continued vocalizing until 6:34 h, staring at the tayra and from time to time moving towards it, but never approaching it closer than $5 \mathrm{~m}$. The female continued feeding after the male had started alarm calling and only occasionally joined the male in vocalizing, emitting alarm calls for several seconds. At 6:34 h, the tayra left the area. The titis then moved to a nearby tree and rested out of view in a vine tangle until 7:30 h. On the same day, at 14:30 h Group 2 started climbing up a tree approximately $50 \mathrm{~m}$ from the site of the previous encounter with the tayra. At the same time, we saw a tayra moving down the same tree. When the tits noticed the tayra, they turned downwards and moved away quickly while emitting alarm calls. After traveling approximately $30 \mathrm{~m}$, they ran into a group of squirrel monkeys. The titis turned again and fled quickly; we could not find the group again until 15:40 h. 


\section{Birds of prey encounters}

On 19 August 2017, at 10:31 h Group 6 was scattered around foraging at a height of 5-10 m. A juvenile grey-headed kite (Leptodon cayanensis) landed on a tree nearby. The adult male gave alarm calls for about 30 seconds, tail-lashing and displaying piloerection. Until 10:35, when the kite left, the male stayed vigilant and gave one more short alarm call.

On four other occasions in July and August 2018, the adult male and female of Group 4 gave alarm calls simultaneously after encounters with unidentified birds of prey, vocalizing for 1 to $8 \mathrm{~min}$.

On 19 September 2018, Group 1 was resting in a tree at a height of approximately $10 \mathrm{~m}$; the female was separated by more than $10 \mathrm{~m}$ from the other group members. At 7:56 h, an unidentified hawk flew in and perched on another tree approximately $10 \mathrm{~m}$ from the titis. The adult male, who was carrying an infant, started alarm calling. At 8:06 h, the hawk attacked the subadult male. The adult male called again for 2 min and moved downwards into a denser part of the tree crown. At 8:18 h, the hawk attacked the subadult again, and all group members including the female, who had joined the group by then, emitted alarm calls. Shortly after, the hawk left the area. Then the titis rested for about 20 min.

\section{Capuchin and squirrel monkey encounters}

On two occasions in July 2017, Group 1 fled quickly and hid in the vine tangle after hearing large-headed capuchin (Sapajus microcephalus) calls from the distance. On nine occasions in 2017 and 2018, Group 2 fled and hid from squirrel monkey (Saimiri cassiquiarensis) groups; 4 times, Group 2 could not be found for up to 2 days after that the encounter with squirrel monkeys. However, we never observed the titis emitting alarm calls on encounters with capuchins or squirrel monkeys.

\section{Discussion}

\section{Active antipredator behaviour of red titi monkeys}

Here, we observed mobbing behaviour in P. cupreus as a reaction to an ocelot and Boa constrictor. Until now, mobbing has not been described for P. cupreus, but was reported for another titi monkey species, C. nigrifrons, as a reaction to tayras and an unidentified spotted cat (Cäsar et al. 2012). We also observed other active predator responses such as alarm calling and approaching predators; these findings are in agreement with reports on the same behaviours in C. discolor (De Luna et al. 2010; Cisneros-Heredia et al. 2005) and C. moloch (Sampaio \& Ferrari 2005).

The mobbing of ocelot observed here lasted for almost 40 minutes; soon after leaving the area, the titis returned and continued to mob the predator again for almost 10 minutes. 
Interestingly, during both the ocelot and the Boa constrictor encounters, the predators did not make any attempts to attack the monkeys or even move in their direction; both the ocelot and the snake remained exactly at the same spot throughout the encounter, and the ocelot was watching human observers much more intently than the monkeys. Moreover, none of the trees where the predators were encountered had been used as feeding or sleeping trees by the titi monkey group.

One of the adaptive bases for predator mobbing has been hypothesized to be reducing the probability that a predator will attack or remain in the area (Curio 1978). As both ocelots and snakes hunt by ambush and rely on crypsis and surprise for successful predation, mobbing could decrease the probability of a successful ambush by removing the advantage of a surprise attack, either discouraging the predators to attack or inducing them to leave the area. This might be the reason for the titis to actively mob the predators in the trees they were merely passing by, and, in the case of the ocelot, to even come back for a second round of mobbing. This might also be the reason to mob tayras; although never observed in our study, the mobbing of tayras has been reported for C. nigrifrons (Cäsar et al. 2012) and other Neotropical primates (Cäsar \& Zuberbühler 2012). Although tayras, unlike ocelots or snakes, never ambush their prey, they can stalk it (Presley 2000), and thus mobbing could have the same effect on them as on ocelots or snakes. It is noteworthy that during the encounters described here, neither the ocelot nor the snake left the area as a result of mobbing; but neither did they try to attack the titis.

It is assumed that small-bodied primates with cryptic pelage colouration would mainly rely on passive anti-predator strategies such as hiding or fleeing. However, there are multiple reports on mobbing behavior of miniature callitrichids in response to tayras (common marmoset, Callithrix jacchus (Bezerra et al., 2009), buffy-headed marmosets (Ferrari \& Ferrari 1990)) and snakes (pygmy marmosets, Cebuella pygmaea (Soini, 1988), moustached tamarins, Saguinus mystax (Shahuano Tello et al. 2002)). In slightly larger titis and owl monkeys, active anti-predator responses seem to be quite rare, with only few reports available (owl monkeys, Aotus azarae: Savagian and Fernandez-Duque, 2017; C. nigrifrons: Cäsar et al., 2012). This might be explained by the differences in group size, with the range of 4-20 in callitrichids and only 2-7 in titi and owl monkeys. The small groups size and relatively large proportion of immature individuals in titi and owl monkeys probably mainly reinforce cryptic, rather than active anti-predator responses (Ferrari 2009).

Interestingly, although titis are known to produce referential alarm calls, with acoustically distinct variants given to terrestrial or aerial threats (Cäsar \& Zuberbühler 2012), we could not differentiate between different types of calls by listening. 


\section{Active male involvement in the antipredator behaviour}

During 4 out of 10 encounters described here, the adult males were involved in active antipredator behaviours more than other group members: the alarm calling was initiated by adult males and joined by other group members only later. During the tayra encounter, the male was calling and approaching the tayra for more than 20 minutes, while the female continued feeding and only shortly joined the male in alarm calling several times. The females, in contrast, did not initiate the alarm calling or other anti-predator responses on any of the observed encounters. Similar findings were reported by (De Luna et al. 2010), who observed adult males initiating alarm calling on encounters with tayras.

In contrast to De Luna et al., 2010, where active antipredator behaviours were only observed in the presence of infants, only one of the encounters described here happened in the presence of an infant (unidentified hawk, 19 September2018). On this occasion, the male carrying the infant, although emitting alarm calls, did not approach the predator, but hid in the dense part of the tree. All other encounters, including two involving mobbing a predator, happened in the absence of infants.

One of the hypotheses for the evolution and maintenance of pair-living and pair-bonding suggests that it developed as a result of selection for male services, such as direct infant care, protection against predators, or infanticide prevention (e.g., van Schaik and Dunbar, 1990). So far, it is unclear if any significant risk of male infanticide exists in titi monkeys. However, extensive male care combined with more active male involvement in defense in titi monkeys suggests that male services such as direct infant care and protection against predators might have played a role in evolution or maintenance of pair-living and pair-bonding in this species. Reactions to capuchin and squirrel monkeys

On multiple occasions, we observed the titis fleeing and hiding from squirrel monkey $(S$. cassiquiarensis) groups. It might appear an odd behaviour, since squirrel monkeys are mainly frugivorous and insectivorous and have never been reported hunting on primates (Defler 2004). However, squirrel monkeys often form mixed species troops with capuchin monkeys (Podolsky 1990). Capuchin monkeys are known to capture mammals (Fedigan 1990; Fragaszy et al. 2004) and have been directly observed hunting titi monkeys (Lawrence 2007; Sampaio \& Ferrari 2005). Fear reaction to squirrel monkeys is probably explained by titi monkeys associating the presence of squirrel monkeys with the presence of capuchin monkeys. A similar avoidance of and fleeing from squirrel monkeys has been observed in moustached (Saguinus mystax) and black-fronted tamarins (Leontocebus nigrifrons) at EBQB (EWH, personal observations). 
Interestingly, we never observed the titis emitting alarm calls on encounters with capuchin or squirrel monkeys. Since capuchin monkeys, unlike cats or snakes, do not hunt by ambush, passive response such as fleeing and hiding might be more advantageous on encounters with this predator.

\section{Conclusions}

Active antipredator responses described here indicate that, despite their generally cryptic behaviour, titi monkeys' responses to predators are not always passive, as had been long assumed. During 4 out of 10 encounters, adult males showed more active antipredator responses than females or other group members. Our observations put the previous suggestion by (De Luna et al. 2010) on a broader base, namely that protection against predators might have played a role in evolution and maintenance of pair-living and pairbonding in this species. 


\section{CHAPTER 7: GENERAL DISCUSSION}

The preceding chapters combined genetic and behavioral data to examine if social monogamy in coppery titis is translated into genetic monogamy and which proximate mechanisms maintain this social system. In this chapter, I will first summarize the major findings of this thesis. Second, I will bring the different aspects of titis' social system together and discuss the relationships between them. Finally, I will review potential directions for future research.

\subsection{Summary of major findings}

\section{Genetic monogamy, mate choice and dispersal patterns}

Although titi monkeys are often considered a textbook example of a "monogamous" primate, their mating system had not been studied in any wild population until now. My analyses of paternity in 18 offspring of nine family groups found no cases of extra-pair paternities (EPP), although one paternity remained unassigned. This indicates that titis in our study population are mostly genetically monogamous or have very low rate of EPP. To investigate the mechanisms of the genetic monogamy maintenance, I examined if mate choice was based on relatedness or heterozygosity. Relatedness between mating partners in ten observed pairs did not differ from the average relatedness in randomly generated pairs, indicating the absence of active inbreeding avoidance via mate choice. I did not find evidence for heterozygosity-based mate choice either, as the heterozygosity of mating partners was not correlated. Despite the absence of evidence for active inbreeding avoidance via mate choice, pair mates were on average not related (mean $r=-0.033$ ) and never shared the same mtDNA haplotype. To see if low relatedness between mates could be explained by natal dispersal, I analyzed spatial genetic structure of the study population. I showed that dispersal was most likely opportunistic, with both sexes migrating over varying distances. These results indicated that even opportunistic dispersal, as long as it is unconstrained, can be sufficient to prevent inbreeding and thus render active inbreeding avoidance via mate choice unnecessary.

\section{Pair bond and the mechanisms of its maintenance}

Social monogamy and its behavioral correlates are increasingly recognized as consequences of a trade-off between male and female reproductive strategies. To examine the role of males and females in the maintenance of pair living and pair bonds, I analyzed the contributions of both pair mates to grooming and proximity maintenance within pairs and to the territorial defense in seven titi pairs. Females were more active in controlling proximity, making most of the approaches and leaves within pairs. Females also groomed males more than the males groomed them, and there was a prominent difference in grooming reciprocity between the periods before and after infant birth. Before infant birth, grooming was roughly reciprocal, but during the infant dependency period, females groomed males more than twice as much 
as males groomed them. This skew resulted from males considerably reducing the amount of grooming directed at females after infant birth, while the females maintained their grooming effort almost at the same level. Males, on the other hand, contributed more than females to the territorial defense. They participated in more intergroup encounters than females and were more active during these encounters, chasing the opponents and calling more often than did females. Together, these results suggest that females contribute to the maintenance of proximity and affiliation within pairs "in exchange" for services provided by males, such as infant care and territorial defense. This effect is especially pronounced during the infant dependency period, when male services are most needed.

\section{Biparental care and its effects on the caring adults}

After showing that infant presence affected grooming reciprocity within pairs, I further investigated the effects of infant care on activity and social relationships of caring adults. The analysis of activity budgets and diet composition of adults in seven titi groups showed that the effects of infant care were mostly opposite in females and males. While females fed more, consumed more arthropods and rested less, males fed less, consumed fewer arthropods and rested more after the infant birth than before. For both sexes, social time dropped considerably after the infant birth. Both the female strategy of "eating more" and the male strategy of "doing less" presumably helped to compensate for the energetic costs of infant care. The opposing effects were likely attributed to the increased requirements for proteinrich food (arthropods) in lactating females, on one hand, and by incompatibility of infant carrying with effective arthropod foraging and vigilance in carrying males, on the other hand. Further, I examined the trade-offs between activity categories in lactating females to see which activity was used to offset the increased feeding time. I showed that lactating females increased their feeding time at the expense of mainly resting and, to a lesser degree, social time. Despite the general decrease in social time, females conserved the amount of grooming directed at their pair mates, indicating the importance of male services for them.

\section{Anti-predator behaviors}

Lastly, I documented the encounters of titis with their potential predators. These data were only anecdotal, as predator encounters cannot be predicted. Nevertheless, in 4 out of 10 encounters males were more active than females in anti-predator behaviors, while the reverse was never observed. These findings are in line with predominantly male participation in territorial defense. Additionally, I documented mobbing behavior, not reported for $P$. cupreus before, and other active anti-predator reactions rarely observed in titis. Although not directly related to the aim of this thesis, these observations add to our knowledge about titis. 


\subsection{Synthesis}

Central for our understanding of the social system of coppery titis is their monogamous mating system and the roles that females and males play in its maintenance. Here, I will first discuss the potential costs and benefits of genetic monogamy to female and male titis and its proximate influences. I will then summarize the contributions of females and males to the maintenance of different "monogamy package" components and the factors that are most important for the maintenance of titis' social system, in particular, strong pair bond, high level of male care and unconstrained dispersal. Lastly, I will discuss the implications my findings may have for our understanding of the evolution of monogamy.

\section{Costs and benefits of genetic monogamy}

Both males and females face trade-offs between staying faithful to their social partner and engaging in extra-pair copulations (EPC) (Petrie \& Kempenaers 1998). The benefits of EPC are more obvious for males, because this way they might increase their reproductive success while avoiding the costs of parental care (Clutton-Brock \& Parker 1992). However, the benefits of EPC to females are increasingly recognized, too (reviewed in, e.g., Jennions and Petrie, 2000; Kempenaers, 2007). They include paternity confusion to prevent infanticide, insurance against infertility of their social mate and indirect (genetic) benefits, expressed as the increased genetic quality of offspring (Petrie \& Kempenaers 1998; Jennions \& Petrie 2000; Wolff \& MacDonald 2004; Sheldon 1994). Considering all these potential benefits of EPC, why do titis stay mostly genetically monogamous?

First, there are costs to seeking EPC. For both sexes, these costs include aggression from samesex adults and the increased risk of acquiring sexually transmitted diseases, as evidenced by higher prevalence of sexually transmitted pathogens in more promiscuous species of birds and mammals (Petrie \& Kempenaers 1998; Poiani \& Wilks 2000; Nunn \& Altizer 2006; MacManes 2011). For males, there is additionally a trade-off between time spent searching for EPC and guarding their mates from other males (Birkhead \& Biggins 1987; Stutchbury \& Morton 1995). And for females, EPC might come at a risk that a cuckolded male reduces or withholds infant care, as shown in many bird species (e.g., Dixon et al., 1994; Osorio-Beristain and Drummond, 2001).

The link between male care and paternity, however, requires that a male is able to assess his paternity. This can be done by recognizing relatedness between him and offspring using olfactory, visual, or acoustic cues (phenotypic matching: Kazem and Widdig, 2013; Mateo, 2017; Pfefferle et al., 2013) or simply by seeing his social mate engaging in copulations with other males (Whittingham \& Dunn 2001). In titis, there have been no studies on kin 
recognition. But the high degree of proximity and coordination between pair mates should make it easy for males to detect any extra-pair activities of their mates.

It is unclear, however, if male titis would stop caring for infants even if they knew they were unrelated. One case of adoption was reported for $C$. nigrifrons, where an infant was moved, for unknown reasons, from his natal group to another group that already had an infant. A male provided the same amount of care to both infants and the female nursed both of them. Both infants survived and could not be differentiated in terms of behavior or their relationships with the other groups members (Cäsar \& Young 2008). There is also anecdotal evidence that after adult replacements in P. toppini, the relationships between group members quickly stabilized and did not differ from those before the replacement (Rodman \& Bossuyt 2007). In our study, there were no cases of male replacements and only one inferred case of female replacement. In this case, too, there was nothing unusual in the relationships between the group members. In Azara's owl monkeys, Aoutus azarae, who are very similar to titis in all aspects of their social system, including social and genetic monogamy and very high level of male care, males were shown to care for unrelated infants as much as they do for their own offspring, and replacements of putative fathers by new males did not have any negative effects on infant survival (Huck \& Fernandez-Duque 2012a, 2012b). All these observations suggest that in titis, the risk that a cuckolded male would withhold infant care is probably quite low.

\section{Genetic monogamy and population density}

In addition to the costs of EPC, genetic monogamy might be simply related to the lack of possibilities for extra-pair activities. If the animals are scarcely distributed in space, the encounters between them and, consequently, EPC are expected to be less likely (Westneat 1990). Indeed, the positive relationship between population density and EPP rates was demonstrated in many bird studies (e.g., Brouwer et al., 2017; Westneat and Sherman,1997). In domestic cats, Felis catus, mating system was shown to depend on the population density, influenced, in turn, by different environmental conditions (Say et al. 1999). At high population densities in urban environments, cats are promiscuous, while at intermediate densities in rural areas, males are able to monopolize several females, which results in polygynous mating system (Say et al. 1999). Finally, at a very low density, such as in a population of sub-Antarctic Kerguelen Island, cats are genetically monogamous, as it is not beneficial for males to try searching for and defending access to more than one female (Say et al. 2002). Similar pattern was demonstrated for Eurasian beavers, Castor fiber, where a lower density population living in a habitat with harsher climate was found to be genetically monogamous, while a higher density population from an area with milder climate had a low proportion of EPP (Nimje et al. 2019; Syrůčková et al. 2015). 
The population density at our study site was relatively low (34 individuals $/ \mathrm{km}^{2}$, lying within the average range of densities reported in the behavioral studies ${ }^{1}$ of undisturbed populations of titis: (Fernandez-Duque et al. 2020; Dacier et al. 2011; Bicca-Marques \& Heymann 2013), likely limiting opportunities for EPC. Notably, the only published record of EPC in titis comes from a population of Plecturocebus ornatus (previously Callicebus moloch) with exceptionally high density of 406 individuals $/ \mathrm{km}^{2}$ (Mason 1966). At this study site, nine family groups (28 animals in total) inhabited a single isolated forest fragment of only 6.9 ha. For comparison, the average size of the home range of one group at our study site was 7.2 ha, larger than the whole fragment inhabited by nine groups of P. ornatus. In P. ornatus study, EPC and aggression between pair mates, as well as fights during the intergroup encounters, were frequently observed. At our study site, we observed neither of these behaviors.

It should be mentioned that a preliminary analyses in a population of $P$. discolor from an undisturbed habitat reported three cases of EPP in a sample size of 16 offspring, although these data has not been published yet (Van Belle et al., 2016b, conference abstract). The density of this population (57 individuals $/ \mathrm{km}^{2}$ ) was higher than that of our study population, the home ranges were smaller, averaging 5.0 ha (2.0-8.5), and the percentage of home range overlap between the neighboring groups was larger, 4.8\% (0-13) (compared to 1.4\% (0-4.7) in our study population) (Fernandez-Duque and Fiore, 2020, unpublished data). Although these differences are not large, it is possible that higher population density and higher home range overlap could account for the higher EPP rate in P. discolor.

\section{Population density and deviations from pair-living}

Another mechanism linking population density with the EPP rates is natal dispersal. In a population with high density, offspring might delay dispersal while waiting for the territorial vacancies to become available (Bowler \& Benton 2005; Matthysen 2005; Kokko \& Ekman 2002; Emlen 1982). Delayed dispersal in saturated habitats has been demonstrated in, e.g., Eurasian beavers, white-handed gibbons, Hylobates lar, and fork-marked lemurs, Phaner furcifer (Mayer et al. 2017b; Schülke 2003; Brockelman et al. 1998; Mayer et al. 2017a, 2020). In some cases, delayed dispersal might even be beneficial for young animals, giving them extra time to mature and increasing their future chances to acquire a better territory and/or partner, as shown, e.g., in Eurasian beavers and Siberian jays, Perisoreus infaustus (Mayer et al. 2017a; Ekman et al. 1999). But most often, delayed dispersal in pair-living animals also

\footnotetext{
${ }^{1}$ Traditional population census methods, e.g., line transects, were shown to strongly underestimate population densities of elusive and cryptic species, such as titis (Dacier et al. 2011). For this reason, for the comparisons here, I use population density estimations based on the home range sizes obtained in behavioral studies; the same method was sued to estimate the population density at our study site. This method was shown to be the most reliable for estimating population densities in diurnal primates (Hassel-Finnegan et al. 2008).
} 
means delayed reproduction, because offspring will usually not mate with their parents to avoid inbreeding (Emlen 1982; Bowler \& Benton 2005).

This might change, however, if an opposite-sex parent is replaced by an unrelated adult, providing the offspring with the breeding opportunities within their natal group (Emlen 1982). If adults then tolerate the offspring breeding in their natal group, deviations from pairliving might occur, as shown, e.g., in sakis, Pithecia spp. and swift foxes, Vulpex velox (Kitchen et al. 2006; Norconk \& Setz 2013; Van Belle, Fernandez-Duque, et al. 2016; Porter et al. 2017). The link between adult replacements and delayed dispersal was even demonstrated experimentally in pair-living white-footed mice, Peromyscus leucopus, where the removal of the opposite-sex parent lead to the delayed offspring dispersal (Wolff 1992). However, replacements do not always have this effect. For example, in Azara's owl monkeys, offspring never reproduced in their natal groups and did not delay dispersal after the replacement of same-sex parent (Huck \& Fernandez-Duque 2012a; Corley et al. 2017). Rather, long-term data indicated increased local mate and resource competition between offspring and same-sex parent after replacements, indicating that in owl monkeys, offspring were unlikely to benefit from delayed dispersal even after parent replacement. This was supported by a case report of a young male who delayed dispersal after the replacement of adult female in his group. After trying to copulate with his step-mother, the male was vigorously attacked and evicted by his putative father (Huck \& Fernandez-Duque 2012a).

It is unclear if deviations from pair-living occur in titis. In our study population, group size was never more than five, and subadults dispersed shortly before or after the birth of a new infant. There are a few reports of groups with more than two adult-size individuals in other titis species, most of them from the studies in isolated forest fragments or larger fragments with high population density (Felton et al. 2006; Cäsar et al. 2012; Bicca-Marques et al. 2002). At the same time, a survey of 43 P. ornatus groups living in forest fragments with high population densities did not find groups with more than two adults (Wagner et al. 2009), while a Callicebus personatus group with three adult-sized animals was reported in an undisturbed habitat (Price \& Piedade 2001). These findings suggest that dispersal can be additionally mediated by factors other than population density. In any case, in the absence of genetic and long-term demographic data, it is not possible to tell if the extra adult-size individuals from these reports were breeding adults or non-breeding offspring. There is only one detailed observation of delayed dispersal in titis, in which a young $P$. discolor male returned to his natal group one month after dispersal and remained there for almost two years beyond the age of adulthood (Van Belle, Fernandez-Duque, et al. 2016). Notably, this happened after the immigration of an unrelated female, and the male was seen mounting the 
female. However, he did not copulate with her and eventually dispersed. None of the offspring observed in this study (three groups monitored for 12 years) bred in their natal groups, either (Van Belle, Fernandez-Duque, et al. 2016). These observations suggest that in some cases offspring in titis may delay dispersal but are not very likely to reproduce in their natal groups.

\section{Dispersal and floaters}

When individuals cannot reproduce in their natal groups, they have three options available to them (Greenwood 1980; Kokko \& Ekman 2002; Emlen 1982). First, they can stay as nonreproducing helpers, which might eventually lead to the formation of cooperatively breeding groups. Second, they can move to a territory adjacent to their natal home range if it happens to be vacant, as was observed in this study (Chapter 3). Finally, they can disperse and become floaters in search of territories.

Floaters, solitary non-territorial individuals ranging over a wide area after having dispersed from their natal groups, have been reported for many mammal species, including titis' closest pair-living relatives, sakis and owl monkeys (Fernandez-Duque \& Huck 2013; Huck \& Fernandez-Duque 2012a; Thompson \& Norconk 2011; Di Fiore et al. 2007). To reproduce, floaters can either find a vacant territory, fill a vacancy left by a death of a same-sex resident animal or forcefully replace the same-sex resident animal (Emlen 1982; Kokko \& Ekman 2002). In Azara's owl monkeys, where floaters have been extensively studied, they were shown to frequently replace resident individuals, often by challenging and evicting them from their groups (Fernandez-Duque \& Huck 2013; Huck \& Fernandez-Duque 2012a). Alternatively, floaters may copulate with residents without trying to acquire a territory, but this seems to happen much less frequently than one might intuitively expect. Most studies on mammals and birds indicate that extra-pair offspring are usually sired by neighboring individuals, not floaters (e.g., Barelli et al., 2013; Nimje et al., 2019; Petrie \& Kempenaers, 1998). Although, some evidence indicate that male floaters sometimes sire offspring, too (e.g., in alpine marmots, Marmota marmota: Cohas, Yoccoz, Da Silva, Goossens, \& Allainé, 2006; golden-cheecked gibbons, Nomascus gabriellae: Kenyon, Roos, Binh, \& Chivers, 2011). In birds, female floaters were also shown to sometimes reproduce via brood parasitism (e.g., in European starlings, Sturnus vulgaris: Sandell \& Diemer, 1999). However, in mammals, female floaters probably would not be able to raise offspring without a territory. Therefore, in most cases, floaters will have to become residents to be able to reproduce.

In titis, only anecdotal reports of floaters exist (Lawrence 2007; Rodman \& Bossuyt 2007; Bossuyt 2002; Palacios et al. 1997; Wright 2013). In a 12-years long study on P. discolor, several female replacements and one possible male replacement were observed; in all cases, the breeding positions vacated after the disappearance of adults were promptly occupied by 
immigrants (Van Belle, Fernandez-Duque, et al. 2016). In contrast to the owl monkeys, no evictions by intruding floaters were observed. In our study population, there was only one case of inferred female replacement, and we never noticed any intruders. However, after the dispersal of a young male from his natal home range to an adjacent area, he was seen with a female mate already on the next day, suggesting that there must be some individuals floating around and waiting for the breeding opportunities to emerge. Solitary individuals can be very difficult to detect in the field (Fernandez-Duque \& Huck 2013), and probably even more so in animals as shy and secretive as titis. Moreover, in Azara's owl monkeys, floaters were shown to stay at the edge of groups' home ranges, avoiding the core areas (Huck \& Fernandez-Duque 2017). If floaters in titis behave the same way, it would make them even more difficult to notice. Therefore, it seems likely that there are at least some floaters in titi populations. It remains unclear why, despite all the similarities between titis and owl monkeys, no evictions by floaters have been reported in titis, not even in the 12-years long study. Possibly, evictions happen so infrequently that more data will be needed to detect them.

Here, it is important to note two points. First, if floaters exist in a population, density values obtained from home range sizes, including the value reported in this study and in other studies used for comparison here, are likely to be underestimated. Second, adult replacements by floaters (or any other individuals) can create groups that do not consist of biological parents and their offspring, leading to the apparent deviations from genetic monogamy even in the absence of EPC. In my study, one paternity remained unassigned. Assuming it was not just a product of genotyping error, it could result either from EPC or from male replacement after the birth of the offspring. Therefore, when examining mating patterns of titis or other pair-living animals, it is crucial to have long-term demographic data to be able to distinguish between cases of EPP and serial monogamy.

\section{Inbreeding avoidance and dispersal}

One of the ultimate reasons for dispersal, in addition to the avoidance of mate and resource competition, is inbreeding avoidance (reviewed in Bowler and Benton, 2005). In our study population, dispersal appeared to be sufficient to avoid inbreeding. This was indicated by the low mean relatedness between the pair mates despite the lack of evidence for active inbreeding avoidance via mate choice. Low relatedness between mates in the absence of active inbreeding avoidance has been demonstrated in many other mammals and birds (e.g., Hansson et al. 2007; Geffen et al. 2011). In fact, very few of the numerous studies on mate choice in birds have documented active inbreeding avoidance via mate choice (reviewed in Jamieson et al., 2009). It has been suggested that when dispersal results in low probability of encountering close kin, active inbreeding avoidance might not be necessary (Jamieson et al. 
2009; Hansson et al. 2007). In this case, kin discrimination mechanisms will not evolve, and low inbreeding levels that occasionally occur in such systems will be tolerated. For example, in grey wolves, Canis lupus, and arctic foxes, Vulpes lagopus, mating outside of natal groups was random with regard to relatedness; importantly, it was the case not only in outbred populations with low kin encounter rate, but also in inbred populations (Geffen et al. 2011). Since historically grey wolf and arctic fox populations were large and spanned vast areas, kin encounter rates and inbreeding risk were likely low. Consequently, the mechanisms of inbreeding avoidance by kin discrimination did not evolve, explaining why individuals still mate randomly in respect to relatedness in populations that recently became inbred (Geffen et al. 2011). In some cases, the lack of mechanisms for inbreeding avoidance via kin discrimination can lead to very high levels of inbreeding if the populations become fragmented or isolated, as shown, for example, in koalas, Phascolarctos cinereus, and bighorn sheep, Ovis canadensis (Rioux-Paquette et al. 2010; Schultz et al. 2020; Jamieson et al. 2009). Our study population was neither fragmented nor isolated, which presumably helped to keep the kin encounters rate at a low level and ensured passive inbreeding avoidance.

Additionally, inbreeding may occur not because it cannot be avoided, but because the costs of being choosy are higher than the costs of inbreeding itself (Jamieson et al. 2009). For example, in pair-living or cooperatively breeding species, acquiring a territory may be more important than inbreeding avoidance, and in species with low survival rates the costs of foregoing matings to avoid inbreeding may be higher than the costs of inbreeding itself (Keller \& Arcese 1998; Jamieson et al. 2009). This trade-off may promote random mating even when kin encounter rates are high. In our study population, one pair consisted of second-degree kin, suggesting that low inbreeding levels might be tolerated in titis. However, the data was not sufficient to determine whether it was a result of the trade-off between choice for a partner vs. choice for a territory or a consequence of the lack of kin discrimination mechanisms. To see if titis indeed lack mechanisms for inbreeding avoidance via kin discrimination or if they only mate randomly in undisturbed populations, it will be necessary to compare mating patterns and inbreeding levels in continuous vs. fragmented or isolated populations.

\section{Genetic monogamy, pair bond and mate guarding}

In addition to population-level factors, EPP rates are influenced at the more proximate level by the relationships between pair mates, or pair bonds. While a strict definition of the pair bond requires that it is characterized by behavioral, emotional, and endocrinological characteristics, such as physiological distress upon separation (Hinde et al. 2016), in practice such confirmation is rarely possible. In the field studies, pair bond is usually assessed by the degree of spatial cohesiveness, coordination and affiliation of the pair mates or by the 
exchange of services between mates (Fuentes 2002; Hinde 1983; van Hooff \& van Schaik 1992). A high degree of cohesiveness and coordination between pair mates, or strong pair bond, is expected to facilitate mate guarding and thus limit the opportunities for EPC, keeping EPP at low levels (Brotherton \& Komers 2003; Huck et al. 2014). Especially successful mate guarding can lead to strict genetic monogamy, as shown, for example, in Kirk's dik-diks, Madoqua kirkii (Brotherton \& Komers 2003; Brotherton et al. 1997).

Mate guarding is most commonly defined as a male behavior preventing a receptive female from copulating with other males by maintaining close proximity with her (Birkhead 1995; Alberts et al. 1996), although the term has been used differently by other authors (reviewed in Manson, 1997) ${ }^{2}$. Thus, strictly speaking, mate guarding should only occur during the period of female fertility, i.e., around ovulation. My dataset was not sufficient to determine if male titis exhibit mate guarding in this strict sense, as the time of ovulation of the study females was not known. In three groups (Groups 1, 4 and 5), the males closely followed their mates on the same days when they were seen copulating. This following behavior was also reported in captive P. cupreus males (Anzenberger 1988). However, the females on these days were closely following their mates, too, and soliciting copulations from them. On several occasions in Group 5, the male was observed to withdraw from the soliciting female and leave. Therefore, it was difficult to determine if the males were specifically following and guarding the females or if both pair mates were equally interested in maintaining proximity. In many mammals, mate guarding additionally include more aggressive behaviors. For example, in Kirk's dik-diks, males vigorously fight with the male intruders and aggressively chase the females back to their home ranges if they cross the border (Brotherton \& Komers 2003). In this study, such behaviors were never observed. However, anecdotal evidence from other titi species indicate that agonistic interactions can occur if females actually try to engage in EPC. In P. ornatus, a male was observed to interrupt female's copulations with a neighboring male and to restrain her when she tried to move away, and in P. toppini, a male repeatedly attacked a female when she was trying to copulate with a neighboring male (Lawrence 2007; Mason

\footnotetext{
${ }^{2}$ An interesting question is whether females can guard males, too. In pair-living animals, males are expected to guard their mates more than females because of the higher costs of pair mate infidelity for males (Westneat \& Stewart 2003; Hosken et al. 2009; Birkhead \& Biggins 1987; Trivers 1972). A cuckolded male might lose the entire reproductive season or waste costly care at unrelated young, while a female does not risk much given that a male continues to provide infant care or other services. Indeed, examples of female mate guarding are rare and in most cases, seem to be a result of females trying to monopolize resources or infant care available to their young (Hosken et al. 2009). For example, burying beetles, Nicrophorus defodiens, lay eggs in carcasses, and the resident female prevents her pair mate from attracting additional females to carcasses, because their presence would reduce resources and male care available for her larvae (Eggert \& Sakaluk 1995). Another example is a European starling, Sturnus vulgaris, where females interrupt the attempts of their pair mates to attract extra females because monogamously mated males provide significantly more help to females in incubating eggs and feeding nestlings than polygynous males (Eens \& Pinxten 1995).
} 
1966). In both these studies, the competing males also engaged in contact aggression (physical fights) during the intergroup encounters, which otherwise happens very rarely in titis and was never observed in this study (Bossuyt 2002; Wright 1984; Robinson 1979).

Apart from mate guarding in the strict sense, males in some species seem to "guard" the females also beyond their fertility period. For example, in Kirk's dik-diks, males maintain close proximity with females and chase them back to their home ranges not only during estrus, but year-round (Brotherton et al.1997). Presumably, this way the males advertise the paired status of the females, reducing the risk of male territorial challenges, and maintain pair integrity, preventing the females from switching territories (Brotherton et al. 1997). Existing evidence suggests that in titis, too, males maintain pair integrity beyond periods of female fertility. In captivity, P. cupreus males consistently showed more agitation and distress than females in the presence of same-sex strangers (Anzenberger 1988; Cubicciotti \& Mason 1978; Mendoza \& Mason 1986; Fernandez-Duque et al. 2000). In field studies, males participated in the intergroup encounters more often and more actively than females and were the first to initiate calling in response to simulated (via playbacks) intruders (Lawrence, 2007; Robinson, 1981; Wright, 1984; this study). Finally, my observations suggest that males might be unwilling to lose track of females, as males usually showed more signs of distress when separated from their mates than females. These observations, however, were only qualitative and will have to be substantiated by quantitative data collection. Additionally, both in this study and in P. toppini, males followed the females more often than otherwise during traveling (69\% of recorded group travel sequences in this study, unpublished datal; Wright, 1984), which might also indicate that males try to keep females in sight.

Summing up, the evidence for mate guarding in the strict sense remains inconclusive in titis. However, the available data suggest that males maintain the integrity of pairs via territorial defense year-round, not only during the female fertile periods.

\section{Territorial defense, mate guarding and resource defence}

Mate guarding and the maintenance of pair integrity are not the only functions of territorial defense. Like pair bond, territorial defense can have several purposes, and these purposes are not necessarily the same for females and males. Early hypotheses suggested that females should mainly defend resources, while males should defend mates ("food resource defense" and "mate defense" hypotheses). This was based on the consideration that female reproductive success is mainly constrained by the access to resources, while male reproductive success is constrained by the access to mates (Emlen and Oring, 1977; van Schaik and Dunbar, 1990; Trivers, 1972; Wrangham, 1980). However, more recently, it was suggested that these functions of territorial defense do not have to be mutually exclusive 
(Fashing 2001). While driving away competitors as part of mate guarding, males may at the same the same time defend the territory with resources ("direct mate defense" hypothesis). Alternatively, when defending a territory with resources, a male might simultaneously guard his mate ("indirect mate defense" hypothesis) (Fashing 2001). In both scenarios, females might also participate in resource defense, albeit at lower rates than males. Both hypotheses predict that males will display more territorial behaviors than females, especially when females are fertile. The "indirect mate defense" hypothesis additionally predicts that males will display more territorial behaviors in the intensively used parts of their home range and will defend their pair mates year-round, not only when they are fertile (Fashing 2001).

My dataset was not sufficient to systematically test these hypotheses. Intergroup encounters were too rare, with less than one encounter per week in one group on average; two of the seven observed groups did not engage in the encounters at all during the study period. In addition, female fertile periods were unknown. Still, my observations, combined with the findings of other studies on titis, provide some support for the "indirect mate defense" hypothesis. First, in our study population and in other titi species, males were more active than females in the intergroup encounters, and not only when females were receptive, but also when they were gestating or lactating (Lawrence 2007; Robinson 1981; Caselli et al. 2014). Second, in playback studies in P. toppini, P. discolor and C. nigrifrons, males displayed more territorial behaviors in the intensively used parts of the home range, as well as when simulated intruders were closer to their mates (Lawrence 2007; Caselli et al. 2015; Robinson 1981). In contrast, females reacted stronger to the playbacks in the intensively used parts of the home range, while their reaction to the simulated intruders being close to their mates was weaker than that of males (Lawrence 2007; Robinson 1981). Combined with the evidence for stronger male than female reaction to the same-sex intruders in captive studies, these findings suggest that in titis, males engage in indirect mate defense via resource defense, while female contribute to some extent to the resource defense. Similar pattern was demonstrated in sakis, the closest pair-living relatives of titis (Thompson et al. 2012).

\section{Biparental care and monogamy}

The last important component of "monogamy package" in titis is biparental care. The exceptionally high level of male care in titis raises the question of why infant care is beneficial to males. It has been suggested that males may benefit from infant care in at least three ways. First, they might get direct benefits through enhanced survival of their infants ("genetic advantage" hypothesis: Wuensch, 1985). Second, male care may partly relieve a female from the energetic costs of infant care, allowing her to produce offspring at a higher rate or of better condition ("maternal relief" hypothesis: Woodroffe and Vincent, 1994). Finally, a male might 
provide infant care in exchange for some benefits from a female, such as matings ("mating strategy" hypothesis: Smuts and Gubernick, 1992).

The evaluation of "genetic advantage" and "maternal relief" hypotheses requires the comparison of infant survival in groups with and without male caretaker. In titis, such groups have never been reported and would probably not exist for long, as any breeding vacancies are likely to be filled quickly by new adults. But there is some indirect evidence to evaluate these hypotheses. First, in all three cases of twinning reported in the wild, one of the twins did not survive beyond the age of 6 months (Knogge \& Heymann 1995; Lawrence 2007; de Santana et al. 2014). Even in captivity, one of the infants died on the day of birth in both reported cases (Valeggia et al. 1999). Second, in the reported case of adoption in C. nigrifrons, adults cared for two infants of the same age at the same time, and both infants survived; however, the female did not reproduce the next year, suggesting that even if the twins can be successfully raised in some cases, there will be reproductive costs for females (Cäsar \& Young 2008). These observations suggest that females might not be able to invest more in the offspring than they normally do. This indicates the importance of male care for infants and mothers, supporting both "genetic advantage" and "maternal relief" hypotheses.

In contrast to the "genetic advantage" hypothesis, "maternal relief" and "mating strategy" hypotheses predict that males should care not only for their own offspring, but for unrelated young, too. In titis, there is no systematic data on this, but the case of adoption mentioned above, combined with anecdotal data on male replacements, suggest that males may care for unrelated infants. However, it is difficult to distinguish between these two hypotheses without infant survival data and maternal energy budgets in groups with and without male care. Existing data provide indirect support for both hypotheses. First, inter-birth intervals are shorter after the death of infants both in the wild and in captivity, indicating that females can return to reproduction faster when relieved of the costs of infant care and thus supporting the "maternal relief" hypothesis (Van Belle, Fernandez-Duque, et al. 2016; Valeggia et al. 1999). In our study population, the majority of births occurred between October and February, but one was recorded in June, indicating that births are not strictly seasonal. Second, changes in the activity budgets of male caretakers suggest energetic costs of infant carrying. Therefore, by taking charge of infant carrying, males relieve the females from these costs. Given that older siblings hardly ever carry infants (there is only one field report of a juvenile briefly carrying a newborn in P. cupreus: Terrones Ruiz et al., 2004), females probably would not be able to delegate infant care to anyone else. Finally, in addition to direct infant care, male provide territorial and antipredator defense, allowing the mothers to spend more time foraging. These findings support the "maternal relief" hypothesis. However, there is also 
some evidence supporting the "mating strategy" hypothesis. In this study, females groomed males more than vice versa during the infant dependency periods, suggesting that infant care might make males more attractive to females. Given that female titis are the same size as males (Heymann et al., 2012; Norconk, 2011; Smith and Jungers, 1997), females probably can control matings by offering or withholding them.

Summing up, while it is difficult to distinguish between "maternal relief" and "mating strategy" hypotheses, the existing data suggest that in titis, both males and females benefit from male care. This likely helps to maintain social and genetic monogamy by creating a positive feedback between the increased female fecundity and male care, as suggested by a recent comparative phylogenetic analysis on distribution of male care in mammals (West \& Capellini 2016).

\section{Implications for the evolution of monogamy in New World monkeys}

The highest levels of male care within non-human primates are found in several taxa of the New World monkeys (platyrrhines), namely titis, owl monkeys and callitrichines (tamarins and marmosets) (Huck \& Fernandez-Duque 2012b). Furthermore, platyrrhines have very high proportion of pair-living taxa compared to other simian primates (van Schaik \& Kappeler 2003; Lukas \& Clutton-Brock 2013). Titis and owl monkeys (Aotus spp.) are consistently pairliving, and sakis (Pithecia spp.) are mainly pair-living (Fernandez-Duque et al. 2020). Additionally, pair-living can occur in callitrichines, although it is not their modal social organization (Heymann 2000; Garber et al. 2016).

However, the taxa with pair-living and male care do not form monophyletic groups within the platyrrhines. Titis and sakis, together with group-living uacaris, belong to the family Pitheciidae; owl monkeys and callitrichines belong to the family Cebidae that also includes group-living capuchins and squirrel monkeys, and Cebidae forms sister group to family Atelidae that consists exclusively of group-living taxa (spider monkeys, woolly monkeys and muriquis). This raises a question about the evolutionary history of pair-living and male care within the platyrrhines. In particular, a matter of ongoing debate is the position of owl monkeys (Rosenberger \& Marcelo 2013; Bjarnason et al. 2017). Titis and owl monkeys are remarkably similar both morphologically and behaviorally: they are socially monogamous, sexually monomorphic, territorial, form strong pair bonds and exhibit high level of male care (Fernandez-Duque and Fiore, 2020; Huck and Fernandez-Duque, 2012b; Kinzey, 1997; Wright, 1984). Despite all these similarities, molecular phylogenetic analyses consistently group owl monkeys with the Cebidae rather than the Pitheciidae to which the titis belong (Schneider \& Sampaio 2015; Perelman et al. 2011; Valencia et al. 2019). 
Fossil and molecular data indicate that platyrrhines originated in Africa around 43 million years ago and dispersed into South America during the Eocene, probably by rafting on budding forest islets (Silvestro et al. 2019; Oliveira et al. 2009). The ancestral state reconstructions suggest that the ancestor of platyrrhines was small-bodied, the size of a tamarin (less than $1 \mathrm{~kg}$ ), which was probably a key factor enabling the survival of dispersers on floating islets, since small-bodied animals would require relatively little resources (Silvestro et al. 2019). The phylogenetic analysis of sociality in primates further indicated that this platyrrhine ancestor was likely pair-living (Kappeler \& Pozzi 2019). Pair living could probably also facilitate the survival of animals on floating islets as they did not need as much resources as larger groups. The pair-living ancestry of platyrrhines is also in accordance with the basal position of Pitheciidae (where the majority of species are pair-living), as well as with the basal position of pair-living titis within Pitheciidae (Perelman et al. 2011; Valencia et al. 2019). In agreement with the idea of pair living as a stepping stone towards the evolution of group living from solitary ancestors (Kappeler \& Pozzi 2019), the next group to split off after Pitheciidae was Cebidae, consisting of pair-living owl monkeys, occasionally pair-living callitrichines and group-living squirrel and capuchin monkeys. Finally, the youngest radiation within the platyrrhines is the family Atelidae, consisting exclusively of large-bodied groupliving taxa (Perelman et al. 2011; Valencia et al. 2019).

The pair-living ancestry of platyrrhines might explain why titis and owl monkeys are so similar despite their distant positions on the phylogenetic trees. In agreement with this idea, a recent phylogenetic analysis indicated that morphological similarities between these two taxa are likely a mix of the retention of ancestral platyrrhine traits and convergence (Bjarnason et al. 2017). The ancestral state of small body size suggested for platyrrhines would then mean that titis and owl monkeys underwent convergent evolution towards the medium body size, while the callitrichines kept small sizes throughout their evolutionary history (Silvestro et al. 2019). This scenario would also be consistent with both alternative positions of owl monkey within the Cebidae suggested by different phylogenetic analyses: a basal position and a position as a sister group to callitrichines (Valencia et al. 2019).

A promising approach for the understanding of the distribution of monogamy in the platyrrhines is offered by the studies of the neuropeptide hormone oxytocin and its oxytocin receptor protein (OXTR) that are known to be involved in the expression of social behaviors and parental care in mammals (Vargas-Pinilla et al. 2015). It was shown that in addition to the conserved form of oxytocin shared by all placental mammals, platyrrhines have four unique oxytocin variants and substantially more mutations in the OXTR coding sequence than other primates (Babb et al. 2015; Vargas-Pinilla et al. 2015). One of these oxytocin variants is 
unique for the Cebidae taxa, providing additional support for the grouping of owl monkeys within this family rather than within Pitheciidae (Lee et al. 2011). Given the high proportion of pair-living taxa in platyrrhines in comparison to other mammals, these findings suggest the link between the oxytocin pathway and the evolution of monogamy in primates. However, no specific form of OXTR was found that would discretely cluster with socially monogamous primate taxa, indicating that variation in oxytocin and its receptor alone does not explain phylogenetic patterning of monogamy in primates (Babb et al. 2015). This suggests that social monogamy and its correlates might be maintained and/or have evolved through different pathways in different taxa, not exclusively through the oxytocin pathway.

While all comparative analyses in mammals agree that male care was most likely a consequence rather than a cause of social and/or sexual monogamy (Lukas \& Clutton-Brock 2013; Komers \& Brotherton 1997; Opie et al. 2013), its evolutionary history in platyrrhines remains unclear. Despite being the closest relatives of titis, sakis do not exhibit male care (Fernandez-Duque et al. 2013; Norconk 2011). One possible explanation for this is the larger body size of sakis compared to titis and owl monkeys. In smaller-bodied taxa, male care might be more important because it reduces the metabolic costs of raising a relatively large offspring to the female (Fernandez-Duque et al. 2013). Alternatively, it was suggested that the absence of male care in sakis is the result of more pronounced sexual dimorphism, higher paternity uncertainty and more male-biased sex ratio compared to titis and owl monkeys (Norconk 2006; Huck \& Fernandez-Duque 2012b). All these factors are expected to lead to more "classical" sex roles, with stronger sexual selection in males than females, which, in turn, should favor female-biased infant care (Kokko \& Jennions 2008). By contrast, equal sex ratio, sexual monomorphism and high paternity certainty, as observed in owl monkeys, should favor the evolution of egalitarian sex roles with regards to infant care (FernandezDuque \& Huck 2013; Huck \& Fernandez-Duque 2012b; Kokko \& Jennions 2008). In titis, there are no long-term data on the sex ratios. However, given their sexual monomorphism and high paternity certainty demonstrated in this study, it seems reasonable to predict equal sex ratios in titis, too. This should favor egalitarian sex roles with regard to infant care, which, in turn, will reinforce social and genetic monogamy by providing benefits to both sexes. 


\section{Conclusions}

In this study, I showed that in coppery titis, social monogamy is translated into genetic monogamy (Chapter 3). My findings, together with evidence from other studies on titis, indicate that three factors are important for maintaining social and genetic monogamy in titis: strong pair bond, high level of male care and possibilities for unconstrained dispersal. The analyses of pair-bond maintenance mechanisms demonstrated that the females contribute more to the maintenance of affiliation and proximity between the pair mates, while the males contribute to the maintenance of pair integrity via territorial defense (Chapter 4). Both of these contributions likely limit the probability of extra-pair copulations and thus promote genetic monogamy. Hight level of male care for infants further helps to maintain social and genetic monogamy in titis. By relieving the female from some costs of infant care, male care provides benefits for both sexes. On one hand, it allows the females to forage more to compensate for the energetic costs of lactating, and on the other hand, it likely provides mating advantages to males by making them more attractive to the females, as suggested by female-based grooming within pairs during the periods of infant care (Chapter 5). Finally, both social and genetic monogamy would probably be compromised without the unconstrained dispersal possibilities available in an undisturbed habitat. Unconstrained dispersal in our study population likely allowed the offspring to search for breeding opportunities outside of their natal groups, preventing the deviations from pair-living which might result from delayed offspring dispersal. Additionally, unconstrained dispersal served to prevent inbreeding even in the absence of active inbreeding avoidance via mate choice, thus rendering extra-pair copulations unnecessary (Chapter 3). Summing up, these findings demonstrate that social and genetic monogamy can be influenced not only by the components of a species' social system, such as pair bond and male care, but also by extrinsic factors, such as habitat characteristics ensuring unconstrained dispersal. While unconstrained dispersal helps to maintain social monogamy by preventing deviations from pair living, strong pair bond and male care promote genetic monogamy and further reinforce pair living by providing benefits for both pair mates. 


\subsection{Open questions and future research directions}

While this study sheds some light on the mechanisms of monogamy maintenance in titis, it leaves many questions unanswered. Some of my findings are based on unquantified or anecdotal observations, and although these findings cannot be used to make definite conclusions, they point the topics that deserve more attention in future studies.

First, as our sample size was only 18 offspring and the maximum number of sampled offspring generations was five, genetic monogamy (or very low level of EPP) in coppery titis will have to be confirmed on a bigger, more long-term dataset. The extent of genetic monogamy in titis also has to be examined in populations with different densities and from different habitats:

- Will coppery titis still have low EPP rates in fragmented or isolated populations where the densities are high and/or opportunities for dispersal are limited?

- Given that titi species inhabit a wide range of different habitats, will the species living in more seasonal habitats and having higher breeding synchrony have higher or lower EPP rates?

In case some EPP are found, it will be possible to test if a proportion of EPP in a pair depends on some characteristics of one or both pair mates:

- Are EPP more common in pairs where the mates are more related, as shown in Alpine marmots (Cohas et al. 2006)?

- Are EPP more common in pairs with higher MHC-similarity between mates, as shown in fat-tailed dwarf lemurs (Schwensow et al. 2008)?

- Do EPP rates increase with male and/or female age, as shown in Eurasian beavers (suggested to be a result of either mate choice or decreased mate guarding by older males) (Nimje et al. 2019)?

- Does male success in gaining EPC depend on his age, as shown in many studies on birds (e.g., Bowers et al., 2015; meta-analysis: Cleasby and Nakagawa, 2012)?

- Are EPP less common in pairs with stronger pair bonds that enable more effective mate guarding?

The absence of relatedness- and heterozygosity-based mate choice in our study population, of course, does not mean that mate choice does not occur in titis at any level. As in other species, titis might choose their mates based on other factors, e.g.:

- Is mate choice in titis based on variation in MHC loci, as shown, for example, in fattailed dwarf lemurs (Schwensow et al. 2008)?

- Is mate choice based on physical condition, such as body mass or size? 
- Is mate choice based on the size or quality of the territory held by a potential mate? To better understand titis' social system, it is crucial to know more about dispersal patterns and floaters. Our study individuals were not fitted with radio-collars and thus dispersing animals could not be tracked. The animals were not marked either, and while we could individually identify study animals when they were near us, we would probably not be able to distinguish a floater from a member of a neighboring group when seeing a solitary animal from the distance. Fitting pre-dispersal age animals with radio-collars and marking them with, e.g., bead collars, in combination with having long-term demographic data, will help to address the following questions:

- How far do animals disperse and what are their movement patterns, i.e., do they settle on the first vacant territory or do they make forays to investigate the available options first?

- Is dispersal delayed in higher-density populations as a result of limited dispersal opportunities?

- Conversely, do animals disperse earlier in higher-density populations because of the increased local competition, meaning that individuals have better perspectives if they move into lower density areas where competition is reduced (Matthysen 2005)?

- Is dispersal delayed in habitats of lower quality or productivity or in more seasonal habitats as a result of limited reproductive opportunities?

- Do floaters evict resident animals as they do in Azara's owl monkeys (FernandezDuque \& Huck 2013)?

Additionally, floater numbers may be assessed by attracting the animals via playback calls and/or using passive acoustic monitoring (Kalan et al. 2015).

Many questions about mate guarding and territorial behaviors could not be addressed in this study because intergroup encounters were infrequent and the reproductive status of females could not be assessed precisely. If long-term observations and data from hormone analyses using fecal samples become available, the following questions can be addressed:

- Do proximity patterns within pairs change during female fertility periods?

- Do space use patterns change during female fertility periods, e.g., do males prevent females from approaching the home range borders or do females approach the borders more to advertise their fertile status to other males?

- Do males participate in the intergroups encounters more often or more actively when their pair mates are fertile or cycling, as shown in white-faced sakis, $P$. pithecia (Thompson et al. 2012)? 
- Do males participate in the intergroups encounters more often or more actively when the females of the neighboring groups are fertile or cycling, as suggested by the interest that males showed in stranger females in captive studies on titis (Anzenberger 1988; Mason 1975; Fernandez-Duque et al. 1997)?

- Do females participate in the intergroups encounters more often or more actively when they are fertile or cycling, as shown in Javan gibbons, H. moloch (Yi et al. 2020)? - Is mate guarding and/or territorial behaviors influenced by pair bond tenure, as suggested by captive studies on P. cupreus where pair mates reacted differently to the simulated intruders depending on the pair bond duration (Rothwell et al. 2020; Fernandez-Duque et al. 2000)?

Finally, territorial behaviors are worth investigating with regard to the population density and habitat characteristics:

- Will intergroup encounters be more frequent and/or intense at higher population densities, as shown, e.g., in North American red squirrels, Tamiasciurus hudsonicus (Dantzer et al. 2012)?

- Alternatively, will intergroup encounters be more frequent and/or intense at lower population density as a result of increased dispersal and thus increased intruder pressure at lower population densities, as shown in Eurasian beavers (Mayer et al. 2020)?

- Will intergroup encounters be more frequent in groups inhabiting home ranges of higher quality because the resident animals would have more to defend?

- Alternatively, will the encounters be more frequent in groups inhabiting home ranges of lower quality because the resident animals would have more to lose?

- In either case, will the pair bond become stronger if the intergroup encounters occur more often as the response to the increased threat to the pair integrity?

Many key aspects of titis' social system, such as the relationship between adult sex ratio, intrasexual competition, mating system and reproductive success, can only be understood with the help of long-term data spanning multiple generations and groups of animals. Hopefully, this study, being the "first-generation" cross-sectional research on coppery titis at our field site, can be the starting point for collecting these long-term data. Finally, comparative studies of social systems of "monogamous" Neotropical primates - titis, sakis, and owl monkeys - will have great potential for the research of the evolution of social and genetic monogamy. 


\section{ACKNOWLEDGEMENTS}

First and foremost, I would like to thank my supervisor, Eckhard W. Heymann, for being the best supervisor I could wish for. I am very grateful for your constant support and for always finding time for me despite having multiple other commitments. I really enjoyed our discussions, they made me think of so many interesting research questions beyond the immediate topic of my project. Thank you for encouraging curiosity and for being a generalist rather than a specialist in science, for always listening to me and for giving me the feeling that my opinion matters. That gave me much confidence as a researcher. Whenever I felt in doubt or lost, I invariably felt better after having a meeting with you. Finally, thank you for taking me as a PhD student and for giving me an amazing opportunity to work in the Peruvian Amazon. I wish everyone was as lucky with their supervisors as I was.

I cannot thank enough the field assistants I worked with in Peru. Camilo Flores Amasifuén and Migdonio Huanuiri Arirama did not only become my dear friends but were also the best guides I could wish for. If it wasn't for them, I would miss half of the exciting animal encounters, and, without doubt, would never learn to individually recognize the monkeys, collect samples or even find my way back to the camp at all. Ney Shahuano Tello, Carlos Caritimari Arirama, Arnaldo Arimuya Flores, Santiago Cariajano Sandi, Aladino Hidalgo Souza, thank you for all your jokes and your great patience in habituating the groups that did not want to be habituated. Thank you so much, all of you, for sharing your knowledge about the forest with me and for waking me up at night to show me the animals. I really hope that soon we will be able work together again.

I am very grateful to my second supervisor, Christian Roos, for giving me the opportunity to work at the Genetic Lab prior to starting my $\mathrm{PhD}$ and for supporting me through all the frustrating months of writing grant proposals and awaiting the decisions. Thank you for your trust in me and for always being positive. Christiane Schwarz, surely I would never finish the lab work without you. Thank you for always staying cool and friendly while doing a million tasks simultaneously. I would also like to thank Oliver Schülke for being on my thesis committee and for the great ideas on how to improve the project.

Sarah Walker and Mathieu Maréchal, who joined my project as field assistants: we had an amazing time. I was incredibly lucky to have you, you made that field season so much fun. Sarina Tiel, thank you for all the evenings and all the laughs. Katya Ovsyanikova, Anna Kaznadzei and Zhenya Solovieva, thank you for being there with me and sharing my love for the forest. 
My Göttingen colleagues who also became dear friends, Tatiana Murillo Corales, Clémence Poirotte, Lotte Striewe, all the others: you made my time here so happy. Thank you for all the drinks, all the lakes, all the climbs and all the dog walks.

I would also like to thank Rosa Vasquez Zanetti for the help with Peruvian bureaucracy and sample export, and for welcoming us in Lima.

I am grateful to Leakey Foundation and International Primatological Society for awarding me the grants that made the second field season possible. This project was also funded by the German Primate Center, Deutsche Forschungsgemeinschaft, and Primate Action Fund.

Finally, but not least importantly, I would like to thank my husband, Sergei Moshkovskiy, for all the love. Thank you for encouraging me to go back to academia, although it meant that I would constantly be away. Thank you for your unfailing curiosity, for being interested in basically everything and for your love and respect for the wildlife. And for all the long hours of waiting for the monkeys and watching barbets. 


\section{REFERENCES}

Achenbach GG, Snowdon CT. 2002. Costs of caregiving: weight loss in captive adult male cotton-top tamarins (Saguinus oedipus) following the birth of infants. Int. J. Primatol. 23:179-189.

Adamack AT, Gruber B. 2014. PopGenReport: simplifying basic population genetic analyses in R. Methods Ecol. Evol. 5:384-387. doi: 10.1111/2041-210X.12158.

Alberts SC, Altmann J, Wilson ML. 1996. Mate guarding constrains foraging activity of male baboons. Anim. Behav. 51:1269-1277. doi: 10.1006/anbe.1996.0131.

Alexander A et al. 2016. What influences the worldwide genetic structure of sperm whales (Physeter macrocephalus)? Mol. Ecol. 25:2754-2772. doi: 10.1111/mec.13638.

Altmann J. 1980. Baboon mothers and infants. Harvard University Press: Cambridge, Massachusetts.

Altmann J, Samuels A. 1992. Costs of maternal care: infant-carrying in baboons. Behav. Ecol. Sociobiol. 29:391-398. doi: 10.1007/BF00170168.

Andersson M, Simmons LW. 2006. Sexual selection and mate choice. Trends Ecol. Evol. 21:296-302. doi: 10.1016/j.tree.2006.03.015.

Anzenberger G. 1992. Monogamous social systems and paternity in primates. In: Paternity in primates: genetic tests and theories. Martin, RD, Dixon, AF, \& Wickings, EJ, editors. Karger: Basel pp. 203-224.

Anzenberger G. 1988. The pairbond in the titi monkey (Callicebus moloch): intrinsic versus extrinsic contributions of the pairmates. Folia Primatol. 50:188-203.

Arct A, Drobniak SM, Cichoń M. 2015. Genetic similarity between mates predicts extrapair paternity - a meta-analysis of bird studies. Behav. Ecol. 26:959-968. doi: 10.1093/beheco/arv004.

Arnold KE, Owens IPF. 2002. Extra-pair paternity and egg dumping in birds: life history, parental care and the risk of retaliation. Proc. R. Soc. B Biol. Sci. 269:1263-1269. doi: 10.1098/rspb.2002.2013.

Baayen RH. 2008. Analyzing linguistic data. Cambridge University Press: Cambridge.

Babb PL, Fernandez-Duque E, Schurr TG. 2015. Oxytocin receptor gene sequences in owl monkeys and other primates show remarkable interspecific regulatory and protein coding variation. Mol. Phylogenet. Evol. 91:160-177. doi: 10.1016/j.ympev.2015.05.006.

Barbian HJ et al. 2018. CHIIMP: an automated high-throughput microsatellite genotyping platform reveals greater allelic diversity in wild chimpanzees. Ecol. Evol. 16:7946-7963. doi: 10.1002/ece3.4302.

Barclay RMR. 1989. The effect of reproductive condition on the foraging behavior of female hoary bats, Lasiurus cinereus. Behav. Ecol. Sociobiol. 24:31-37. doi: 10.1007/BF00300115.

Barelli C et al. 2013. Extra-pair paternity confirmed in wild white-handed gibbons. Am. J. Primatol. 75:1185-1195. doi: 10.1002/ajp.22180.

Barr DJ, Levy R, Scheepers C, Tily HJ. 2013. Random effects structure for confirmatory hypothesis testing: keep it maximal. J. Mem. Lang. 68:255-278. doi: 10.1016/j.jml.2012.11.001.

Barrett L, Halliday JO, Henzi SP. 2006. The ecology of motherhood: the structuring of lactation costs by chacma baboons. J. Anim. Ecol. 75:875-886. doi: 10.1111/j.1365-2656.2006.01105.x.

Bateman AJ. 1948. Intra-sexual selection in Drosophila. Heredity. 2:349-368. doi: 10.1038/hdy.1948.21.

Van Belle S, Fernandez-Duque E, Di Fiore A. 2016. Demography and life history of wild red titi monkeys (Callicebus discolor) and equatorial sakis (Pithecia aequatorialis) in Amazonian Ecuador: a 12-year study. Am. J. Primatol. 78:204-215. doi: 10.1002/ajp.22493.

Van Belle S, Martins A, Fernandez-Duque E, Di Fiore A. 2016. Patterns of paternity in wild socially monogamous titis (Callicebus discolor) and sakis (Pithecia aequatorialis) at the Tiputini Biodiversity Station, Ecuador (conference abstract). In: International Primatological Society and American Society of Primatologists. Chicago, IL.

Bezerra BM, Barnett AA, Souto A, Jones G. 2009. Predation by the tayra on the common marmoset and the pale-throated three-toed sloth. J. Ethol. 27:91-96. doi: 10.1007/s10164-008-0090-3.

Bicca-Marques JC, Garber PA, Azevedo-Lopes MAO. 2002. Evidence of three resident adult male group members in a species of monogamous primate, the red titi monkey (Callicebus cupreus). Mammalia. 66:138-142.

Bicca-Marques JC, Heymann EW. 2013. Ecology and behavior of titi monkeys (genus Callicebus). In: Evolutionary biology and conservation of titis, sakis and uacaris. Barnett, A, Veiga, LM, Ferrari, SF, \& Norconk, MA, editors. Cambridge University Press: New York pp. 196-207. 
Birkhead TR. 1995. Sperm competition: evolutionary causes and consequences. Reprod. Fertil. Dev. 7:755-775. doi: 10.1071/RD9950755.

Birkhead TR, Biggins JD. 1987. Reproductive synchrony and extra-pair copulation in birds. Ethology. 74:320-334. doi: 10.1111/j.1439-0310.1987.tb00942.x.

Bjarnason A, Soligo C, Elton S. 2017. Phylogeny, phylogenetic inference, and cranial evolution in pitheciids and Aotus. Am. J. Primatol. 79:1-11. doi: 10.1002/ajp.22621.

Boesch C, Boesch-Achermann H. 2000. The chimpanzees of the Taï Forest: behavioural ecology and evolution. Oxford University Press.: Oxford.

Boinski S. 1988. Sex differences in the foraging behavior of squirrel monkeys in a seasonal habitat. Behav. Ecol. Sociobiol. 23:177-186. doi: 10.1007/BF00300352.

Bonadonna G et al. 2019. Evidence of genetic monogamy in the lemur Indri (Indri indri). Am. J. Primatol. 81:e22993. doi: 10.1002/ajp.22993.

Bossuyt F. 2002. Natal dispersal of titi monkeys (Callicebus moloch) at Cocha Cashu, Manu National Park. Am. J. Phys. Anthropol. 34.

Boubli JP et al. 2019. On a new species of titi monkey (Primates: Plecturocebus Byrne et al., 2016), from Alta Floresta, southern Amazon, Brazil. Mol. Phylogenet. Evol. 132:117-137. doi: 10.1016/j.ympev.2018.11.012.

Bowers EK et al. 2015. Increased extra-pair paternity in broods of aging males and enhanced recruitment of extra-pair young in a migratory bird. Evolution. 69:2533-2541. doi: 10.1111/evo.12746.

Bowler DE, Benton TG. 2005. Causes and consequences of animal dispersal strategies: relating individual behaviour to spatial dynamics. Biol. Rev. Camb. Philos. Soc. 80:205-225. doi:

$10.1017 /$ S1464793104006645.

Brockelman WY, Reichard U, Treesucon U, Raemaekers JJ. 1998. Dispersal, pair formation and social structure in gibbons (Hylobates lar). Behav. Ecol. Sociobiol. 42:329-339. doi: 10.1007/s002650050445.

Brooker MG, Rowley I, Adams M, Baverstock PR. 1990. Promiscuity: an inbreeding in a socially monogamous species? Behav. Ecol. Sociobiol. 26:191-199.

Brooks ME et al. 2017. glmmTMB balances speed and flexibility among packages for zero-inflated generalized linear mixed modeling. doi: 10.3929/ethz-b-000240890.

Brotherton PNM, Komers PE. 2003. Mate guarding and the evolution of social monogamy in mammals. In: Mating strategies and partnerships in birds, humans and other mammals. Reichard, UH \& Boesch, C, editors. Cambridge University Press: Cambridge, UK pp. 42-58.

Brotherton PNM, Pemberton JM, Komers PE, Malarky G. 1997. Genetic and behavioural evidence of monogamy in a mammal, Kirk's dik-dik (Madoqua kirkii). Proc. R. Soc. London B Biol. Sci. 264:675-681. doi: https://doi.org/10.1098/rspb.1997.0096.

Brouwer L et al. 2017. Multiple hypotheses explain variation in extra-pair paternity at different levels in a single bird family. doi: 10.1111/mec.14385.

Brouwer L, Griffith SC. 2019. Extra-pair paternity in birds. Mol. Ecol. 28:4864-4882. doi: 10.1111/mec.15259.

Brown GR. 2001. Using proximity measures to describe mother-infant relationships. Folia Primatol. 72:80-84.

Brown JL. 1997. A theory of mate choice based on heterozygosity. Behav. Ecol. 8:60-65. doi: 10.1093/beheco/8.1.60.

Burke T, Bruford MW. 1987. DNA fingerprinting in birds. Nature. 327:149-152. doi: 10.1038/327149a0.

Butte NF, King JC. 2005. Energy requirements during pregnancy and lactation. Public Health Nutr. 8:10101027. doi: 10.1079/phn2005793.

Buttery PJ. 1979. Amino acids and other nitrogenous compounds. In: Comparative Animal Nutrition. Rechcigl, M, editor. Karger: Basel pp. 34-79.

Byrne $\mathrm{H}$ et al. 2016. Phylogenetic relationships of the New World titi monkeys (Callicebus): first appraisal of taxonomy based on molecular evidence. Front. Zool. 13:10. doi: 10.1186/s12983-016-0142-4.

Câmara Gusmão A et al. 2019. A new species of titi monkey, Plecturocebus Byrne et al., 2016 (Primates, Pitheciidae), from Southwestern Amazonia, Brazil. Primate Conserv. 22:21-35.

Cäsar C, Byrne R, Young RJ, Zuberbühler K. 2012. The alarm call system of wild black-fronted titi monkeys, Callicebus nigrifrons. Behav. Ecol. Sociobiol. 66:653-667. doi: 10.1007/s00265-011-1313-0. 
Cäsar C, Young RJ. 2008. A case of adoption in a wild group of black-fronted titi monkeys (Callicebus nigrifrons). Primates. 49:146-148. doi: 10.1007/s10329-007-0066-x.

Cäsar C, Zuberbühler K. 2012. Referential alarm calling behaviour in New World primates. Curr. Zool. 58:680-697. doi: 10.1093/czoolo/58.5.680.

Caselli CB, Mennill DJ, Bicca-Marques JC, Setz EZF. 2014. Vocal behavior of black-fronted titi monkeys (Callicebus nigrifrons): acoustic properties and behavioral contexts of loud calls. Am. J. Primatol. 76:788800. doi: 10.1002/ajp.22270.

Caselli CB, Mennill DJ, Gestich CC, Setz EZF, Bicca-Marques JC. 2015. Playback responses of socially monogamous black-fronted titi monkeys to simulated solitary and paired intruders. Am. J. Primatol. 77:1135-1142. doi: 10.1002/ajp.22447.

Van de Casteele T, Galbusera P, Matthysen E. 2001. A comparison of microsatellite-based pairwise relatedness estimators. Mol. Ecol. 10:1539-1549.

Cisneros-Heredia DF, León-Reyes A, Seger S. 2005. Boa constrictor predation on a titi monkey, Callicebus discolor. Neotrop. Primates. 13:11-12. doi: 10.1896/1413-4705.13.3.11.

Cleasby IR, Nakagawa S. 2012. The influence of male age on within-pair and extra-pair paternity in passerines. Ibis. 154:318-324.

Clutton-Brock TH et al. 1998. Costs of cooperative behaviour in suricates (Suricata suricatta). Proc. R. Soc. B Biol. Sci. 265:185-190. doi: 10.1098/rspb.1998.0281.

Clutton-Brock TH. 1989. Mammalian mating systems. Proc. R. Soc. London B Biol. Sci. 236:339-372.

Clutton-Brock TH. 2007. Sexual selection in males and females. Science. 318:1882-1885. doi:

10.1126/science.1133311.

Clutton-Brock TH, Albon SD, Guinness FE. 1989. Fitness costs of gestation and lactation in wild mammals. Nature. 337:260-262. doi: 10.1038/337260a0.

Clutton-Brock TH, Iason GR, Albon SD, Guinness FE. 1982. Effects of lactation on feeding behaviour and habitat use in wild red deer hinds. J. Zool. 198:227-236. doi: 10.1111/j.1469-7998.1982.tb02072.x.

Clutton-Brock TH, Parker GA. 1992. Potential reproductive rates and the operation of sexual selection. Q. Rev. Biol. 67:437-456.

Cohas A et al. 2008. The genetic similarity between pair members influences the frequency of extrapair paternity in alpine marmots. Anim. Behav. 76:87-95. doi: 10.1016/j.anbehav.2008.01.012.

Cohas A, Allainé D. 2009. Social structure influences extra-pair paternity in socially monogamous mammals. Biol. Lett. 5:313-316. doi: 10.1098/rsbl.2008.0760.

Cohas A, Yoccoz NG, Da Silva A, Goossens B, Allainé D. 2006. Extra-pair paternity in the monogamous alpine marmot (Marmota marmota): the roles of social setting and female mate choice. Behav. Ecol. Sociobiol. 59:597-605. doi: 10.1007/s00265-005-0086-8.

Coltman D, Pilkington J, Smith J, Pemberton J. 1999. Parasite-mediated selection against inbred soay sheep in a free-living island population. Evolution (N. Y). 53:1259-1267.

Corley M, Valeggia C, Fernandez-Duque E. 2017. Hormonal correlates of development and natal dispersal in wild female owl monkeys (Aotus azarae) of Argentina. Horm. Behav. 96:42-51. doi: 10.1016/j.yhbeh.2017.08.005.

Cornwallis CK, West SA, Davis KE, Griffin AS. 2010. Promiscuity and the evolutionary transition to complex societies. Nature. 466:969-972. doi: 10.1038/nature09335.

Costa DP, Boeuf BJ Le, Huntley AC, Ortiz CL. 1986. The energetics of lactation in the Northern elephant seal, Mirounga angustirostris. J. Zool. 209:21-33. doi: 10.1111/j.1469-7998.1986.tb03563.x.

Coulon A. 2010. Genhet: an easy-to-use R function to estimate individual heterozygosity. Mol. Ecol. Resour. 10:167-169. doi: 10.1111/j.1755-0998.2009.02731.x.

Crook JH, Gartlan JS. 1966. Evolution of primate societies. Nature. 210:1200-1203. doi:

10.1038/2101200a0.

Cubicciotti DD, Mason WA. 1978. Comparative studies of social behavior in Callicebus and Saimiri: heterosexual jealousy behavior. Behav. Ecol. Sociobiol. 3:311-322. doi: 10.1007/BF00296316.

Curio E. 1978. The adaptive significance of avian mobbing: I. Teleonomic hypotheses and predictions. Z. Tierpsychol. 48:175-183. doi: 10.1111/j.1439-0310.1978.tb00254.x.

Dacier A, De Luna AG, Fernandez-Duque E, Di Fiore A. 2011. Estimating population density of Amazonian titi monkeys (Callicebus discolor) via playback point counts. Biotropica. 43:135-140. doi: 10.1111/j.1744- 


\subsubsection{9.x.}

Dantzer B, Boutin S, Humphries MM, McAdam AG. 2012. Behavioral responses of territorial red squirrels to natural and experimental variation in population density. Behav. Ecol. Sociobiol. 66:865-878. doi: 10.1007/s00265-012-1335-2.

Defler TR. 2004. Primates of Colombia. Conservation International: Bogotá.

Dias PAD, Rangel-Negrín A, Canales-Espinosa D. 2011. Effects of lactation on the time-budgets and foraging patterns of female black howlers (Alouatta pigra). Am. J. Phys. Anthropol. 145:137-146. doi: 10.1002/ajpa.21481.

Dietz JM. 2011. Ecology and social organization of the maned wolf (Chrysocyon brachyurus). Smithson. Contrib. to Zool. 1-51. doi: 10.5479/si.00810282.392.

Dixon A, Ross D, O'Malley SLC, Burke T. 1994. Paternal investment inversely related to degree of extrapair paternity in the reed bunting. Nature. 371:698-700. doi: 10.1038/371698a0.

Dobson AJ, Barnett AG, Barnett AG. 2008. An introduction to generalized linear models. Chapman and Hall/CRC: New York doi: 10.1201/9781584889519.

Dobson SF. 1982. Competition for mates and predominant juvenile male dispersal in mammals. Anim. Behav. 30:1183-1192. doi: 10.1016/S0003-3472(82)80209-1.

Dolotovskaya S, Flores Amasifuen C, Haas CE, Nummert F, Heymann EW. 2019. Active anti-predator behaviour of red titi monkeys (Plecturocebus cupreus). Primate Biol. 6:59-64. doi: 10.5194/pb-6-59-2019.

Dolotovskaya S, Heymann EW. 2020. Do less or eat more: strategies to cope with costs of parental care in a pair-living monkey. Anim. Behav. 163:163-173. doi: 10.1016/j.anbehav.2020.03.012.

Dolotovskaya S, Walker S, Heymann EW. 2019. What makes a pair bond in a Neotropical primate: female and male contributions. R. Soc. Open Sci. 7:191489. doi: https://doi.org/10.1098/rsos.191489.

Doolan SP, Macdonald DW. 1996. Dispersal and extra-territorial prospecting by slender-tailed meerkats (Suricata suricatta) in the south-western Kalahari. J. Zool. 240:59-73. doi: 10.1111/j.14697998.1996.tb05486.x.

Double M, Cockburn A. 2000. Pre-dawn infidelity: females control extra-pair mating in superb fairy-wrens. Proc. R. Soc. B Biol. Sci. 267:465-470. doi: 10.1098/rspb.2000.1023.

Dunbar RIM, Dunbar P. 1988. Maternal time budgets of gelada baboons. Anim. Behav. 36:970-980. doi: 10.1016/S0003-3472(88)80055-1.

Dunbar RIM, Sharman M. 1984. Is social grooming altruistic? Z. Tierpsychol. 64:163-173. doi: 10.1111/j.1439-0310.1984.tb00357.x.

Dutour M, Lena JP, Lengagne T. 2016. Mobbing behaviour varies according to predator dangerousness and occurrence. Anim. Behav. 119:119-124. doi: 10.1016/j.anbehav.2016.06.024.

Eens M, Pinxten R. 1995. Inter-sexual conflicts over copulations in the European starling: evidence for the female mate-guarding hypothesis. Behav Ecol Sociobiol. 36:71-81.

Eggert AK, Sakaluk SK. 1995. Female-coerced monogamy in burying beetles. Behav. Ecol. Sociobiol. 37:147-153. doi: 10.1007/BF00176711.

Ekman J, Bylin A, Tegelström H. 1999. Increased lifetime reproductive success for Siberian jay (Perisoreus infaustus) males with delayed dispersal. Proc. R. Soc. B Biol. Sci. 266:911-915. doi:

10.1098/rspb.1999.0723.

Emlen ST. 1982. The evolution of helping. I. An ecological constraints model. Am. Nat. 119:29-39. doi: $10.1086 / 283888$.

Emlen ST, Oring LW. 1977. Ecology, sexual selection, and the evolution of mating systems. Science. 197:215-223.

Fashing PJ. 2001. Male and female strategies during intergroup encounters in guerezas (Colobus guereza): evidence for resource defense mediated through males and a comparison with other primates. Behav. Ecol. Sociobiol. 50:219-230. doi: 10.1007/s002650100358.

Favre L, Balloux F, Goudet J, Perrin N. 1997. Female-biased dispersal in the monogamous mammal Crocidura russula: evidence from field data and microsatellite patterns. Proc. R. Soc. B Biol. Sci. 264:127132. doi: 10.1098/rspb.1997.0019.

Fedigan LM. 1990. Vertebrate predation in Cebus capucinus: meat eating in a Neotropical monkey. Folia Primatol. 54:196-205. doi: 10.1159/000156444.

Felton A, Felton AM, Wallace RB, Gómez H. 2006. Identification, behavioral observations, and notes on the 
distribution of the titi monkeys Callicebus modestus Lönnberg, 1939 and Callicebus olallae, Lönnberg 1939. Primate Conserv. 20:41-46. doi: 10.1896/0898-6207.20.1.41.

Fernandez-Duque E. 2009. Natal dispersal in monogamous owl monkeys (Aotus azarai) of the Argentinean Chaco. Behaviour. 146:583-606. doi: 10.1163/156853908X397925.

Fernandez-Duque E, Fiore A Di. 2020. The evolution of pair-living, sexual monogamy, and cooperative infant care: insights from research on wild owl monkeys, titi monkeys, sakis, and tamarins. Yearb. Phys. Anthropol. 1-86. doi: 10.1111/j.1365-2958.2003.03935.x.

Fernandez-Duque E, Fiore A Di, de Luna AG. 2013. Pair-mate relationships and parenting in equatorial saki monkeys (Pithecia aequatorialis) and red titi monkeys (Callicebus discolor) of Ecuador. In:

Evolutionary biology and conservation of titis, sakis and uacaris. Veiga, LM, Barnett, AA, Ferrari, SF, \& Norconk, MA, editors. Cambridge University Press: New York pp. 295-302.

Fernandez-Duque E, Huck M. 2013. Till death (or an intruder) do us part: intrasexual-competition in a monogamous primate. PLoS One. 8:e53724. doi: 10.1371/journal.pone.0053724.

Fernandez-Duque E, Mason WA, Mendoza SP. 1997. Effects of duration of separation on responses to mates and strangers in the monogamous titi monkey (Callicebus moloch). Am. J. Primatol. 43:225-237. doi: 10.1002/(SICI)1098-2345(1997)43:3<225::AID-AJP3>3.0.C0;2-Z.

Fernandez-Duque E, Valeggia CR, Mason WA. 2000. Effects of pair-bond and social context on male-female interactions in captive titi monkeys (Callicebus moloch, Primates: Cebidae). Ethology. 106:1067-1082. doi: 10.1046/j.1439-0310.2000.00629.x.

Ferrari SF. 2009. Predation risk and antipredator strategies. In: South American primates. Springer: New York pp. 251-277. doi: 10.1007/978-0-387-78705-3_10.

Ferrari SF, Ferrari MAL. 1990. Predator avoidance behaviour in the buffy-headed marmoset, Callithrix flaviceps. Primates. 31:323-338. doi: 10.1007/BF02381104.

Fietz J et al. 2000. High rates of extra-pair young in the pair-living fat-tailed dwarf lemur, Cheirogaleus medius. Behav. Ecol. Sociobiol. 49:8-17. doi: 10.1007/s002650000269.

Di Fiore A. 2005. A rapid genetic method for sex assignment in non-human primates. Conserv. Genet. 6:1053-1058. doi: 10.1007/s10592-005-9086-5.

Di Fiore A, Fernandez-Duque E, Hurst D. 2007. Adult male replacement in socially monogamous equatorial saki monkeys (Pithecia aequatorialis). Folia Primatol. 78:88-98. doi: 10.1159/000097059.

Di Fiore A, Rendall D. 1994. Evolution of social organization: a reappraisal for primates by using phylogenetic methods. Proc. Natl. Acad. Sci. U. S. A. 91:9941-9945. doi: 10.1073/pnas.91.21.9941.

Foerster K, Delhey K, Johnsen A, Lifjeld JT, Kempenaers B. 2003. Females increase offspring heterozygosity and fitness through extra-pair matings. Nature. 425:714-717. doi: 10.1038/nature01969.

Foerster K, Valcu M, Johnsen A, Kempenaers B. 2006. A spatial genetic structure and effects of relatedness on mate choice in a wild bird population. Mol. Ecol. 15:4555-4567. doi: 10.1111/j.1365-

294X.2006.03091.x.

Forstmeier W, Schielzeth H. 2011. Cryptic multiple hypotheses testing in linear models: overestimated effect sizes and the winner's curse. Behav. Ecol. Sociobiol. 65:47-55. doi: 10.1007/s00265-010-1038-5.

Fox J, Weisberg S. 2011. An R companion to applied regression. Thousand Oaks CA: Sage.

Fragaszy DM, Schwarz S, Shimosaka D. 1982. Longitudinal observations of care and development of infant titi monkeys (Callicebus moloch). Am. J. Primatol. 2:191-200. doi: 10.1002/ajp.1350020207.

Fragaszy DM, Visalberghi E, Fedigan LM. 2004. The complete capuchin: the biology of the genus Cebus. Cambridge University Press: Cambridge.

Frasier TR. 2008. STORM: software for testing hypotheses of relatedness and mating patterns. Mol. Ecol. Resour. 8:1263-1266. doi: 10.1111/j.1755-0998.2008.02358.x.

Fuentes A. 2000. Hylobatid communities: changing views on pair bonding and social organization in hominoids. Am. J. Phys. Anthropol. 31:33-60.

Fuentes A. 2002. Patterns and trends in primate pair bonds. Int. J. Primatol. 23:953-978. doi: 10.1023/A:1019647514080.

Fuentes A. 1999. Re-evaluating primate monogamy. Am. Anthropol. 100:890-907. doi: 10.1525/aa.1998.100.4.890.

Garber PA, Porter LM, Spross J, Di Fiore A. 2016. Tamarins: insights into monogamous and nonmonogamous single female social and breeding systems. Am. J. Primatol. 78:298-314. doi: 10.1002/ajp.22370. 
García-Navas V, Ortego J, Sanz JJ. 2009. Heterozygosity-based assortative mating in blue tits (Cyanistes caeruleus): implications for the evolution of mate choice. Proc. R. Soc. B Biol. Sci. 276:2931-2940. doi: 10.1098/rspb.2009.0417.

Geffen E et al. 2011. Kin encounter rate and inbreeding avoidance in canids. Mol. Ecol. 20:5348-5358. doi: 10.1111/j.1365-294X.2011.05358.x.

Gill LF, van Schaik J, von Bayern AMP, Gahr ML. 2019. Genetic monogamy despite frequent extrapair copulations in "strictly monogamous" wild jackdaws. Behav. Ecol. doi: 10.1093/beheco/arz185.

Goldizen AW. 1987. Facultative polyandry and the role of infant-carrying in wild saddle-back tamarins (Saguinus fuscicollis). Behav Ecol Sociobiol. 20:99-109.

Goslee SC, Urban DL. 2007. The ecodist package for dissimilarity-based analysis of ecological data. J. Stat. Softw. 22:1-19. doi: 10.18637/jss.v022.i07.

Gouy M, Guindon S, Gascuel 0. 2010. SeaView version 4: a multiplatform graphical user interface for sequence alignment and phylogenetic tree building. Mol. Biol. Evol. 27:221-224. doi: 10.1093/molbev/msp259.

Gowaty PA. 1996. Battles of the sexes and origins of monogamy. In: Partnerships in birds: the study of monogamy. Black, JH, editor. Oxford University Press: Oxford pp. 21-52.

Greenwood PJ. 1980. Mating systems, philopatry and dispersal in birds and mammals. Anim. Behav. 28:1140-1162. doi: 10.1016/S0003-3472(80)80103-5.

Griffith SC. 2007. The evolution of infidelity in socially monogamous passerines: neglected components of direct and indirect selection. Am. Nat. 169:274-281. doi: 10.1086/510601.

Griffith SC. 2010. The role of multiple mating and extra-pair paternity in creating and reinforcing boundaries between species in birds. Emu. 110:1-9. doi: 10.1071/MU09057.

Griffith SC, Owens IPF, Thuman KA. 2002. Extra pair paternity in birds: a review of interspecific variation and adaptive function. Mol. Ecol. 11:2195-2212. doi: https://doi.org/10.1046/j.1365-294X.2002.01613.x.

Haghtalab N, Moore N, Heerspink BP, Hyndman DW. 2020. Evaluating spatial patterns in precipitation trends across the Amazon basin driven by land cover and global scale forcings. Theor. Appl. Climatol. 140:411-427. doi: 10.1007/s00704-019-03085-3.

Hall ML. 2004. A review of hypotheses for the functions of avian duetting. Behav. Ecol. Sociobiol. 55:415430. doi: 10.1007/s00265-003-0741-X.

Hansson B et al. 2007. No evidence for inbreeding avoidance in a great reed warbler population. Behav. Ecol. 18:157-164. doi: 10.1093/beheco/arl062.

Harts AMF, Booksmythe I, Jennions MD. 2016. Mate guarding and frequent copulation in birds: a metaanalysis of their relationship to paternity and male phenotype. Evolution (N. Y). 70:2789-2808. doi: 10.1111/evo.13081.

Hassel-Finnegan Heather M et al. 2008. How reliable are density estimates for diurnal primates? Int. J. Primatol. 29:1175-1187. doi: 10.1007/s10764-008-9301-6.

Hennessy CA, Dubach J, Gehrt SD. 2012. Long-term pair bonding and genetic evidence for monogamy among urban coyotes (Canis latrans). J. Mammal. 93:732-742. doi: 10.1644/11-MAMM-A-184.1.

Hershkovitz P. 1990. Titis, New World monkeys of the genus Callicebus (Cebidae, Platyrrhini): a preliminary taxonomic review. Field Museum of Natural History: Chicago.

Heymann EW et al. 2019. Small Neotropical primates promote the natural regeneration of anthropogenically disturbed areas. Sci. Rep. 9:1-9. doi: 10.1038/s41598-019-46683-x.

Heymann EW. 2000. The number of adult males in callitrichine groups and its implications for callitrichine social evolution. In: Primate males: causes and consequences of variation in group composition. Kappeler, PM, editor. Cambridge University Press: Cambridge pp. 64-71.

Heymann EW, Pérez Yamacita JG, Müller B. 2012. Morphometric data from a wild female titi monkey, Callicebus cupreus. Neotrop. Primates. 19:42-43.

Hilgartner R, Fichtel C, Kappeler PM, Zinner D. 2012. Determinants of pair-living in red-tailed sportive lemurs (Lepilemur ruficaudatus). Ethology. 118:466-479. doi: 10.1111/j.1439-0310.2012.02033.x.

Hinde $\mathrm{K}$ et al. 2016. Challenges to the pair bond: neural and hormonal effects of separation and reunion in a monogamous primate. Front. Behav. Neurosci. 10:221. doi: 10.3389/fnbeh.2016.00221.

Hinde RA. 1983. Primate social relationships: an integrated approach. Blackwell: Oxford.

Hinde RA, Atkinson S. 1970. Assessing the roles of social partners in maintaining mutual proximity, as 
exemplified by mother-infant relations in rhesus monkeys. Anim. Behav. 18:169-176. doi: 10.1016/00033472(70)90087-4.

Hoffman JI, Forcada J, Trathan PN, Amos W. 2007. Female fur seals show active choice for males that are heterozygous and unrelated. Nature. 445:912-914. doi: 10.1038/nature05558.

van Hooff J, van Schaik CP. 1992. Cooperation in competition: the ecology of primate bonds. In: Coalitions and alliances in humans and other animals. Harcourt, AH \& DeWaal, F, editors. Oxford University Press: Oxford pp. 397-389.

Hosken DJ, Stockley P, Tregenza T, Wedell N. 2009. Monogamy and the battle of the sexes. Annu. Rev. Entomol. 54:361-378. doi: 10.1146/annurev.ento.54.110807.090608.

Huchard E, Knapp LA, Wang J, Raymond M, Cowlishaw G. 2010. MHC, mate choice and heterozygote advantage in a wild social primate. Mol. Ecol. 19:2545-2561. doi: 10.1111/j.1365-294X.2010.04644.x.

Huck M, Fernandez-Duque E. 2012a. Children of divorce: effects of adult replacements on previous offspring in Argentinean owl monkeys. Behav. Ecol. Sociobiol. 66:505-517. doi: 10.1007/s00265-0111297-9.

Huck M, Fernandez-Duque E. 2017. The floater's dilemma: use of space by wild solitary Azara's owl monkeys, Aotus azarae, in relation to group ranges. Anim. Behav. 127:33-41. doi: 10.1016/j.anbehav.2017.02.025.

Huck M, Fernandez-Duque E. 2012b. When dads help: male behavioral care during primate infant development. In: Building babies: primate development in proximate and ultimate perspective. Clancy, KBH, Hinde, K, \& Rutherford, JN, editors. Springer: New York, NY pp. 361-385. doi: 10.1007/978-1-46144060-4_16.

Huck M, Fernandez-Duque E, Babb PL, Schurr TG. 2014. Correlates of genetic monogamy in socially monogamous mammals: insights from Azara's owl monkeys. Proc. R. Soc. B Biol. Sci. 281:1-8. doi: 10.1098/rspb.2014.0195.

Huck M, Di Fiore A, Fernandez-Duque E. 2020. Of apples and oranges? The evolution of 'monogamy' in non-human primates. Front. Ecol. Evol. 7:472. doi: 10.3389/FEV0.2019.00472.

Huck M, Löttker P, Heymann EW. 2004. The many faces of helping: possible costs and benefits of infant carrying and food transfer in wild moustached tamarins (Saguinus mystax). Behaviour. 141:915-934. doi: $10.1163 / 1568539042265635$.

Hughes WOH, Oldroyd BP, Beekman M, Ratnieks FLW. 2008. Ancestral monogamy shows kin selection is key to the evolution of eusociality. Science. 320:1213-1216. doi: 10.1126/science.1156108.

Isbell LA. 1994. Predation on primates: ecological patterns and evolutionary consequences. Evol. Anthropol. 3:61-71.

Isvaran K, Clutton-Brock TH. 2007. Ecological correlates of extra-group paternity in mammals. Proc. Biol. Sci. 274:219-24. doi: 10.1098/rspb.2006.3723.

Iwamoto T, Mori A, Kawai M, Bekele A. 1996. Anti-predator behavior of gelada baboons. Primates. 37:389-397. doi: 10.1007/BF02381374.

Jaeggi A V, Miles MI, Schradin C, Hayes LD. 2020. Variable social organization is ubiquitous in Artiodactyla and probably evolved from pair-living ancestors. Proc. R. Soc. B Biol. Sci. 287:20200035. doi: http://dx.doi.org/10.1098/rspb.2020.0035.

Jamieson IG, Taylor SS, Tracy LN, Kokko H, Armstrong DP. 2009. Why some species of birds do not avoid inbreeding: insights from New Zealand robins and saddlebacks. Behav. Ecol. 20:575-584. doi: 10.1093/beheco/arp034.

Jantschke B, Welker C, Klaiber-Schuh A. 1995. Notes on breeding of the titi monkey Callicebus cupreus. Folia Primatol. 65:210-213.

Jennions MD, Petrie M. 2000. Why do females mate mulitply? A review of genetic benefits. Biol. Rev. 75:21-64.

Jones OR, Wang J. 2010. COLONY: a program for parentage and sibship inference from multilocus genotype data. Mol. Ecol. Resour. 10:551-555. doi: 10.1111/j.1755-0998.2009.02787.x.

Kalan AK et al. 2015. Towards the automated detection and occupancy estimation of primates using passive acoustic monitoring. Ecol. Indic. 54:217-226. doi: 10.1016/j.ecolind.2015.02.023.

Kalinowski ST, Taper ML, Marshall TC. 2007. Revising how the computer program CERVUS accommodates genotyping error increases success in paternity assignment. Mol. Ecol. 16:1099-1106. doi:

10.1111/j.1365-294X.2007.03089.X. 
Kappeler PM. 2019. A framework for studying social complexity. Behav. Ecol. Sociobiol. 73:13. doi: 10.1007/s00265-018-2601-8.

Kappeler PM. 2014. Lemur behaviour informs the evolution of social monogamy. Trends Ecol. Evol. 29:591-593. doi: 10.1016/j.tree.2014.09.005.

Kappeler PM, Pozzi L. 2019. Evolutionary transitions toward pair living in nonhuman primates as stepping stones toward more complex societies. Sci. Adv. 5:eaay1276. doi: 10.1126/sciadv.aay1276.

Kappeler PM, van Schaik CP. 2002. Evolution of primate social systems. Int. J. Primatol. 23:707-740. doi: 10.1023/A:1015520830318.

Kazem AJN, Widdig A. 2013. Visual phenotype matching: cues to paternity are present in rhesus macaque faces. PLoS One. 8. doi: 10.1371/journal.pone.0055846.

Keller LF, Arcese P. 1998. No evidence for inbreeding avoidance in a natural population of song sparrows (Melospiza melodia). Am. Nat. 152:380-392. doi: 10.1086/286176.

Kempenaers B. 2007. Mate choice and genetic quality: a review of the heterozygosity theory. Adv. Study Behav. 37:189-278. doi: 10.1016/S0065-3454(07)37005-8.

Kempenaers B, Everding S, Bishop C, Boag P, Robertson RJ. 2001. Extra-pair paternity and the reproductive role of male floaters in the tree swallow (Tachycineta bicolor). Behav. Ecol. Sociobiol. 49:251-259. doi: $10.1007 / \mathrm{s} 002650000305$.

Kenyon M, Roos C, Binh VT, Chivers D. 2011. Extrapair paternity in golden-cheeked gibbons (Nomascus gabriellae) in the secondary lowland forest of Cat Tien National Park, Vietnam. Folia Primatol. 82:154164. doi: $10.1159 / 000333143$.

Kinzey WG. 1997. New World primates: ecology, evolution, and behavior. Aldine de Gruyter: New York.

Kinzey WG. 1981. The titi monkeys, genus Callicebus. In: Ecology and behavior of Neotropical primates. Coimbra-Filho, AF \& Mittermeier, RA, editors. Academia Brasileira de Ciências: Rio de Janeiro pp. 241276.

Kinzey WG, Robinson JG. 1983. Intergroup loud calls, range size, and spacing in Callicebus torquatus. Am. J. Phys. Anthropol. 60:539-544.

Kinzey WG, Wright PC. 1982. Grooming behavior in the titi monkey (Callicebus torquatus). Am. J. Primatol. 3:267-275. doi: 10.1002/ajp.1350030124.

Kitchen AM, Gese EM, Waits LP, Karki SM, Schauster ER. 2006. Multiple breeding strategies in the swift fox, Vulpes velox. Anim. Behav. 71:1029-1038. doi: 10.1016/j.anbehav.2005.06.015.

Kleiman DG. 1977. Monogamy in mammals. Q. Rev. Biol. 52:39-69. doi: 10.1086/409721.

Kleiman DG, Malcolm JR. 1981. The evolution of male parental investment in mammals. In: Parental care in mammals. Springer US: Boston, MA pp. 347-387. doi: 10.1007/978-1-4613-3150-6_9.

Klug H. 2018. Why monogamy? A review of potential ultimate drivers. Front. Ecol. Evol. 6:1-6. doi: 10.3389/fevo.2018.00030.

Knogge C, Heymann EW. 1995. Field observation of twinning in the dusky titi monkey, Callicebus cupreus. Folia Primatol. 65:118-120.

Koenig A, Borries C, Chalise MK, Winkler P. 1997. Ecology, nutrition, and timing of reproductive events in an Asian primate, the Hanuman langur (Presbytis entellus). J. Zool. 243:215-235. doi: 10.1111/j.14697998.1997.tb02778.x.

Kokko H, Ekman J. 2002. Delayed dispersal as a route to breeding: territorial inheritance, safe havens, and ecological constraints. Am. Nat. 160:468-484. doi: 10.1086/342074.

Kokko H, Jennions MD. 2008. Parental investment, sexual selection and sex ratios. J. Evol. Biol. 21:919948. doi: 10.1111/j.1420-9101.2008.01540.x.

Komers PE, Brotherton PNM. 1997. Female space use is the best predictor of monogamy in mammals. Proc. R. Soc. B Biol. Sci. 264:1261-1270. doi: 10.1098/rspb.1997.0174.

Kulp J, Heymann EW. 2015. Ranging, activity budget, and diet composition of red titi monkeys (Callicebus cupreus) in primary forest and forest edge. Primates. 56:273-278. doi: 10.1007/s10329-015-0471-5.

Lack D. 1968. Ecological adaptations for breeding in birds. Methuen \& Co: London.

Lambert CT, Sabol AC, Solomon NG. 2018. Genetic monogamy in socially monogamous mammals is primarily predicted by multiple life history factors: a meta-analysis. Front. Ecol. Evol. 6:139. doi: 10.3389/fevo.2018.00139.

Lawrence J. 2007. Understanding the pair bond in brown titi monkeys (Callicebus brunneus): male and 
female reprodcutive interests (PhD thesis). Columbia University, New York.

Leclaire S, Nielsen JF, Sharp SP, Clutton-Brock TH. 2013. Mating strategies in dominant meerkats: evidence for extra-pair paternity in relation to genetic relatedness between pair mates. J. Evol. Biol. 26:1499-1507. doi: $10.1111 /$ jeb.12151.

Lee AG et al. 2011. A novel form of oxytocin in new world monkeys. Biol. Lett. 7:584-587. doi: 10.1098/rsbl.2011.0107.

Lee PC. 1987. Nutrition, fertility and maternal investment in primates. J. Zool. 213:409-422. doi: 10.1111/j.1469-7998.1987.tb03717.x.

Lee PC, Majluf P, Gordon IJ. 1991. Growth, weaning and maternal investment from a comparative perspective. J. Zool. 225:99-114. doi: 10.1111/j.1469-7998.1991.tb03804.x.

Leedale AE et al. 2020. Cost, risk, and avoidance of inbreeding in a cooperatively breeding bird. Proc. Natl. Acad. Sci. 201918726. doi: 10.1073/pnas.1918726117.

Leigh JW, Bryant D. 2015. POPART: Full-feature software for haplotype network construction. Methods Ecol. Evol. 6:1110-1116. doi: 10.1111/2041-210X.12410.

Li CC, Weeks DE, Chakravarti A. 1993. Similarity of DNA fingerprints due to chance and relatedness. Hum. Hered. 43:45-52. doi: 10.1159/000154113.

Lima SL. 1987. Vigilance while feeding and its relation to the risk of predation. J. Theor. Biol. 124:303-316. doi: 10.1016/S0022-5193(87)80118-2.

Lukas D, Clutton-Brock TH. 2013. The evolution of social monogamy in mammals. Science. 341:526-530. doi: $10.1126 /$ science.1238677.

Lukas D, Huchard E. 2014. The evolution of infanticide by males in mammalian societies. Science. 346:841-844. doi: 10.1126/science.1257226.

De Luna AG, Sanmiguel R, Di Fiore A, Fernandez-Duque E. 2010. Predation and predation attempts on red titi monkeys (Callicebus discolor) and equatorial sakis (Pithecia aequatorialis) in Amazonian Ecuador. Folia Primatol. 81:86-95. doi: 10.1159/000314948.

Lynch M, Ritland K. 1999. Estimation of pairwise relatedness with molecular markers. Genetics. 152:1753-1766.

MacManes MD. 2011. Promiscuity in mice is associated with increased vaginal bacterial diversity. Naturwissenschaften. 98:951-960. doi: 10.1007/s00114-011-0848-2.

Manson JH. 1997. Primate consortships: a critical review. Curr. Anthropol. 38:353-374. doi: $10.1086 / 204623$.

Martins AB. 2015. Characterization and evaluation of microsatellite loci suitable for studies on mating system, parentage, and genetic identity in red titi monkeys (Callicebus discolor) and saki monkeys (Pithecia aequatorialis). The University of Texas at Austin.

Mason WA. 1975. Comparative studies of social behavior in Callicebus and Saimiri: strength and specificity of attraction between male-female cagemates. Folia Primatol. 23:113-123. doi: 10.1159/000155664.

Mason WA. 1966. Social organization of the South American monkey, Callicebus moloch: a preliminary report. Tulane Stud Zool. 13:23-28.

Mateo JM. 2017. The ontogeny of kin-recognition mechanisms in Belding's ground squirrels. Physiol. Behav. 173:279-284. doi: 10.1016/j.physbeh.2017.02.024.

Matthysen E. 2005. Density-dependent dispersal in birds and mammals. Ecography. 28:403-416. doi: 10.1111/j.0906-7590.2005.04073.x.

Matuschek H, Kliegl R, Vasishth S, Baayen H, Bates D. 2017. Balancing Type I error and power in linear mixed models. J. Mem. Lang. 94:305-315. doi: 10.1016/J.JML.2017.01.001.

Mayer M, Frank SC, Zedrosser A, Rosell F. 2020. Causes and consequences of inverse density-dependent territorial behaviour and aggression in a monogamous mammal. J. Anim. Ecol. 89:577-588. doi: 10.1111/1365-2656.13100.

Mayer M, Zedrosser A, Rosell F. 2017a. Couch potatoes do better: delayed dispersal and territory size affect the duration of territory occupancy in a monogamous mammal. Ecol. Evol. 7:4347-4356. doi: 10.1002/ece3.2988.

Mayer M, Zedrosser A, Rosell F. 2017b. When to leave: the timing of natal dispersal in a large, monogamous rodent, the Eurasian beaver. Anim. Behav. 123:375-382. doi: 10.1016/j.anbehav.2016.11.020. 
Maynard Smith S. 1977. Parental investment: a prospective analysis. Anim. Behav. 25:1-9.

McClanahan K, Rosell F, Mayer M. 2020. Minding your own business: low pair cohesion in a territorial , monogamous mammal. Anim. Behav. 166:119-128. doi: 10.1016/j.anbehav.2020.05.008.

Mendoza A et al. 2015. Population genetics of the California National Primate Research Center's (CNPRC) captive Callicebus cupreus colony. Primates. 56:37-44. doi: 10.1007/s10329-014-0446-y.

Mendoza SP, Mason WA. 1986. Contrasting responses to intruders and to involuntary separation by monogamous and polygynous New World monkeys. Physiol. Behav. 38:795-801. doi: 10.1016/00319384(86)90045-4.

Menescal LA, Gonçalves EC, Silva A, Ferrari SF, Schneider MPC. 2009. Genetic diversity of red-bellied titis (Callicebus moloch) from Eastern Amazonia based on microsatellite markers. Biochem. Genet. 47:235240. doi: 10.1007/s10528-008-9220-4.

Miller LE. 2002. Introduction to predator sensitive foraging. In: Eat or be eaten: predator sensitive foraging among primates. Miller, LE, editor. Cambridge University Press: Cambridge pp. 1-17.

Murray CM, Lonsdorf E V., Eberly LE, Pusey AE. 2009. Reproductive energetics in free-living female chimpanzees (Pan troglodytes schweinfurthii). Behav. Ecol. 20:1211-1216. doi: 10.1093/beheco/arp114.

Nadjafzadeh MN, Heymann EW. 2008. Prey foraging of red titi monkeys, Callicebus cupreus, in comparison to sympatric tamarins, Saguinus mystax and Saguinus fuscicollis. Am. J. Phys. Anthropol. 135:56-63. doi: 10.1002/ajpa.20704.

Nimje PS et al. 2019. Almost faithful: SNP markers reveal low levels of extra-pair paternity in the Eurasian beavers. PeerJ Prepr. 7:e27866v1. doi: 10.7287/peerj.preprints.27866v1.

Nishida T. 1988. Development of social grooming between mother and offspring in wild chimpanzees. Folia Primatol. 50:109-123. doi: 10.1159/000156335.

Norconk MA. 2020. Historical antecedents and recent innovations in pitheciid (titi, saki, and uakari) feeding ecology. Am. J. Primatol. 1-27. doi: 10.1002/ajp.23177.

Norconk MA. 2006. Long-term study of group dynamics and female reproduction in Venezuelan Pithecia pithecia. Int. J. Primatol. 27:653-674. doi: 10.1007/s10764-006-9030-7.

Norconk MA. 2011. Sakis, uakaris, and titi monkeys: behavioral diversity in a radiation of primate seed predators. In: Primates in perspective. Campbell, CJ, Fuentes, A, MacKinnon, KC, Bearder, SK, \& Stumpf, RM, editors. Oxford University Press: New York, NY pp. 122-139.

Norconk MA, Setz E. 2013. Ecology and behavior of saki monkeys (genus Pithecia). In: Evolutionary biology and conservation of titis, sakis and uacaris. Veiga, L, Barnett, A, Ferrari, S, \& Norconk, MA, editors. Cambridge University Press: New York pp. 262-271.

Nunn CL, Altizer S. 2006. Infectious diseases in primates: behavior, ecology and evolution. Oxford University Press: Oxford.

Oka T, Takenaka 0. 2001. Wild gibbons' parentage tested by non-invasive DNA sampling and PCRamplified polymorphic microsatellites. Primates. 42:67-73. doi: 10.1007/BF02640690.

Oliveira FB De, Molina EC, Marroig G. 2009. Paleogeography of the South Atlantic: a route for primates and rodents into the New World? In: South American primates. Springer: New York pp. 55-68. doi: 10.1007/978-0-387-78705-3.

Van Oosterhout C, Hutchinson WF, Wills DPM, Shipley P. 2004. MICRO-CHECKER: software for identifying and correcting genotyping errors in microsatellite data. Mol. Ecol. Notes. 4:535-538. doi: 10.1111/j.14718286.2004.00684.x.

Opie C, Atkinson QD, Dunbar RIM, Shultz S. 2013. Male infanticide leads to social monogamy in primates. Proc. Natl. Acad. Sci. U. S. A. 110:13328-32. doi: 10.1073/pnas.1307903110.

Orians GH. 1969. On the evolution of mating systems in birds and mammals. Am. Nat. 103:589-603.

Ortego J, Calabuig G, Cordero PJ, Aparicio JM. 2007. Egg production and individual genetic diversity in lesser kestrels. Mol. Ecol. 16:2383-2392. doi: 10.1111/j.1365-294X.2007.03322.x.

Osorio-Beristain M, Drummond H. 2001. Male boobies expel eggs when paternity is in doubt. Behav. Ecol. 12:16-21. doi: 10.1093/oxfordjournals.beheco.a000373.

Ossi K, Kamilar JM. 2006. Environmental and phylogenetic correlates of Eulemur behavior and ecology (Primates: Lemuridae). Behav. Ecol. Sociobiol. 61:53-64. doi: 10.1007/s00265-006-0236-7.

Palacios E, Rodríguez A, Defler TR. 1997. Diet of a group of Callicebus torquatus lugens (Humboldt, 1812) during the annual resource bottleneck in amazonian Colombia. Int. J. Primatol. 18:503-522. doi:

10.1023/A:1026307121583. 
Palombit RA. 2000. Infanticide and male-female relationships. In: Infanticide by males and its implications. Van Schaik, CP \& Janson, CH, editors. Cambridge University Press: Cambridge pp. 239-268.

Palombit RA, Cargile PL, Singh D, Kaur M. 1996. Pair bonds in monogamous apes: a comparison of the siamang Hylobates syndactylus and the white-handed gibbon Hylobates lar. Behaviour. 133:321-356.

Parreira B, Quéméré E, Vanpé C, Carvalho I, Chikhi L. 2020. Genetic consequences of social structure in the golden-crowned sifaka. Heredity. 1-12. doi: 10.1038/s41437-020-0345-5.

Penteriani V, Ferrer M, Delgado MM. 2011. Floater strategies and dynamics in birds, and their importance in conservation biology: towards an understanding of nonbreeders in avian populations. Anim. Conserv. 14:233-241. doi: 10.1111/j.1469-1795.2010.00433.x.

Perelman P et al. 2011. A molecular phylogeny of living primates. PLoS Genet. 7:1-17. doi: 10.1371/journal.pgen.1001342.

Petrie M, Kempenaers B. 1998. Extra-pair paternity in birds: explaining variation between species and populations. Trends Ecol. Evol. 13:52-58. doi: 10.1016/S0169-5347(97)01232-9.

Pew J, Muir PH, Wang J, Frasier TR. 2015. related: an R package for analysing pairwise relatedness from codominant molecular markers. Mol. Ecol. Resour. 15:557-561. doi: 10.1111/1755-0998.12323.

Pfefferle D, Ruiz-Lambides A V., Widdig A. 2013. Female rhesus macaques discriminate unfamiliar paternal sisters in playback experiments: support for acoustic phenotype matching. Proc. R. Soc. B Biol. Sci. 281. doi: 10.1098/rspb.2013.1628.

Podolsky RD. 1990. Effects of mixed-species association on resource use by Saimiri sciureus and Cebus apella. Am. J. Primatol. 21:147-158. doi: 10.1002/ajp.1350210207.

Poiani A, Wilks C. 2000. Sexually transmitted diseases: a possible cost of promiscuity in birds? Auk. 117:1061-1065. doi: 10.1093/AUK/117.4.1061.

Pollock JI. 1979. Female dominance in Indri indri. Folia Primatol. 31:143-164. doi: 10.1159/000155877.

Porter AM. 2016. Variability in the social lives of red titi monkeys (Callicebus discolor) and equatorial saki monkeys (Pithecia aequatorialis), two pair-living primates in Ecuador. University of California, Davis.

Porter AM, Grote MN, Fernandez-Duque E, Di Fiore A, Isbell LA. 2017. Delayed dispersal and immigration in equatorial sakis (Pithecia aequatorialis): factors in the transition from pair- to group-living. Folia Primatol. 88:11-27. doi: 10.1159/000464147.

Presley SJ. 2000. Eira barbara. Mamm. species. 636:1-6.

Price EC. 1992a. Changes in the activity of captive cotton-top tamarins (Saguinus oedipus) over the breeding cycle. Primates. 33:99-106.

Price EC. 1992b. The costs of infant carrying in captive cotton-top tamarins. Am. J. Primatol. 26:23-33. doi: 10.1002/ajp.1350260106.

Price EC, Piedade HM. 2001. Ranging behavior and intraspecific relationships of masked titi monkeys (Callicebus personatus personatus). Am. J. Primatol. 53:87-92. doi: 10.1002/10982345(200102)53:2<87::AID-AJP4>3.0.C0;2-P.

Quinn GP, Keough MJ. 2002. Experimental designs and data analysis for biologists. Cambridge University Press: Cambridge.

R Core Team. 2018. R: A language and environment for statistical computing.

Ramos AG et al. 2014. Habitat structure and colony structure constrain extrapair paternity in a colonial bird. Anim. Behav. 95:121-127. doi: 10.1016/j.anbehav.2014.07.003.

Ribble DO. 1992. Dispersal in a monogamous rodent, Peromyscus californicus. Ecology. 73:859-866.

Ribble D0. 1991. The monogamous mating system of Peromyscus californicus as revealed by DNA fingerprinting. Behav. Ecol. Sociobiol. 29:161-166. doi: 10.1007/BF00166397.

Rioux-Paquette E, Festa-Bianchet M, Coltman DW. 2010. No inbreeding avoidance in an isolated population of bighorn sheep. Anim. Behav. 80:865-871. doi: 10.1016/j.anbehav.2010.08.006.

Robinson JG. 1981. Vocal regulation of inter- and intragroup spacing during boundary encounters in the titi monkey, Callicebus moloch. Primates. 22:161-172. doi: 10.1007/BF02382607.

Robinson JG. 1979. Vocal regulation of use of space by groups of titi monkeys Callicebus moloch. Behav. Ecol. Sociobiol. 5:1-15. doi: 10.1007/BF00302691.

Rodman PS, Bossuyt FJ. 2007. Fathers and stepfathers: familial relations of old and new males within groups of Callicebus brunneus in southeastern Peru [Abstract]. Am J Phys Anthr. 132:201.

van Roosmalen MGM, van Roosmalen T, Mittermeier R a. 2002. A taxonomic review of the titi monkeys, 
genus Callicebus Thomas, 1903, with the description of two new species, Callicebus bernhardi and Callicebus stephennashi, from Brazilian Amazonia. Neotrop. Primates. 10:1-52. doi: 10.1007/s10533-0079087-1.

Rosenberger AL, Marcelo FT. 2013. The misbegotten: long lineages, long branches and the interrelationships of Aotus, Callicebus and the saki-uacaris. In: Evolutionary biology and conservation of titis, sakis and uacaris. Cambridge University Press: Cambridge pp. 13-22.

Rothwell ES, Carp SB, Savidge LE, Mendoza SP, Bales KL. 2020. Relationship tenure differentially influences pair-bond behavior in male and female socially monogamous titi monkeys (Callicebus cupreus). Am. J. Primatol. 1-12. doi: 10.1002/ajp.23181.

Runcie MJ. 2000. Biparental care and obligate monogamy in the rock-haunting possum, Petropseudes dahli, from tropical Australia. Anim. Behav. 59:1001-1008. doi: 10.1006/ANBE.1999.1392.

Sampaio DT, Ferrari SF. 2005. Predation of an infant titi monkey (Callicebus moloch) by a tufted capuchin (Cebus apella). Folia Primatol. 76:113-115. doi: 10.1159/000083617.

Sánchez S, Peláez F, Gil-Bürmann C, Kaumanns W, Sánchez Rodríguez S. 1999. Costs of infant-carrying in the cotton-top tamarin (Saguinus oedipus). Am. J. Primatol. 48:99-111.

Sandell MI, Diemer M. 1999. Intraspecific brood parasitism: a strategy for floating females in the European starling. Anim. Behav. 57:197-202. doi: 10.1006/anbe.1998.0936.

de Santana MM, Souza-Alves JP, Ferrari SF. 2014. Twinning in titis (Callicebus coimbrai): stretching the limits of biparental infant caregiving? Neotrop. Primates. 21:189-191. doi: 10.1896/044.021.0205.

Santema P, Clutton-Brock TH. 2013. Meerkat helpers increase sentinel behaviour and bipedal vigilance in the presence of pups. Anim. Behav. 85:655-661. doi: 10.1016/j.anbehav.2012.12.029.

Savagian A, Fernandez-Duque E. 2017. Do predators and thermoregulation influence choice of sleeping sites and sleeping behavior in Azara's owl monkeys (Aotus azarae azarae) in Northern Argentina? Int. J. Primatol. 38:80-99. doi: 10.1007/s10764-016-9946-5.

Say L, Devillard S, Natoli E, Pontier D. 2002. The mating system of feral cats (Felis catus L.) in a subAntarctic environment. Polar Biol. 25:838-842. doi: 10.1007/s00300-002-0427-2.

Say L, Pontier D, Natoli E. 1999. High variation in multiple paternity of domestic cats (Felis catus L.) in relation to environmental conditions. Proc. R. Soc. B Biol. Sci. 266:2071-2074. doi: 10.1098/rspb.1999.0889.

van Schaik CP. 1983. Why are diurnal primates living in groups? Behaviour. 87:120-144. doi: $10.2307 / 4534297$.

van Schaik CP, Dunbar RIM. 1990. The evolution of monogamy in large primates: a new hypothesis and some crucial tests. Behaviour. 115.

van Schaik CP, Kappeler PM. 2003. The evolution of social monogamy in primates. In: Monogamy: mating strategies and partnerships in birds, humans, and other mammals. Reichard, UH \& Boesch, C, editors. Cambridge University Press: Cambridge pp. 59-80. doi: 10.1017/CB09781139087247.004.

van Schaik CP, van Noordwijk MA. 1989. The special role of male Cebus monkeys in predation avoidance and its effect on group composition. Behav. Ecol. Sociobiol. 24:265-276. doi: 10.1007/BF00290902.

Schielzeth H. 2010. Simple means to improve the interpretability of regression coefficients. Methods Ecol. Evol. 1:103-113. doi: 10.1111/j.2041-210X.2010.00012.x.

Schneider H, Sampaio I. 2015. The systematics and evolution of New World primates - a review. Mol. Phylogenet. Evol. 82:348-357. doi: 10.1016/j.ympev.2013.10.017.

Schülke 0. 2003. To breed or not to breed — food competition and other factors involved in female breeding decisions in the pair-living nocturnal fork-marked lemur (Phaner furcifer). Behav. Ecol. Sociobiol. 55:11-21. doi: 10.1007/s00265-003-0676-2.

Schülke 0, Kappeler PM. 2003. So near and yet so far: territorial pairs but low cohesion between pair partners in a nocturnal lemur, Phaner furcifer. Anim. Behav. 65:331-343. doi: 10.1006/anbe.2003.2018.

Schultz A et al. 2020. Inbreeding and disease avoidance in a free-ranging koala population. Mol. Ecol. 29:2416-2430. doi: 10.1111/mec.15488.

Schwensow N, Fietz J, Dausmann K, Sommer S. 2008. MHC-associated mating strategies and the importance of overall genetic diversity in an obligate pair-living primate. Evol. Ecol. 22:617-636. doi: 10.1007/s10682-007-9186-4.

Serio-Silva JC, Hernandez-Salazar LT, Rico-Gray V. 1999. Nutritional composition of the diet of Alouatta palliata mexicana females in different reproductive states. Zoo Biol. 18:507-513. doi: 
10.1002/(SICI)1098-2361(1999)18:6<507::AID-ZO05>3.0.CO;2-R.

Shahuano Tello N, Huck M, Heymann EW. 2002. Boa constrictor attack and group defense in moustached tamarins (Saguinus mystax). Folia Primatol. 73:146-148.

Sheldon BC. 1994. Male phenotype, fertility, and the pursuit of extra-pair copulations by female birds. Proc. R. Soc. London. Ser. B Biol. Sci. 257:25-30. doi: 10.1098/rspb.1994.0089.

Shultz S, Opie C, Atkinson QD. 2011. Stepwise evolution of stable sociality in primates. Nature. 479:21922. doi: $10.1038 /$ nature 10601 .

Shuster SM, Willen RM, Keane B, Solomon NG. 2019. Alternative mating tactics in socially monogamous prairie voles, Microtus ochrogaster. Front. Ecol. Evol. 7. doi: 10.3389/fevo.2019.00007.

Sikes RS. 1995. Costs of lactation and optimal litter size in northern grasshopper mice (Onychomys leucogaster). J. Mammal. 76:348-357. doi: 10.2307/1382346.

Silvestro D et al. 2019. Early arrival and climatically-linked geographic expansion of New World monkeys from tiny African ancestors. Syst. Biol. 68:78-92. doi: 10.1093/sysbio/syy046.

Sin YW et al. 2015. MHC class II-assortative mate choice in European badgers (Meles meles). Mol. Ecol. 24:3138-3150. doi: 10.1111/mec.13217.

Small MF. 1990. Alloparental behaviour in Barbary macaques, Macaca sylvanus. Anim. Behav. 39:297-306. doi: 10.1016/S0003-3472(05)80874-7.

Smith RJ, Jungers WL. 1997. Body mass in comparative primatology. J. Hum. Evol. 32:523-559. doi: 10.1006/jhev.1996.0122.

Smithson M, Verkuilen J. 2006. A better lemon squeezer? Maximum-likelihood regression with betadistributed dependent variables. Psychol. Methods. 11:54-71. doi: 10.1037/1082-989X.11.1.54.

Smouse PE, Peakall R. 1999. Spatial autocorrelation analysis of individual multiallele and multilocus genetic structure. Heredity. 82:561-573. doi: 10.1038/sj.hdy.6885180.

Smuts BB, Gubernick DJ. 1992. Male-infant relationships in nonhuman primates: paternal investment or mating effort? In: Father-child relations: cultural and biosocial contexts. Hewlett, BS, editor. Routledge: Abingdon pp. 1-30.

Soini P. 1988. The Pygmy Marmoset, genus Cebuella. In: Ecology and Behavior of Neotropical Primates. Mittermeier, RA, Rylands, AB, Coimbra-Filho, AF, \& Da Fonseca, GAB, editors. World Wildlife Fund: Washington pp. 79-129.

Sommer S. 2005. Major histocompatibility complex and mate choice in a monogamous rodent. Behav. Ecol. Sociobiol. 58:181-189. doi: 10.1007/s00265-005-0909-7.

Sommer S, Tichy H. 1999. Major histocompatibility complex (MHC) class II polymorphism and paternity in the monogamous Hypogeomys antimena, the endangered, largest endemic Malagasy rodent. Mol. Ecol. 8:1259-1272. doi: 10.1046/j.1365-294X.1999.00687.x.

Souza-Alves JP, Caselli CB, Gestich CC, Nagy-Reis MB. 2019. Should I store, or should I sync? The breeding strategy of two small Neotropical primates under predictable resource availability. Primates. 60:113-118. doi: 10.1007/s10329-019-00716-1.

Spence-Aizenberg A, Di Fiore A, Fernandez-Duque E. 2016. Social monogamy, male-female relationships, and biparental care in wild titi monkeys (Callicebus discolor). Primates. 57:103-112. doi: 10.1007/s10329-015-0489-8.

Stutchbury BJ, Morton ES. 1995. The effect of breeding synchrony on extra-pair mating systems in songbirds. Behaviour. 132:675-690.

Syrůčková A et al. 2015. Genetic relationships within colonies suggest genetic monogamy in the Eurasian beaver (Castor fiber). Mammal Res. 60:139-147. doi: 10.1007/s13364-015-0219-z.

Szulkin M, Sheldon BC. 2008. Dispersal as a means of inbreeding avoidance in a wild bird population. Proc. R. Soc. B Biol. Sci. 275:703-711. doi: 10.1098/rspb.2007.0989.

Tardif SD. 1994. Relative energetic cost of infant care in small-bodied neotropical primates and its relation to infant-care patterns. Am. J. Primatol. 34:133-143. doi: 10.1002/ajp.1350340205.

Tardif SD. 1997. The bioenergetics of parental behavior and the evolution off alloparental care in marmosets and tamarins. In: Cooperative breeding in mammals. Solomon, NG \& French, JA, editors. Cambridge University Press: Cambridge pp. 11-34.

Tecot SR, Singletary B, Eadie E. 2016. Why 'monogamy’ isn't good enough. Am. J. Primatol. 78:340-354. doi: 10.1002/ajp.22412. 
Terborgh J, Janson CH. 1986. The socioecology of primate groups. Annu. Rev. Ecol. Syst. Vol. 17. 111-136. doi: 10.1146/annurev.es.17.110186.000551.

Terrones Ruiz WI, Vela Diaz DM, Flores Amasifuen C, Heymann EW. 2004. Diurnal birth of a wild red titi monkey, Callicebus cupreus, at the Estacion Biologica Quebrada Blanco. Neotrop. Primates. 12:15-16.

Thierry B, Iwaniuk AN, Pellis SM. 2000. The influence of phylogeny on the social behaviour of macaques (Primates: Cercopithecidae, genus Macaca). Ethology. 106:713-728. doi: 10.1046/j.1439-

0310.2000.00583.x.

Thompson CL, Norconk M, Norconk MA, Whitten PL. 2012. Why fight? Selective forces favoring betweengroup aggression in a variably pair-living primate, the white-faced saki (Pithecia pithecia). Behaviour. 149:795-820. doi: 10.2307/41720587.

Thompson CL, Norconk MA. 2011. Within-group social bonds in white-faced saki monkeys (Pithecia pithecia) display male-female pair preference. Am. J. Primatol. 73:1051-1061. doi: 10.1002/ajp.20972.

Tirado Herrera ER, Heymann EW. 2004. Does mom need more protein? Preliminary observations on differences in diet composition in a pair of red titi monkeys (Callicebus cupreus). Folia Primatol. 75:150153. doi: $10.1159 / 000078304$.

Trivers RL. 1972. Parental investment and sexual selection. In: Sexual selection and the descent of man. Campbell, B, editor. Aldine: Chicago pp. 136-179.

Turjeman SF et al. 2016. Extra-pair paternity in the socially monogamous white stork (Ciconia ciconia) is fairly common and independent of local density. Sci. Rep. 6:1-9. doi: 10.1038/srep27976.

Valeggia CR, Mendoza SP, Fernandez-Duque E, Mason WA, Lasley B. 1999. Reproductive biology of female titi monkeys (Callicebus moloch) in captivity. Am. J. Primatol. 47:183-195. doi: 10.1002/(SICI)10982345(1999)47:3<183::AID-AJP1>3.0.CO;2-J.

Valencia LM, Martins A, Ortiz EM, Di Fiore A. 2019. A RAD-sequencing approach to genome-wide marker discovery, genotyping, and phylogenetic inference in a diverse radiation of primates. doi: 10.1371/journal.pone.0201254.

Vargas-Pinilla P et al. 2015. Evolutionary pattern in the OXT-OXTR system in primates: coevolution and positive selection footprints. Proc. Natl. Acad. Sci. 112:88-93. doi: 10.1073/pnas.1419399112.

Vasey N. 2004. Circadian rhythms in diet and habitat use in red ruffed lemurs (Varecia rubra) and whitefronted brown lemurs (Eulemur fulvus albifrons). Am. J. Phys. Anthropol. 124:353-363. doi:

10.1002/ajpa.10357.

Veiga LM, Barnett AA, Ferrari SF, Norconk MA, eds. 2013. Evolutionary biology and conservation of titis, sakis and uacaris. Cambridge University Press: Cambridge.

Wagner M, Castro F, Stevenson PR. 2009. Habitat characterization and population status of the dusky titi (Callicebus ornatus) in fragmented forests, Meta, Colombia. Neotrop. Primates. 16:18-24. doi: 10.1896/044.016.0104.

Wang J. 2002. An estimator for pairwise relatedness using molecular markers. Genetics. 160:1203-1215.

Wang J. 2011. Coancestry: a program for simulating, estimating and analysing relatedness and inbreeding coefficients. Mol. Ecol. Resour. 11:141-145. doi: 10.1111/j.1755-0998.2010.02885.x.

West HER, Capellini I. 2016. Male care and life history traits in mammals. Nat. Commun. 7. doi: $10.1038 /$ ncomms11854.

Westneat DF. 1990. The ecology and evolution of extra-pair copulations in birds. Curr. Ornithol. 7:331369.

Westneat DF, Sherman PW. 1997. Density and extra-pair fertilizations in birds: a comparative analysis. Behav. Ecol. Sociobiol. 41:205-215. doi: 10.1007/s002650050381.

Westneat DF, Stewart IRK. 2003. Extra-pair paternity in birds: causes, correlates, and conflict. Annu. Rev. Ecol. Evol. Syst. 34:365-396. doi: 10.1146/annurev.ecolsys.34.011802.132439.

Whittingham LA, Dunn PO. 2001. Male parental care and paternity in birds. Curr. Ornithol. 16:257-298. doi: 10.1007/978-1-4615-1211-0_5.

Wittenberger JF, Tilson RL. 1980. The evolution of monogamy: hypotheses and evidence. Annu. Rev. Ecol. Syst. 11:197-232. doi: 10.1146/annurev.es.11.110180.001213.

Wolff J0. 1992. Parents suppress reproduction and stimulate dispersal in opposite-sex juvenile whitefooted mice. Nature. 359:409-410.

Wolff JO, MacDonald DW. 2004. Promiscuous females protect their offspring. Trends Ecol. Evol. 19:127134. doi: 10.1016/j.tree.2003.12.009. 
Wolovich CK, Evans S. 2007. Sociosexual behavior and chemical communication of Aotus nancymaae. Int. J. Primatol. 28:1299-1313. doi: 10.1007/s10764-007-9228-3.

Wolovich CK, Tapanes E, Evans S. 2017. Allogrooming in male-memale pairs of captive owl monkeys (Aotus nancymaae). Folia Primatol. 88:483-496. doi: 10.1159/000485134.

Woodroffe R, Vincent A. 1994. Mother's little helpers: patterns of male care in mammals. Trends Ecol. Evol. 9:294-297. doi: 10.1016/0169-5347(94)90033-7.

Wrangham RW. 1980. An ecological model of female-bonded primate groups. Behaviour. 75:262-300. doi: 10.1163/156853980X00447.

Wright HWY. 2006. Paternal den attendance is the best predictor of offspring survival in the socially monogamous bat-eared fox. Anim. Behav. 71:503-510. doi: 10.1016/J.ANBEHAV.2005.03.043.

Wright HWY, Gray MM, Wayne RK, Woodroffe RB. 2010. Mating tactics and paternity in a socially monogamous canid, the bat-eared fox (Otocyon megalotis). J. Mammal. 91:437-446. doi: 10.1644/09MAMM-A-046.1.

Wright PC. 1984. Biparental care in Aotus trivirgatus and Callicebus moloch. In: Female primates: studies by women primatologists. Small, MF, editor. Alan R. Liss: New York, NY pp. 59-75.

Wright PC. 2013. Callicebus in Manu National Park: territory, resources, scent marking and vocalizations. In: Evolutionary biology and conservation of titis, sakis and uacaris. Veiga, LM, Barnett, AA, Ferrari, SF, \& Norconk, MA, editors. Cambridge University Press: Cambridge pp. 232-239.

Wright PC. 1990. Patterns of paternal care in primates. Int. J. Primatol. 11:89-102. doi: 10.1007/BF02192783.

Wu JS, Chiang PJ, Lin LK. 2012. Monogamous system in the Taiwan vole Microtus kikuchii inferred from microsatellite DNA and home ranges. Zool. Stud. 51:204-212. doi: Doi 10.1115/1.1518504.

Wuensch KL. 1985. Effects of early paternal presence upon nonhuman offsprings' development. Integr. Comp. Biol. 25:911-923. doi: 10.1093/icb/25.3.911.

Yi Y, Fichtel C, Ham S, Jang H, Choe JC. 2020. Fighting for what it's worth: participation and outcome of inter-group encounters in a pair-living primate, the Javan gibbon (Hylobates moloch). Behav. Ecol. Sociobiol. 74. doi: 10.1007/s00265-020-02879-0.

Zeh JA, Zeh DW. 1996. The evolution of polyandry I: intragenomic conflict and genetic incompatibility. Proc. R. Soc. B Biol. Sci. 263:1711-1717. doi: 10.1098/rspb.1996.0250.

Zeh JA, Zeh DW. 1997. The evolution of polyandry II: post-copulatory defences against genetic incompatibility. Proc. R. Soc. B Biol. Sci. 264:69-75. doi: 10.1098/rspb.1997.0010. 


\section{APPENDIX}

\section{Supplementary information to Chapter 3}

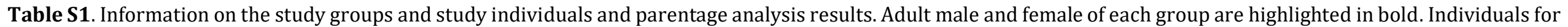
which genetic samples could not be collected are in square brackets. Genotypes of the study animals are not included in here because of the space limitations.

\begin{tabular}{|c|c|c|c|c|c|c|c|c|c|}
\hline $\begin{array}{l}\text { Group } \\
\text { ID }\end{array}$ & $\begin{array}{l}\text { Group } \\
\text { habituated } \\
\text { since }\end{array}$ & $\begin{array}{l}\text { Home } \\
\text { range } \\
\text { size, ha* }\end{array}$ & Animal ID & Sex & Presence during the study period & $\begin{array}{l}\text { Mother } \\
\text { seen } \\
\text { nursing }\end{array}$ & $\begin{array}{l}\text { MtDNA } \\
\text { haplotype }\end{array}$ & $\begin{array}{l}\text { Assigned mother } \\
\text { (Delta score from } \\
\text { Cervus analysis) }\end{array}$ & $\begin{array}{l}\text { Assigned father } \\
\text { (Delta score from } \\
\text { Cervus analysis) }\end{array}$ \\
\hline \multirow[t]{5}{*}{1} & 1997 & 7.81 & Moody & Male & Present from the beginning** & & A & & \\
\hline & & & Pomfrey & Female & Present from the beginning & & $\mathrm{B}$ & & \\
\hline & & & Snape & Male & $\begin{array}{l}\text { Present from the beginning until Sep } \\
2018\end{array}$ & & B & $\begin{array}{l}\text { Pomfrey } \\
(9.61 \mathrm{E}+00)\end{array}$ & Moody $(9.11 \mathrm{E}+00)$ \\
\hline & & & Hermione & Female & $\begin{array}{l}\text { Present from the beginning until } \\
\text { August } 2019\end{array}$ & & $\mathrm{~B}$ & $\begin{array}{l}\text { Pomfrey } \\
(6.60 \mathrm{E}+00)\end{array}$ & Moody $(4.54 \mathrm{E}+00)$ \\
\hline & & & Neville & Male & Born $05 / 06 / 2018^{* * *}$ & Yes & $\mathrm{B}$ & $\begin{array}{l}\text { Pomfrey } \\
(1.23 \mathrm{E}+01)\end{array}$ & Moody $(9.77 \mathrm{E}+00)$ \\
\hline \multirow[t]{3}{*}{2} & Sep-17 & 3.60 & Bill & Male & Present from the beginning & & $\mathrm{C}$ & & \\
\hline & & & Fleur & Female & Present from the beginning & & $\mathrm{D}$ & & \\
\hline & & & Victoire & Male & Born 26/10/2018 & Yes & $\mathrm{D}$ & Fleur $(9.31 \mathrm{E}+00)$ & Bill (1.96E+01) \\
\hline \multirow[t]{5}{*}{3} & Oct-17 & 6.44 & Lucius & Male & Present from the beginning & & E & & \\
\hline & & & Bellatrix & Female & Present from the beginning & & B & & \\
\hline & & & Dolores & Female & $\begin{array}{l}\text { Present from the beginning until Sep } \\
2019\end{array}$ & & $\mathrm{~B}$ & $\begin{array}{l}\text { Bellatrix } \\
(9.05 \mathrm{E}+00)\end{array}$ & Lucius $(7.33 \mathrm{E}+00)$ \\
\hline & & & Sybill & Female & Born Aug 2017 & Yes & B & $\begin{array}{l}\text { Bellatrix } \\
(1.11 \mathrm{E}+01)\end{array}$ & Lucius $(6.17 \mathrm{E}+00)$ \\
\hline & & & [Norris] & Female & Born $11 / 11 / 2018$ & Yes & NA & & \\
\hline \multirow[t]{2}{*}{4} & Jun-18 & 10.10 & Remus & Male & Present from the beginning & & $\mathrm{F}$ & & \\
\hline & & & Rowena & Female & Present from the beginning & & $\mathrm{G}$ & & \\
\hline
\end{tabular}




\begin{tabular}{|c|c|c|c|c|c|c|c|c|c|}
\hline & & & Hedwig & Female & Present from the beginning & & $\mathrm{H}$ & NA & Remus $(1.23 \mathrm{E}+01)$ \\
\hline \multirow[t]{4}{*}{5} & Aug-18 & 4.52 & Hagrid & Male & Present from the beginning & & $\mathrm{B}$ & & \\
\hline & & & Minerva & Female & Present from the beginning & & $\mathrm{I}$ & & \\
\hline & & & Buckbeak & Male & $\begin{array}{l}\text { Present from the beginning until July } \\
2019\end{array}$ & & I & $\begin{array}{l}\text { Minerva } \\
(5.08 \mathrm{E}+00)\end{array}$ & Hagrid $(1.05 \mathrm{E}+01)$ \\
\hline & & & Dobby & Female & Born 20/02/2019 & Yes & $\mathrm{I}$ & $\begin{array}{l}\text { Minerva } \\
(5.46 \mathrm{E}+00)\end{array}$ & Hagrid $(9.68 \mathrm{E}+00)$ \\
\hline \multirow[t]{8}{*}{6} & Aug-17 & 10.09 & Arthur & Male & Present from the beginning & & $\mathrm{B}$ & & \\
\hline & & & Molly & Female & Present from the beginning & & $\mathrm{E}$ & & \\
\hline & & & Gabriel & Male & $\begin{array}{l}\text { Present from the beginning until Sep } \\
2017\end{array}$ & & $\mathrm{E}$ & Molly (1.42E+01) & Arthur $(1.95 \mathrm{E}+00)$ \\
\hline & & & Fred & Male & Present from the beginning & & $\mathrm{E}$ & Molly (1.17E+01) & Arthur $(3.71 \mathrm{E}+00)$ \\
\hline & & & George & Male & Present from the beginning & & $\mathrm{E}$ & Molly $(9.75 \mathrm{E}+00)$ & Arthur $(5.40 \mathrm{E}+00)$ \\
\hline & & & Ron & Male & Born 05/10/2017 & Yes & $\mathrm{E}$ & Molly $(1.03 \mathrm{E}+01)$ & Arthur $(5.13 \mathrm{E}+00)$ \\
\hline & & & Ginny & Female & Born 10/10/2018 & Yes & $\mathrm{E}$ & Molly (3.45E+00) & Arthur $(3.80 \mathrm{E}+00)$ \\
\hline & & & [Rose] & NA & Born Aug 2019 & & $\mathrm{NA}$ & & \\
\hline \multirow[t]{3}{*}{7} & Jul-18 & 7.91 & Rolf & Male & Present from the beginning & & $\mathrm{J}$ & & \\
\hline & & & Luna & Female & Present from the beginning & & $\mathrm{E}$ & & \\
\hline & & & [Lorcan] & NA & $\begin{array}{l}\text { Born Nov 2018, disappeared } \\
\text { summer } 2019\end{array}$ & & NA & & \\
\hline \multirow[t]{3}{*}{8} & Aug-18 & 3.96 & Newt & Male & Present from the beginning & & $\mathrm{E}$ & & \\
\hline & & & Tina & Female & Present from the beginning & & $\mathrm{K}$ & & \\
\hline & & & Harry & Male & Present from the beginning & & $\mathrm{K}$ & Tina $(8.76 \mathrm{E}+00)$ & Newt (1.19E+01) \\
\hline \multirow[t]{4}{*}{9} & Aug-18 & 4.14 & Dean & Male & Present from the beginning & & $\mathrm{K}$ & & \\
\hline & & & [Parvati] & Female & Present from the beginning & & NA & & \\
\hline & & & Cho & Female & Present from the beginning & & $\mathrm{L}$ & NA & Dean $(8.37 \mathrm{E}+00)$ \\
\hline & & & Cedric & Male & Present from the beginning & & $\mathrm{L}$ & NA & Dean $(1.32 \mathrm{E}+01)$ \\
\hline \multirow[t]{2}{*}{10} & Jul-18 & 10.31 & Albus & Male & Present from the beginning & & $\mathrm{M}$ & & \\
\hline & & & Augusta & Female & Present from the beginning & & $\mathrm{N}$ & & \\
\hline
\end{tabular}




\begin{tabular}{|c|c|c|c|c|c|c|c|c|}
\hline & & & Tonx & Male & Present from the beginning & $\mathrm{N}$ & $\begin{array}{l}\text { Augusta } \\
(1.74 \mathrm{E}+01)\end{array}$ & $\begin{array}{l}\text { Albus/Newt } \\
(0.00 \mathrm{E}+00)(\text { see } \\
\text { main text) }\end{array}$ \\
\hline \multirow[t]{2}{*}{11} & Sep-18 & 4.11 & Snape & Male & $\begin{array}{l}\text { Subadult from Group 1, dispersed } \\
\text { and established a new territory with } \\
\text { Narcissa in Sep } 2018 \text { (see main text) }\end{array}$ & $\mathrm{B}$ & $\begin{array}{l}\text { Pomfrey } \\
(9.61 \mathrm{E}+00)\end{array}$ & Moody $(9.11 \mathrm{E}+00)$ \\
\hline & & & Narcissa & Female & $\begin{array}{l}\text { Appeared in the study area shortly } \\
\text { after the dispersal of Snape }\end{array}$ & $\mathrm{F}$ & NA & NA \\
\hline \multirow[t]{4}{*}{12} & Dec-17 & 12.98 & [Binns] & Male & Present from the beginning & NA & & \\
\hline & & & Hooch & Female & Present from the beginning & G & & \\
\hline & & & [Sprout] & NA & Present from the beginning & NA & & \\
\hline & & & [Gilderoy] & NA & Present from the beginning & NA & & \\
\hline \multirow[t]{3}{*}{13} & Jul-18 & 7.62 & [Vernon] & Male & Present from the beginning & NA & & \\
\hline & & & Petunia & Female & Present from the beginning & A & & \\
\hline & & & [Dudley] & NA & Present from the beginning & NA & & \\
\hline \multirow[t]{2}{*}{14} & Jun-18 & NA & $\begin{array}{l}\text { Voldemor } \\
\text { t }\end{array}$ & Male & Present from the beginning & M & & \\
\hline & & & [Tom] & Female & Present from the beginning & NA & & \\
\hline
\end{tabular}

* Estimated using the 95\% fixed kernel density method with ArcGIS Desktop 10.6 (ESRI)

** Data collection started in June 2017 (see the main text)

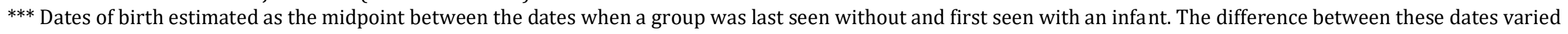
between 0 and 26 days 


\section{Spatial autocorrelation analysis: supplementary results}

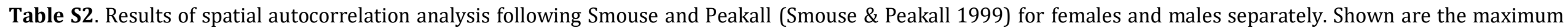

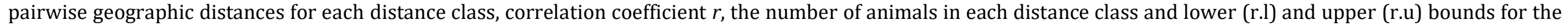
$95 \%$ confidence interval of $r$, obtained by bootstrapping.

\begin{tabular}{|c|c|c|c|c|c|c|c|c|c|}
\hline \multicolumn{5}{|l|}{ Females } & \multicolumn{5}{|l|}{ Males } \\
\hline Distance class, $\mathrm{m}$ & $\mathrm{N}$ animals & $\mathrm{r}$ & r.l & r.u & Distance class, $\mathrm{m}$ & $\mathrm{N}$ animals & $\mathrm{r}$ & r.l & r.u \\
\hline 299.4 & 6 & -0.0830325 & -0.2542848 & 0.21937966 & 292.8 & 4 & -0.2349362 & -0.3567092 & 0.13736895 \\
\hline 598.8 & 18 & -0.0981039 & -0.2324864 & 0.02939302 & 585.6 & 14 & -0.1058502 & -0.2257818 & 0.01909891 \\
\hline 898.2 & 36 & -0.0773378 & -0.1769578 & -0.0002104 & 878.4 & 22 & -0.1169571 & -0.2111225 & 0.01247317 \\
\hline 1197.6 & 26 & -0.0978059 & -0.1831366 & 0.03261151 & 1171.2 & 14 & -0.0903676 & -0.2124641 & 0.00493281 \\
\hline 1497 & 12 & -0.1212871 & -0.240429 & 0.07607147 & 1464 & 12 & -0.068062 & -0.2449801 & 0.0480202 \\
\hline 1796.4 & 10 & -0.063905 & -0.2753533 & 0.08319532 & 1756.8 & 6 & -0.104962 & -0.2914423 & 0.18519218 \\
\hline 2095.8 & 0 & NA & NA & NA & 2049.6 & 4 & -0.029389 & -0.2991212 & 0.24345271 \\
\hline 2395.2 & 8 & -0.1285922 & -0.2708487 & 0.14497517 & 2342.4 & 12 & -0.0757605 & -0.2306265 & 0.04671697 \\
\hline 2694.6 & 6 & -0.1145511 & -0.2889671 & 0.11391038 & 2635.2 & 8 & -0.0743862 & -0.2061112 & 0.07836299 \\
\hline 2994 & 4 & -0.0106682 & -0.3831919 & 0.1271796 & 2928 & 8 & -0.124718 & -0.22446 & 0.08496394 \\
\hline
\end{tabular}
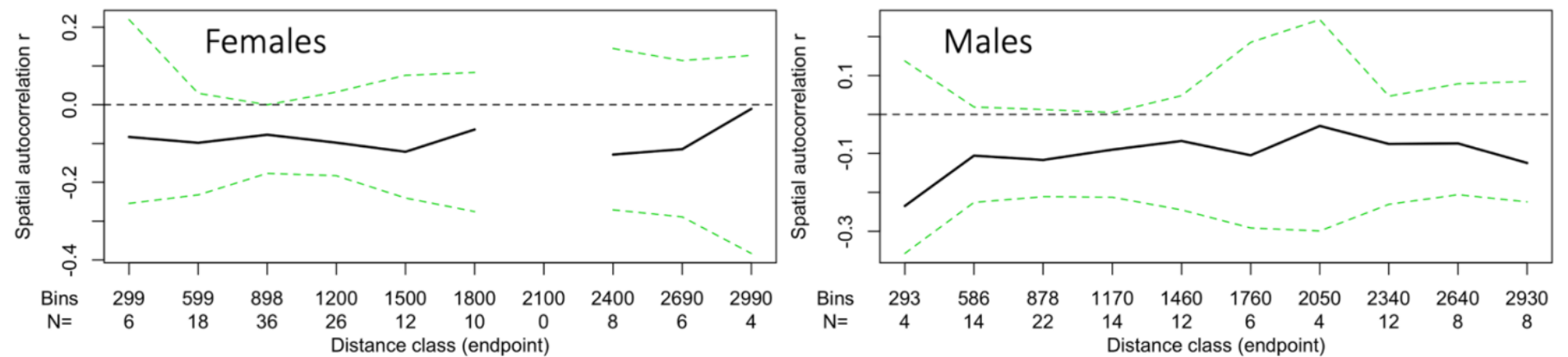

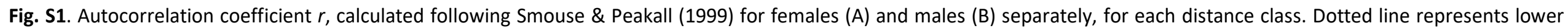
and upper bounds for the $95 \%$ confidence interval of $r$, obtained by bootstrapping. 


\section{Microsatellite genotyping: supplementary methods}

As published microsatellite loci for titi monkeys (Mendoza et al. 2015; Menescal et al. 2009; Martins 2015) revealed unreliable results for our study species, we established a new set of 27 di-repeat microsatellite loci that can be applied not only for our study species but for all New World monkeys (Tables S3 ${ }^{1}$, S4). Therefore, we screened 450 bacterial artificial chromosome (BAC) clones of Plecturocebus moloch (as representative of the Pitheciidae and thus most basal family among New World monkeys) available in Genbank for microsatellites using RepeatMasker (http://www.repeatmasker.org) and extracted orthologous regions (microsatellite plus 1000 bp flanking regions) from the four available New World monkey genomes (Callithrix jacchus calJac3/ASM275486v1, Saimiri boliviensis boliviensis SaiBol1.0, Cebus capucinus imitator Cebus_imitator-1.0, Aotus nancymaae Anan_2.0) available at the UCSC Genome Browser (https://genome.ucsc.edu) and the Ensemble webpage (https://www.ensembl.org/index.html). Only loci with length variation in the microsatellite repeat among the five New World monkey species were selected for further analysis. Further selection criteria were: 1) loci are on different chromosomes according to the Callithrix jacchus genome, 2) primers bind in conserved flanking regions of the microsatellite, 3) primers do not bind in repeat regions such as Alu elements, and 4) primers are close to the microsatellite to minimize amplicon size, thus allowing amplification from low-quality DNA samples. In total 27 loci fulfilled these criteria. Primers were designed to carry adapter nucleotide sequences at their 5' ends (forward primer: 5'-ACACTCTTTCCCTACACGACGCTCT3'; reverse primer: 5'-GTGACTGGAGTTCAGACGTGTGCTCTTCCGATCT-3') to simplify library preparation for genotyping by sequencing on Illumina's MiSeq desktop sequencer.

We then tested if the primers are locus-specific and if these loci can be universally amplified across New World monkeys by conducting singleplex PCRs with each one representative of all New World monkey families and subfamilies (Pitheciidae-Callicebinae: Plecturocebus cupreus, Pitheciidae-Pitheciinae: Chiropotes satanas, Atelidae-Alouattinae: Alouatta seniculus, Atelidae-Atelinae: Ateles fusciceps, Aotidae: Aotus azarae, Cebidae-Cebinae: Sapajus xanthosternos, Cebidae-Saimirinae: Saimiri boliviensis, Callithrichidae: Callimico goeldii). Therefore, we used high-quality DNA extracted from blood or tissue samples available in the Gene Bank of Primates at the German Primate Center. PCR reactions were performed with the Qiagen Multiplex PCR Kit (Qiagen) in a total volume of $25 \mu \mathrm{L}$ and containing $12.5 \mu \mathrm{L} 2 \mathrm{x}$ Multiplex Master Mix, $0.5 \mu \mathrm{L}(0.2 \mu \mathrm{M}$ ) of each primer, $1 \mu \mathrm{L}$ (ca. $20 \mathrm{ng}$ ) of DNA and $10.5 \mu \mathrm{L}$ of RNase-free water. Amplifications were performed with initial denaturation at $95^{\circ} \mathrm{C}$ for 15 min, 40 cycles of denaturation at $94^{\circ} \mathrm{C}$ for $30 \mathrm{sec}$, annealing at $57^{\circ} \mathrm{C}$ for $1.5 \mathrm{~min}$, extension at

\footnotetext{
${ }^{1}$ Genotypes not provided here because of space limitations.
} 
$72^{\circ} \mathrm{C}$ for $1 \mathrm{~min}$ and a final extension at $60^{\circ} \mathrm{C}$ for $30 \mathrm{~min}$. PCR products were checked on $1.5 \%$ agarose gels together with non-template controls and size standards.

Next, we checked if all 27 loci can also be amplified in a single multiplex PCR reaction. Therefore, we again used the eight high-quality DNA extracts and applied the methods (PCR amplification, gel electrophoresis, purification steps, indexing PCR, sequencing on MiSeq platform, data analysis with CHIIMP software) as outlined in the main text. We also checked if the amplification would be more successful when using 3 separate multiplex reactions with the following primer pools: chr01b-chr07a, chr08a-chr12a, chr12b-chrXa (the order is the same as in table S3; reaction details are provided in the main text). The results of these runs, both for the total and 3 separate multiplex reactions, are provided in table S3. Loci chr11f and chr16b failed to amplify in all samples, and loci chr96a and chr06b were amplified in less than half of the samples; the rest of the loci amplified in at least half of the samples. The number of successfully amplified loci varied from 16 (Callimico goeldii and Chiropotes satanas) to 25 (Sapajus xanthosternos, Saimiri boliviensis). Using 3 separate multiplex PCR reactions in most cases yielded more reads than using one total multiplex reaction; the alleles called, however, did not differ between these methods.

\section{Direct observation of dispersal}

In September 2018, we witnessed the dispersal of a subadult male (Snape), the oldest offspring of Group 1, from its natal home range and the formation of a new pair. Group 1 was seen feeding every day during a 2-week period in an Inga edulis tree next to the station buildings. On all occasions, all group members were feeding simultaneously and without any aggression. On September 8, the adult female was aggressively chasing the young adult male while both were leaving the feeding tree. The next day, the group was not seen feeding, and on September 10, the young adult male was feeding alone. On the early morning of September 11, the group was heard calling south-east from the station, and a single individual, presumably the young adult male, was heard from the south-west. On 12 September, the young adult male was encountered in the company of an unknown female south-west of the station. They established in an unoccupied area between the home ranges of Group 1 and Group 6 (Fig. 1 in the main text). Subsequently, this newly formed pair (Group 11) was having intergroup encounters with Group 1 almost every morning; calling and counter-calling were accompanied occasionally by some chasings between the newly formed pair and the adult male from Group 1. Calling and counter-calling ceased by around December 1, 2018. As of September 2019, Group 11 was still occupying the same home range and had an infant born in February 2019. 


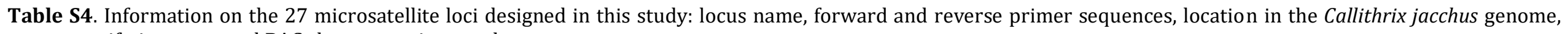
repeat motif, size range and $\mathrm{BAC}$ clone accession number.

\begin{tabular}{|c|c|c|c|c|c|c|}
\hline Locus & Forward primer & Reverse primer & Location CalJac3 & $\begin{array}{l}\text { Repeat } \\
\text { motif }\end{array}$ & $\begin{array}{l}\text { Size } \\
\text { range, bp }\end{array}$ & $\begin{array}{l}\text { PMOL BAC } \\
\text { clone Acc. } \\
\text { Nr. }\end{array}$ \\
\hline PMOL_chr01b & TGCCAAGGGCTTTCTGA & AAACAYCTCTTTTGTAGAAG & chr01:195360092-195360177; 1q & TG & $75-105$ & AC187426.3 \\
\hline PMOL_chr02a & AGGCTGTGTTTGTGGTG & TTCTTGCACCTTTCTCAAT & chr02:47304050-47304166; 2p & TG & $117-123$ & AC188274.1 \\
\hline PMOL_chr02b & GGTCAAACCAGGGCAAA & TTATTTGCAATTTATAGCCTA & chr02:72990959-72991145; 2q & GC, AC & $139-187$ & AC186467.2 \\
\hline PMOL_chr03e & GTAAGATGGGAGATTAGC & ATTACAGCCCTATGGTAG & chr03:167338029-167338166; 3q & TG & $112-138$ & AC188271.1 \\
\hline PMOL_chr04a & AAGAAAAGTGAGATCCCC & TGTTGAGGTTGCCCAGA & chr04:42663591-42663697; 4q & TG & $97-131$ & AC187951.2 \\
\hline PMOL_chr05c & GCCCCACACCTGCTTT & GACCACCTGCCACATG & chr05:9622769-9622890; 5p & TG & $102-145$ & AC200393.3 \\
\hline PMOL_chr05g & TTTTGAATCCTTTCCAGTG & ATTCCTGAGCTCAGGTTT & chr05:1808047-1808162; 5p & $\mathrm{CA}$ & $112-122$ & AC187427.2 \\
\hline PMOL_chr06a & ATGGAACAGCCAATGAGA & TYTTAAGTAGAGGAGTGAC & chr06:91748983-91749061; 6q & CT & $78-104$ & AC209150.1 \\
\hline PMOL_chr06b & AGCTGTGAACATTTTGTAC & AATTACAGCATATTCATGCT & chr06:12239728-12239857; 6p & CA, CT & $120-142$ & AC157438.1 \\
\hline PMOL_chr07a & TGCCTGAGAACTGCACA & GACATGCTTTCCСTCAAT & chr07:60957721-60957833; 7q & TG, CG & $106-134$ & AC188276.1 \\
\hline PMOL_chr08a & GTAGAGCTAAGAGGCTC & TCATTTAAGAATAGGCAATG & chr08:84171068-84171190; 8q & TG, GC & $87-123$ & AC203507.2 \\
\hline PMOL_chr08f & GGTAGTTGTTGGCACTG & ACATGATATATAAGGGGAG & chr08:119173148-119173258; 8q & AC, GT, GA & $91-123$ & AC174857.2 \\
\hline PMOL_chr09a & GTTCTGCCTTAAGGTTTC & CATAAARATCCACTTTAAAAC & chr09:10544128-10544250; 9p & TG & $111-125$ & AC244997.1 \\
\hline PMOL_chr10a & GTGCAGGGACAAATCTG & TGGCCTTGTAAATAAAATGT & chr10:125088776-125088873; 10q & $\mathrm{CA}$ & $90-110$ & AC188357.2 \\
\hline PMOL_chr10b & AGAAGCCATGTCAATTAAG & ATTGTCAAAATATGGCTCC & chr10:20581481-20581580; 10q & TG & $86-114$ & AC186463.2 \\
\hline PMOL_chr11e & CCTGGGCTTACAGAACC & TACCTATCTAGCTCATTTC & chr11:14130663-14130767; 11p & $\mathrm{CA}$ & $105-122$ & AC186115.2 \\
\hline PMOL_chr11f & TATGCAATATATTTCAAATATC & GATGCTGATGCATTTGTG & chr11:68860458-68860555; 11q & $\mathrm{CA}$ & $92-112$ & AC172721.2 \\
\hline PMOL_chr12a & AGTGACTGTTTAACCACC & TCCATTTTACAACTGCTGA & chr12:24867917-24868037; 12p & TG & $107-147$ & AC193735.2 \\
\hline PMOL_chr12b & CAAATATAGACTCATTAAATG & CTACAGGTATGTTCCTTG & chr12:70126419-70126506; 12q & $\mathrm{AC}$ & $88-110$ & AC190366.1 \\
\hline PMOL_chr13a & GCTCAGACAGGATGATG & AGAGCTTTGGACTCAGG & chr13:101919673-101919801; 13q & $\mathrm{CA}$ & $95-129$ & AC187952.2 \\
\hline PMOL_chr13b & GCAAGAGTGGTCTGGC & CAAGAATTATCTATGCAGG & chr13:6811774-6811922; 13p & $\mathrm{CA}$ & $131-161$ & AC151890.2 \\
\hline PMOL_chr14b & TTAGGCATTGATATAAGGC & CAGAAAAATTTCATTGCCC & chr14:10607938-10608090; 14 & CA, TA, GA & $143-189$ & AC207510.1 \\
\hline PMOL_chr16a & AGCACATGACTGGCCTT & AAGATAACAAATAGAATTGGA & chr16:76914251-76914383; 16 & $\mathrm{CA}$ & $117-133$ & AC190368.2 \\
\hline
\end{tabular}




\begin{tabular}{|c|c|c|c|c|c|c|}
\hline PMOL_chr16b & CTGCAGACTAGCCTCAT & GGATTTACAAAGGAAATAGA & chr16_GL285730_r:61519-61625; 16 & TG, AG & $107-133$ & AC186936.2 \\
\hline PMOL_chr18a & AGCTGGTTTGGGAGATAA & TGCTCAGATYCTCAGTCT & chr18:5739039-5739139; 18 & $\mathrm{AC}$ & $70-101$ & AC189181.2 \\
\hline PMOL_chr21a & GAATTTCTTCAGTTCAACTA & CRGTGTTAAGATTGAAAATG & chr21:37574331-37574478; 21q & GA, TG & $130-148$ & AC174416.2 \\
\hline PMOL_chrXa & ATGTGTTGTGGACCTAAG & TCCAAGAAGTAATCGTGTA & chrX:125242408-125242500; Xq & $\mathrm{AC}$ & $93-117$ & AC237129.1 \\
\hline
\end{tabular}

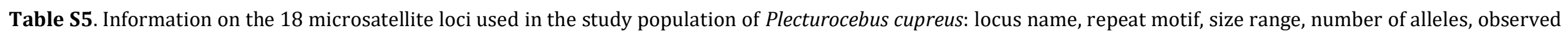

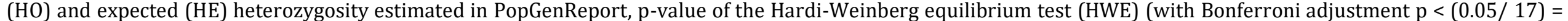

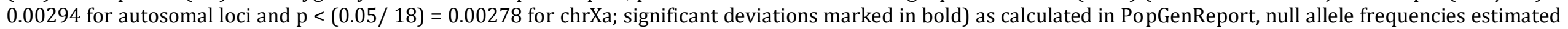
using Brookfield1 method in MicroChecker and number of individuals typed for each locus.

\begin{tabular}{|c|c|c|c|c|c|c|c|c|c|}
\hline Locus & FullName & $\begin{array}{l}\text { Repeat } \\
\text { motif }\end{array}$ & Size range, bp & $\begin{array}{l}\text { Number of } \\
\text { alleles }\end{array}$ & HO & HE & HWE-P & Null F & $\mathrm{N}$ ind typed \\
\hline 1 & chr01b & $\mathrm{TG}$ & 89-123 & 12 & 0.609 & 0.905 & 0.000 & 0.072 & 28 \\
\hline 4 & chr03e & $\mathrm{AC}$ & 106-138 & 12 & 0.826 & 0.889 & 0.020 & -0.013 & 39 \\
\hline 6 & chr05c & $\mathrm{AC}$ & $103-125$ & 9 & 0.739 & 0.742 & 0.730 & -0.022 & 40 \\
\hline 7 & chr05g & $\mathrm{TG}$ & $102-116$ & 8 & 0.913 & 0.828 & 0.747 & -0.056 & 41 \\
\hline 8 & chr06a & GA & 90-119 & 12 & 0.870 & 0.857 & 0.525 & -0.046 & 41 \\
\hline 10 & chr07a & $\mathrm{CA}$ & $106-122$ & 8 & 0.913 & 0.805 & 0.119 & -0.048 & 39 \\
\hline 11 & chr08a & $\mathrm{AC}$ & $86-93$ & 6 & 0.826 & 0.757 & 0.162 & -0.072 & 40 \\
\hline 12 & chr08f & $\mathrm{AC}$ & $109-143$ & 11 & 0.913 & 0.874 & 0.039 & 0.039 & 36 \\
\hline 13 & chr09a & CA & $107-129$ & 8 & 0.870 & 0.782 & 0.173 & -0.050 & 41 \\
\hline 14 & chr10a & GT & $94-114$ & 9 & 0.783 & 0.840 & 0.742 & 0.059 & 41 \\
\hline 16 & chr11e & GT & $90-124$ & 9 & 0.696 & 0.775 & 0.514 & 0.060 & 41 \\
\hline 19 & chr12b & $\mathrm{TG}$ & 74-90 & 3 & 0.565 & 0.466 & 0.835 & -0.002 & 38 \\
\hline 20 & chr13a & GT & $101-106$ & 4 & 0.348 & 0.502 & 0.099 & -0.001 & 41 \\
\hline 22 & chr14b & $\mathrm{TG}$ & $147-173$ & 14 & 0.826 & 0.897 & 0.078 & 0.001 & 39 \\
\hline 23 & chr16a & GT & $113-119$ & 4 & 0.174 & 0.309 & 0.011 & 0.027 & 39 \\
\hline 25 & chr18a & GT & $78-124$ & 16 & 0.826 & 0.912 & 0.034 & -0.007 & 38 \\
\hline 26 & chr21a & GA & $157-161$ & 5 & 0.696 & 0.716 & 0.000 & -0.117 & 34 \\
\hline 27 & chrXa & $\mathrm{TG}$ & $105-127$ & 10 & 0.872 & 1.000 & $0.816^{*}$ & -0.068 & 19 \\
\hline
\end{tabular}




\section{Supplementary information to Chapter 4}

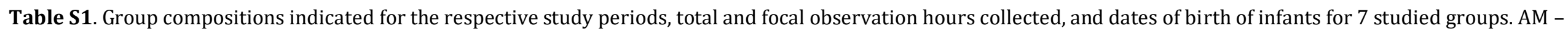

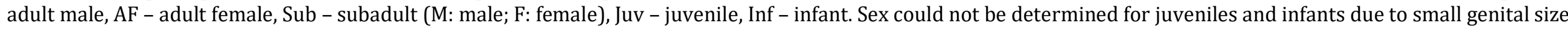

\begin{tabular}{|c|c|c|c|c|c|c|c|c|c|}
\hline Group & Study period & $\begin{array}{l}\text { Total sampling } \\
\text { time, h }\end{array}$ & Focal sampling time, $h$ & $\mathbf{A M}$ & $\mathbf{A F}$ & Sub & Juv & Inf & Infant date of birth* \\
\hline \multirow[t]{2}{*}{1} & Jun - Dec 2017 & \multirow[t]{2}{*}{482.4} & \multirow[t]{2}{*}{139.4} & 1 & 1 & $1(\mathrm{M})$ & 1 & 0 & - \\
\hline & Sep - Dec 2018 & & & 1 & 1 & $1(\mathrm{M})$ & 1 & 1 & 05.06 .2018 \\
\hline \multirow[t]{2}{*}{2} & Sep - Oct 2017 & \multirow[t]{2}{*}{479.9} & \multirow[t]{2}{*}{55.3} & 1 & 1 & 0 & 0 & 0 & - \\
\hline & $\begin{array}{l}\text { Jul 2018, Oct - Dec } \\
2018\end{array}$ & & & 1 & 1 & 0 & 0 & 1 & 26.10 .2018 \\
\hline \multirow[t]{2}{*}{3} & Oct - Dec 2017 & \multirow[t]{2}{*}{393.6} & \multirow[t]{2}{*}{20.8} & 1 & 1 & 0 & 1 & 1 & 09.2018 \\
\hline & Jun 2018 & & & 1 & 1 & $1(\mathrm{M})$ & 1 & 0 & - \\
\hline 4 & $\begin{array}{l}\text { Jun - Jul 2018, Sep - } \\
\text { Oct } 2018\end{array}$ & 387.2 & 86.0 & 1 & 1 & 0 & 1 & 0 & - \\
\hline 5 & Aug - Oct 2018 & 294.8 & 38.3 & 1 & 1 & 0 & 1 & 0 & - \\
\hline \multirow[t]{2}{*}{6} & Aug - Oct 2017 & \multirow[t]{2}{*}{520.3} & \multirow[t]{2}{*}{38.4} & 1 & 1 & $1(\mathrm{M})-2(\mathrm{M}, \mathrm{F})$ & 1 & 1 & 05.10 .2017 \\
\hline & Jul 2018 & & & 1 & 1 & $2(\mathrm{M}, \mathrm{F})$ & 1 & 0 & - \\
\hline 7 & Aug 2018, Nov 2018 & 192.6 & 12.7 & 1 & 1 & 0 & 0 & 1 & 01.11 .2018 \\
\hline
\end{tabular}

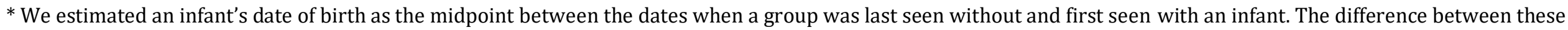

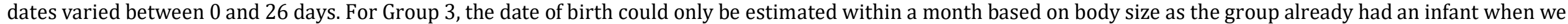

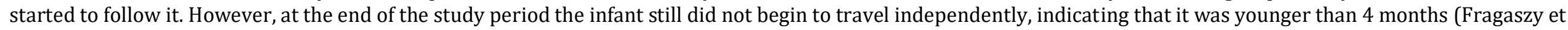
al. 1982; Wright 1984). 
Table S2. Results of the models on (1) rates of proximity and affiliation; (2) grooming reciprocity between pair mates. Indicated are estimates, standard errors, confidence intervals, results of likelihood ratio tests, and the range of estimates obtained when dropping levels of random effects one at a time.

\begin{tabular}{|l|l|l|l|l|l|l|l|l|l|}
\hline Term & Estimate & SE & $\begin{array}{l}\text { Lower } \\
\text { CI }\end{array}$ & $\begin{array}{l}\text { Upper } \\
\text { CI }\end{array}$ & $\chi 2$ & df & $\begin{array}{l}\text { P- } \\
\text { value }\end{array}$ & min & max \\
\hline
\end{tabular}

\section{Model 1: rates of proximity and affiliation}

The model was fairly stable; maximum Variance Inflation Factor: 1.517; dispersion parameter $=0.996$

\begin{tabular}{|l|l|l|l|l|l|l|l|l|l|}
\hline Intercept & -0.378 & 0.123 & -0.618 & -0.138 & - & - & & -0.484 & -0.230 \\
\hline $\begin{array}{l}\text { Infant } \\
\text { presence (1) }\end{array}$ & -1.019 & 0.234 & -1.495 & -0.543 & 16.524 & 1 & 0.000 & -1.102 & -0.801 \\
\hline $\begin{array}{l}\text { Group size } \\
\text { (2) }\end{array}$ & -0.325 & 0.201 & -0.720 & 0.069 & 3.759 & 1 & 0.053 & -0.410 & -0.142 \\
\hline Rainfall (3) & 0.074 & 0.138 & -0.197 & 0.345 & 0.266 & 1 & 0.610 & 0.075 & 0.252 \\
\hline
\end{tabular}

\section{Model 2: grooming reciprocity}

The model was stable; maximum Variance Inflation Factor: 1.783; dispersion parameter $=0.789$

\begin{tabular}{|l|l|l|l|l|l|l|l|l|l|}
\hline Intercept & 0.128 & 0.132 & -0.130 & 0.387 & - & - & - & 0.054 & 0.193 \\
\hline $\begin{array}{l}\text { Infant } \\
\text { presence (1) }\end{array}$ & 1.348 & 0.352 & 0.657 & 2.039 & 15.403 & 1 & 0.000 & 1.309 & 1.457 \\
\hline $\begin{array}{l}\text { Group size } \\
\text { (2) }\end{array}$ & -0.038 & 0.123 & -0.278 & 0.203 & 0.094 & 1 & 0.759 & -0.212 & 0.037 \\
\hline Rainfall (3) & -0.329 & 0.152 & -0.627 & -0.032 & 3.919 & 1 & 0.048 & -0.406 & -0.201 \\
\hline
\end{tabular}

(1) Dummy coded with absence of infant being the reference category

(2) Z-transformed; mean \pm sd of the original value: $3.216 \pm 0.877$

(3) Z-transformed; mean \pm sd of the original value: $91.754 \pm 79.121$ 
Table S3. Time and participation in the observed intergroup encounters. Individual participation could only be determined for the groups followed by the observers. $\mathrm{AM}$ - adult male, AF - adult female, SM - subadult male, SF - subadult female, Juv - juvenile. Shaded rows indicate the encounters initiated by males.

\begin{tabular}{|c|c|c|c|c|c|c|c|c|c|}
\hline Date & Time & $\begin{array}{l}\text { Group } \\
\text { followed/encounter } \\
\text { with group (3) }\end{array}$ & $\begin{array}{l}\text { Participants } \\
\text { (from the group } \\
\text { followed) }\end{array}$ & Initiator & $\begin{array}{c}\text { AM } \\
\text { calling }\end{array}$ & $\begin{array}{c}\text { AM } \\
\text { chasing }\end{array}$ & $\begin{array}{c}\text { AF } \\
\text { calling }\end{array}$ & $\begin{array}{c}\text { AF } \\
\text { chasing }\end{array}$ & Notes \\
\hline 09.09 .17 & $8: 26-9: 00$ & $6 / 2$ & $\mathrm{AM}+\mathrm{SM}$ & AM & yes & yes & no & no & AF and Juv did not participate \\
\hline 13.09 .17 & $\begin{array}{l}\text { 06:02- } \\
6: 17\end{array}$ & $2 / 2$ & $\mathrm{AM}+\mathrm{AF}$ & $\mathrm{AM}$ & yes & yes & yes & no & $\begin{array}{l}\text { AM was the first to start and the } \\
\text { last to stop calling }\end{array}$ \\
\hline 13.09 .17 & $\begin{array}{l}10: 22- \\
10: 36 \\
\end{array}$ & $2 / 6$ & $\mathrm{AM}+\mathrm{AF}$ & $-(1)$ & yes & yes & yes & no & \\
\hline 16.09 .17 & $\begin{array}{l}11: 10- \\
11: 22\end{array}$ & $2 / 6$ & $\mathrm{AM}+\mathrm{AF}$ & - & yes & yes & yes & no & \\
\hline 21.06 .18 & $\begin{array}{l}08: 46- \\
8: 50\end{array}$ & $6 / 3$ & $\begin{array}{l}\mathrm{AM}+\mathrm{AF}+\mathrm{SM}+ \\
\mathrm{SF}\end{array}$ & AM & yes & yes & yes & no & $\begin{array}{l}\text { AF and SF joined for calling only in } \\
\text { the end of the encounter but did not } \\
\text { participate in chasing }\end{array}$ \\
\hline 17.07.18 & $\begin{array}{l}10: 22- \\
10: 46\end{array}$ & $6 / 2$ & $\begin{array}{l}\mathrm{AM}+\mathrm{AF}+\mathrm{SM}+ \\
\mathrm{SF}+\mathrm{Juv}\end{array}$ & AM & yes & yes & yes & yes & $\begin{array}{l}\text { AM was the first to start and the } \\
\text { last to stop calling }\end{array}$ \\
\hline 19.07.18 & $8: 50-8: 53$ & $2 / 3$ & $\mathrm{AM}+\mathrm{AF}$ & - & yes & yes & yes & no & \\
\hline 25.07 .18 & $7: 48-7: 50$ & $3 / 2$ & $\mathrm{AM}+\mathrm{AF}+\mathrm{SM}$ & - & yes & yes & yes & no & \\
\hline 16.08 .18 & $6: 05-6: 11$ & 7/unhabituated group & $\mathrm{AM}+\mathrm{AF}$ & - & yes & $\mathrm{N} / \mathrm{A}^{(4)}$ & yes & $\mathrm{N} / \mathrm{A}$ & \\
\hline 17.08 .18 & $7: 00-7: 02$ & 7/unhabituated group & $\mathrm{AM}+\mathrm{AF}$ & - & yes & $\mathrm{N} / \mathrm{A}$ & yes & $\mathrm{N} / \mathrm{A}$ & \\
\hline 18.09 .18 & $5: 38-5: 55$ & $1 / 11$ & $\mathrm{AM}+\mathrm{AF}+\mathrm{Juv}$ & - & yes & $\mathrm{N} / \mathrm{A}$ & yes & $\mathrm{N} / \mathrm{A}$ & \\
\hline 18.09 .18 & $6: 31-6: 46$ & $1 / 11$ & $\mathrm{AM}+\mathrm{AF}$ & - & yes & $\mathrm{N} / \mathrm{A}$ & yes & $\mathrm{N} / \mathrm{A}$ & \\
\hline 18.09 .18 & $7: 31-7: 58$ & $1 / 11$ & $\mathrm{AM}+\mathrm{AF}+\mathrm{Juv}$ & - & yes & $\mathrm{N} / \mathrm{A}$ & yes & $\mathrm{N} / \mathrm{A}$ & $\begin{array}{l}\text { Juv called only for the last } 10 \mathrm{~min} \text { of } \\
\text { the encounter }\end{array}$ \\
\hline 19.09 .18 & $6: 33-7: 15$ & $1 / 11$ & $\mathrm{AM}+\mathrm{AF}$ & $\mathrm{AM}$ & yes & yes & yes & no & \\
\hline 12.10 .18 & $\begin{array}{l}10: 31- \\
11: 15\end{array}$ & $1 / 11$ & $A M+A F$ & AM & yes & yes & yes & no & $\begin{array}{l}\text { AM called and chased SM from Group } \\
11 ; A F \text { from Group } 1 \text { and AF from } \\
\text { Group } 11 \text { only called but did not } \\
\text { chased }\end{array}$ \\
\hline 13.10 .18 & $8: 52-9: 10$ & $1 / 11$ & $A M+A F$ & - & yes & yes & yes & yes & \\
\hline 15.10 .18 & $6: 43-7: 41$ & $1 / 11$ & $A M+A F+J u v$ & - & yes & yes & yes & no & \\
\hline
\end{tabular}




\begin{tabular}{|c|c|c|c|c|c|c|c|c|c|}
\hline 15.10 .18 & 8:59-9:07 & $1 / 11$ & $A M+A F$ & - & yes & yes & yes & no & \\
\hline 30.11 .18 & $6: 35-6: 38$ & $1 / 11$ & AM & AM & yes & yes & no & no & $\begin{array}{l}\text { AM called and chased SM from Group } \\
11 ; \text { neither AF from Group } 1 \text { nor AF } \\
\text { from Group } 11 \text { called or chased }\end{array}$ \\
\hline 07.12 .18 & 9:56-10:16 & $2 / 3$ & $A M+A F$ & AM & yes & yes & yes & no & $\begin{array}{l}\text { AM was the first to start and the last } \\
\text { to stop calling; AF only called and did } \\
\text { not chase }\end{array}$ \\
\hline 11.12 .18 & $6: 17-6: 22$ & $2 / 3$ & $\mathrm{AM}+\mathrm{AF}$ & AM & yes & yes & yes & no & $\begin{array}{l}\text { AM was the first to start and the last } \\
\text { to stop calling }\end{array}$ \\
\hline
\end{tabular}

(1) - indicates an encounter that did not have any clear initiator

(2) On 11 September 2018, the subadult male dispersed from Group 1 and by 18 September established a new territory with an unknown (to the observers) female next to the home range of his natal group. Since then and until 7 December 2018, when we stopped following Group 1, we observed 6 intergroup encounters between Group 1 and the newly established pair (indicated here as Group 11)

(3) We never observed Groups 4 and 5 engaging in intergroup encounters. Most likely, it is due to the absence of territory overlap with neighbouring groups; this notion is indirectly supported by the fact that we never observed any intergroup encounters in Group 1 until September 2018 (see (2))

(4) Chasing data could not be collected because of the poor observation conditions 


\section{Supplementary information to Chapter 5}

Table A1. Mean daily proportions of time allocated to different activities and to arthropods in the diet for adult females and males of all study groups. "-" indicates no data available for the respective period.

\begin{tabular}{|c|c|c|c|c|c|}
\hline \multirow[t]{2}{*}{ Activity } & \multirow[t]{2}{*}{ Group } & \multicolumn{2}{|c|}{ Females } & \multicolumn{2}{|c|}{ Males } \\
\hline & & Without infant & With infant & Without infant & With infant \\
\hline \multirow[t]{7}{*}{ Feeding } & 1 & 0.172 & 0.267 & 0.168 & 0.049 \\
\hline & 2 & 0.155 & 0.246 & 0.095 & 0.063 \\
\hline & 3 & 0.365 & 0.424 & 0.513 & 0.355 \\
\hline & 4 & 0.229 & - & 0.185 & - \\
\hline & 5 & 0.203 & - & 0.111 & - \\
\hline & 6 & 0.172 & 0.767 & 0.297 & 0.076 \\
\hline & 7 & 0.104 & 0.615 & 0.070 & 0.236 \\
\hline \multirow[t]{7}{*}{ Resting } & 1 & 0.459 & 0.600 & 0.492 & 0.855 \\
\hline & 2 & 0.357 & 0.542 & 0.447 & 0.822 \\
\hline & 3 & 0.378 & 0.360 & 0.378 & 0.457 \\
\hline & 4 & 0.478 & - & 0.528 & - \\
\hline & 5 & 0.648 & - & 0.757 & - \\
\hline & 6 & 0.548 & 0.178 & 0.505 & 0.443 \\
\hline & 7 & 0.752 & 0.177 & 0.317 & 0.710 \\
\hline \multirow[t]{7}{*}{ Social } & 1 & 0.232 & 0.004 & 0.266 & 0.024 \\
\hline & 2 & 0.363 & 0.062 & 0.341 & 0.052 \\
\hline & 3 & 0.139 & 0.067 & 0.038 & 0.060 \\
\hline & 4 & 0.206 & - & 0.211 & - \\
\hline & 5 & 0.049 & - & 0.068 & - \\
\hline & 6 & 0.098 & 0.000 & 0.119 & 0.425 \\
\hline & 7 & 0.000 & 0.136 & 0.595 & 0.018 \\
\hline \multirow[t]{7}{*}{ Moving } & 1 & 0.137 & 0.128 & 0.114 & 0.073 \\
\hline & 2 & 0.126 & 0.151 & 0.117 & 0.063 \\
\hline & 3 & 0.136 & 0.132 & 0.071 & 0.129 \\
\hline & 4 & 0.086 & - & 0.077 & - \\
\hline & 5 & 0.099 & - & 0.063 & - \\
\hline & 6 & 0.182 & 0.056 & 0.169 & 0.056 \\
\hline & 7 & 0.143 & 0.071 & 0.018 & 0.036 \\
\hline \multirow{7}{*}{$\begin{array}{l}\text { Feeding on } \\
\text { arthropods }\end{array}$} & 1 & 0.160 & 0.341 & 0.325 & 0.328 \\
\hline & 2 & 0.276 & 0.701 & 0.296 & 0.353 \\
\hline & 3 & 0.152 & 0.331 & 0.048 & 0.005 \\
\hline & 4 & 0.535 & - & 0.427 & - \\
\hline & 5 & 0.515 & - & 0.522 & - \\
\hline & 6 & 0.351 & 1.000 & 0.086 & 0.153 \\
\hline & 7 & 1.000 & 0.251 & 0.438 & 0.087 \\
\hline
\end{tabular}


Table A2. Results of the models of the daily proportion of feeding time in females for (1) infant dependency period (19 weeks after birth); (2) period of high intensity lactation (28 weeks after birth). Indicated are estimates and standard errors, confidence intervals, results of likelihood ratio tests, and the range of estimates obtained when dropping levels of random effects one at a time.

\begin{tabular}{|l|l|l|l|l|l|l|l|l|l|}
\hline Term & Estimate & SE & $\begin{array}{l}\text { Lower } \\
\text { CI }\end{array}$ & $\begin{array}{l}\text { Upper } \\
\text { CI }\end{array}$ & $\chi 2$ & df & P-value & min & max \\
\hline
\end{tabular}

\section{Model 1: feeding time in females during infant dependency period}

dispersion parameter $=0.92 ; 234$ daily proportion values from 7 females

\begin{tabular}{|l|l|l|l|l|l|l|l|l|l|}
\hline Intercept & -0.457 & 0.100 & -0.654 & -0.261 & - & - & - & -0.553 & -0.375 \\
\hline Infant presence (1) & 0.537 & 0.212 & 0.122 & 0.952 & 6.610 & 1 & 0.010 & 0.337 & 0.768 \\
\hline Group size (2) & -0.176 & 0.192 & -0.552 & 0.201 & 1.094 & 1 & 0.296 & -0.272 & -0.104 \\
\hline Rainfall (3) & -0.053 & 0.098 & -0.245 & 0.138 & 0.267 & 1 & 0.606 & -0.186 & 0.009 \\
\hline
\end{tabular}

Model 2: feeding time in females during period of high intensity lactation

dispersion parameter $=0.93 ; 234$ daily proportion values from 7 females

\begin{tabular}{|l|l|l|l|l|l|l|l|l|l|}
\hline Intercept & 0.464 & 0.101 & -0.661 & -0.267 & - & - & - & -0.562 & -0.364 \\
\hline Infant presence (1) & 0.372 & 0.196 & -0.012 & 0.755 & 3.729 & 1 & 0.053 & 0.171 & 0.586 \\
\hline Group size (2) & -0.236 & 0.230 & -0.688 & 0.216 & 1.240 & 1 & 0.265 & -0.382 & -0.092 \\
\hline Rainfall (3) & -0.097 & 0.090 & -0.274 & 0.080 & 0.970 & 1 & 0.324 & -0.231 & -0.038 \\
\hline
\end{tabular}

(1) Dummy coded with absence of infant being the reference category

(2) Z-transformed to a mean of 0 and a standard deviation of 1 ; mean and sd of the original value were 3.216 and 0.877 , respectively

(3) Z-transformed to a mean of 0 and a standard deviation of 1 ; mean and sd of the original value were 91.754 and 79.121, respectively 
Table A3. Results of the models of the daily proportion of feeding time in lactating females as a function of daily proportion of (1) resting time; (2) social time; (3) moving time. Indicated are estimates and standard errors, confidence intervals, results of likelihood ratio tests, and the range of estimates obtained when dropping levels of random effects one at a time.

\begin{tabular}{|c|c|c|c|c|c|c|c|c|c|}
\hline Term & Estimate & SE & $\begin{array}{l}\text { Lower } \\
\text { CI }\end{array}$ & $\begin{array}{l}\text { Upper } \\
\text { CI }\end{array}$ & $\chi^{2}$ & $\mathrm{df}$ & P-value & $\min$ & $\max$ \\
\hline \multicolumn{9}{|c|}{ dispersion parameter $=1.05 ; 57$ daily proportion values from 5 females } & \\
\hline Intercept & -0.206 & 0.110 & -0.421 & 0.009 & - & - & - & -0.293 & -0.110 \\
\hline Feeding time $(1)$ & -0.856 & 0.113 & -1.077 & -0.064 & 42.328 & 1 & 0.000 & -0.936 & -0.664 \\
\hline \multicolumn{10}{|c|}{$\begin{array}{l}\text { Model 2: feeding time as a function of social time } \\
\text { dispersion parameter }=1.22 ; 57 \text { daily proportion values from } 5 \text { females }\end{array}$} \\
\hline Intercept & -1.265 & 0.128 & -1.517 & -1.013 & - & - & - & -1.362 & -1.187 \\
\hline Feeding time (1) & -0.347 & 0.127 & -0.596 & -0.098 & 7.480 & 1 & 0.006 & -0.416 & -0.325 \\
\hline \multicolumn{10}{|c|}{$\begin{array}{l}\text { Model 3: feeding time as a function of moving time } \\
\text { dispersion parameter }=1.12 ; 57 \text { daily proportion values from } 5 \text { females }\end{array}$} \\
\hline Intercept & -0.640 & 0.077 & -0.791 & -0.489 & - & - & - & -0.696 & -0.609 \\
\hline Feeding time $(1)$ & -0.188 & 0.077 & -0.338 & -0.037 & 5.799 & 1 & 0.016 & -0.326 & -0.146 \\
\hline
\end{tabular}

(1) Z-transformed to a mean of 0 and a standard deviation of 1 ; mean and sd of the original value were 0.342 and 0.301 , respectively 


\section{DECLARATION}

I hereby declare that I have written this thesis independently and with no other aids or sources than quoted.

Sofya Dolotovskaya, Göttingen, 07.09.20 